\title{
Zum Einfluss baulicher Randbedingungen auf das dynamische Verhalten von WIB-Eisenbahnbrücken
}

\author{
Von der Fakultät für Bauingenieurwesen \\ der Rheinisch-Westfälischen Technischen Hochschule Aachen \\ zur Erlangung des akademischen Grades eines Doktors der Ingenieurwissenschaften \\ genehmigte Dissertation
}

vorgelegt von

Tim Rauert

Berichter: Universitätsprofessor Dr.-Ing. Markus Feldmann

Universitätsprofessor Dr.-Ing. Dr.h.c. Gerhard Sedlacek

Universitätsprofessor Dr.-Ing. habil. Carsten Könke

Tag der mündlichen Prüfung: 01. Juli 2011

Diese Dissertation ist auf den Internetseiten der Hochschulbibliothek online verfügbar. 


\section{Kurzfassung}

Die Weiterentwicklung des europäischen Eisenbahnnetzes steht unter dem Vorzeichen des vermehrten Einsatzes von Hochgeschwindigkeitszügen und einer signifikanten Anhebung der Betriebsgeschwindigkeiten. Um die Auswirkungen von dynamischen Effekten aus Verkehrslasten auf Eisenbahnbrücken im Rahmen einer Bemessung hinreichend genau vorhersagen zu können, ist die Kenntnis der wesentlichen dynamischen Systemeigenschaften notwendig. Nur so können bemessungsrelevante Resonanzeffekte, die bei hohen Geschwindigkeiten grundsätzlich nicht auszuschließen sind, rechnerisch auf zuverlässige Weise erfasst werden.

Messungen insbesondere an WIB-Überbauten zeigen jedoch, dass eine realistische Abschätzung von Brückeneigenfrequenzen schwierig ist. Tatsächliche und rechnerische Werte weichen in vielen Fällen erheblich voneinander ab. Ursache hierfür ist unter anderem die Nichtberücksichtigung baulicher Randbedingungen, da diese teilweise erheblichen Einfluss auf die Steifigkeits- und Dämpfungseigenschaften des Systems haben.

Im Rahmen der vorliegenden Arbeit wurden zunächst Messungen an WIBÜberbauten durchgeführt und ausgewertet, um das Spektrum konstruktiver Ausführungsformen von tragendem Überbau, Lagerung sowie der herkömmlich als nicht tragend angesehenen Aufbauten zu erfassen und deren Einfluss auf Eigenfrequenz und Dämpfung weiter abzuklären. Die bisherigen o. g. Erfahrungen konnten dabei bestätigt werden.

Darauf aufbauend wurden Finite-Elemente-Berechnungen zur Simulation von Zugüberfahrten im Hochgeschwindigkeitsbereich durchgeführt - mit dem Ziel, die Wirkung der als wesentlich erkannten konstruktiven Besonderheiten qualitativ und quantitativ zu beurteilen.

Um dabei den Effekt der Schotterkopplung mechanisch abbilden zu können, der sich bei kontinuierlichen Schotterschichten auf baulich voneinander getrennten Einzelüberbauten einstellt, wurden Experimente an Eisenbahnschotter zur Herleitung eines vereinfachten Koppelmodells vorgenommen.

Im Rahmen der numerischen Untersuchungen wurde festgestellt, dass sich insbesondere Steifigkeitsbeiträge von Randkappen und Schutzschicht erheblich auf die Brückendynamik auswirken, wobei dieser Effekt verstärkt bei Brücken mit kleinen Stützweiten auftritt. Weiterhin wurde festgestellt, dass sich die steifigkeitsbeeinflussende Wirkung der unterschiedlichen baulichen Randbedingungen prinzipiell ähnlich abbilden lässt, indem die globale Biegesteifigkeit des Brückenquerschnitts ersatzweise vergrößert wird. 
Die Auswertung der Bauwerksmessungen sowie die Ergebnisse der experimentellen und numerischen Untersuchungen münden in Vorschlägen, um die dynamische Bemessung von WIB-Brücken zu verbessern. Unter anderem wurde eine Bemessungshilfe erarbeitet, mit deren Hilfe sich die effektive Steifigkeit von WIBQuerschnitten näherungsweise bestimmen lässt. Das Verfahren basiert auf Korrekturbeiwerten zur Modifikation rechnerischer Querschnittssteifigkeiten. Unter Verwendung der Bemessungshilfe lässt sich eine zuverlässigere Prognose des dynamischen Systemverhaltens von WIB-Überbauten ableiten. Wie im Rahmen eines Berechnungsbeispiels gezeigt wird, kann das vorgeschlagene Konzept in einfacher Weise in die aktuelle Bemessungspraxis integriert werden. 


\section{Abstract}

Enhancements of the European railway system are amongst other things mainly characterized by increased usage of high speed trains and significantly raised operating speeds. To predict effects of train loading on railway bridges for a dynamic design adequately, knowledge of the system's significant dynamic characteristics is required. Only this ensures that resonance effects, which generally cannot be screened out for high speeds, can be covered analytically by reliable values.

Measurements especially carried out on filler beam bridges have shown though that a realistic estimation of eigenfrequencies of bridges is difficult. Actual values and measured ones differ significantly in many cases. In particular nonconsideration of structural constraints is seen as reason for this, given that they have an immense effect on a bridge's stiffness and damping characteristics.

Within the scope of this thesis, firstly measurement campaigns on various filler beam bridges were conducted and analysed, in order to study the range of constructional detailing with regard to span lengths, cross-section design, bearings and additional non supporting structural elements on the bridge deck, furthermore in order to investigate the influence of these structural constraints on the bridge's dynamic behaviour. The above mentioned experiences were confirmed by the obtained measurement results.

Based on this, finite element calculations were carried out to simulate train passages with high velocities on filler beam bridges. These numerical studies aimed at both qualitative and quantitative assessment of dynamic effects resulting from the most influential constraints.

For being able to consider interaction effects numerically, caused by continuous ballast layers on separated bridge decks, experimental tests on railway ballast were conducted. The outcome of these tests is a simplified mechanical model that can be applied in numerical calculations for taking into account the load transfer between ballast-connected structures.

The numerical investigations revealed that stiffness contributions of non supporting concrete elements, attached to the cross section, have significant influence on the bridge dynamics, particularly in case of small span lengths. Furthermore it was ascertained that all effects on the system rigidity, resulting from the various structural constraints, can be considered in a similar way within numerical analysis - by increasing the cross section's global bending stiffness. 
The evaluation of the measurement campaigns as well as the results from the experimental and numerical investigations lead to proposals for an improved dynamic bridge design. A design tool was prepared in order to determine the effective bending stiffness of filler beam decks approximately. It is based on correction factors to modify calculated stiffness values. By using this design tool, the prediction of the dynamic behaviour of filler beam bridges becomes more reliable. The procedure can easily be included within the current design practise which is finally shown by means of an example of dynamic bridge design. 


\section{Inhaltsverzeichnis}

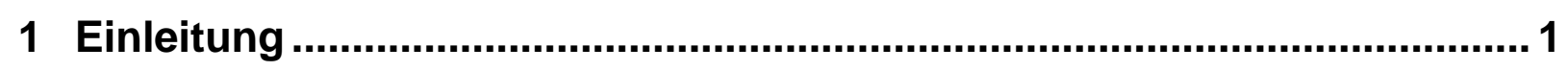

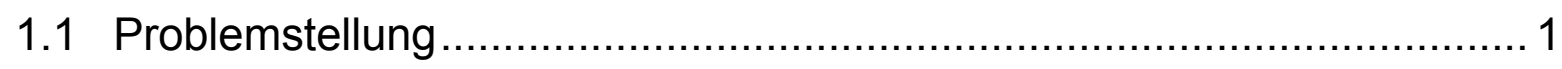

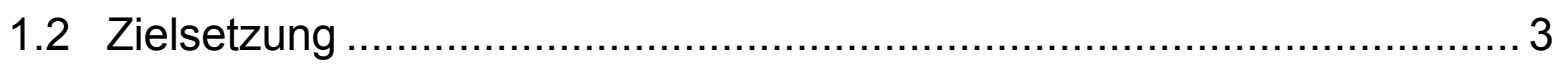

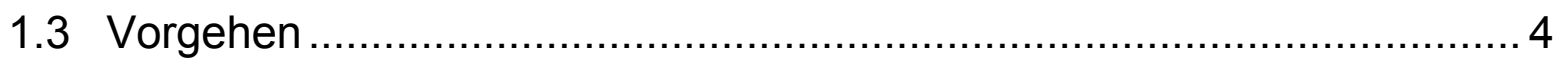

2 Stand der Technik und Grundlagen ...................................................... 7

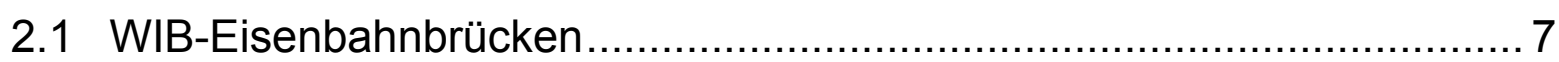

2.2 Dynamik und dynamische Bemessung von Eisenbahnbrücken für den Hochgeschwindigkeitsverkehr .................................................. 9

2.3 Auswirkungen baulicher Randbedingungen auf die Brückendynamik .. 16

2.4 Zeitschrittintegrationsrechungen zur Simulation von Zugüberfahrten ... 19

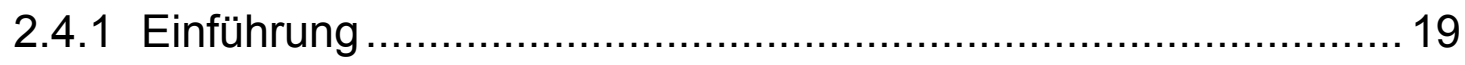

2.4.2 Direkte Integrationsverfahren ............................................. 20

2.4.3 Zeitschrittintegrationsrechnungen mit InfoCAD........................2 23

2.5 Zum Dämpfungsbeitrag von Eisenbahnschotter ............................... 27

2.5.1 Viskose Dämpfung ............................................................ 27

2.5.2 Hysteretische Dämpfung ................................................... 29

2.5.3 Berücksichtigung nichtlinearer Effekte .................................... 31

2.5.4 Äquivalente viskose Dämpfung........................................... 32

3 Messungen an WIB-Eisenbahnbrücken .................................................... 34

3.1 Messkampagnen der Deutschen Bahn ........................................... 34

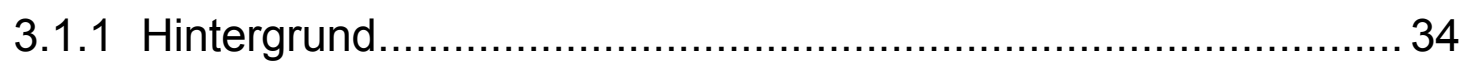

3.1.2 Auswertung der Messungen ............................................... 34

3.2 Bauwerks-Monitoring an der WIB-Brücke EÜ Erfttalstraße..................39

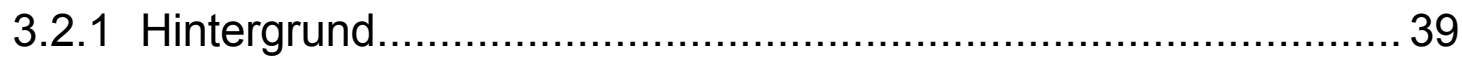

3.2.2 Bauliche Durchbildung und Messsystem ................................. 40 
3.2.3 Allgemeines zur Messauswertung …....................................44

3.2.4 Zugtypen und Überfahrtgeschwindigkeiten ..............................45

3.2.5 Berechnung der Brückendurchbiegungen ...............................48

3.2.6 Maximalwerte von Beschleunigungen und Durchbiegungen.......52

3.2.7 Eigenfrequenzen und Dämpfung ..........................................55

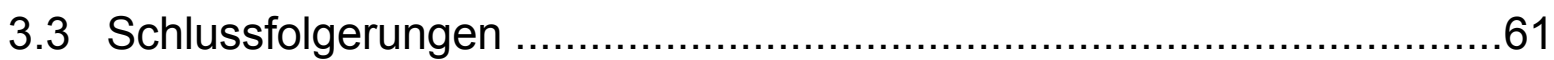

4 Experimentelle Untersuchungen an Eisenbahnschotter .......................63

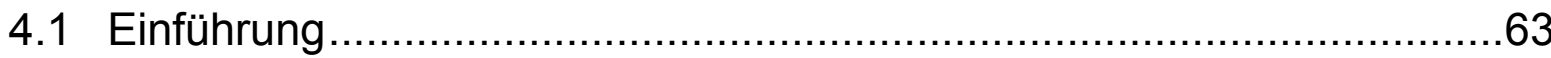

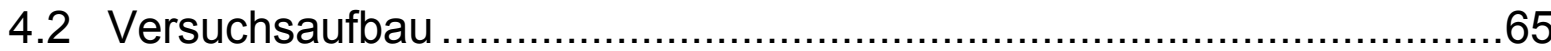

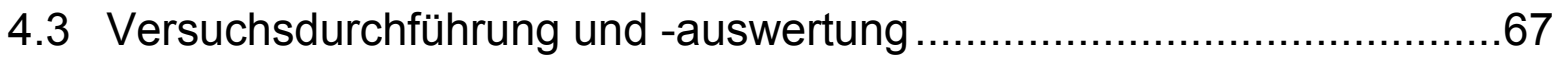

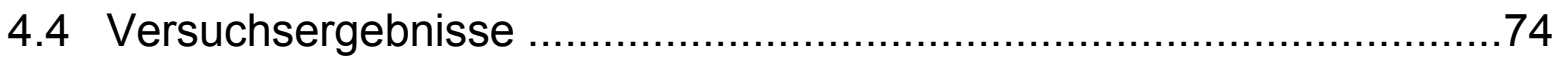

4.5 Verwendung der experimentellen Ergebnisse in numerischen Berechnungen.

\section{Numerische Untersuchungen an WIB-Brücken zum Einfluss}

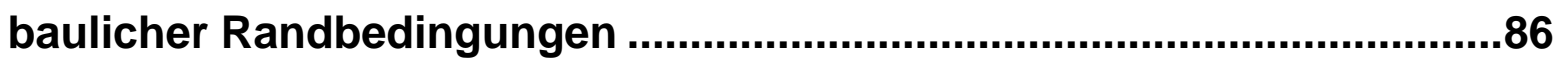

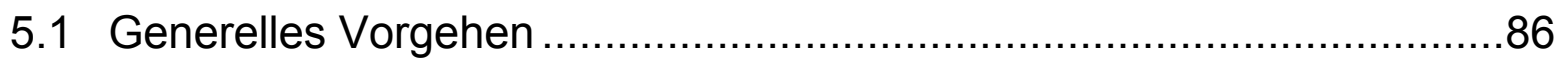

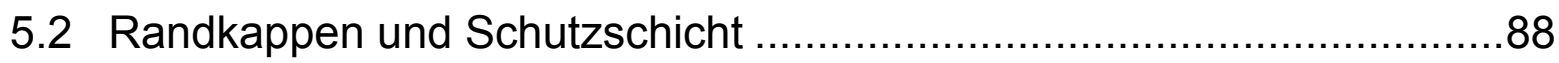

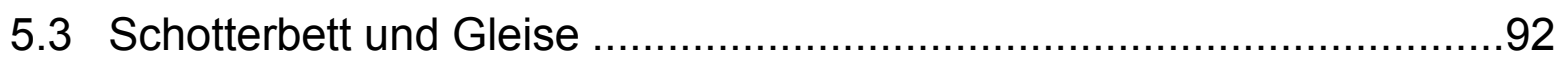

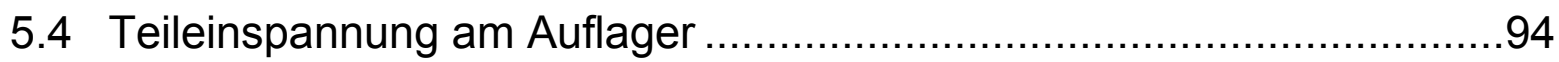

5.5 Kopplung von nebeneinander liegenden WIB-Überbauten durch

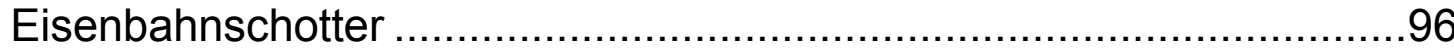

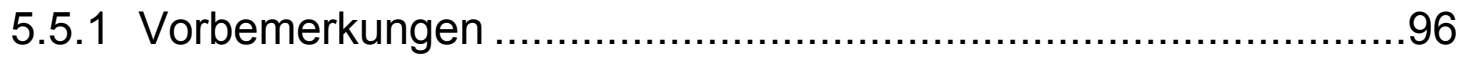

5.5.2 Voruntersuchungen zum Einfluss von Koppelfedern in

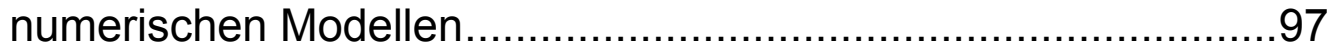

5.5.3 Untersuchung der gemessenen Überbauten..........................104

6 Schlussfolgerungen und Vorschläge für die Bemessung....................109

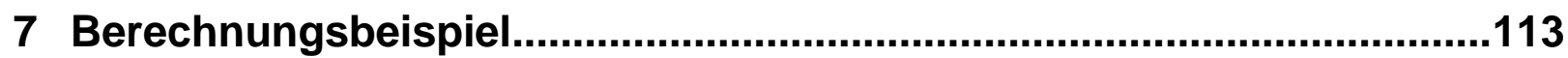

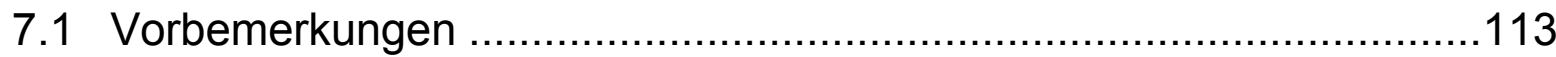




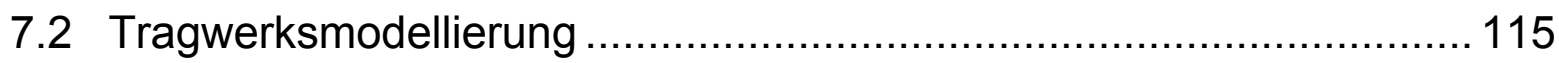

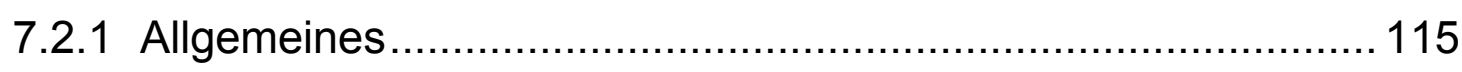

7.2.2 Modell 1: Nackter WIB-Querschnitt..................................... 115

7.2.3 Modell 2: Steifigkeitsmodifikation mit Korrekturfaktor .............. 119

7.2.4 Modell 3: Zusätzliche Berücksichtigung von Koppelfedern....... 120

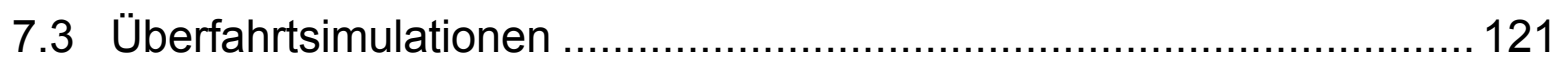

7.4 Ergebnisse und Vergleich mit Messwerten .................................... 123

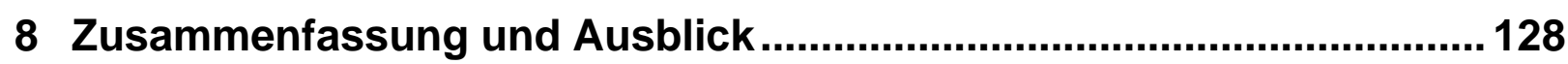

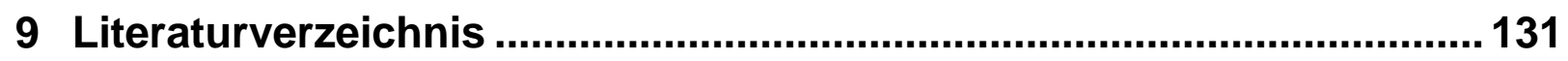




\section{Einleitung}

\subsection{Problemstellung}

Bis etwa zum Jahr 2000 wurden Eisenbahnbrücken im Netz der Deutschen Bahn ausschließlich unter Ansatz statischer Lasten bemessen, wobei dynamische Einflüsse, die nicht aus Resonanz resultieren, mit Hilfe von dynamischen Beiwerten am statischen Lastansatz Berücksichtigung fanden, siehe z. B. [2]. Der Hochgeschwindigkeitsverkehr stellt jedoch eine besondere Beanspruchung von Schienenwegen und insbesondere von Brücken dar. So wurden beispielsweise in Frankreich nach Einführung des TGV Schäden im befahrenen Schienennetz festgestellt. Verschiebungen des Gleisrostes, Risse und Abplatzungen an Brückenbauwerken aus Beton sowie Schwellen-Hohllagen traten auf [2] [69] [70]. Auch im Deutschen Schienennetz wurden auf Brücken neuer Hochgeschwindigkeitsstrecken Gleislageverschlechterungen und ein starker Abrieb von Schottersteinen (sogenannte „weiße Stellen“) festgestellt [15] [29] [68].

Die Schäden an den Brücken wurden hauptsächlich darauf zurückgeführt, dass der Einsatz von Hochgeschwindigkeitszügen mit konstanten Achsabständen zu einer Resonanzanregung von Systemen führte, deren Eigenfrequenzen sich in ungünstigen Bereichen befanden [2]. Auch wenn diese Resonanzbeanspruchung nicht in jedem Fall unmittelbare Schäden nach sich ziehen muss, so kann sie dennoch einerseits eine erhöhte Ermüdungsbeanspruchung von Brücken und andererseits beschleunigten Schotterabrieb und damit verbunden häufige Instandhaltungsmaßnahmen bedeuten oder auch eine Einschränkung des Fahrkomforts für Passagiere zur Folge haben. Bereits im Entwurfstadium sollte mit dieser Problematik sensibel umgegangen werden [44].

Dies gab Anlass für genauere dynamische Untersuchungen, die von der Deutschen Bahn durchgeführt wurden, wobei nicht nur Neubauten, sondern auch Bestandsbauten Gegenstand waren. Stellte sich heraus, dass ein Brückenbauwerk aufgrund seiner rechnerischen dynamischen Eigenschaften anfällig für Resonanzeffekte war, so wurden Schwingungsmessungen an der Brücke durchgeführt. Dabei wurden u. a. die tatsächlichen Werte von Eigenfrequenzen und effektive Dämpfungswerte bestimmt, um anschließend genauere und zuverlässigere dynamische Berechnungen durchführen zu können [6].

Gegenstand dieser Untersuchungen waren insbesondere WIB-Brücken, die im Hochgeschwindigkeitsnetz der Deutschen Bahn aufgrund ihrer einfachen und schnellen Bauweise häufig vertreten sind. Denn mit typischen Spannweiten von 
10 bis $25 \mathrm{~m}$ ergaben sich in vielen Fällen rechnerische Eigenfrequenzen, die im Hinblick auf Resonanzeffekte als kritisch eingeschätzt wurden. Im Rahmen eines in Kooperation mit der Deutschen Bahn durchgeführten Forschungsprojektes [52] wurde dem Lehrstuhl für Stahlbau und Leichtmetallbau der RWTH Aachen eine Vielzahl der so entstandenen Messberichte mit zugehörigen Ergebnisprotokollen zur Verfügung gestellt, die im Rahmen dieser Arbeit weiter ausgewertet werden konnten. Es zeigte sich, dass die im Vorfeld rechnerisch ermittelten bzw. gemäß Bemessungsvorgabe angesetzten dynamischen Eigenschaften signifikant von den tatsächlichen gemessenen Werten abweichen. Die erste Eigenfrequenz wird rechnerisch um mindestens $25 \%$ unterschätzt, die Systemdämpfung ist in Wirklichkeit höher, als nach Norm anzusetzen, siehe hierzu [46]. Weitere Beispiele dafür, dass rechnerisch ermittelte Eigenfrequenzen von kurzen und mittelweit spannenden Brücken häufig deutlich niedriger sind als die tatsächlich vorhandenen Werte sind z.B. in [33] [43] [50] [53] [57] [61] zu finden.

Die Unterschätzung der Eigenfrequenz hat die Bestimmung zu niedriger kritischer Geschwindigkeiten zur Folge, bei denen die Brücke bei Überfahrten von Ganzzügen in Resonanz angeregt wird. Eine niedrigere Systemdämpfung führt im Rahmen des dynamischen Nachweises zur Überschätzung der nachzuweisenden Maximalwerte von Beschleunigungen, Verformungen, etc. Die dynamischen Brückenparameter werden also von rechnerischer Seite generell konservativ abgeschätzt. Damit sind auch die rechnerisch ermittelten dynamischen Bauwerksreaktionen ungünstiger als das tatsächliche Verhalten. Für die Fehleinschätzung von Eigenfrequenz und Dämpfung werden verschiedene Effekte, die derzeit nicht berücksichtigt werden, als ursächlich angesehen, vgl. z.B. [2]:

- Steifigkeitsbeiträge von Ober- und Ausbauelementen

- Lagerreibung

- Kopplung von nebeneinander liegenden Überbauten infolge kontinuierlicher Schotterschichten

- Erhöhte Dämpfungswerte durch Reibvorgänge im Schotter

- Dämpfungsbeiträge von Oberbau- und Ausrüstungselementen

Mit den bisherigen Ansätzen liegt die Bemessung zwar auf der sicheren Seite, jedoch hat dies zur Folge, dass bestimmte Brückenbauwerke auf Basis rechnerischer Untersuchungen nicht für den Hochgeschwindigkeitsverkehr bzw. für bestimmte Zugtypen des Hochgeschwindigkeitsverkehrs freigegeben werden können, was einen erheblichen Nachteil für die effiziente Nutzung des Schienennetzes bedeutet. Als Konsequenz daraus sind häufig Messungen erforder- 
lich um nachzuweisen, dass als zunächst kritisch angesehene Brückenbauwerke entgegen den rechnerischen Prognosen mit deutlich höheren Geschwindigkeiten befahren werden dürfen [6]. Sind Messungen nicht möglich, wird häufig mit kostenintensiven Verstärkungsmaßnahmen auf die rechnerische Resonanzanfälligkeit reagiert, oder die maximal zulässigen Geschwindigkeiten werden in Abhängigkeit vom Zugtyp auf Basis theoretischer Überlegungen begrenzt. In Österreich sind Bauwerksmessungen an resonanzgefährdeten Eisenbahnbrücken sogar explizit vorgeschrieben [39], siehe auch [23].

\subsection{Zielsetzung}

Eine bessere Vorhersage des dynamischen Systemverhaltens von Brücken kurzer bis mittlerer Spannweiten, insbesondere von WIB-Brücken ist dringend erforderlich. Die Voraussetzung für eine zuverlässige Vorhersage ist eine möglichst exakte Bestimmung der dynamischen Eigenschaften des Brückenbauwerks (Steifigkeit, Massenverteilung, Eigenfrequenzen, Dämpfung). Nur wenn diese Eingangsgrößen im Vorfeld der dynamischen Nachweise hinreichend genau abgeschätzt werden können, lassen sich anschließend zuverlässige Prognosen ableiten.

Im Rahmen der Arbeit soll zunächst das dynamische Verhalten von WIBEisenbahnbrücken unter Berücksichtigung der besonderen konstruktiven Ausbildung des tragenden Überbaus, der Lagerung und der herkömmlich als nicht tragend angesehenen Aufbauten geklärt werden. Denn insbesondere bei Zugüberfahrt ist die dynamische Antwort des Brückenbauwerks nur relativ grob bekannt, wie im Verlauf der Arbeit noch weiter deutlich wird.

Darauf aufbauend sollen Modelle zur Abbildung des dynamischen Verhaltens bei Zugüberfahrten entwickelt werden, mit denen man in der Lage ist, wirklichkeitsnahe Überfahrtsimulationen im Hochgeschwindigkeitsbereich durchzuführen.

Schließlich soll eine praxistaugliche Bemessungshilfe erarbeitet werden, mit der in Kenntnis der baulichen Randbedingungen eine zuverlässigere Prognose des dynamischen Verhaltens von WIB-Brücken ermöglicht wird.

Dabei sind diese konstruktiven Randbedingungen bei bestehenden Bauwerken in der Regel bekannt. Bei Neubauten können sie in der Entwurfsphase sogar bewusst vorgegeben werden. Der Nutzen einer solchen Bemessungshilfe besteht darin, dass kostspielige und zeitintensive Messungen nach Fertigstellung eines Brückenbauwerks vermieden werden können. Der planende Ingenieur kann bereits in der Entwurfsphase zuverlässige Prognosen hinsichtlich des dy- 
namischen Systemverhaltens treffen. Bei der Umnutzung bestehender Brückenbauwerke für den Hochgeschwindigkeitsverkehr ist eine zuverlässige Abschätzung der Brückendynamik Voraussetzung, um bauwerksgerechte Geschwindigkeitsvorgaben zu machen, ohne auf Messungen am Bauwerk angewiesen zu sein.

\subsection{Vorgehen}

Nach der Einleitung werden in Abschnitt 2 der Stand der Technik und die Grundlagen dargestellt. Darin wird die WIB-Bauweise erläutert, insbesondere auf charakteristische konstruktive Merkmale und auf dynamische Eigenschaften eingehend. Anschließend wird das Vorgehen bei der dynamischen Bemessung von Eisenbahnbrücken für den Hochgeschwindigkeitsverkehr beschrieben. Hierbei wird besonderes Augenmerk auf die normative Behandlung des dynamisch ungünstigen Falles der Resonanzanregung durch Ganzzüge gerichtet. Die bereits erwähnte Problematik, die sich bei ungenauer Bestimmung der bemessungsrelevanten Eingangsparameter Eigenfrequenz und Dämpfung ergibt, wird in diesem Zusammenhang erläutert. Weiterhin wird der in der Literatur (siehe [32] [37] [65]) als „cancellation phenomenon“ bezeichnete Effekt erklärt, der später bei der Auswertung von Messdaten relevant ist. Nachfolgend wird beschrieben, wie sich bauliche Randbedingungen auf das dynamische Verhalten von WIB-Brücken auswirken und welche Folgen dies für die dynamische Bemessung hat. Das Grundlagenkapitel fortsetzend, wird gezeigt, wie sich mit Hilfe direkter Integrationsverfahren Bewegungsdifferentialgleichungssysteme lösen lassen, die sich bei der Berechnung dynamischer Systeme nach der Finiten Elemente Methode ergeben. Dabei wird speziell auf das NewmarkVerfahren eingegangen, das im Rahmen der Arbeit bei der Simulation von Zugüberfahrten zur Anwendung kam. Abschließend wird die Frage nach dem Dämpfungsbeitrag von Eisenbahnschotter bei der dynamischen Anregung von Brückenbauwerken mit Schotteroberbau erörtert.

In Abschnitt 3 beschäftigt sich die Arbeit mit Messungen an WIB-Brücken, bei denen jeweils die dynamischen Parameter Eigenfrequenz und Dämpfung bestimmt wurden. Neben diesen Messkampagnen wurde zur genaueren Untersuchung der dynamischen Eigenschaften von WIB-Brücken im Rahmen eines Europäischen Forschungsprojektes ein eigens entwickeltes Monitoringsystem an der Brücke EÜ Erfttalstraße auf der HGV-Strecke Köln-Düren installiert, um sowohl Dehnungen als auch Beschleunigungen während Zugüberfahrten messtechnisch zu erfassen. Aus den Messdaten lassen sich u. a. Brückenverformungen während Zugüberfahrten ableiten, die im späteren Verlauf der Arbeit zur 
Validierung von numerischen Modellen verwendet wurden. Von Interesse sind auch die klimatischen Einflüsse, die im Zuge der Auswertung von Messdaten festgestellt werden konnten. Die Ergebnisse der behandelten Messkampagnen werden im Detail dargestellt und in den Kontext der Arbeit gestellt. Ferner werden Erläuterungen zum Begriff und Phänomen der Dämpfung gegeben.

WIB-Überbauten des Deutschen Eisenbahnnetzes sind in der Regel mit Schotteroberbau ausgerüstet. Besteht die Brücke aus zwei oder mehr eingleisigen baulich voneinander getrennten Überbauten, so kommt der durchgehenden Schotterschicht im Hinblick auf das dynamische Systemverhalten eine besondere Bedeutung zu. Diese bauliche Randbedingung führt zu einer Kopplung der Überbauten während der Beanspruchung durch Zugverkehr, so dass neben den belasteten auch die unbelasteten Brückenträger dynamisch angeregt werden. Bisher gibt es keine experimentell hergeleiteten Ansätze zur numerischen Abbildung dieses Effekts.

Es wurden deshalb experimentelle Untersuchungen an Eisenbahnschotter durchgeführt, die in Abschnitt 4 behandelt werden. Mit Hilfe der Versuche wurden Parameter bereitgestellt, um den durch den Schotter verursachten Koppeleffekt unter Verwendung eines ingenieurmäßigen Modells mechanisch zu beschreiben. Auf Grundlage der experimentellen Ergebnisse wird ausführlich diskutiert, wie die abgeleiteten Parameter in numerischen Berechnungen verwendet werden können, um die Kopplung benachbarter WIB-Überbauten realistisch abzubilden.

Anschließend wurden, ausgehend von den Resultaten der Messkampagnen und der experimentellen Untersuchungen sowie den sich daraus ergebenden Schlussfolgerungen, ergänzende numerische Berechnungen durchgeführt, um die Einflüsse baulicher Randbedingungen auf das dynamische Verhalten von WIB-Brücken genauer abzuklären. Dabei wurden unter anderem Zugüberfahrten mit dem Programm InfoCAD simuliert. Eine Verifizierung der Berechnungsergebnisse erfolgt durch Vergleich mit Messdaten, die aus den in Abschnitt 3 behandelten Bauwerksmessungen stammen. Die numerischen Untersuchungen werden in Abschnitt 5 behandelt.

Die Auswertungen der Bauwerksmessungen sowie die Ergebnisse der experimentellen und numerischen Untersuchungen bilden die Grundlage für Vorschläge zur Verbesserung der dynamischen Bemessung von WIB-Brücken. In Abschnitt 6 wird unter anderem eine Bemessungshilfe vorgestellt, die in Abstimmung mit der Deutschen Bahn erarbeitet wurde und mit deren Hilfe sich die effektive Steifigkeit von WIB-Querschnitten näherungsweise bestimmen lässt. 
Mit ihr lässt sich eine zuverlässigere Prognose des dynamischen Systemverhaltens von WIB-Überbauten ableiten. Dabei wird ein Konzept mit Korrekturbeiwerten verfolgt, das mit der derzeitigen Bemessungspraxis vereinbar ist und in einfacher Weise in die aktuelle Normung integriert werden kann.

Um die Anwendbarkeit und Zuverlässigkeit des Verfahrens zu zeigen, wird in Abschnitt 7 im Rahmen eines Berechnungsbeispiels vorgeführt, wie sich die Bemessungsvorschläge praktisch umsetzen lassen. Zu diesem Zweck wurde die Erfttalbrücke ausgewählt, die wie oben erwähnt mit einem Monitoringsystem ausgerüstet wurde. Daher ergab sich hier die Möglichkeit, die numerischen Ergebnisse mit Messwerten aus dem Bauwerks-Monitoring zu vergleichen. Die Zuverlässigkeit des Verfahrens konnte somit direkt überprüft werden.

Schließlich wird die Arbeit in Abschnitt 8 zusammengefasst und mit einem Ausblick abgeschlossen, in dem $u$. a. die Frage erörtert wird, inwieweit sich das Vorgehen auch auf andere Überbauformen übertragen lässt, um so das Anwendungsgebiet des vorgeschlagenen Bemessungskonzeptes mit Korrekturbeiwerten zu erweitern. 


\section{Stand der Technik und Grundlagen}

\subsection{WIB-Eisenbahnbrücken}

Ein Viertel aller Brücken im deutschen Eisenbahnnetz sind als WIB-Überbauten (Walzträger in Beton) ausgeführt [55] (Bild 2.1). Diese Bauweise wurde erstmalig bereits Ende des 19. Jahrhunderts angewandt, bis sie dann Anfang des 20. Jahrhunderts bis in die 30er Jahre eine Blütezeit erlebt hat. Doch auch heutzutage stellt die WIB-Bauweise aus Gründen der Einfachheit und aufgrund ihrer geringen Bauhöhen eine wirtschaftliche und robuste Konstruktionsform dar, die bei Neubauten regelmäßig zur Anwendung kommt. Eine detaillierte Darstellung der gegenwärtig gängigen Verbundbauweisen im Eisenbahnbrückenbau (zu denen auch die WIB-Bauweise zu zählen ist) kann beispielsweise [54] entnommen werden.

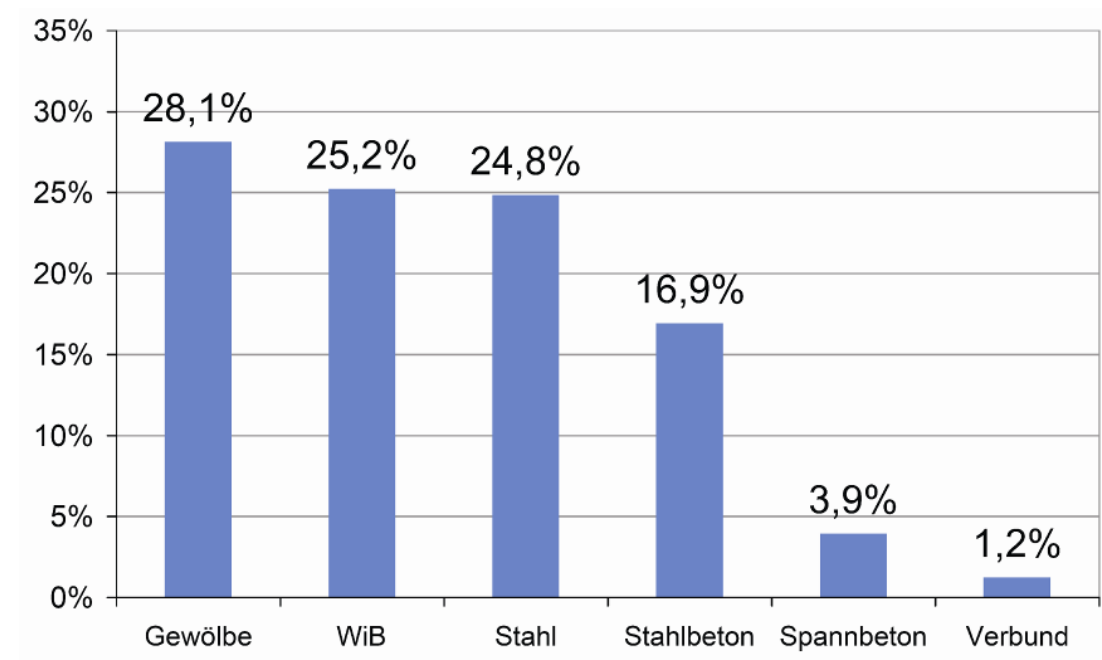

Bild 2.1: Verteilung der Bauarten im Brückenbestand des deutschen Bahnnetzes (Prozentwerte bezogen auf die Gesamtanzahl) [55]

WIB-Brücken (Beispiele in Bild 2.2) werden bevorzugt als Einfeldträger mit Schotteroberbau und Spannweiten von $10 \mathrm{~m}$ bis $20 \mathrm{~m}$ gebaut, wobei Stützweiten bis zu 30 m möglich sind. Unter dem Gesichtspunkt der Tragfähigkeit sind bei Verwendung der Baustoffe C 30/37 und S 355 Schlankheiten L/h von 25 bis 30 herstellbar. Damit lassen sich im Vergleich zu anderen Bauweisen deutlich geringere Bauhöhen realisieren, was bei Neubauten in beengten Verhältnissen von großem Vorteil sein kann. Auch für den Ersatzneubau erweisen sich die hohen Schlankheiten als vorteilhaft. In den meisten Fällen werden bei Ersatzneubauten die geforderten lichten Höhen aufgrund erhöhter Anforderungen vergrößert. Eine Anhebung der Gleisgradiente kommt aus Kostengründen in der Regel nicht in Betracht, so dass sich für den Ersatzneubau geringere Bauhöhen 
als im Bestand ergeben, die bei Verwendung von WIB-Überbauten eingehalten werden können. Für Durchlauftragwerke ist die WIB-Bauweise weniger geeignet, da die Vollstöße der Walzträger mit großem konstruktiven Aufwand verbunden und hinsichtlich ihrer Dauerhaftigkeit anfällig sind.

a)

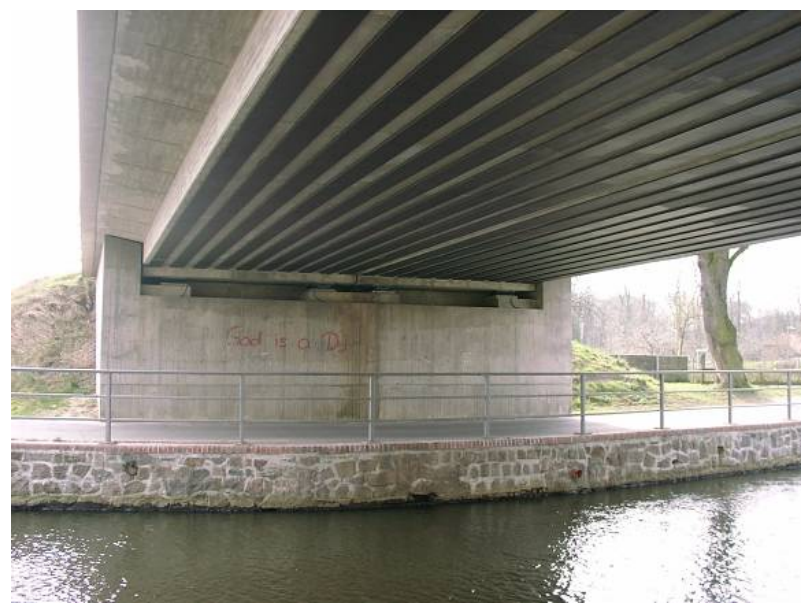

b)

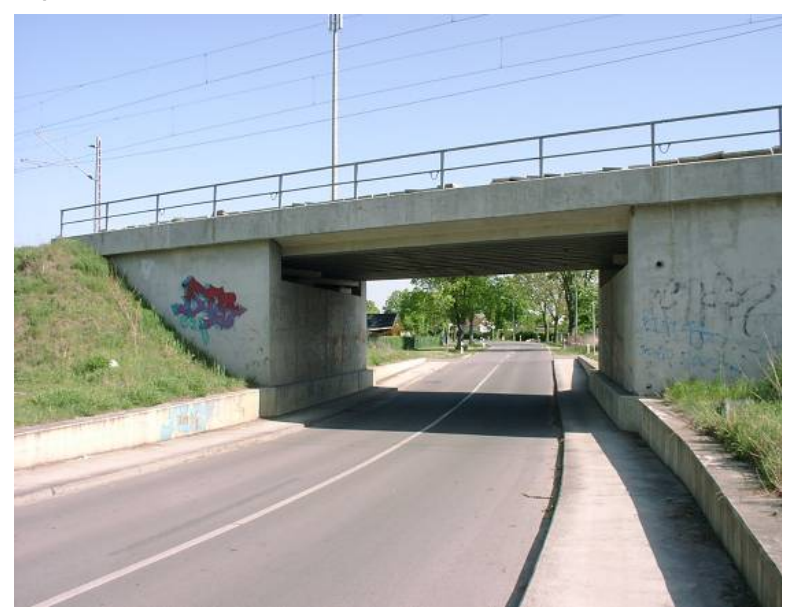

Bild 2.2: WIB-Eisenbahnbrücken a) EÜ Hertefelder Straße im Zuge der HGV-Strecke Hamburg-Berlin b) EÜ Eldekanal im Zuge der HGVStrecke Hamburg-Berlin

Die Beliebtheit der WIB-Bauweise ist wie bereits erwähnt vor allem auf ihr einfaches und schnelles Bauverfahren zurückzuführen, denn eine Einrüstung kann in der Regel entfallen und der Schalungsaufwand ist gering. Auf den Unterflanschen verlegte Faserzementplatten dienen als verlorene Schalung, während die seitliche Schalung der Randflächen und Kragarme an den Walzträgern befestigt wird.

Bild 2.3 zeigt einen typischen WIB-Querschnitt. Einbetonierte Standard-Walzprofile, von denen im Endzustand nur die Unterflansche sichtbar sind, bilden zusammen mit dem umgebenden Beton einen Vollquerschnitt. In der Regel werden WIB-Überbauten schwimmend auf Elastomerlagern gelagert. Methoden zur Bestimmung von Querschnittswerten und zur Berechnung von plastischen Grenztragfähigkeiten sowie Konstruktionsregeln und Angaben zu konstruktiver Mindestbewehrung sind im Anhang K des DIN-Fachberichtes 104 [12] und in Modul 804.4302 der Ril 804 [8] zu finden.

Die Bauweise gehört zur Stahl-Verbundbauweise, wobei die Träger ausschließlich über den Profilverbund mit dem Beton zusammenwirken. Zusätzliche Verbundmittel wie z.B. Kopfbolzendübel sind nicht erforderlich. Die Lastquerverteilung wird durch unten liegende Querbewehrung sichergestellt, die durch Bohrlöcher in den Stegen der Walzprofile geführt wird. Wie in Bild 2.3 zu erkennen, werden in zweigleisigen Strecken häufig zwei getrennte eingleisige Überbauten 
hergestellt. So können bei einer eventuellen Erneuerung die Überbauten nacheinander ersetzt werden, ohne dass während der Bauzeit beide Gleise gleichzeitig gesperrt werden müssen. Die Schotterschicht wird in jedem Fall durchgehend angeordnet, was eine Koppelwirkung der beiden Überbauten bewirkt und, wie später gezeigt wird, signifikante Auswirkungen auf das dynamische Verhalten der Überbauten hat.

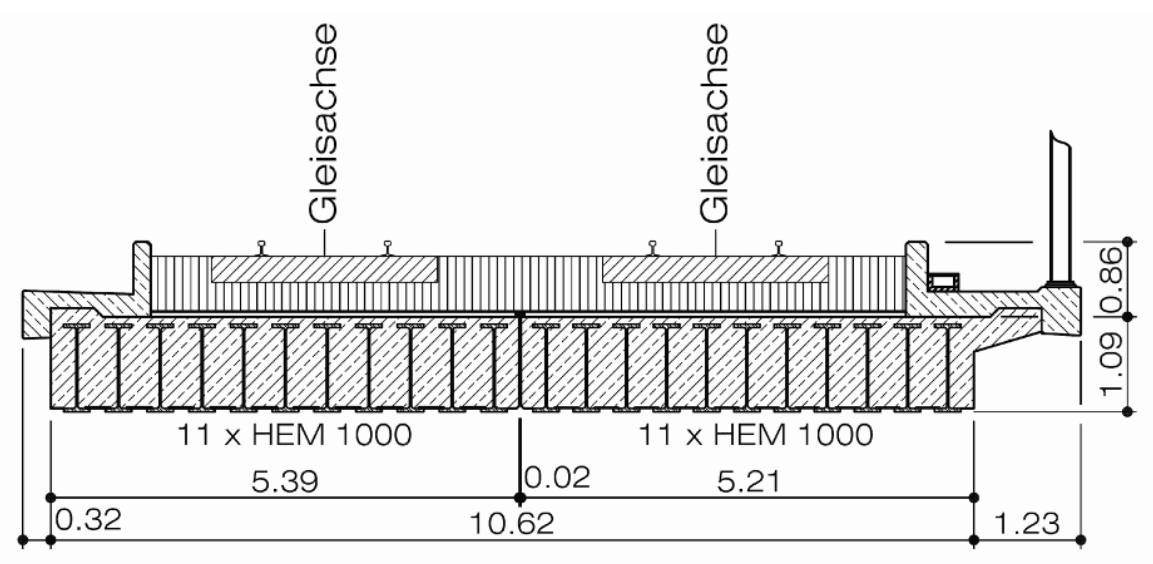

Bild 2.3: Querschnitt einer typischen WIB-Eisenbahnbrücke; zwei durch eine Fuge getrennte Überbauten mit durchgehendem Schotterbett

Durch das Einbetonieren von Stahl-Walzprofilen werden bei WIB-Überbauten hohe Tragfähigkeiten verbunden mit großen Biegeschlankheiten erzielt. Eine Dimensionierung des WIB-Querschnitts erfolgt im Rahmen der Bemessung so, dass die Biegesteifigkeit ausreichend hoch ist, um die notwendige Verformungsbegrenzung zu gewährleisten. Bedingt durch die Einfeldbauweise kann allerdings die erste Biegeeigenfrequenz, die von großer Bedeutung für das Schwingverhalten während Zugüberfahrten ist, bei üblichen Querschnittsmassen so klein werden, dass eine Resonanzanregung des Systems durch die Züge, verbunden mit bemessungsrelevanten Beanspruchungen im SLS und ULS, auftreten kann.

\subsection{Dynamik und dynamische Bemessung von Eisenbahn- brücken für den Hochgeschwindigkeitsverkehr}

Zugtypen für den Hochgeschwindigkeitsverkehr wie beispielsweise die ICE-, TGV- oder ETR-Baureihen, sind durch ihre regelmäßigen Achskonfigurationen und annähernd identischen Achslasten gekennzeichnet. Bei einer Brückenüberfahrt ergibt sich dadurch eine periodische Systemanregung mit der Erregerfrequenz $\mathrm{n}_{\mathrm{E}}$, die von der Wagenlänge $\mathrm{L}_{\ddot{u} \mathrm{P}}$ (Abstand der Fahrgestelle) und der Zuggeschwindigkeit $v$ abhängt. 


$$
n_{E}=\frac{v}{L_{\ddot{u} P}}
$$

Während der Zugüberfahrt antwortet die Brücke nahe der Erregerfrequenz, nach Beendigung der Überfahrt erfolgt ein freies Ausschwingen - im Wesentlichen in ihrer ersten Biegeeigenform. Stimmt die Erregerfrequenz oder ein Vielfaches davon mit einer Systemeigenfrequenz einigermaßen überein, kann es zu einer Resonanzanregung kommen, wobei hauptsächlich eine Anregung in der Grundeigenfrequenz $n_{0}$ von praktischer Bedeutung ist. Gemäß nachstehendem Ausdruck lassen sich zugspezifische kritische Geschwindigkeiten ableiten (vgl. Bild 2.4):

$$
i \cdot n_{E}=i \cdot \frac{v}{L_{u ̈ P}}=n_{0} \Rightarrow v=v_{\text {crit }}=\frac{n_{0} \cdot L_{\ddot{u} P}}{i}
$$

Werte größer als vier sind für den Parameter i gemäß [8] nicht mehr zu berücksichtigen. Er gibt an, nach wie vielen Schwingungsperioden eine erneute Anregung in Phase mit der Brückenschwingung durch eine überfahrende Achsgruppe erfolgt. Für $\mathrm{i}=1$ ist der Energieeintrag in das Schwingungssystem für den jeweiligen Biegeeigenspannungszustand naturgemäß am größten, so dass in

\begin{tabular}{|c|c|c|c|c|c|c|}
\hline \multicolumn{3}{|c|}{ 1. Brückeneigenfrequenz $\left(\mathrm{n}_{0}\right)$} & 3,50 & \multicolumn{3}{|c|}{$v_{c i t}=\frac{n_{0} \cdot L_{i \vec{P} P}}{}$} \\
\hline Zugtyp & ICE1 & ICE3 & Thalys 2 & IC & RE & \\
\hline $\mathrm{L}_{\text {üP }}$ & 26,4 & 24,8 & 18,7 & 26,4 & 26,8 & $\mathrm{~m}$ \\
\hline $\mathbf{v}_{\text {crit }, 1}$ & 333 & 312 & 236 & 333 & 338 & $\mathrm{~km} / \mathrm{h}$ \\
\hline $\mathbf{v}_{\text {crit,2 }}$ & 166 & 156 & 118 & 166 & 169 & $\mathrm{~km} / \mathrm{h}$ \\
\hline $\mathbf{v}_{\text {crit }, 3}$ & 111 & 104 & 79 & 111 & 113 & $\mathrm{~km} / \mathrm{h}$ \\
\hline $\mathbf{v}_{\text {crit } \mathbf{4}}$ & 83 & 78 & 59 & 83 & 84 & $\mathrm{~km} / \mathrm{h}$ \\
\hline
\end{tabular}
diesem Fall die größten Schwingungsamplituden zu erwarten sind.

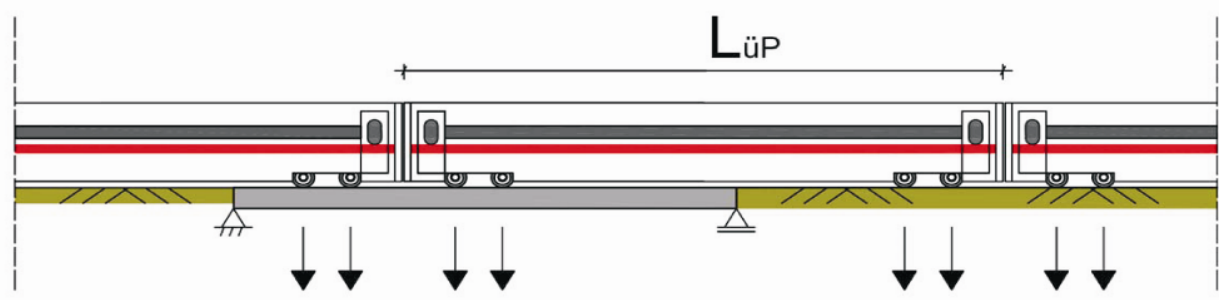

Bild 2.4: Kritische Geschwindigkeiten für verschiedene Zugtypen in Abhängigkeit von der ersten Brückeneigenfrequenz $n_{0}$; Veranschaulichung der „Länge über Puffer“ $L_{\text {üP }}$ 
Die Bedeutung kritischer Geschwindigkeiten für die Bemessung veranschaulicht Bild 2.5. Für zwei Einfeldträger mit identischer Stützweite $(10 \mathrm{~m})$, jedoch unterschiedlichen Biegesteifigkeiten sind die jeweils maximalen Feldmomente aufgetragen, die sich bei ICE-3-Überfahrten mit Geschwindigkeiten von 60 bis $340 \mathrm{~km} / \mathrm{h}$ ergeben und mit Hilfe von numerischen Zeitschrittberechnungen ermittelt wurden. Der Einfluss der Systemdämpfung wurde ebenfalls untersucht, indem die Berechnungen jeweils mit Dämpfungswerten von $1 \%$ und $3 \%$ durchgeführt wurden. Eine geringere Steifigkeit resultiert in einer kleineren Biegeeigenfrequenz. Dadurch ergeben sich für das weichere System verringerte kritische Geschwindigkeiten mit etwa gleich bleibenden maximalen Beschleunigungen und Biegemomenten (vgl. hierzu Bild 5.4). Daneben vergrößern sich infolge der geringeren Steifigkeit die Systemverformungen, wie Bild 2.5 zeigt. Eine höhere Dämpfung führt in erster Linie dazu, dass das Bauwerk mit reduzierten dynamischen Überhöhungen auf die Systemanregung reagiert. Der qualitative Verlauf der Systemantwort und damit auch die kritischen Geschwindigkeiten ändern sich hingegen nicht. Das in Bild 2.5 veranschaulichte Beispiel zeigt, dass die Ergebnisse der dynamischen Analyse erheblich voneinander abweichen können, je nachdem welche Eingangsgrößen für Eigenfrequenz und Dämpfung angesetzt werden.

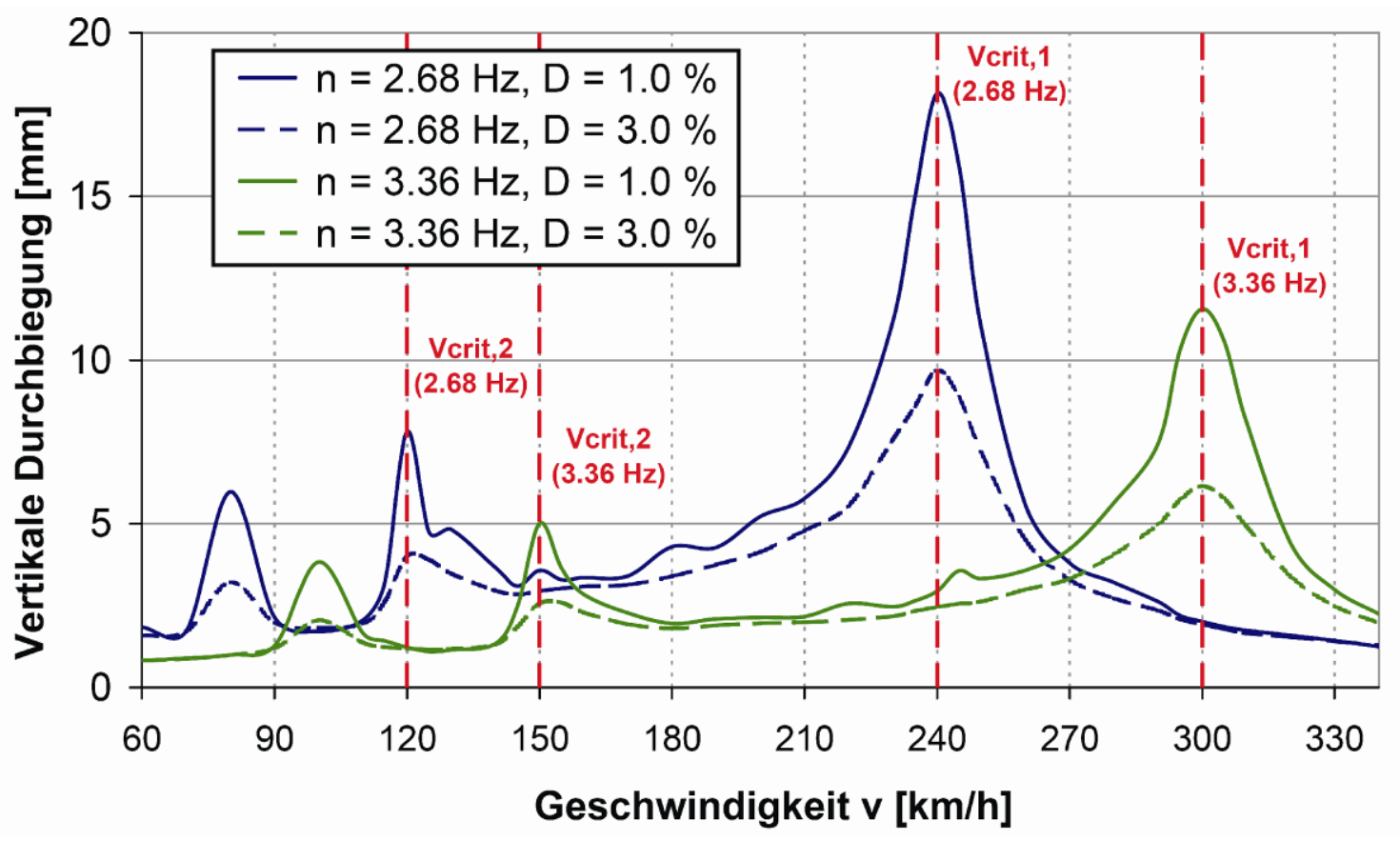

\section{Bild 2.5: Vertikale Durchbiegungen in Feldmitte resultierend aus dyna- mischen Analysen mit unterschiedlichen Randbedingungen}

Für ein dynamisch stabiles System, d.h. ein System, bei dem eine Resonanzanregung ausgeschlossen werden kann, darf eine Bemessung mit statischen Er- 
satzlasten (Lastmodelle LM 71 bzw. SW) und zugehörigen dynamischen Beiwerten auf Grundlage des DIN-Fachberichtes 101 [11] erfolgen. Können Resonanzeffekte hingegen nicht ausgeschlossen werden, was bei Zuggeschwindigkeiten ab $200 \mathrm{~km} / \mathrm{h}$ gemäß [11] generell anzunehmen ist, ist eine signifikante Erhöhung der statischen Ersatzlasten zu erwarten. In diesem Fall ist eine Bemessung mit dynamischen Beiwerten nicht mehr zulässig. Stattdessen muss eine explizite dynamische Berechnung durchgeführt werden, um die Bauwerksbeanspruchungen infolge von Zugüberfahrten zu bestimmen. Die Ergebnisse der dynamischen Berechnung werden sowohl für Nachweise im Grenzzustand der Tragfähigkeit (ULS) als auch im Grenzzustand der Gebrauchstauglichkeit (SLS) benötigt.

- Im ULS ist zu prüfen, ob die aus der dynamischen Analyse resultierenden Beanspruchungen gegenüber den Beanspruchungen infolge der statischen Ersatzlasten maßgebend sind. In diesem Fall sind bei der Nachweisführung die dynamischen Beanspruchungen zu berücksichtigen.

- Die Nachweise im SLS zielen darauf ab, einen ausreichenden Fahrkomfort sicherzustellen sowie verschleißanfällige Brückenelemente wie die Schotterschicht und die Lager zu schützen, um die Instandhaltungskosten gering zu halten. Nachgewiesen werden hierbei Systemverformungen und -beschleunigungen infolge von Zugüberfahrten.

In [33] wird das Vorgehen bei der dynamischen Bemessung in knapper und übersichtlicher Form beschrieben. Eine ausführliche Darstellung der Zusammenhänge ist in [2] zu finden.

Abschließend soll noch auf einen Effekt eingegangen werden, der in der Literatur als "cancellation phenomenon" bezeichnet wird, siehe [32] [37] [65]. Dieser „Aufhebungs-Effekt" bezieht sich auf ein besonderes Ausschwingverhalten von Eisenbahnbrücken nach Zugüberfahrten, die mit einer charakteristischen Geschwindigkeit stattfinden. Diese Geschwindigkeit wird im folgenden $v_{\text {cancel („can- }}$ cellation speed“ bzw. „Aufhebungs-Geschwindigkeit“) genannt.

In Bild 2.6 ist dieses spezielle Ausschwingverhalten dargestellt. Die ausgewählten Zeitreihen stammen aus der in Abschnitt 3.2 behandelten Monitoringkampagne an der WIB-Brücke Erfttalstraße. Während in Bild 2.6b ein Ausschwingen des Systems deutlich zu beobachten ist, verbleibt das System in Bild 2.6a nach Beendigung der Zugüberfahrt annähernd in Ruhe, obwohl die Schwingungsamplituden während der Überfahrt relativ hoch sind. Die Ursache für dieses Verhalten wird im Folgenden kurz erläutert. 
Geht man weiterhin davon aus, dass sich die Belastung durch Zugverkehr hinreichend genau durch eine Abfolge wandernder Einzellasten idealisieren lässt, so ergibt sich die Gesamtantwort des Systems aus der Überlagerung der Einzelantworten. Es wird daher zunächst nur die Anregung des Systems durch eine einzelne wandernde Last betrachtet.

a)

ICE $3-v=205 \mathrm{~km} / \mathrm{h}$

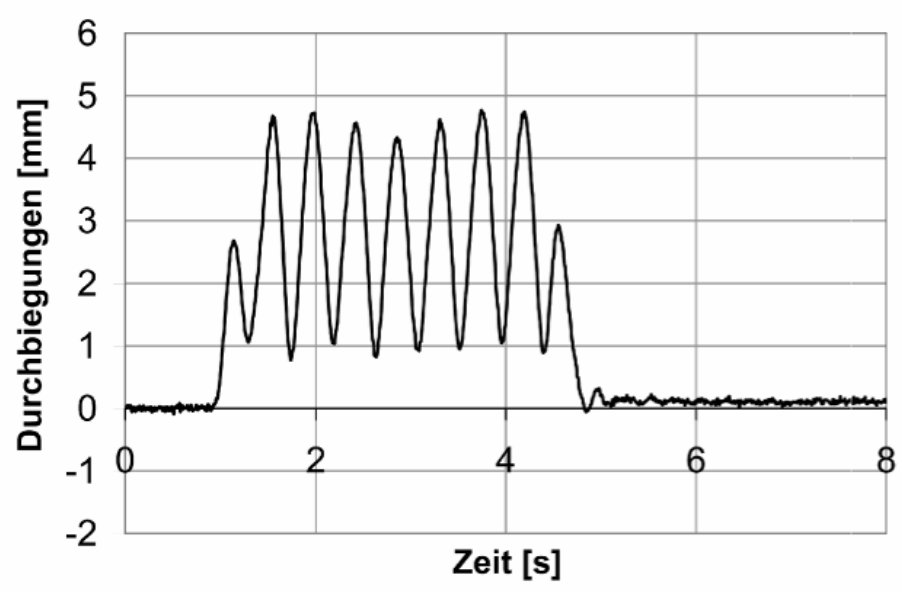

b)

ICE $3-v=155 \mathrm{~km} / \mathrm{h}$

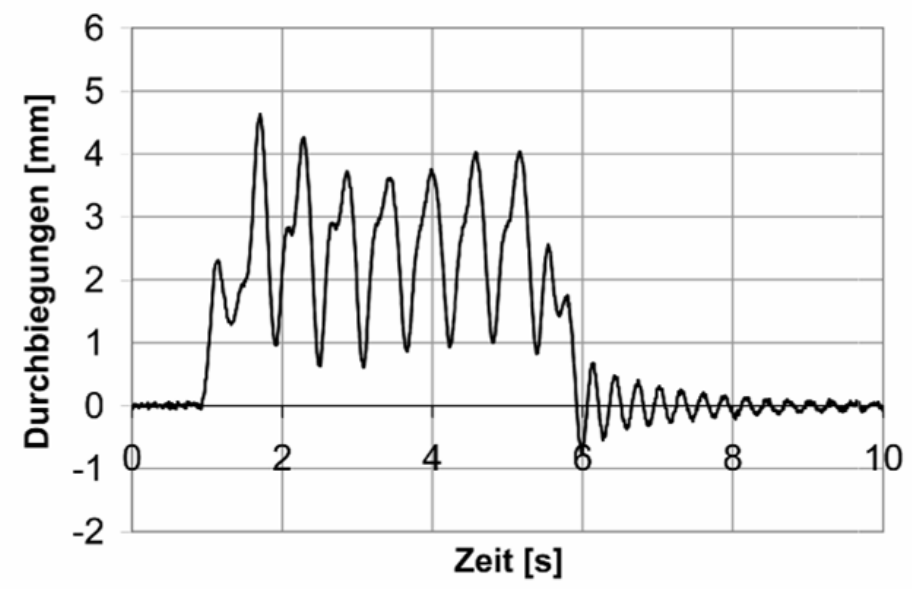

Bild 2.6: Gegenüberstellung des Ausschwingverhaltens (Beschleunigungen in Feldmitte) nach ICE-3 Überfahrten mit a) $203 \mathrm{~km} / \mathrm{h}$ (führt zum Aufhebungseffekt) b) $155 \mathrm{~km} / \mathrm{h}$

Bewegt sich eine wandernde Einzellast mit konstanter Geschwindigkeit v über ein Einfeldsystem mit der Stützweite $L_{s}$, so benötigt sie dafür die Zeit $t_{L s}$ :

$$
t_{L s}=\frac{L_{S}}{v}
$$

Unter der Voraussetzung, dass bei der Zugbeanspruchung eines Einfeldsystems hauptsächlich dessen erste Biegeeigenform angeregt wird, lässt sich die zugehörige Systemantwort gemäß Bild 2.7 darstellen. Sie setzt sich aus einem 
partikulären und einem homogenen Lösungsanteil zusammen. Dabei stellt der partikuläre Anteil die unmittelbare Reaktion auf die einwirkende Kraft dar (,forced vibration“). Unter Berücksichtigung der ersten Biegeeigenform ist der zugehörige Kurvenverlauf halbsinusförmig. Der homogene Anteil (,free vibration") wird durch eine harmonische Schwingung in der zugehörigen Grundeigenfrequenz $\mathrm{n}_{0}$ gebildet und kann als Stoßantwort aufgefasst werden. Auf eine Berücksichtigung des Dämpfungseinflusses wurde in Bild 2.7 vereinfacht verzichtet.

a)

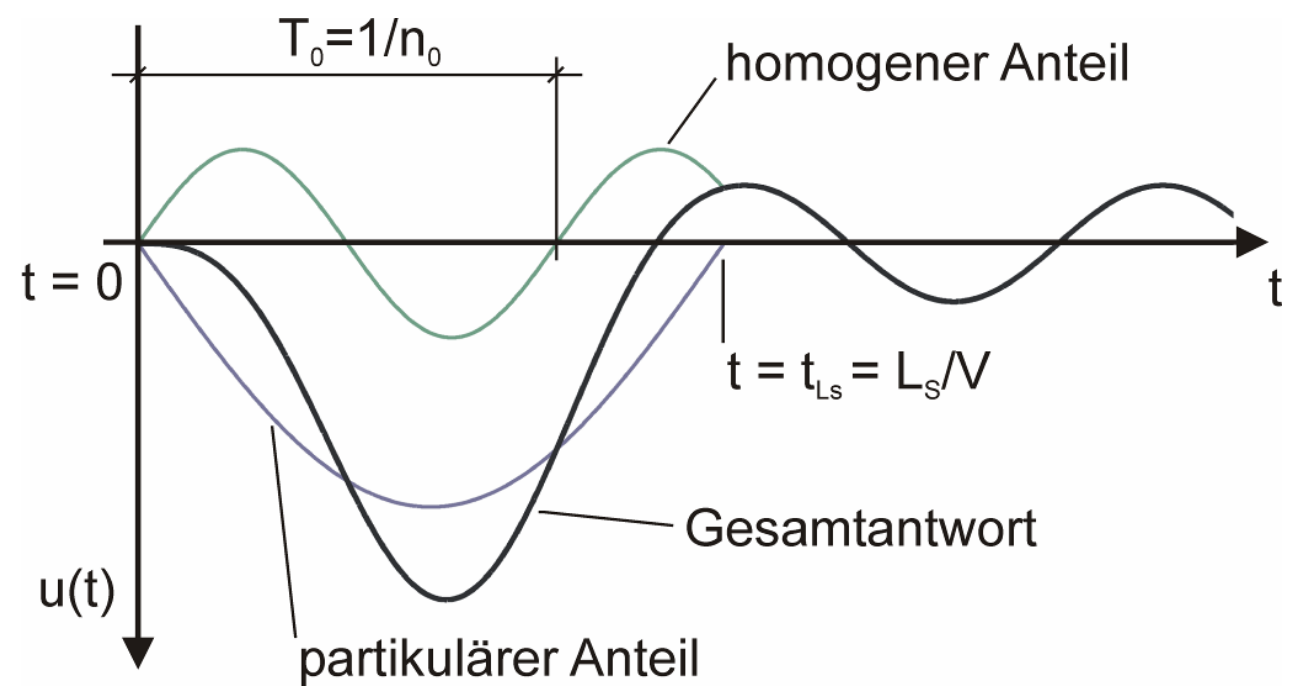

b)

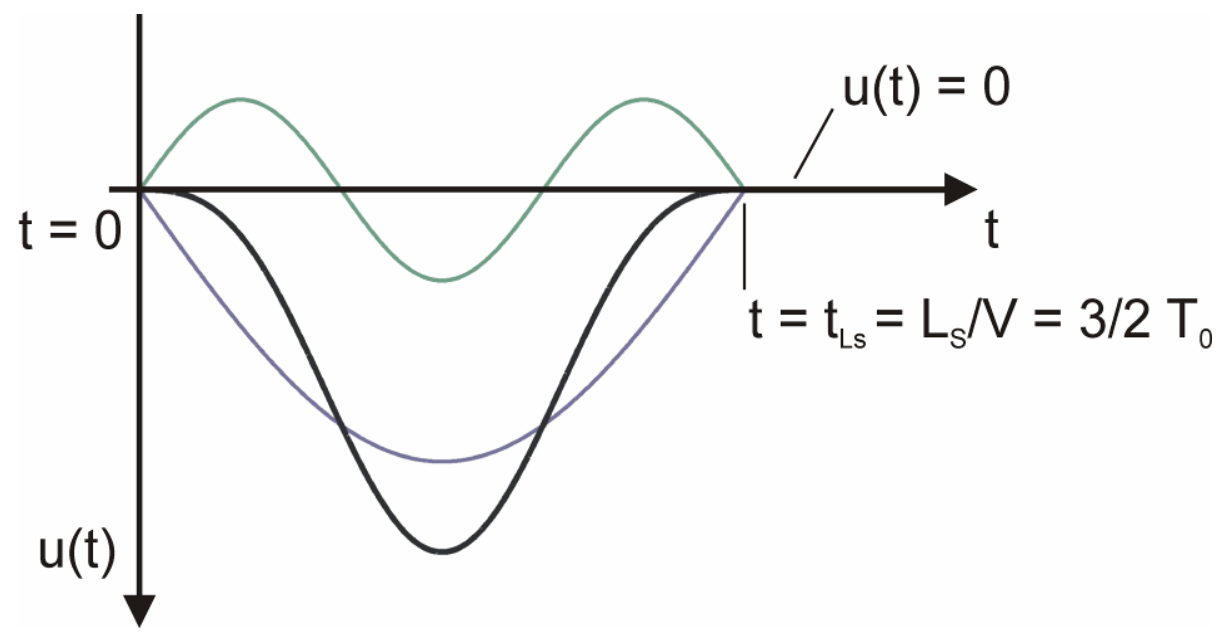

Bild 2.7: Systemantwort auf eine wandernde Einzellast (Durchbiegungen in Feldmitte) als Summe aus partikulärem und homogenem Lösungsanteil unter Berücksichtigung der ersten Eigenform a) resultierend in einer Ausschwingkurve b) Aufhebungseffekt mit $\mathrm{j}=1$

Nach Beendigung des Belastungszeitraums $t_{L s}$ setzt das freie Ausschwingen des Systems ein, vgl. Bild 2.7a. Die Anfangsbedingungen dieses Prozesses er- 
geben sich aus der Summe von partikulären und homogenen Anteilen der entsprechenden Bewegungsgrößen zum Zeitpunkt $t_{L s}$ oder anders ausgedrückt: Die Anfangsamplitude der Ausschwingkurve ergibt sich aus der Addition der Amplituden von partikulärem und homogenem Anteil zum Zeitpunkt $t_{L s}$, die Anfangsgeschwindigkeit ergibt sich aus der Addition der Kurvensteigungen von partikulärem und homogenem Anteil zum Zeitpunkt $t_{\mathrm{Ls}}$.

Sind sowohl Anfangsamplitude als auch -geschwindigkeit der Ausschwingkurve gleich Null, so bleibt das System in Ruhe - homogener und partikulärer Lösungsteil heben sich gegenseitig auf, vgl. Bild 2.7b. Dieser Fall tritt ein, wenn die Amplitude der homogenen Lösung zum Zeitpunkt $t_{L s}$ Null ist und die Steigung der homogenen Lösung den negativen Wert der Steigung der partikulären Lösung annimmt. Dies gilt für

$$
t_{L s}=\frac{2 j+1}{2} \cdot T_{0} \quad j=1,2, \ldots
$$

In Bild 2.7b ist der Zusammenhang für $\mathrm{j}=1$ dargestellt. Für $\mathrm{j}=0$ erhält man einen Sonderfall, bei dem der Aufhebungseffekt nicht auftritt, siehe [37].

Gleichsetzten der Gleichungen (2-3) und (2-4) führt mit $T_{0}=1 / n_{0} z u$

$$
v_{\text {cancel }}=\frac{2}{2 j+1} \cdot n_{0} \cdot L_{S} \quad j=1,2, \ldots
$$

Bei diesen Aufhebungsgeschwindigkeiten bleibt das System also nach Beanspruchung durch die wandernde Einzellast zum Zeitpunkt $t_{L s}$ in Ruhe.

Werden nun zur Bestimmung der Gesamtantwort des Systems auf eine Zugüberfahrt alle zu den einzelnen Achsen gehörigen Einzelantworten überlagert, so folgt daraus, dass das System, nachdem die letzte Achse die Überquerung abgeschlossen hat, ebenfalls in Ruhe bleibt - ein Ausschwingprozess findet infolge des Aufhebungseffektes nicht statt.

Ein Sonderfall ergibt sich, wenn Resonanz- und Aufhebungseffekt gemeinsam auftreten. Setzt man zugspezifische kritische Geschwindigkeiten $v_{\text {crit }}$ - siehe Gleichung (2-2) - und spannweitenabhängige Aufhebungsgeschwindigkeiten $\mathrm{V}_{\text {cancel }}$ - siehe Gleichung (2-5) - entsprechend nachfolgender Gleichung gleich

$$
\frac{n_{0} \cdot L_{\ddot{u} P}}{i}=\frac{2}{2 j+1} \cdot n_{0} \cdot L_{S} \quad i, j=1,2, \ldots
$$

so ergibt sich für das Verhältnis von Stützweite zu Wagenlänge: 


$$
\frac{L_{S}}{L_{\ddot{u} P}}=\frac{2 j+1}{2 i} \quad i, j=1,2, \ldots
$$

wobei die Parameter $\mathrm{i}$ und $\mathrm{j}$ voneinander unabhängig sind.

Für die aus Gleichung (2-7) ableitbaren Verhältniswerte erhält man also den Sonderfall, dass die Resonanzanregung des Systems infolge des Aufhebungseffekts unterdrückt wird. Dies erklärt, warum in seltenen Fällen erwartete Resonanzerscheinungen an Hochgeschwindigkeitsbrücken ausbleiben, obwohl die Brücke mit $\mathrm{v}_{\text {crit }}$ befahren wird.

Die vorangegangenen Ausführungen beziehen sich streng genommen nur auf ungedämpfte Systeme. Im allgemeinen Fall eines schwach gedämpften Systems heben sich partikulärer und homogener Anteil zum Zeitpunkt $t_{L s}$ nicht vollständig auf, so dass eine Restschwingung bleibt, vgl. z. B. Bild 2.6a. Allerdings sind die Amplituden dieser Restschwingung so klein, dass unter praktischen Gesichtspunkten von einer Unterdrückung des Ausschwingvorgangs ausgegangen werden kann.

\subsection{Auswirkungen baulicher Randbedingungen auf die Brückendynamik}

Wie im vorherigen Abschnitt gezeigt wurde, sind Grundeigenfrequenz und Dämpfungseigenschaften einer Eisenbahnbrücke von entscheidender Bedeutung für die dynamische Bemessung. Die erste Biegeeigenfrequenz lässt sich für einen balkenartigen Einfeldträger (Bernoulli-Euler-Balken) mit idealgelenkiger Lagerung nach folgender Formel berechnen, wobei $L$ die Stützweite, El die Systemsteifigkeit und $m$ die Massenverteilung je Längeneinheit bezeichnen.

$$
n_{0}=\frac{\pi}{2 \cdot L^{2}} \sqrt{\frac{E I}{m}}
$$

Messungen nicht nur an WIB-Brücken zeigen jedoch, dass rechnerisch ermittelte Eigenfrequenzen von kurzen und mittelweit spannenden Brücken in vielen Fällen deutlich niedriger sind als die tatsächlich vorhandenen Werte (siehe z.B. [33] [43] [50] [57] [61]). Da die Parameter Stützweite und Massenverteilung in GI. (2-8) relativ genau abgeschätzt werden können, kommt als Ursache für die Fehleinschätzung der Eigenfrequenz in Betracht, dass die Systemsteifigkeit zu niedrig angesetzt wird oder dass die angenommenen Systemidealisierungen unzutreffend sind. Verschiedene Effekte können hierfür verantwortlich gemacht werden: 
- Rechnerisch nicht berücksichtigte Steifigkeitsbeiträge von Ober- und Ausbauelementen; dazu zählen Schutzschicht, Randkappen, Schotter und Schienen, ebenso Schallschutzwände, Kabelkanäle u.ä.

- Durch Rückstellkräfte hervorgerufene Einspannwirkung am Auflager in Abhängigkeit von Lagertyp und Auflagergeometrie

- Kopplung von nebeneinander liegenden, baulich getrennten Überbauten infolge kontinuierlicher, d.h. baulich nicht getrennter Schotterschichten

Eine Vorhersage tatsächlich vorhandener Dämpfungseigenschaften ist hinsichtlich ihrer Zuverlässigkeit nicht einfach. In Bild 2.8a sind die in Richtlinie 804 [8] enthaltenen Vorgaben bzgl. der Dämpfungseigenschaften verschiedener Brückentypen dargestellt.

a)

\begin{tabular}{|l|c|c|}
\hline \multirow{2}{*}{ Bauart } & \multicolumn{2}{|c|}{ Lehrsche Dämpfung $\zeta$ [\%] } \\
\cline { 2 - 3 } & $\mathrm{L}<20 \mathrm{~m}$ & $\mathrm{~L} \geq 20 \mathrm{~m}$ \\
\hline $\begin{array}{l}\text { Stahl- und } \\
\text { Verbundbrücken }\end{array}$ & $\zeta=0,5+0,125(20-\mathrm{L})$ & $\zeta=0,5$ \\
\hline Spannbetonbrücken & $\zeta=1,0+0,07$ (20-L) & $\zeta=1,0$ \\
\hline $\begin{array}{l}\text { Stahlbetonbrücken und } \\
\text { Brücken mit einbeto- } \\
\text { nierten Stahlträgern }\end{array}$ & $\zeta=1,5+0,07$ (20-L) & $\zeta=1,5$ \\
\hline $\begin{array}{l}\text { L Stützweite bei Einfeldträgern, für andere Systeme } \mathrm{L}_{\Phi} \\
\text { nach DIN-Fb 101, IV-6.4.3.3 und Tab. 6.2. }\end{array}$ \\
\hline \multicolumn{2}{|c|}{}
\end{tabular}

b)

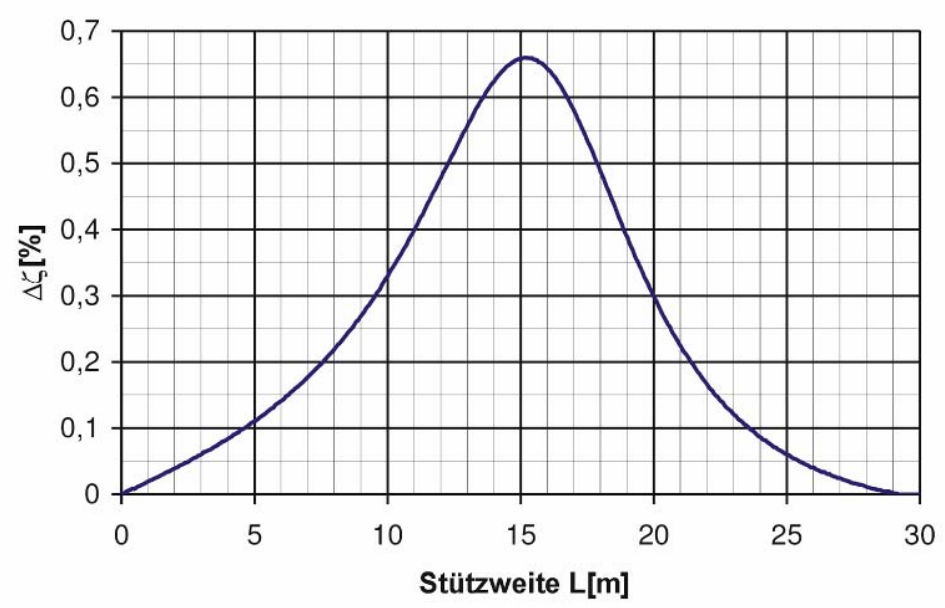

Bild 2.8: Normative Vorgaben für Dämpfungswerte von Eisenbahnbrücken in Abhängigkeit von Bauart und Stützweite [8]: a) Grundwerte b) Zusatzdämpfung bei Überfahrtsimulationen mit bewegten Einzellasten 
Für WIB-Brücken ergeben sich demnach Grundwerte für die Lehr'sche Dämpfung je nach Stützweite zwischen 1,5 und 2,5\%. Hinzu kommt eine von der Stützweite abhängige Zusatzdämpfung, die bei Überfahrtsimulationen angesetzt werden darf, für den Fall dass mit bewegten Einzellasten gerechnet wird (Bild 2.8b). Mit dieser Zusatzdämpfung wird die dämpfende Wirkung des überfahrenden Zuges berücksichtigt.

Die bereits oben angeführten Messergebnisse zeigen, dass die normativ vorgegebenen Werte in vielen Fällen deutlich auf der sicheren Seite liegen. Als ursächlich hierfür werden folgende Faktoren angesehen:

- Erhöhte Dämpfung durch Reibvorgänge im Schotter

- Dämpfungsbeiträge der Oberbau- und Ausrüstungselemente sowie der Lager

Allerdings ist anzumerken, dass die Normung bewusst konservative Dämpfungswerte vorgibt, um eine auf der sicheren Seite liegende dynamische Bemessung sicherzustellen.

Eine Nichtberücksichtigung der baulichen Randbedingungen führt also dazu, dass Systemsteifigkeiten und Systemdämpfung unterschätzt werden. Wie im vorherigen Abschnitt gezeigt wurde, ergeben sich aus dieser Fehleinschätzung im Rahmen einer dynamischen Analyse Bauwerksreaktionen, die ungünstiger sind als das tatsächliche Verhalten. Die Bemessung liegt damit zwar auf der sicheren Seite, allerdings kann die konservative Einschätzung von Eigenfrequenz und Dämpfung, wie unter 1.1 einleitend beschrieben, zu einer deutlichen Einschränkung der Bauwerksnutzung führen, weil hohe Überfahrtgeschwindigkeiten bestimmter Zugtypen auf Basis rechnerischer Untersuchungen nicht zugelassen werden können. Um eine bessere Vorhersage des dynamischen Systemverhaltens von Brücken kurzer bis mittlerer Spannweiten zu ermöglichen, müssen Eigenfrequenz und Dämpfung im Vorfeld der dynamischen Berechnung hinreichend genau abgeschätzt werden. Nur so sind zuverlässige Prognosen auf rechnerischer Basis möglich.

Beispielsweise lassen sich jüngst veröffentlichte modalanalytische Berechnungsverfahren (siehe [2] [17] [25] [43]) nur dann wirkungsvoll einsetzen, wenn die modalen Eigenschaften der untersuchten Bauwerke hinreichend genau bekannt sind. Das prinzipielle Vorgehen zur Ermittlung der Bauwerksantwort infolge einer Zugüberfahrt ist bei den angeführten Verfahren ähnlich: Unter der Annahme, dass die Wechselwirkung zwischen Zug und Brücke sowie die zusätzlichen Wagenmassen vernachlässigt werden können, lässt sich die Zugbeanspruchung mathematisch als eine Folge von bewegten Einzellasten beschrei- 
ben. Sind weiterhin die Voraussetzungen einer über die Trägerlänge konstanten Biegesteifigkeit und Massenverteilung erfüllt, so kann die Bewegungsgleichung in ein System entkoppelter Differentialgleichungen überführt werden, die sich nach Ermittlung der generalisierten Belastungen separat lösen lassen. Auf diese Weise können Zugüberfahrten mit sehr geringem Aufwand simuliert werden. Jedoch haben Eigenfrequenzen und Dämpfungswerte einen erheblichen Einfluss auf das Resultat und sollten daher als Eingangsparameter hinreichend genau bekannt sein.

Anzumerken ist noch, dass bei der Ermittlung von Eigenfrequenz und Dämpfung auf Basis von Bauwerksmessungen Witterungseinflüsse eine nicht zu unterschätzende Rolle spielen. In [4] [21] [33] [66] [67] stellen die Autoren fest, dass Temperatureinflüsse, Feuchtigkeit und Frost einen erheblichen Einfluss auf die Messergebnisse haben. So werden beispielsweise im Sommer deutlich niedrigere Eigenfrequenzen gemessen als im Winter, insbesondere bei gefrorenem Schotterbett. Dieser Effekt kommt besonders bei kurzen Brücken zum Tragen und lässt sich durch die versteifende Wirkung zusammengefrorener Schottersteine erklären.

\subsection{Zeitschrittintegrationsrechungen zur Simulation von Zugüberfahrten}

\subsubsection{Einführung}

Wird ein schwingungsfähiges Tragwerk wie beispielsweise eine Eisenbahnbrücke dynamischer Beanspruchung in Form von Verkehrsbelastung ausgesetzt, führt dies zu einer Anregung des Systems. Das Tragwerk wird dadurch in Schwingung versetzt. Fasst man das Tragwerk, wie bei der FE-Modellierung der Fall, als diskreten Mehrmassenschwinger auf, so liefert das vektorielle Gleichgewicht zwischen Trägheits-, Dämpfungs-, Rückstellkräften und der äußeren Belastung das lineare Differentialgleichungssystem zweiter Ordnung

$$
\underline{M} \cdot \underline{\ddot{u}}+\underline{C} \cdot \underline{\dot{u}}+\underline{K} \cdot \underline{u}=\underline{F}
$$

mit dem Vektor $\underline{u}$ der zeitabhängigen Verschiebungen (bzw. der ersten und zweiten Ableitung von $\underline{u}$ für Geschwindigkeiten und Beschleunigungen), der Steifigkeitsmatrix $\underline{K}$, der Massenmatrix $\underline{M}$ und der Dämpfungsmatrix $\underline{\underline{C}}$. Der Vektor $\underline{F}$ enthält die äußeren Lasten und ist ebenfalls zeitabhängig. Die Vektoren haben $\mathrm{n}$ Komponenten entsprechend den $\mathrm{n}$ Freiheitsgraden des diskreten Systems, und die Matrizen K, M und C sind quadratische (n,n)-Matrizen. 
Zur numerischen Lösung dieses Differentialgleichungssystems werden üblicherweise zwei Methoden angewandt: direkte Integrationsverfahren und Verfahren, die auf modaler Superposition basieren, also eine Modalzerlegung im Vorfeld der numerischen Integration erfordern. Zwar sind die direkten Integrationsverfahren rechenintensiver als die modalanalytischen Verfahren, weil das in (2-9) formulierte Problem nicht auf die Lösung einiger weniger entkoppelter Differentialgleichungen reduziert werden kann. Jedoch eignen sich direkte Integrationsverfahren insbesondere zur Lösung nichtlinearer Systeme. Hinzu kommt, dass die üblichen Rechnerleistungen mittlerweile groß genug sind, um mit Hilfe direkter Integrationsverfahren numerische Simulationsrechnungen, wie sie in vorliegender Arbeit behandelt werden, mit vertretbarem zeitlichen Aufwand zu bewältigen.

\subsubsection{Direkte Integrationsverfahren}

Bei Anwendung direkter Integrationsverfahren werden die Ausdrücke in Gleichung (2-9) mittels einer Schritt-für-Schritt-Methode integriert. Der Ausdruck „direkt" bezieht sich darauf, dass vor der numerischen Integration keine Transformation des Differentialgleichungssystems notwendig ist. Mit den Verfahren werden Näherungslösungen für Gleichung (2-9) in diskreten Zeitpunkten erzeugt, die untereinander einen zeitlichen Abstand von $\Delta \mathrm{t}$ aufweisen. Es wird also zu diskreten Zeitpunkten nach Kräftegleichgewicht unter Berücksichtigung von Massenträgkeits- und Dämpfungskräften gesucht. Numerische Methoden, die in der Baustatik angewandt werden, lassen sich damit auch im Rahmen direkter Integrationsverfahren einsetzen.

Ist die Lösung für eine Zeitspanne von 0 bis $T$ gesucht und wird $\mathrm{T}$ in $\mathrm{n}$ gleichgroße Zeitintervalle $\Delta t=T / n$ unterteilt, so ergeben sich Lösungen für die Zeitpunkte $0, \Delta \mathrm{t}, 2 \cdot \Delta \mathrm{t}, 3 \cdot \Delta \mathrm{t}, \ldots, \mathrm{n} \cdot \Delta \mathrm{t}$. Dabei ergibt sich die Systemantwort $\underline{u}_{t+\Delta t}$ zum Zeitpunkt $\mathrm{t}+\Delta \mathrm{t}$ (sowie daraus folgend auch $\underline{\dot{u}}_{t+\Delta t}$ und $\underline{\ddot{u}}_{t+\Delta t}$ ) bei vollständig vorgegebener Anregung $\underline{F}$ rechnerisch aus der Systemantwort zum Zeitpunkt t.

Als explizit wird das Integrationsschema dann bezeichnet, wenn $\underline{u}_{t+\Delta t}$ aus dem Kräftegleichgewicht gemäß Gleichung (2-9) zum Zeitpunkt t hervorgeht, siehe nachfolgende Gleichung.

$$
\underline{M} \cdot \underline{\ddot{u}}_{t}+\underline{C} \cdot \underline{\dot{u}}_{t}+\underline{K} \cdot \underline{u}_{t}=\underline{F}_{t}
$$

Wird das Kräftegleichgewicht gemäß Gleichung (2-9) wie beispielsweise beim Newmark-Verfahren hingegen zum Zeitpunkt $t+\Delta t$ formuliert, um zur Lösung 
von $\underline{u}_{t+\Delta t}$ zu gelangen, vgl. Gleichung (2-25), so handelt es sich um ein implizites Integrationsschema.

Genauigkeit, Stabilität und numerischer Aufwand verschiedener Integrationsverfahren richten sich danach, wie groß das Zeitintervall $\Delta \mathrm{t}$ gewählt wird und welche Annahmen für die Verläufe von Verformungen, Geschwindigkeiten und Beschleunigungen in diesem Zeitintervall getroffen werden. Ein Beispiel für die Wahl unterschiedlicher Beschleunigungsverläufe im Zeitintervall $\Delta \mathrm{t}$ wird im nachfolgenden Abschnitt angeführt.

In vorliegender Arbeit werden Zeitschrittberechnungen zur Simulation von Zugüberfahrten auf Eisenbahnbrücken durchgeführt. Die Brückensysteme werden dabei durch den Zugverkehr dynamisch angeregt. Die daraus resultierende Schwingungsantwort setzt sich aus verschiedenen miteinander überlagerten Frequenzanteilen zusammen. Bei diesen Frequenzanteilen handelt es sich zum einen um Erregerfrequenzen, die sich aus den Überfahrtgeschwindigkeiten und den Abständen der Fahrgestelle ergeben, vgl. Gleichung (2-1). Zum anderen handelt es sich um angeregte Systemeigenfrequenzen, wobei die Grundeigenfrequenz in der Regel dominant ist - insbesondere während des Ausschwingvorgangs.

Die einzelnen Frequenzanteile werden mittels direkter Integrationsverfahren unter Verwendung einer einheitlichen Zeitschrittweite $\Delta t$ integriert, d.h. $\Delta t$ ist für alle Frequenzanteile bzw. Schwingungsperioden $T_{i}$ identisch. Für Zeitschrittweiten $\Delta t \leq T_{i} / 10$ lassen sich relativ exakte Lösungen erzeugen. Allerdings lassen sich höhere Frequenzanteile, bei denen $\Delta t$ beispielsweise größer ist als die halbe Schwingungsperiode, sicherlich nicht mehr sinnvoll integrieren. Der o. g. Begriff der Stabilität bezieht sich in diesem Zusammenhang auf die Frage, was bei der Integration von Frequenzanteilen geschieht, für die das Verhältnis $\Delta t / T_{i}$ groß ist. Damit ein Integrationsschema stabil ist, müssen diesbezüglich folgende Bedingungen erfüllt sein:

a) Schwingungsbeiträge von Frequenzanteilen mit großem Wert $\Delta t / T_{i}$ dürfen nicht künstlich verstärkt werden und die Integration der relevanten niederfrequenten Anteile dadurch unbrauchbar machen.

b) Fehler in Verschiebungen, Geschwindigkeiten und Beschleunigungen zum Zeitpunkt $t$, die beispielsweise aus rechnerbedingten Rundungen resultieren können, dürfen bei der numerischen Integration zur Erzeugung der Lösung zum Zeitpunkt $t+\Delta t$ nicht anwachsen. 
Integrationsschemata, welche die Verwendung einer Zeitschrittweite $\Delta t$ erfordern, die kleiner ist als eine kritische Zeitschrittweite $\Delta t_{\text {crit, }}$, werden „bedingt stabil“ genannt. Hingegen ist bei „unbedingt stabilen“ Integrationsschemata die numerische Stabilität unabhängig von der Zeitschrittweite $\Delta \mathrm{t}$ gewährleistet. Frequenzanteile, die aufgrund eines zu großen Verhältnisses $\Delta t / T_{i}$ nicht mehr integriert werden können, sind dann nicht in der numerischen Lösung enthalten.

Allerdings geht die Wahl einer zu großen Zeitschrittweite $\Delta t$ auf Kosten der Genauigkeit. Dabei werden die folgenden beiden Formen von Ungenauigkeit unterschieden, wobei sich der Begriff der Ungenauigkeit auf Abweichungen von der exakten analytischen Lösung bezieht.

a) Vergrößerung der exakten Schwingungsperioden

b) Abminderung der exakten Schwingungsamplituden

Das zweite Phänomen wird als „numerische Dämpfung“ bezeichnet. Sie führt dazu, dass Frequenzanteile, die zu groß sind, um mit der gewählten Zeitschrittweite hinreichend genau integriert zu werden, aus der Lösung "herausgefiltert" werden. Dieser Effekt kann durchaus gewünscht sein. Allerdings liefert die im nächsten Abschnitt behandelte Newmark-Konstante-Beschleunigungs-Methode, die im Rahmen der vorliegenden Arbeit verwendet wurde, keinen numerischen Dämpfungsbeitrag. Sie ist somit im Hinblick auf die berechneten Schwingungsamplituden exakt.

Vor dem Hintergrund, dass die Gesamtanzahl der Zeitschritte $n \cdot \Delta t$, die zur Lösung des dynamischen Problems benötigt werden, direkt proportional zur Anzahl der erforderlichen Rechenoperationen und damit zur Dauer der Berechnung ist, stellt die Wahl der Zeitschrittweite $\Delta$ t einen Kompromiss dar. Einerseits sollte $\Delta$ t klein genug gewählt werden, um eine ausreichende Genauigkeit zu erzielen und im Falle eines bedingt stabilen Verfahrens die numerische Stabilität sicherzustellen. Andererseits sollte $\Delta t$ nicht zu klein sein, weil die Berechungen sonst unverhältnismäßig viel Zeit in Anspruch nehmen.

Welche Überlegungen die Grundlage bei der Wahl der Zeitschrittweite für die Zeitschrittberechnungen bildeten, die im Rahmen der vorliegenden Arbeit durchgeführt wurden, wird im nächsten Abschnitt erläutert. Weiterführende Ausführungen zu verschiedenen direkten Integrationsverfahren und damit verknüpften Fragen der Stabilität und Genauigkeit können z. B. [3] und [26] entnommen werden. 


\subsubsection{Zeitschrittintegrationsrechnungen mit InfoCAD}

Im Rahmen der Arbeit wurde zur Simulation von Zugüberfahrten die Software InfoCAD [27] verwendet. In diesem kommerziellen Programm, das von vielen Ingenieurbüros in Deutschland standardmäßig zur dynamischen Bemessung von Eisenbahnbrücken verwendet wird, ist zur Integration der Bewegungsgleichungen das allgemeine Integrationsschema nach Newmark und Wilson (mit $\delta=1 / 2$ und $\beta=1 / 4$, siehe unten) implementiert. Allgemein wird bei diesem Verfahren die Lösung zum Zeitpunkt $t+\Delta t$ wie folgt bestimmt:

$$
\begin{aligned}
& \underline{\dot{u}}_{t+\Delta t}=\underline{\dot{u}}_{t}+\int_{t}^{t+\Delta t} \underline{\ddot{u}}(\tau) d \tau \\
& \underline{u}_{t+\Delta t}=\underline{u}_{t}+\underline{\dot{u}}_{t} \cdot \Delta t+\int_{t}^{t+\Delta t} \underline{\ddot{u}}(\tau) \cdot(t+\Delta t-\tau) d \tau
\end{aligned}
$$

Je nachdem, welche Annahme für den Verlauf der Beschleunigungen zwischen den Zeitpunkten $t$ und $t+\Delta t$ getroffen wird, sind im Wesentlichen zwei Methoden zu unterscheiden, vgl. hierzu Bild 2.9:

a) Konstante-Beschleunigungs-Methode

b) Lineare-Beschleunigungs-Methode

a)

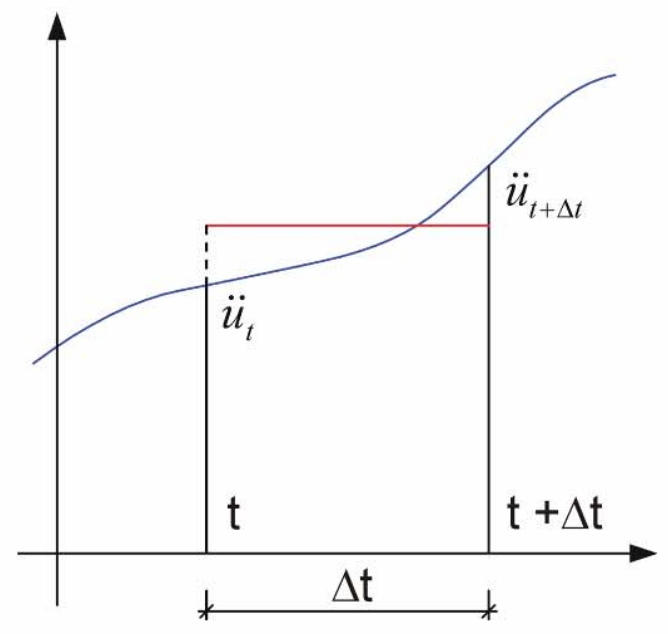

b)

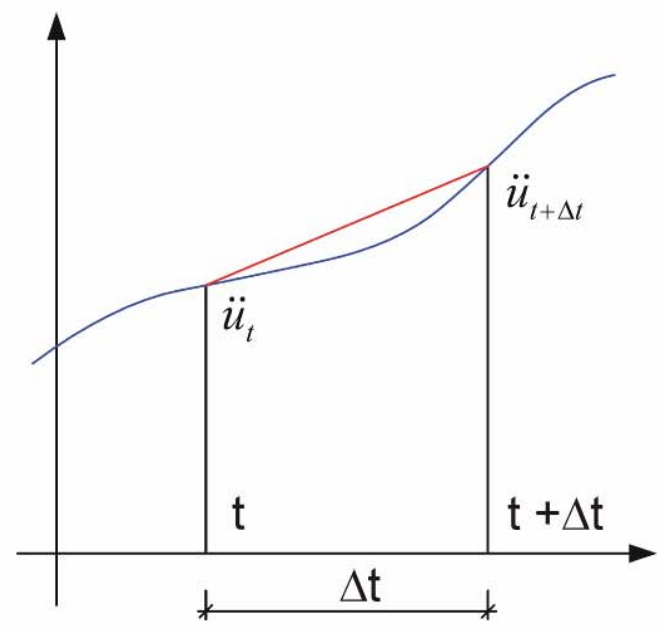

Bild 2.9: Annahmen für den Beschleunigungsverlaufs beim NewmarkVerfahren a) Konstante-Beschleunigungs-Methode b) Lineare Beschleunigungs-Methode 
Bei Methode a wird für einen Zeitpunkt $t+\tau$ mit $0 \leq \tau \leq \Delta t$ eine konstante Beschleunigung angenommen, die dem Mittelwert der Beschleunigungswerte zu den Zeitpunkten $\mathrm{t}$ und $\mathrm{t}+\Delta \mathrm{t}$ entspricht:

$$
\begin{aligned}
& \underline{\ddot{u}}_{t+\tau}=\frac{1}{2} \cdot\left(\underline{\ddot{u}}_{t}+\underline{\ddot{u}}_{t+\Delta t}\right) \\
& \ddot{u}_{t+\tau}=\frac{1}{2} \cdot\left(\ddot{u}_{t}+\ddot{u}_{t+\Delta t}\right)
\end{aligned}
$$

Eine numerische Auswertung der in (2-11) und (2-12) angeführten Ausdrücke führt unter Verwendung von (2-13) zu

$$
\underline{\dot{u}}_{t+\tau}=\underline{\dot{u}}_{t}+\frac{1}{2} \cdot \tau \cdot\left(\underline{\ddot{u}}_{t}+\underline{\ddot{u}}_{t+\Delta t}\right)
$$

und

$$
\underline{u}_{t+\tau}=\underline{u}_{t}+\underline{\dot{u}}_{t} \cdot \tau+\frac{1}{4} \cdot \tau^{2}\left(\underline{\ddot{u}}_{t}+\underline{\ddot{u}}_{t+\Delta t}\right)
$$

Mit (2-15) und (2-16) erhält man zum Zeitpunkt $t+\Delta t$

$$
\begin{aligned}
& \underline{\dot{u}}_{t+\Delta t}=\underline{\dot{u}}_{t}+\frac{1}{2} \cdot \Delta t \cdot\left(\underline{\ddot{u}}_{t}+\underline{\ddot{u}}_{t+\Delta t}\right) \\
& \underline{u}_{t+\Delta t}=\underline{u}_{t}+\underline{\dot{u}}_{t} \cdot \Delta t+\frac{1}{4} \cdot \Delta t^{2}\left(\underline{\ddot{u}}_{t}+\underline{\ddot{u}}_{t+\Delta t}\right)
\end{aligned}
$$

was sich auch allgemein schreiben lässt als

$$
\begin{aligned}
& \underline{\dot{u}}_{t+\Delta t}=\underline{\dot{u}}_{t}+\Delta t \cdot\left[(1-\delta) \cdot \underline{\ddot{u}}_{t}+\delta \cdot \underline{\ddot{u}}_{t+\Delta t}\right] \\
& \underline{u}_{t+\Delta t}=\underline{u}_{t}+\underline{\dot{u}}_{t} \cdot \Delta t+\Delta t^{2} \cdot\left[\left(\frac{1}{2}-\beta\right) \cdot \underline{\ddot{u}}_{t}+\beta \cdot \underline{\ddot{u}}_{t+\Delta t}\right]
\end{aligned}
$$

mit $\delta=1 / 2$ und $\beta=1 / 4$.

Wird wie bei Methode $b$ für einen Zeitpunkt $t+\tau$ mit $0 \leq \tau \leq \Delta t$ ein linearer Verlauf der Beschleunigungen zwischen den Zeitpunkten $t$ und $t+\Delta t$ angenommen gemäß

$$
\underline{\ddot{u}}_{t+\tau}=\underline{\ddot{u}}_{t}+\frac{\tau}{\Delta t} \cdot\left(\underline{\ddot{u}}_{t+\Delta t}-\underline{\ddot{u}}_{t}\right)
$$




$$
\ddot{u}_{t+\tau}=\ddot{u}_{t}+\frac{\tau}{\Delta t} \cdot\left(\ddot{u}_{t+\Delta t}-\ddot{u}_{t}\right)
$$

so führt die numerische Integration der Ausdrücke in (2-11) und (2-12) entsprechend (2-15) und (2-16) zu

$$
\begin{aligned}
& \dot{\dot{u}}_{t+\Delta t}=\underline{\dot{u}}_{t}+\frac{1}{2} \cdot \Delta t \cdot\left(\underline{\ddot{u}}_{t}+\underline{\ddot{u}}_{t+\Delta t}\right) \\
& \underline{u}_{t+\Delta t}=\underline{u}_{t}+\underline{\dot{u}}_{t} \cdot \Delta t+\Delta t^{2}\left(\frac{1}{3} \underline{\ddot{u}}_{t}+\frac{1}{6} \ddot{\ddot{u}}_{t+\Delta t}\right)
\end{aligned}
$$

wobei (2-17) und (2-23) übereinstimmen. (2-23) und (2-24) lassen sich wiederum allgemein unter Verwendung der Gleichungen (2-19) und (2-20) ausdrücken, wobei nun $\delta=1 / 2$ und $\beta=1 / 6$.

Das Newmark-Verfahren ist ein implizites Integrationsverfahren. Um die gesuchte Lösung für $\underline{u}_{t+\Delta t}$ zu erhalten, wird (2-9) zum Zeitpunkt $t+\Delta t$ angeschrieben gemäß

$$
\underline{M} \cdot \underline{\ddot{u}}_{t+\Delta t}+\underline{C} \cdot \underline{\dot{u}}_{t+\Delta t}+\underline{K} \cdot \underline{u}_{t+\Delta t}=\underline{F}_{t+\Delta t}
$$

Indem (2-20) nach $\underline{\ddot{u}}_{t+\Delta t}$ aufgelöst und in (2-19) eingesetzt wird, erhält man Ausdrücke für $\underline{\ddot{u}}_{t+\Delta t}$ und $\underline{\dot{u}}_{t+\Delta t}$, die als einzige Unbekannte jeweils $\underline{u}_{t+\Delta t}$ enthalten. Diese beiden Ausdrücke für $\underline{\ddot{u}}_{t+\Delta t}$ und $\underline{\dot{u}}_{t+\Delta t}$ werden in (2-25) eingesetzt, so dass anschließend die Lösung für $\underline{u}_{t+\Delta t}$ berechnet werden kann. Unter Verwendung von (2-19) und (2-20) können dann $\underline{\ddot{u}}_{t+\Delta t}$ und $\underline{\dot{u}}_{t+\Delta t}$ ebenfalls berechnet werden.

Während Methode b - die "Lineare Beschleunigungs-Methode“ - nur bedingt stabil ist, d.h. dass Bedingungen an die Zeitschrittweite $\Delta$ t gestellt werden, um numerische Stabilität sicherzustellen, ist Methode a - die „KonstanteBeschleunigungs-Methode“ - unbedingt stabil, d. h. unabhängig von der Zeitschrittweite $\Delta \mathrm{t}$.

In InfoCAD wird die "Konstante-Beschleunigungs-Methode“ mit $\delta=1 / 2$ und $\beta=1 / 4$ verwendet. Somit wird die numerische Stabilität der dynamischen Berechnungen nicht durch die Wahl der Zeitschrittweite beeinflusst. Wie im vorangegangenen Abschnitt erläutert, muss die Wahl von $\Delta t$ damit vor dem Hinter- 
grund einer gewünschten Genauigkeit bei gleichzeitig akzeptabler Rechendauer stattfinden.

Die Festlegung von $\Delta t$ für die Zeitschrittberechnungen, die im Rahmen der vorliegenden Arbeit durchgeführt wurden, erfolgte auf Grundlage der folgenden Überlegungen:

a) Welche Frequenzanteile sind von wesentlicher Bedeutung für die untersuchten dynamischen Prozesse und sollten daher hinreichend genau numerisch integriert werden?

b) Welche Genauigkeit ist akzeptabel im Hinblick auf die Zielsetzung der numerischen Untersuchungen und somit hinreichend?

Die Erregerfrequenzen, die bei den simulierten Zugüberfahrten auftraten, waren gemäß Gleichung (2-1) mit $v_{\max } \leq 350 \mathrm{~km} / \mathrm{h}$ und $L_{\ddot{u ̈ P, m i n}} \geq 18,7 \mathrm{~m}$ (Thalys) nicht größer als 5,2 Hz. Die maximalen Grundeigenfrequenzen der numerischen Brückenmodelle, die für Überfahrtsimulationen verwendet wurden (vgl. Abschnitte 5.2 bis 5.5), betrugen weniger als $10 \mathrm{~Hz}$. Die zweiten Biegeeigenfrequenzen sowie die ersten Torsionseigenfrequenzen der zweigleisigen bzw. gekoppelten Modelle, die ebenfalls noch nennenswerte Beiträge zu den Schwingungsantworten liefern können, waren nicht größer als etwa $30 \mathrm{~Hz}$. Dieser Wert stimmt mit der Grenzfrequenz nach [8] überein, bis zu der bei der dynamischen Bemessung von Eisenbahnbrücken Frequenzanteile zu berücksichtigen sind. Somit ergab sich für die minimale Schwingungsperiode der als relevant beurteilten Frequenzanteile ein Wert von

$$
T_{\min }=1 / 30=0,033 \mathrm{~s}
$$

In [3] werden in Abhängigkeit des Verhältnisses $\Delta t / T$ zu erwartende Ungenauigkeiten angegeben. Während wie oben beschrieben die Schwingungsamplituden exakt berechnet werden, können bei der numerischen Integration Vergrößerungen der exakten Schwingungsperioden auftreten. Für $\Delta \mathrm{t} / \mathrm{T} \leq 0,15$ sind diesbezüglich maximale Ungenauigkeiten von $6,0 \%$ bis 7,0 \% möglich (Vergrößerung bezogen auf die Schwingungsperiode). Diese Größenordnung wurde als akzeptabel beurteilt, insbesondere vor dem Hintergrund, dass bei den numerische Analysen die Ermittlung maximal auftretender Bauwerksreaktionen im Vordergrund stand. Nicht die Schwingungsdauern, sondern die während der Überfahrten auftretenden Schwingungsamplituden mussten also möglichst exakt bestimmt werden. Es ergab sich damit ein Wert für die maximale Zeitschrittweite von 


$$
t_{\text {max }}=0,033 \cdot 0,15 \cong 0,005 \mathrm{~s}
$$

der im Rahmen der durchgeführten Simulationsrechnungen nicht überschritten wurde.

\subsection{Zum Dämpfungsbeitrag von Eisenbahnschotter}

\subsubsection{Viskose Dämpfung}

Im Rahmen von baudynamischen Berechnungen wird die Dämpfung eines dynamisch beanspruchten Systems üblicherweise durch ein lineares viskoses, d. h. geschwindigkeitsproportionales Dämpfungsmodell abgebildet. Insbesondere bei Behandlung baudynamischer Fragestellungen im Zeitbereich ist dieses Vorgehen vorteilhaft. Das Konzept der viskosen Dämpfung soll am Beispiel eines Einfreiheitsgradschwingers erörtert werden, siehe Bild 2.10.

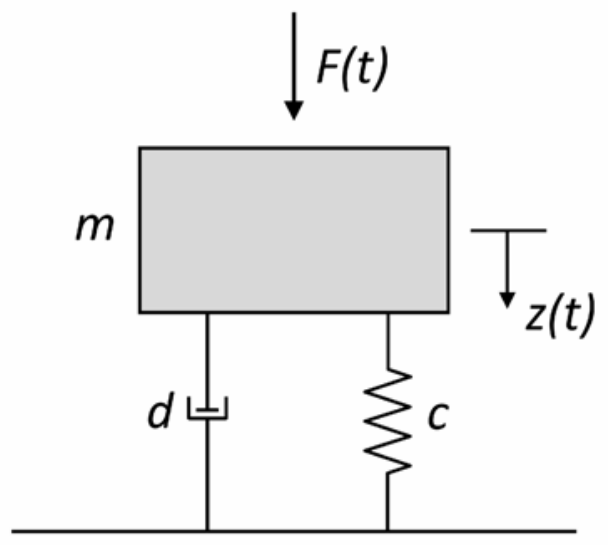

\section{Bild 2.10: Einfreiheitsgradschwinger mit viskoser Dämpfung}

Wird das System harmonisch in einer Frequenz $\Omega$ angeregt, so lautet die Bewegungsgleichung:

$$
m \cdot \ddot{z}+d \cdot \dot{z}+c \cdot z=\hat{F} \cdot \sin (\Omega \cdot t)
$$

Die Dämpfungskraft $F_{D}$ ist darin mit

$$
F_{D, v i s k}=d \cdot \dot{Z}
$$

enthalten. Mit $\mathrm{m}$ wird die schwingende Masse, mit $\mathrm{d}$ die Dämpfungskonstante und mit $c$ die Systemsteifigkeit bezeichnet. $\hat{F}$ ist die Amplitude der harmonischen Belastung. Für die Eigenfrequenz des Systems $\omega$ gilt

$$
\omega^{2}=\frac{c}{m}
$$


Die Schwingungsantwort des Systems ist ebenfalls sinusförmig in der Frequenz $\Omega$. Dieser Schwingungsvorgang lässt sich folgendermaßen formulieren:

$$
z=\hat{z} \cdot \sin (\Omega \cdot t-\alpha)
$$

Dabei ist $\hat{z}$ die Amplitude im eingeschwungenen Zustand und $\alpha$ der aus der Dämpfung resultierende Phasenversatz zwischen Anregung $F(t)$ und Schwingungsantwort $z(t)$. Indem dem schwingenden System durch die Erregerkraft Energie zugeführt wird, wird die Schwingung aufrechterhalten. Erreger- und Massenkraft werden als eingeprägte Kräfte, Dämpfungs- und Federkraft als rückstellende Kräfte bezeichnet.

Die Schwinggeschwindigkeit $\dot{z}$ berechnet sich zu:

$$
\dot{z}=\hat{z} \cdot \Omega \cdot \cos (\Omega \cdot t-\alpha)
$$

Gemäß viskosem Dämpfungsansatz ergibt sich daraus die folgende maximale Dämpfungskraft $F_{D}$ im eingeschwungenen Zustand, siehe auch Bild 2.11. Zu beachten ist hierbei die Frequenzabhängigkeit der Dämpfungskraft.

$$
\max F_{D, v i s k}=d \cdot \hat{z} \cdot \Omega
$$

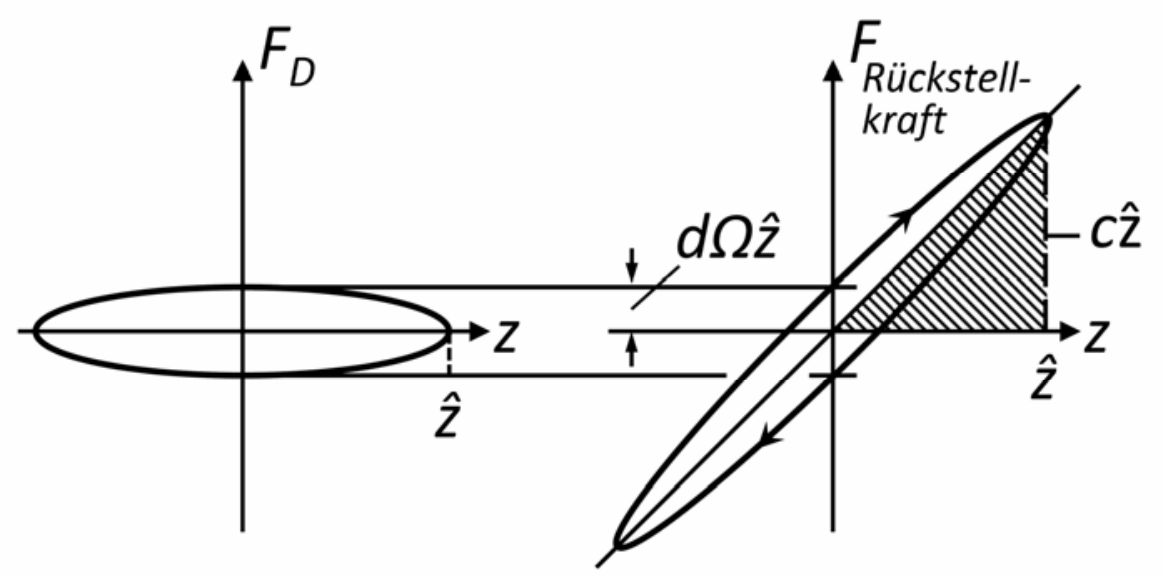

\section{Bild 2.11: Kraft-Verformungszusammenhänge beim viskosen Dämp- fungsmodell nach [40]: elliptische Hysterese des Systems}

Die während eines Schwingungszyklus dissipierte Energie $W_{D}$ berechnet sich als Flächeninhalt der in Bild 2.11 dargestellten elliptischen Hystereseschleife wie folgt:

$$
W_{D, v i s k}=\pi \cdot d \cdot \hat{z}^{2} \cdot \Omega
$$

Die maximale elastisch gespeicherte Arbeit $W_{E}$ beträgt: 


$$
W_{E}=\frac{1}{2} \cdot c \cdot \hat{z}^{2}
$$

Wird $W_{D}$ auf diese Formänderungsarbeit $W_{E}$ bezogen, erhält man die spezifische Dämpfung, die mit $\psi$ abgekürzt wird:

$$
\psi=\frac{W_{D}}{W_{E}}=2 \cdot \pi \cdot \Omega \cdot \frac{d}{c}=2 \cdot \pi \cdot \Omega \cdot \frac{2 \cdot D}{\omega}=4 \cdot \pi \cdot D \cdot\left(\frac{\Omega}{\omega}\right)
$$

Die spezifische Dämpfung ist damit ebenso wie die Dämpfungskraft frequenzabhängig - eine grundlegende Eigenschaft des viskosen Dämpfungsmodells und bei Stoffen mit ausgeprägt visko-elastischem Verhalten tatsächlich zutreffend [40].

\subsubsection{Hysteretische Dämpfung}

Es stellt sich die Frage, ob das viskose Dämpfungsmodell geeignet ist, den Dämpfungsbeitrag des Schotters realistisch abzubilden. In Analogie zu bodendynamischen Vorgängen, bei denen die physikalischen Ursachen der Dämpfung in erster Linie in der Reibung der Bodenkörner aneinander liegen [24], ist bei dynamisch beanspruchten Schotterschichten vermutlich ebenfalls ein Großteil der Energiedissipation auf Reibvorgänge zurückzuführen. Der Energieverlust ist dabei unabhängig von der Verformungsgeschwindigkeit und der Erregerfrequenz [24].

Daher wäre im Falle der schotterbedingten Dämpfung das Konzept der hysteretischen Dämpfung vermutlich dem viskosen Dämpfungsansatz vorzuziehen. Dieses Dämpfungsmodell liefert im Gegensatz zum viskosen Ansatz eine frequenzunabhängige Dämpfungskraft, die der Verformung proportional, ihr gegenüber jedoch um $90^{\circ}$ phasenversetzt ist.

Es bietet sich daher an, zur Formulierung der Dämpfungskraft einen komplexen Ansatz zu wählen, gemäß

$$
F_{D, h y s t}=i \cdot \eta_{V} \cdot c \cdot z
$$

mit der imaginären Einheit i, dem „Verlustfaktor" $\eta_{\vee}$ und der Systemsteifigkeit c. Die Dämpfung eines harmonisch schwingenden Systems lässt sich somit dadurch berücksichtigen, dass die reale Steifigkeit durch eine komplexe ersetzt wird [40]:

$$
c_{\text {ges }}=\left(1+i \cdot \eta_{V}\right) \cdot c
$$


Indem das Problem im Komplexen behandelt wird, bildet die komplexe Steifigkeit neben der elastischen Rückstellwirkung gleichzeitig die Dämpfung des Systems ab. Ist das Problem im Komplexen gelöst, wird die Lösung anschließend ins Reelle rücktransformiert.

Die maximale Dämpfungskraft $F_{D}$ im eingeschwungenen Zustand ergibt sich beim hysteretischen Dämpfungsansatz zu

$$
\max F_{D, v i s k}=\eta_{V} \cdot c \cdot \hat{z}
$$

Damit beträgt die pro Zyklus infolge Dämpfung dissipierte Energie $W_{D}[40]$

$$
W_{D, h y s t}=\pi \cdot \eta_{V} \cdot c \cdot \hat{z}^{2}
$$

Für die Formänderungsarbeit $W_{E}$ gilt wiederum Gleichung (2-35).

Damit ergibt sich für den Fall des hysteretischen Modells die spezifische Dämpfung als Verhältnis von dissipierter Energie pro Schwingungszyklus zur maximal elastisch gespeicherten Arbeit wie folgt:

$$
\psi=\frac{W_{D}}{W_{E}}=2 \cdot \pi \cdot \eta_{V}
$$

Die spezifische Dämpfung ist also bei diesem Modell wie erwartet frequenzunabhängig.

Ein Vergleich mit der Lösung in Gleichung (2-36) lässt erkennen, dass viskoses und hysteretisches Dämpfungsmodell gleichwertig sind, wenn gilt

$$
\eta_{V}=2 \cdot D \cdot\left(\frac{\Omega}{\omega}\right)
$$

Die beiden Modelle können also theoretisch ineinander überführt werden [40]. Insbesondere bei schmalbandigen Prozessen spielt die Art der Formulierung (viskoser oder hysteretischer Ansatz) daher eine untergeordnete Rolle. Solch eine Schmalbandigkeit ist beispielsweise bei der Anregung von Eisenbahnbrücken mit zugtyp- und geschwindigkeitsabhängigen Erregerfrequenzen gegeben.

Gleichung (2-41) gilt strenggenommen nur für stationär-harmonische Schwingungsvorgänge. Daher ist das Konzept der hysteretischen Dämpfung nur auf solche Vorgänge anwendbar [35] [40]. Allerdings wird es beispielsweise bei der Untersuchung bodendynamischer Vorgänge infolge von Erdbebeneinwirkung, bei denen große Verformungen auftreten, verwendet [24]. Es ist durchaus möglich, dass es auch bei der größtenteils monofrequenten Anregung von Eisenbahnbrücken durch lange Züge mit regelmäßigen Achsabständen eingesetzt 
werden kann. Diese Frage sollte jedoch vor der Verwendung des hysteretischen Dämpfungskonzeptes bei Überfahrtsimulationen einer genaueren Betrachtung unterzogen werden.

\subsubsection{Berücksichtigung nichtlinearer Effekte}

Weiterhin von Interesse ist die grundsätzliche Frage nach der Linearität im Hinblick auf den Dämpfungsbeitrag des dynamisch beanspruchten Schotters. Bei den oben behandelten Konzepten handelt es sich um lineare Ansätze. Jedoch wurde im Rahmen der experimentellen Untersuchungen an Eisenbahnschotter, die in Abschnitt 4 behandelt werden, festgestellt, dass sich bei großen Schwingamplituden bzw. großen Relativbewegungen zwischen den einzelnen Schotterkörnern größere Dämpfungswerte einstellen als bei kleinen Amplituden.

Das Phänomen eines nichtlinearen amplitudenabhängigen Dämpfungsbeitrages von Schotterschichten auf Eisenbahnbrücken wurde beispielsweise auch in [30] [51] auf Grundlage experimenteller Untersuchungen beschrieben. Er lässt sich dadurch erklären, dass sich die einzelnen Schotterkörner erst ab einem bestimmten Beanspruchungsgrad gegeneinander verschieben bzw. aneinander reiben. Die dabei geleistete Arbeit der Gleitreibkräfte stellt einen Energieverlust dar, der sich bei einer dynamischen Beanspruchung in Dämpfung ausdrückt.

Um den Reibeffekt mechanisch abzubilden, kann der in Bild 2.10 dargestellte Einfreiheitsgradschwinger um ein Coulombsches Reibelement erweitert werden, siehe Bild 2.3 (zur Modellbildung siehe z. B. [40] [42] [62]). Die vorgeschaltete Feder mit der Federsteifigkeit $\mathrm{C}_{2}$ liefert ein linear-elastisches Kraft-Verformungsverhalten bis zum Erreichen der maximalen Haftreibkraft. Bei weiterer Laststeigerung bleibt die Federkraft konstant, und es findet eine Bewegung im Reibelement statt.

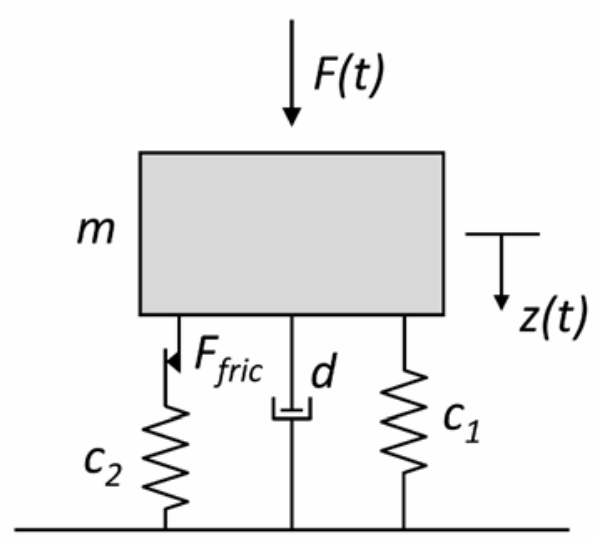

Bild 2.12: Einfreiheitsgradschwinger mit viskoser Dämpfung und Coulombscher Reibung 
Der Coulombsche Reibdämpfer wird also erst nach Überwindung der Haftreibung bzw. der Losreißamplitude $z_{L}$ aktiviert. Für die Beziehung zwischen Gleitreibkraft $F_{\text {fric }}$ und Losreißamplitude gilt unter der Annahme, dass der Maximalwert der Haftreibkraft und die Gleitreibkraft übereinstimmen:

$$
F_{\text {fric }}=z_{L} \cdot c_{2}
$$

Die von der Gleitreibkraft geleistete Arbeit pro Schwingungszyklus (der Weg $\left(\hat{z}-z_{L}\right)$ wird pro Zyklus insgesamt viermal zurückgelegt) bzw. die während eines Schwingungszyklus dissipierte Energie beträgt:

$$
W_{D, \text { fric }}=4 \cdot F_{\text {fric }} \cdot\left(\hat{z}-z_{L}\right)
$$

Bis zur Losreißamplitude $z_{L}$ wird Energie also lediglich infolge viskoser Dämpfung gemäß Gleichung (2-34) dissipiert, bei Überwindung von $z_{L}$ trägt der Reibdämpfer dann ebenfalls zur Energiedissipation bei. Diese nichtlineare Charakteristik ist mit der beobachteten Amplitudenabhängigkeit im Einklang.

Einen ausgeprägten Einfluss auf das Dämpfungsverhalten des Eisenbahnschotters dürfte der Anpressdruck bzw. die Kontaktnormalkraft zwischen den einzelnen Schotterkörnern haben, da die Reibkraft $F_{\text {fric }}$ dieser Kontaktkraft proportional ist. Daher dürfte ein belastetes Schotterbett einer in Schwingung befindlichen Brücke einen deutlich größeren Beitrag zur Systemdämpfung liefern als ein unbelastetes - vorausgesetzt, die Haftreibung zwischen den einzelnen Schotterkörner wird überwunden. Davon ist bei einer Zuganregung mit relativ großen Verformungsamplituden aber auszugehen. Während einer Zugüberfahrt ist daher mit erhöhten Dämpfungswerten zu rechen. Ein experimenteller Nachweis dieses Phänomens wird beispielsweise in [60] geliefert.

\subsection{4 Äquivalente viskose Dämpfung}

Um bei der rechnerischen Behandlung baudynamischer Problemstellungen, bei denen dämpfungsbeeinflussende Reibeffekte eine wichtige Rolle spielen, dennoch auf die üblichen numerischen Methoden zurückgreifen zu können, die in der Regel ein lineares viskoses Dämpfungsmodell voraussetzen, lässt sich eine angenäherte äquivalente viskose Dämpfung als Verhältnis von Arbeitsanteilen bestimmen [35]. Dazu wird Gleichung (2-36) wie folgt umgeformt, um ein äquivalentes Lehrsches Dämpfungsmaß zu bestimmen:

$$
D=\frac{W_{D}}{4 \cdot \pi \cdot W_{E} \cdot\left(\frac{\Omega}{\omega}\right)}=\frac{\psi}{4 \cdot \pi \cdot\left(\frac{\Omega}{\omega}\right)}
$$


$W_{D}$ setzt sich dabei wie erläutert aus viskosem und Coulombschem Anteil zusammen und stimmt dem Betrag nach mit der Arbeit der Erregerkraft pro Schwingungszyklus überein. 


\section{Messungen an WIB-Eisenbahnbrücken}

\subsection{Messkampagnen der Deutschen Bahn}

\subsubsection{Hintergrund}

Im Zuge des Auf- und Ausbaus des Hochgeschwindigkeitsnetzes in Deutschland wurden von der Deutschen Bahn neben der statischen Brückenbemessung auch zahlreiche genauere dynamische Untersuchungen durchgeführt. Dabei waren nicht nur Neubauten sondern auch bestehende Bauwerke Gegenstand der Untersuchungen. In einem ersten Schritt wurden dynamische Berechnungen vorgenommen, um die Auswirkungen des Hochgeschwindigkeitsverkehrs auf Beschleunigungen, Durchbiegungen und maximale Biegemomente abzuschätzen. Wurde ein Brückenbauwerk als problematisch eingestuft bzw. zeigte sich eine Anfälligkeit für Resonanzeffekte, so wurden in einem zweiten Schritt Messungen an dem Brückenbauwerk durchgeführt. Mit Hilfe der Messungen wurden die tatsächlichen Werte von Eigenfrequenz und Dämpfung bestimmt, die anschließend als Eingangsparameter für auf diese Weise kalibrierte dynamische Berechnungen dienten. Unter anderem konnte so die Resonanzgefahr für bestimmte Zugtypen ausgeschlossen werden. Gerade vor dem Hintergrund der Einführung der neuen ICE-3-Züge war dies von besonderer Bedeutung, weil die geringeren Wagenlängen dieses Zugtyps zu anderen Resonanzgeschwindigkeiten führen als bei ICE-1 bzW. ICE-2-Zügen. Im Rahmen der Untersuchungen gerieten besonders WIB-Überbauten in den Fokus, die im HGV-Netz der Deutschen Bahn zahlreich vertreten sind. Mit typischen Spannweiten von 10 bis $25 \mathrm{~m}$ und Biegeschlankheiten L/h zwischen 14 und 26 ergaben sich in vielen Fällen rechnerische Eigenfrequenzen, die im Hinblick auf Resonanzeffekte als kritisch eingeschätzt wurden. Als Konsequenz wurden von der Deutschen Bahn an vielen WIB-Brücken Beschleunigungs- und Verformungsmessungen durchgeführt. Zugehörige Messberichte und Ergebnisprotokolle [6] konnten im Rahmen dieser Arbeit ausgewertet werden.

\subsubsection{Auswertung der Messungen}

Insgesamt wurden Messberichte von rund 30 Brücken in WIB-Bauweise ausgewertet, die sich auf vier verschiedenen zweigleisigen HGV-Strecken befinden [6]. Tabelle 3.1 stellt die ausgewerteten Messungen im Überblick dar. Die Messungen fanden sowohl an eingleisigen Überbauten mit durchgehendem Schotterbett $(2 \times 1 \mathrm{WIB})$ als auch an zweigleisigen Überbauten $(1 \mathrm{WIB})$ statt. In vier Fällen wurde bei eingleisigen Brücken an beiden Überbauten gemessen. Im 
Falle der EÜ Nauen Paretzer Kanal, die aus drei hintereinander angeordneten Einfeldträgern besteht, wurden alle drei Überbauten untersucht. An den Brücken wurden im Wesentlichen Beschleunigungen und Durchbiegungen infolge von Zugüberfahrten sowie Zuggeschwindigkeiten gemessen. Durch Auswertung der Abklingkurven nach Überfahrtsereignissen wurden die tatsächlich vorhandenen Grundeigenfrequenzen und Dämpfungseigenschaften bestimmt.

Tabelle 3.1: Übersicht der untersuchten Brücken mit Messergebnissen

\begin{tabular}{|c|c|c|c|c|c|c|c|c|c|c|c|c|c|c|c|c|c|}
\hline \multirow[b]{2}{*}{ Strecke } & \multirow[b]{2}{*}{ EÜ } & \multirow[b]{2}{*}{ km } & \multirow[b]{2}{*}{ Typ } & \multicolumn{6}{|c|}{ Brückeneigenschaften } & \multicolumn{3}{|c|}{ Eigenfreq. } & \multicolumn{2}{|c|}{ Steifigkeit } & \multicolumn{3}{|c|}{ Dämpfung } \\
\hline & & & & $\begin{array}{l}\mathrm{L} \\
(\mathrm{m})\end{array}$ & $\begin{array}{l}\mathrm{b} \\
(\mathrm{m})\end{array}$ & $\begin{array}{l}\mathrm{h} \\
(\mathrm{m})\end{array}$ & $\begin{array}{l}\mathrm{L} / \mathrm{h} \\
(-)\end{array}$ & $\underset{(t / m)}{m}$ & $\begin{array}{c}\mathrm{EI}_{\mathrm{cal}} \\
\left(\mathrm{MNm}^{2}\right)\end{array}$ & $\begin{array}{l}\mathrm{n}_{0, \text { cal }} \\
(\mathrm{Hz})\end{array}$ & $\begin{array}{l}\mathrm{n}_{0, \mathrm{eff}} \\
(\mathrm{Hz})\end{array}$ & $\begin{array}{l}\text { Abw. } \\
(\%)\end{array}$ & $\begin{array}{c}\mathrm{El}_{\mathrm{eff}} \\
\left(\mathrm{MNm}^{2}\right)\end{array}$ & $\begin{array}{c}E I_{\mathrm{eff}} / \mathrm{El}_{\mathrm{cal}} \\
(-)\end{array}$ & $\begin{array}{l}D_{\text {cal }} \\
(\%)\end{array}$ & $\begin{array}{l}D_{\text {eff }} \\
(\%)\end{array}$ & $\begin{array}{l}\text { Abw. } \\
(\%)\end{array}$ \\
\hline \multirow{7}{*}{$\begin{array}{l}\text { Hannover - } \\
\text { Würzburg }\end{array}$} & Glückaufstr. & 21,84 & $2^{*} \mid \mathrm{WiB}$ & 12,9 & 5,3 & 0,63 & 20,5 & 20,5 & 5161 & 4,7 & 9,4 & 99 & 20378 & 3,95 & 2,0 & 4,9 & 145 \\
\hline & Rössingbach & 24,47 & $1 \mathrm{WiB}$ & 12,9 & 10,4 & 0,75 & 17,2 & 43,6 & 15382 & 5,6 & 8,0 & 43 & 31313 & 2,04 & 2,0 & 4,0 & 100 \\
\hline & OVW Sellenst. & 44,15 & $2 * 1 \mathrm{WiB}$ & 16,0 & 5,1 & 0,86 & 18,6 & 23,3 & 10577 & 4,1 & 5,9 & 43 & 21560 & 2,04 & 1,8 & 4,0 & 125 \\
\hline & Helleweg & 50,19 & $2 * I \mathrm{WiB}$ & 13,0 & 5,2 & 0,90 & 14,4 & 24,8 & 11986 & 6,5 & 8,2 & 27 & 19316 & 1,61 & 2,0 & 4,2 & 112 \\
\hline & Lutterbach & 97,92 & $2 * 1 \mathrm{WiB}$ & 13,2 & 5,5 & 0,63 & 20,9 & 20,7 & 5538 & 4,7 & 8,5 & 81 & 18221 & 3,29 & 2,0 & 3,5 & 77 \\
\hline & Iheringstr. & 100,31 & $2 * 1 \mathrm{WiB}$ & 13,6 & 5,3 & 0,73 & 18,6 & 21,2 & 6778 & 4,8 & 7,0 & 46 & 14388 & 2,12 & 1,9 & 4,0 & 105 \\
\hline & K 72, Neuhof & 245,07 & $2 * 1 \mathrm{WiB}$ & 16,5 & 5,0 & 0,85 & 19,4 & 24,2 & 12903 & 4,2 & 5,9 & 40 & 25346 & 1,96 & 1,7 & 5,0 & 187 \\
\hline \multirow{3}{*}{$\begin{array}{c}\text { Oranienburg - } \\
\text { Neustrelitz }\end{array}$} & Havel & 77,65 & $2^{*} 1 \mathrm{WiB}$ & 22,1 & 4,5 & 1,29 & 17,1 & 25,7 & 34057 & 3,7 & 5,3 & 44 & 70260 & 2,06 & 1,5 & 3,7 & 143 \\
\hline & Steinförder Str. & 77,44 & $2 * I \mathrm{WiB}$ & 10,4 & 4,5 & 0,54 & 19,3 & 16,9 & 2774 & 5,9 & 10,5 & 78 & 8815 & 3,18 & 2,2 & 4,0 & 84 \\
\hline & Grüneberg & 42,30 & $2^{*} 1 \mathrm{WiB}$ & 5,6 & 4,0 & 0,25 & 22,4 & 12,8 & 315 & 9,0 & 19,0 & 111 & 1402 & 4,46 & 2,5 & 5,5 & 119 \\
\hline \multirow{10}{*}{ Köln-Düren } & Goethestr. K-AC & 9,63 & $2^{\circ} 1 \mathrm{WiB}$ & 21,9 & 4,4 & 1,19 & 18,4 & 26,2 & 33598 & 3,7 & 5,0 & 34 & 60463 & 1,80 & 1,5 & 3,8 & 150 \\
\hline & Goethestr. AC-K & 9,63 & $2 * I \mathrm{WiB}$ & 19,4 & 5,0 & 1,19 & 16,3 & 25,5 & 27485 & 4,4 & 7,0 & 61 & 71115 & 2,59 & 1,5 & 5,2 & 236 \\
\hline & B 477 & 27,55 & $2^{* 1} \mathrm{WiB}$ & 15,9 & 5,0 & 0,88 & 18,1 & 22,4 & 11751 & 4,5 & 6,4 & 42 & 23800 & 2,03 & 1,8 & 3,2 & 79 \\
\hline & Rote-Kreuz-Str. & 19,55 & $1 \mathrm{WiB}$ & 20,1 & 10,0 & 0,85 & 23,7 & 48,6 & 26670 & 2,9 & 4,7 & 63 & 70525 & 2,64 & 1,5 & 3,6 & 140 \\
\hline & Erftkanal & 20,02 & $1 \mathrm{WiB}$ & 21,2 & 10,2 & 0,85 & 25,0 & 42,6 & 28636 & 2,9 & 4,4 & 53 & 67147 & 2,34 & 1,5 & 3,0 & 97 \\
\hline & Große Erft & 20,27 & $1 \mathrm{WiB}$ & 19,8 & 10,2 & 0,98 & 20,2 & 44,4 & 36260 & 3,6 & 5,3 & 46 & 77410 & 2,13 & 1,5 & 4,0 & 164 \\
\hline & Herrenstr. AC-K & 21,91 & $2 * 1 \mathrm{WiB}$ & 10,4 & 4,2 & 0,65 & 15,8 & 16,7 & 4336 & 7,5 & 12,9 & 72 & 12886 & 2,97 & 2,2 & 5,0 & 130 \\
\hline & Herrenstr. K-AC & 21,91 & $2^{*} 1 \mathrm{WiB}$ & 11,2 & 5,3 & 0,65 & 17,2 & 19,5 & 5606 & 6,7 & 10,5 & 56 & 13701 & 2,44 & 2,1 & 6,5 & 207 \\
\hline & Erftalstr. AC-K & 21,22 & $2^{* 1} \mathrm{WiB}$ & 24,6 & 5,4 & 1,09 & 22,6 & 26,4 & 29796 & 2,8 & 3,5 & 27 & 47933 & 1,61 & 1,5 & 3,0 & 100 \\
\hline & Erftalstr. $\mathrm{K}-\mathrm{AC}$ & 21,22 & $2 * 1 \mathrm{WiB}$ & 24,6 & 5,2 & 1,09 & 22,6 & 27,8 & 30869 & 2,7 & 3,5 & 28 & 50499 & 1,64 & 1,5 & 2,5 & 67 \\
\hline \multirow{15}{*}{$\begin{array}{c}\text { Hamburg - } \\
\text { Berlin }\end{array}$} & N. Dosse & 76,20 & $2 * 1 \mathrm{WiB}$ & 21,8 & 4,6 & 1,00 & 21,8 & 23,6 & 19843 & 3,0 & 4,5 & 48 & 43286 & 2,18 & 1,5 & - & - \\
\hline & Fauler Graben & 113,68 & $2 * 1 \mathrm{WiB}$ & 9,8 & 4,6 & 0,55 & 17,8 & 17,3 & 3105 & 6,9 & 11,7 & 69 & 8861 & 2,85 & 2,2 & 5,0 & 126 \\
\hline & Elde & 162,88 & $2 * 1 \mathrm{WiB}$ & 17,7 & 5,0 & 0,70 & 25,2 & 20,7 & 7471 & 3,0 & 5,3 & 76 & 23172 & 3,10 & 1,7 & 4,0 & 141 \\
\hline & Eldekanal & 162,90 & $2 * 1 \mathrm{WiB}$ & 20,2 & 5,0 & 0,77 & 26,3 & 22,0 & 10098 & 2,6 & 4,7 & 80 & 32835 & 3,25 & 1,5 & 3,8 & 153 \\
\hline & Klosterbuschweg & 15,35 & $2 * 1 \mathrm{WiB}$ & 21,2 & 4,4 & 1,10 & 19,3 & 23,8 & 22723 & 3,4 & 5,3 & 55 & 54827 & 2,41 & 1,5 & 5,0 & 233 \\
\hline & Hackbuschstr. & 15,82 & $2 * 1 \mathrm{WiB}$ & 19,5 & 4,4 & 1,00 & 19,5 & 21,9 & 16226 & 3,6 & 5,3 & 49 & 36023 & 2,22 & 1,5 & 5,0 & 226 \\
\hline & Finkenkurger Weg & 16,35 & $2 * 1 \mathrm{WiB}$ & 19,5 & 4,4 & 1,00 & 19,5 & 21,4 & 16226 & 3,6 & 5,3 & 47 & 35234 & 2,17 & 1,5 & 5,0 & 226 \\
\hline & Straße 339 & 17,53 & $1 \mathrm{WiB}$ & 17,6 & 9,0 & 0,90 & 19,6 & 41,4 & 24647 & 3,9 & 5,3 & 35 & 45207 & 1,83 & 1,7 & 4,3 & 158 \\
\hline & Bredower Vorwerk & 29,69 & $1 \mathrm{WiB}$ & 8,6 & 10,2 & 0,44 & 19,5 & 31,0 & 2851 & 6,4 & 12,9 & 100 & 11425 & 4,01 & 2,3 & 4,3 & 87 \\
\hline & Bredower Str. & 30,70 & $1 \mathrm{WiB}$ & 12,6 & 8,7 & 0,60 & 21,0 & 32,7 & 7558 & 4,8 & 8,2 & 72 & 22484 & 2,97 & 2,0 & 5,0 & 148 \\
\hline & N. Paretzer ÖI & 31,70 & $1 \mathrm{WiB}$ & 8,0 & 8,8 & 0,44 & 18,1 & 28,6 & 2662 & 7,6 & 15,2 & 101 & 10711 & 4,02 & 2,3 & 3,2 & 37 \\
\hline & N. Paretzer $\mathrm{O} 2$ & 31,70 & I WiB & 14,9 & 8,8 & 0,72 & 20,6 & 36,0 & 12714 & 4,2 & 6,4 & 51 & 29030 & 2,28 & 1,9 & 3,5 & 88 \\
\hline & N. Paretzer $\mathrm{O} 3$ & 31,70 & I WiB & 8,4 & 8,8 & 0,49 & 17,0 & 29,8 & 3564 & 7,8 & 14,1 & 81 & 11687 & 3,28 & 2,3 & 3,5 & 51 \\
\hline & Hertefelder Str. B-HH & 36,02 & $2^{*} 1 \mathrm{WiB}$ & 13,5 & 4.9 & 0,72 & 18,7 & 19,4 & 7436 & 5,4 & 9,4 & 75 & 22728 & 3,06 & 2.0 & 4,0 & 104 \\
\hline & Hertefelder Str. HH-B & 36,02 & $2^{*} 1 \mathrm{WiB}$ & 13,5 & 4,5 & 0,72 & 18,7 & 20,5 & 7116 & 5,1 & 9,4 & 84 & 24051 & 3,38 & 2,0 & 4,0 & 104 \\
\hline
\end{tabular}

Alle untersuchten Brücken wurden als Einfeldträger mit Stützweiten zwischen $5,6 \mathrm{~m}$ und 24,6 $\mathrm{m}$ ausgeführt, wobei die zugehörigen Schlankheiten L/h von 14,4 bis maximal 26,3 reichen. Die mit Schotteroberbau ausgerüsteten Überbauten sind ausnahmslos schwimmend auf Elastomerlagern gelagert und ohne Schienenauszüge hergestellt. Die Abmessungen von Randkappe und Schutzschicht entsprechen jeweils den Regelausführungen der Deutschen Bahn (vgl. hierzu DB Richtzeichnungen in Modul 804.9010 der Ril 804 [8]). Auf Grundlage der Bauwerksdaten wurden die Massen pro laufenden Meter sowie die Biegesteifigkeiten der „nackten“ Brückenquerschnitte $\mathrm{El}_{\text {cal }}$ berechnet. Die Massen betragen bei eingleisigen Überbauten je nach Stützweite zwischen 15 und $28 \mathrm{t} / \mathrm{m}$, bei zweigleisigen Überbauten zwischen 30 und $50 \mathrm{t} / \mathrm{m}$. Die Steifigkeiten reichen durchschnittlich von etwa $5000 \mathrm{MNm}^{2}$ für Stützweiten kleiner $12,5 \mathrm{~m}$ bis 
zu Werten größer $30000 \mathrm{MNm}^{2}$ für Stützweiten von mehr als $20 \mathrm{~m}$, siehe Bild 3.1. Obwohl die zweigleisigen Überbauten etwa doppelt so breit sind wie die eingleisigen, bewegen sich ihre Steifigkeiten noch in der gleichen Größenordnung.

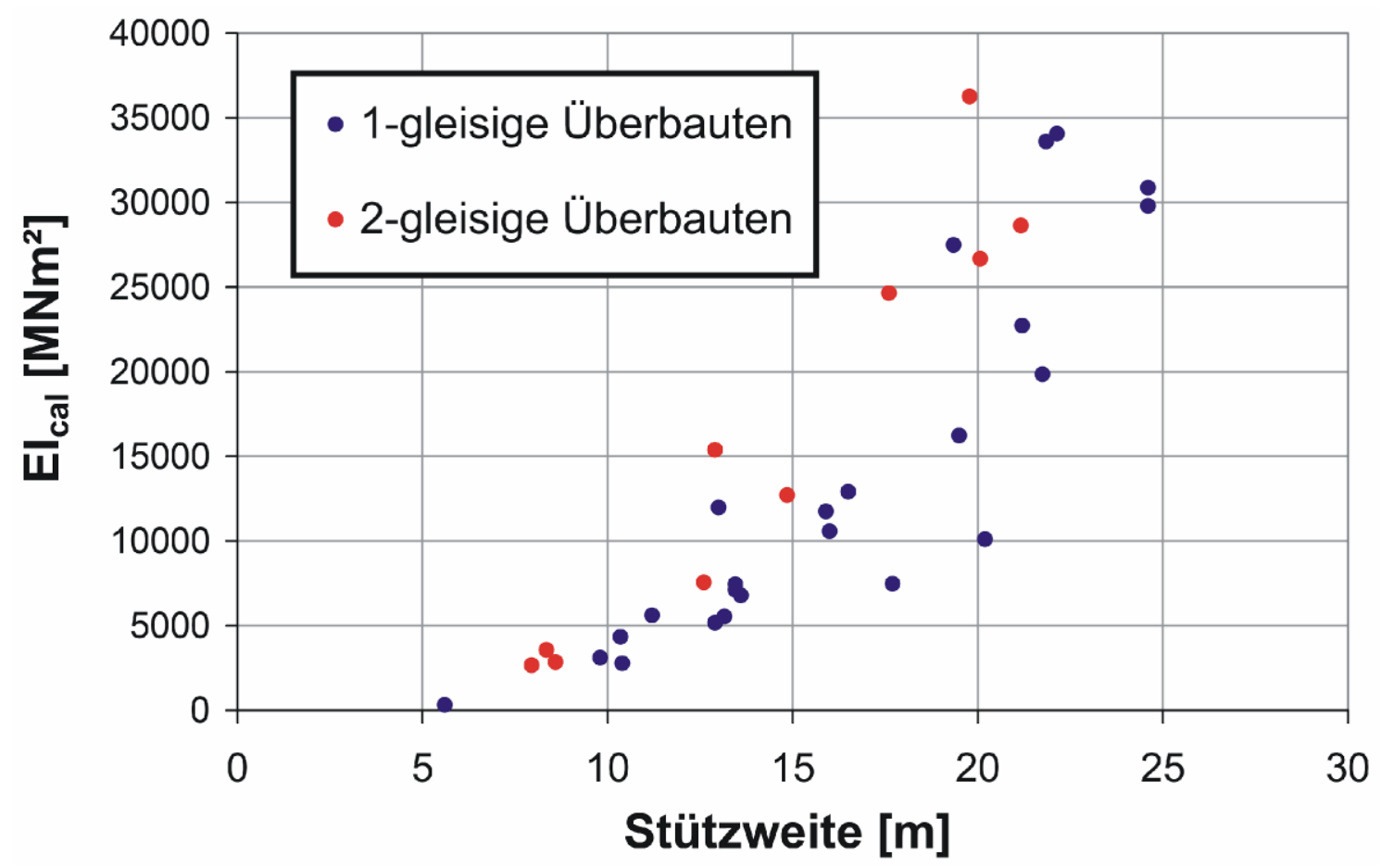

Bild 3.1: Rechnerische Biegesteifigkeit in Abhängigkeit von der Stützweite für ein- und zweigleisige Überbauten

Der Vergleich zwischen berechneter und gemessener erster Biegeeigenfrequenz $\left(\mathrm{n}_{0, \text { cal }}\right.$ bzw. $\left.\mathrm{n}_{0, \text { eff }}\right)$ zeigt, dass die Grundeigenfrequenz rechnerisch um mindestens $25 \%$ unterschätzt wurde. Insbesondere bei kurzen Stützweiten sind die Abweichungen erheblich (Bild 3.2a). So betragen die Unterschiede bei Brücken mit Stützweiten kleiner 12,5 m bis zu $100 \%$, im Extremfall sogar $110 \%$. Mit zunehmender Stützweite nehmen die Abweichungen deutlich ab und betragen noch etwa $25 \%$.

Aus den gemessenen Grundeigenfrequenzen lassen sich durch Umstellung von GI. (3) effektive "gemessene" Biegesteifigkeiten ableiten - unter Voraussetzung der entsprechenden Systemidealisierungen. Die effektive Steifigkeit $\mathrm{El}_{\text {eff }}$ beschreibt die tatsächlich im System wirkende Steifigkeit und kann folgendermaßen ausgedrückt werden (vgl. auch [57]):

$$
E I_{\text {eff }}=E I_{\text {cal }} \cdot\left(\frac{n_{0, \text { eff }}}{n_{0, c a l}}\right)^{2}
$$


Eine Gegenüberstellung der berechneten Biegesteifigkeiten $\mathrm{El}_{\text {cal }}$ mit den nach

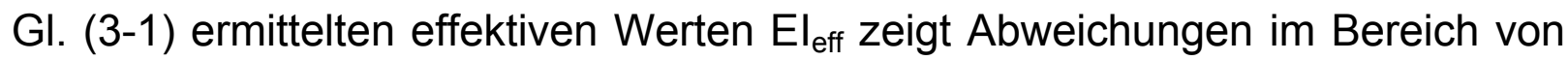
+60 bis $+450 \%$. Die tatsächlich vorhandenen Systemsteifigkeiten sind somit deutlich höher als die Steifigkeiten, die bei der herkömmlichen dynamischen Bemessung in Ansatz gebracht werden. Aufgrund der Abhängigkeit der effektiven Steifigkeit vom Verhältnis der gemessenen zur berechneten Grundfrequenz macht sich der Effekt wiederum besonders bei kurzen Überbauten bemerkbar (Bild 3.2b). Signifikante Unterschiede bezüglich zusätzlicher Steifigkeitsbeiträge ein- und zweigleisiger Überbauten sind nicht zu erkennen.

a)

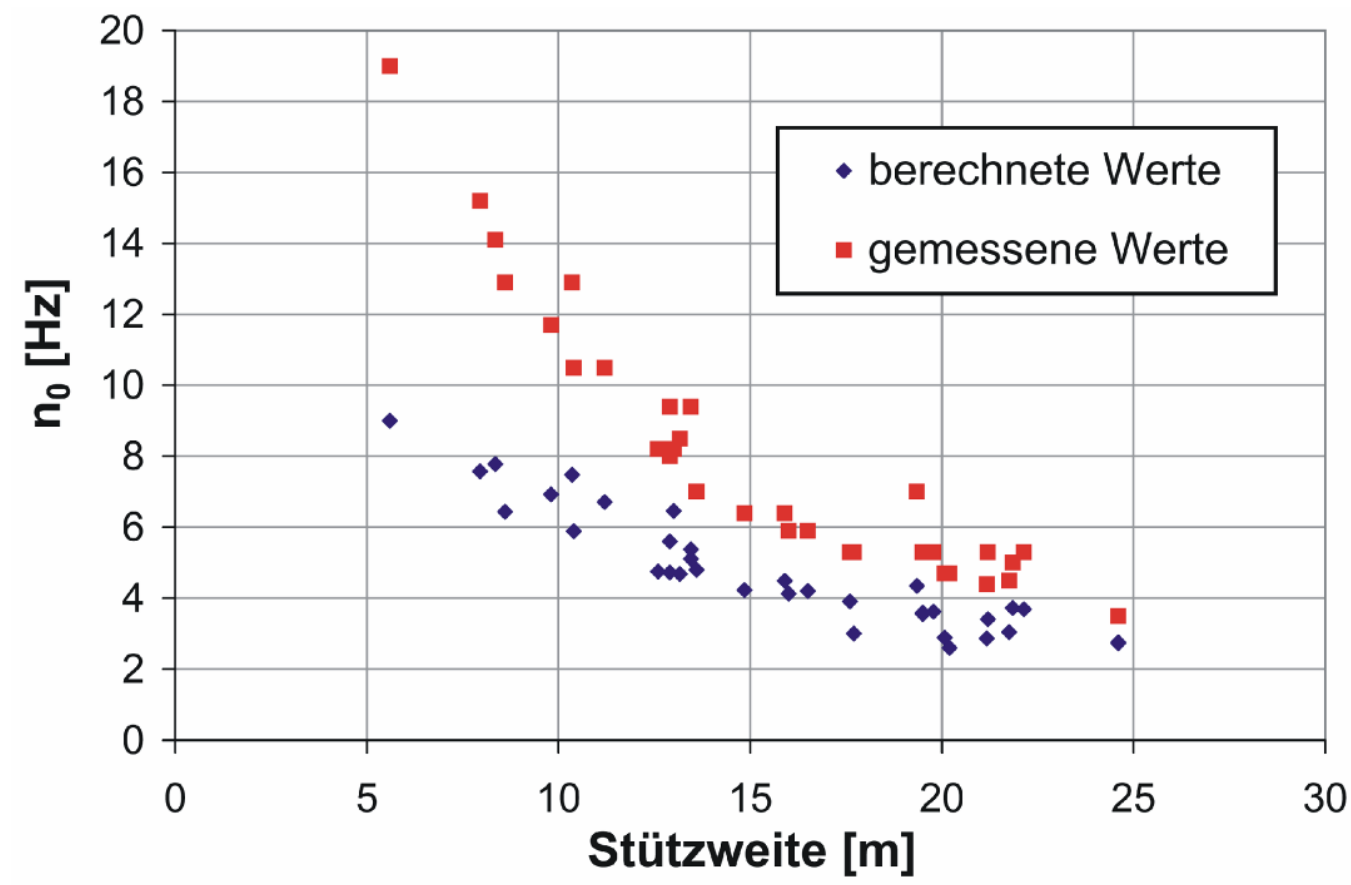

b)

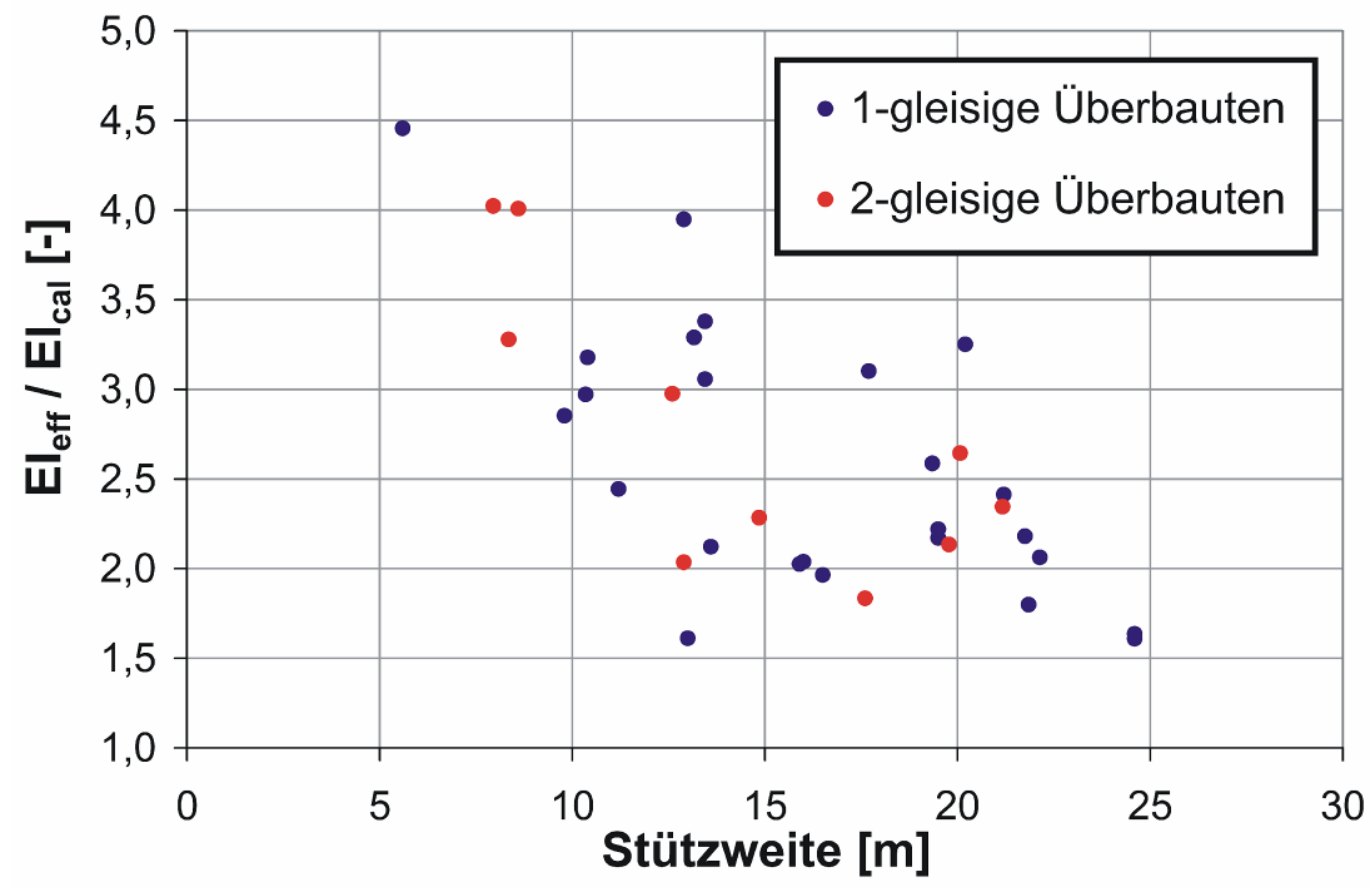


c)

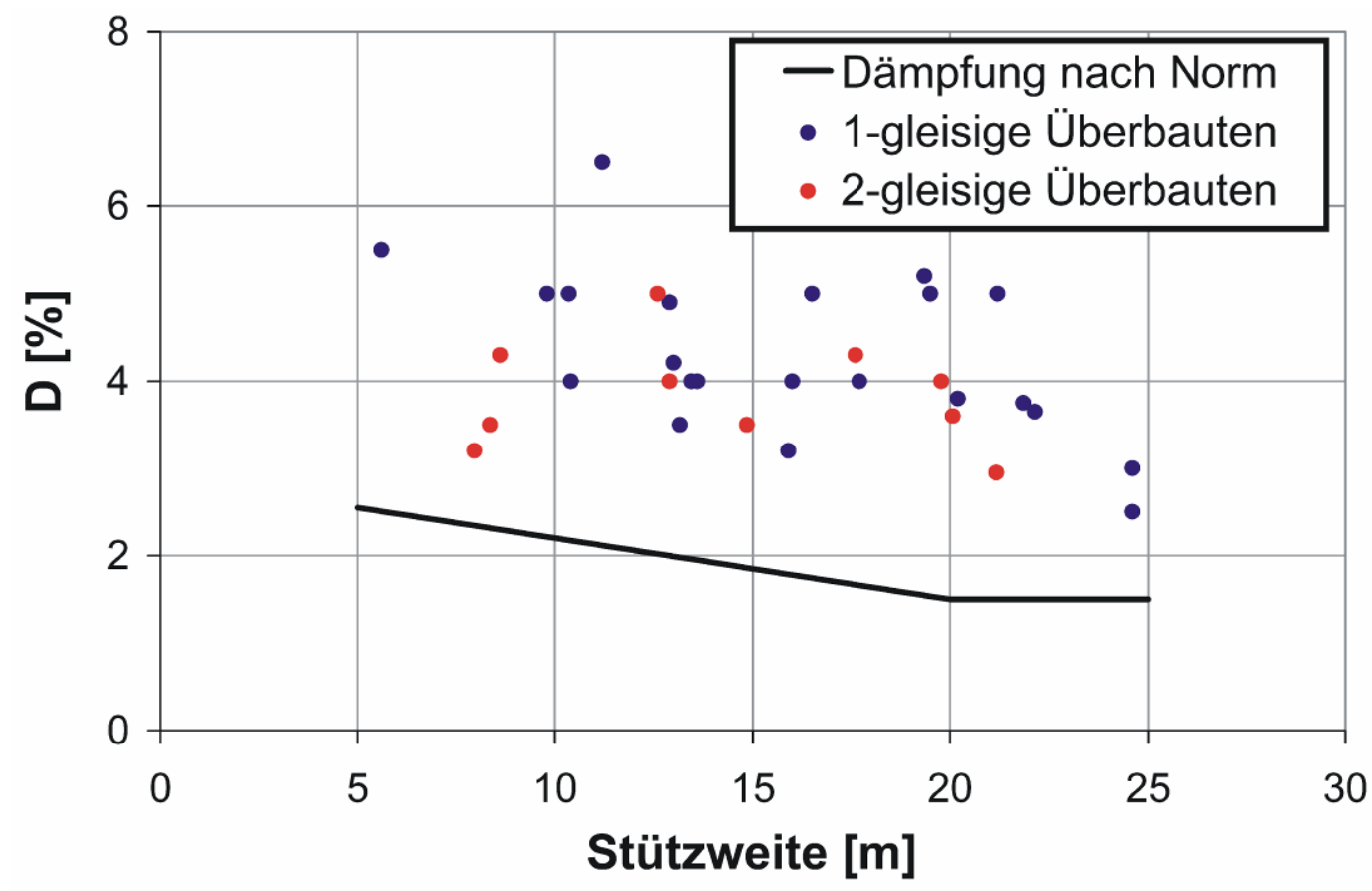

Bild 3.2: Aufgetragen in Abhängigkeit von der Stützweite: a) Berechnete und gemessene erste Eigenfrequenzen; b) Quotienten aus effektiven „gemessenen“ und berechneten Steifigkeiten; c) nach Norm anzusetzende und gemessene Dämpfungswerte (Lehrsche Dämpfung)

Bei der Auswertung der Dämpfungseigenschaften zeigt sich, dass die gemessenen Werte $\left(D_{\text {eff }}\right)$ um den Faktor 1,35 bis 2,35 größer sind als die nach Norm anzusetzenden Dämpfungswerte $\left(D_{\text {cal }}\right)$. In Tabelle 3.1 sind jeweils die Lehr'schen Dämpfungswerte angegeben. Die normativen Dämpfungsvorgaben liegen also bei allen untersuchten WIB-Überbauten deutlich auf der sicheren Seite (Bild 3.2c). Eine eindeutige Abhängigkeit von der Stützweite, wie bei den Steifigkeitsunterschieden, ist bei den Dämpfungseigenschaften nicht zu erkennen. Die Verteilung der Dämpfungswerte über der Stützweite ist durch eine starke Streuung gekennzeichnet. Eine Regelmäßigkeit zeigt sich ansatzweise darin, dass die Dämpfungswerte zweigleisiger Überbauten im Vergleich zu eingleisigen Überbauten (mit durchgängiger Schotterschicht) tendenziell etwas niedriger sind. Die Datenmenge reicht allerdings nicht aus, um allgemeingültige Schlussfolgerungen abzuleiten.

Im Vorfeld der Auswertung wurde vom Autor vermutet, dass zusätzliche bauliche Elemente wie z.B. Schallschutzwände einen signifikanten Einfluss auf die Systemdämpfung haben würden. Diese Vermutung konnte durch die Messergebnisse nicht bestätigt werden. Obwohl auf einigen der untersuchten Brücken ein- oder sogar beidseitig Schallschutzwände vorhanden waren, wurde keine 
einheitliche Tendenz hinsichtlich einer Dämpfungserhöhung festgestellt. In einigen Fällen ergaben sich sogar kleinere Dämpfungswerte als bei Überbauten ohne Schallschutzwände.

An allen Überbauten wurden Messungen bei schnee- und frostfreier Witterung durchgeführt (im Zeitraum April bis Oktober). Den Empfehlungen in [33], bemessungsrelevante Messkampagnen an Eisenbahnbrücken mit Schotteroberbau nicht im Winter durchzuführen, wurde somit entsprochen. Interessanterweise wurden an den Brücken „EÜ Havel“ und „EÜ Steinförder Str.“ Messungen sowohl im Winter (20./21. Februar 2001) als auch im Sommer (17./18. Juli 2001) vorgenommen. Die Temperaturen betrugen während der ersten Messung um die Null Grad Celsius (Angaben Deutscher Wetterdienst). Trotz der niedrigen Temperaturen wurden im Vergleich zu den Messungen im Sommer allerdings keine Unterschiede der Messergebnisse festgestellt.

\subsection{Bauwerks-Monitoring an der WIB-Brücke EÜ Erft- talstraße}

\subsubsection{Hintergrund}

Im Rahmen der Messkampagnen, die wie unter 3.1.1 beschrieben von der Deutschen Bahn durchgeführt wurden, wurde die WIB-Brücke EÜ Erfttalstraße bei Kerpen (ABS 4 Köln - Aachen, km 21,223) gleich zweimal messtechnisch untersucht. Die Brücke wurde im Jahre 2001 als Teil der HGV-Neubaustrecke Köln-Aachen gebaut, die mittlerweile weiterreicht bis Brüssel und Paris.

Die Strecke sollte sowohl von ICE-3 als auch von Thalys-Zügen genutzt werden. Allerdings ließ eine rechnerische Biegeeigenfrequenz von $2,8 \mathrm{~Hz}$ auf eine Resonanzgefährdung für den ICE-3- und Thalys-Verkehr schließen. Aus diesem Grund fanden im September 2002 die ersten Messungen statt, die Aufschluss über Grundeigenfrequenz und Dämpfung geben sollten. Zur Bestimmung der dynamischen Parameter wurden Abklingkurven nach Zugüberfahrten ausgewertet.

Mit einer gemessenen 1. Biegeeigenfrequenz von $3,5 \mathrm{~Hz}$ und einer Lehr'schen Dämpfung von 2,5\% [6] wurden im Vergleich zu anderen untersuchten WIBÜberbauten relativ geringe Differenzen zwischen Rechen- und Messwerten der Grundeigenfrequenz und Dämpfung festgestellt. Außerdem zeigten die durchgeführten Messungen nach dem Freifahren der Überbauten durch das jeweils letzte Drehgestell ein sehr "weiches“ und intensives Schwingverhalten.

Zur weiteren Qualifizierung des dynamischen Verhaltens wurden daher im Juni 2003 weitere begleitende Messungen während der Abnahmefahrten mit hohen 
Geschwindigkeiten durchgeführt. Auf Grundlage dieser Messungen konnte nachgewiesen werden, dass die Brücke durch ICE-3- und Thalys-Züge zwar nicht mit der ursprünglich geforderten Geschwindigkeit von $v_{\max }=300 \mathrm{~km} / \mathrm{h}$, jedoch mit $v_{\max }=250 \mathrm{~km} / \mathrm{h}$ befahren werden kann, ohne dass es zu bemessungsrelevanten Resonanzeffekten kommt [6], vgl. hierzu auch Abschnitt 7.

Um den Ursachen für das problematische dynamische Verhalten der Erfttalbrücke genauer nachzugehen, wurde entschieden, im Rahmen des Forschungsprojektes DETAILS [52] weiterführende Messungen am Bauwerk vorzunehmen. Zunächst wurde im April 2007 eine Messkampagne durchgeführt, die der Systemidentifikation diente, d. h. der detaillierten Bestimmung der dynamischer Kennwerte Eigenfrequenzen, Eigenformen und Dämpfungswerte. Dabei wurden mit sehr empfindlichen Beschleunigungssensoren ambiente Schwingungen der Struktur gemessen. Die Kampagne bildete die Grundlage für die Generierung eines komplexen numerischen Modells, das mit Hilfe von "model-updating“Techniken optimiert wurde. Einzelheiten hierzu sind [50] zu entnehmen.

Um außerdem das Verhalten unter Fahrzeuglasten genauer zu untersuchen, wurde im nächsten Schritt ein dauerhaftes Monitoringsystem an der Brücke installiert. Die Installation fand im November 2008 statt. Das System ist derzeit noch in Betrieb. Im Folgenden wird das Langzeitmonitoring näher erläutert. Anschließend werden Ergebnisse präsentiert, die in Bezug auf die vorliegende Arbeit von Interesse sind.

\subsubsection{Bauliche Durchbildung und Messsystem}

Es handelt sich bei der WIB-Brücke EÜ Erfttalstraße um zwei baulich getrennte eingleisige Überbauten mit durchgehendem Schotterbett, siehe Bild 3.3 und Bild 3.4. Sie wurde direkt neben einer bestehenden Stahlbrücke errichtet, auf der regionale S-Bahnen verkehren.

Die Spannweiten der beiden Brückendecks betragen 24,6 m, die Überbaubreiten jeweils etwa $5,25 \mathrm{~m}$. Beide Überbauten bestehen aus jeweils elf einbetonierten HEM 1000 S235 Standard-Walzprofilen. Unter Berücksichtigung einer Betondeckung von $8 \mathrm{~cm}$ oberhalb der Stahlträger ergibt sich daraus eine Höhe der WIB-Platten von etwa 1,10 m. Die Lagerung der Überbauten erfolgt punktförmig auf jeweils vier Elastomerlagern ohne Verformungsbehinderung in Längsrichtung (schwimmende Lagerung). 
a)

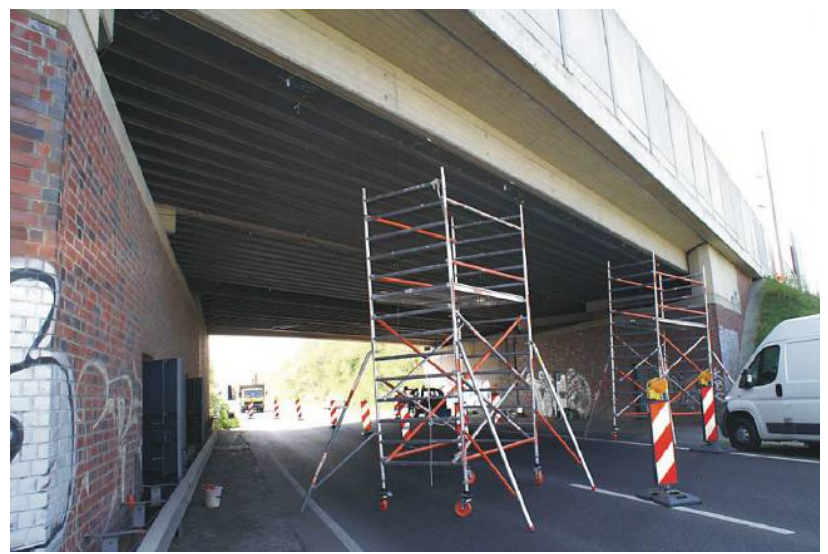

b)

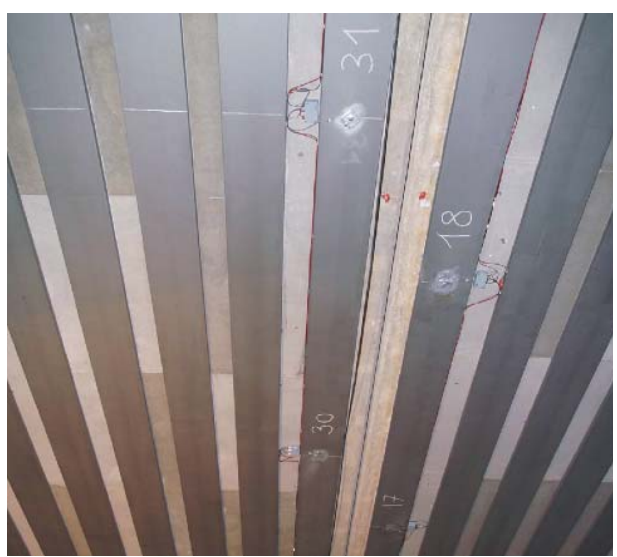

c)

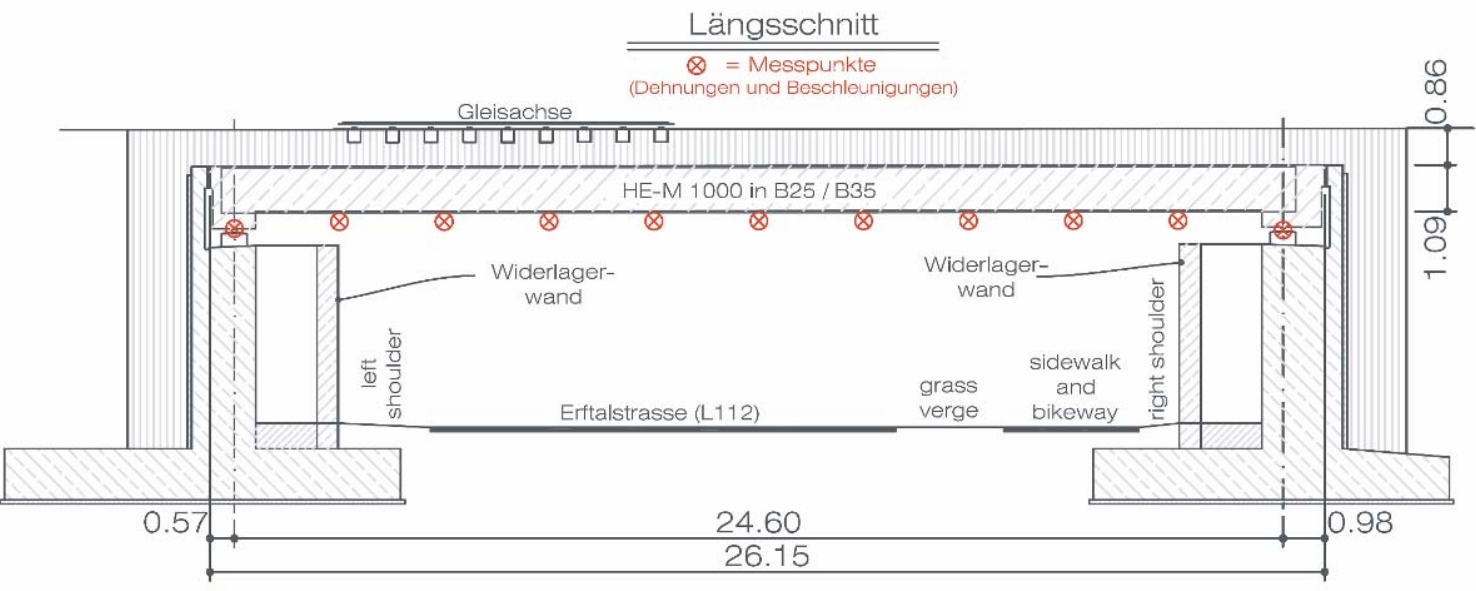

d)

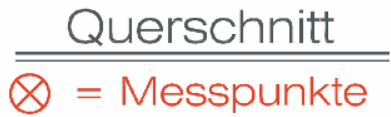

(Dehnungen und Beschleunigungen)

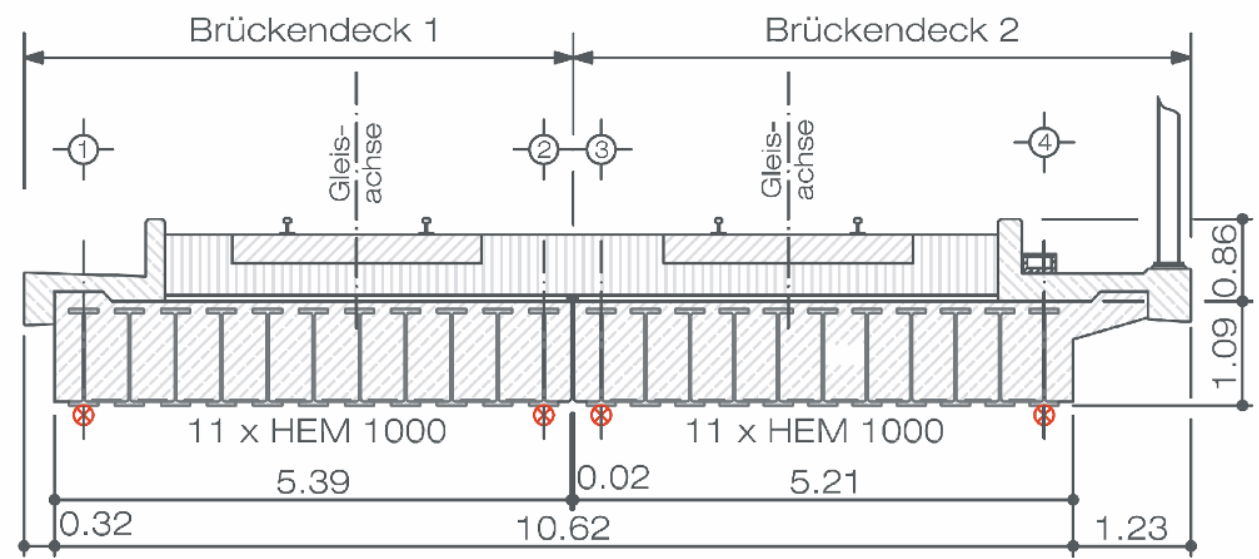

Bild 3.3: a) Installation des Monitoringsystems an der WIB-Brücke EÜ Erfttalstraße b) Untersicht auf die Messachsen zwei und drei c) Längsschnitt d) Brückenquerschnitt (Positionen der Messsensoren jeweils in rot markiert) 
Insgesamt wurden die beiden Überbauten mit 44 Messsensoren bestückt (22 je Überbau), wobei je elf Sensoren mit einem einheitlichen Abstand von 2,46 m am Unterflansch der jeweils äußeren Stahlträger appliziert wurden, siehe Bild 3.4. Mit jedem Messsensor werden Stahldehnungen und Beschleunigungen in vertikaler Richtung gemessen. Nur in den Auflagerachsen wird auf eine Dehnungsmessung an den Stahlbeton-Querträgern verzichtet. Es wird davon ausgegangen, dass die Dehnungen hier vernachlässigbar klein sind.

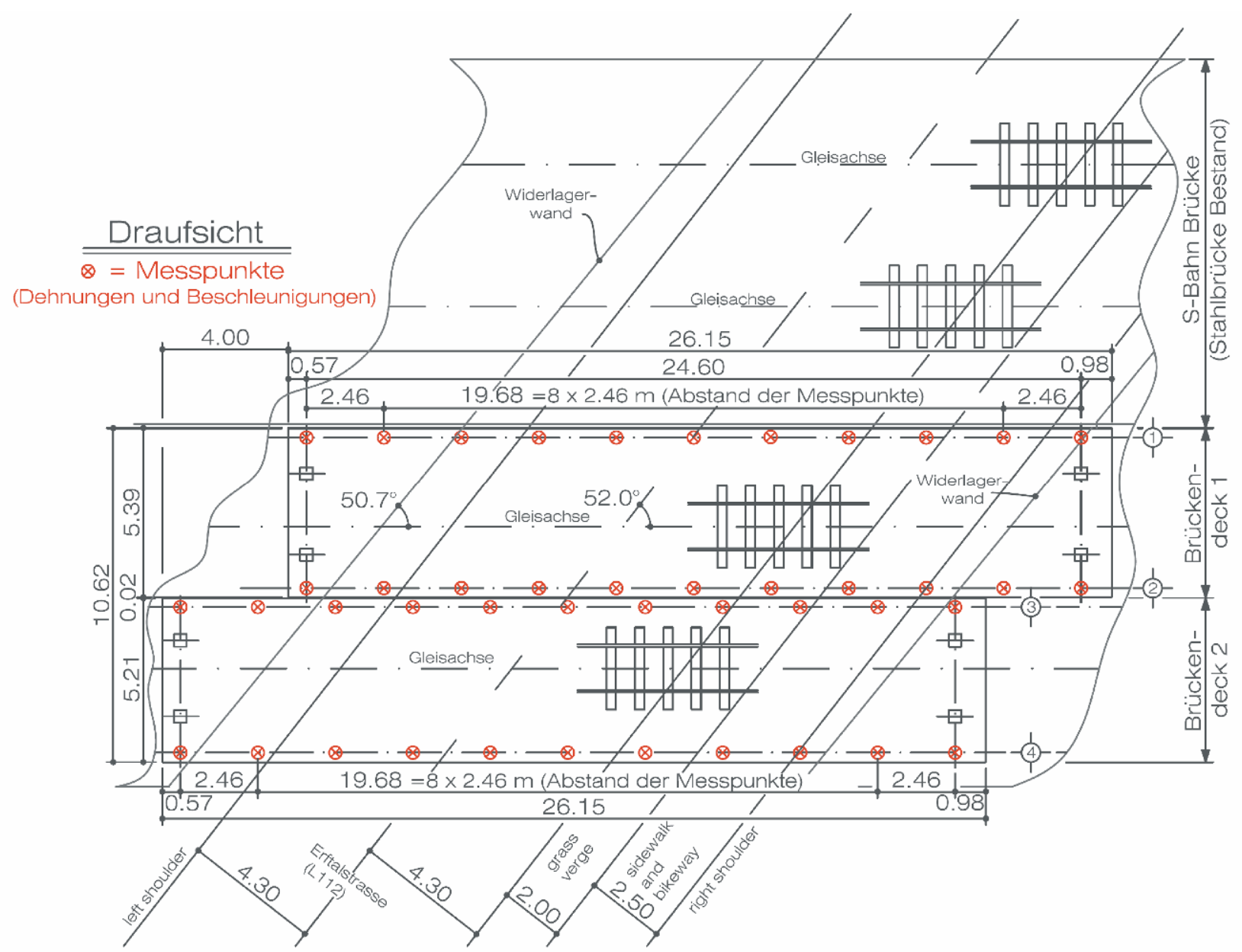

Bild 3.4: Draufsicht auf die messtechnisch untersuchte Brücke mit vier Messachsen (Positionen der Messsensoren in rot markiert)

Der Auswahl der Messsensoren lag eine Kosten-Nutzen-Analyse zugrunde, vgl. Bild 3.5. Mit qualitativ hochwertigen Sensoren, d.h. Sensoren mit einer hohen Empfindlichkeit bei gleichzeitig geringem Hintergrundrauschen, lassen sich ambiente Schwingungen mit sehr kleinen Amplituden aufzeichnen. Entsprechende Messdaten werden beispielsweise bei der Durchführung von „HealthMonitoring"-Projekten benötigt, vgl. [60]. Eine ähnliche Zielsetzung wurde im Rahmen der bereits erwähnten Messkampagne an der Erfttalbrücke zur Systemidentifikation verfolgt, siehe [50]. 
Die Zielsetzung beim Langzeitmonitoring war hingegen eine andere. Hier sollte das dynamische Systemverhalten unter Zuglasten untersucht werden, das durch relativ hohe Schwingungsamplituden gekennzeichnet ist. Entsprechende Messsignale lassen sich auch mit weniger hochwertigen Sensoren erfassen, die deutlich günstiger als die oben erwähnten hochsensiblen Sensoren sind.

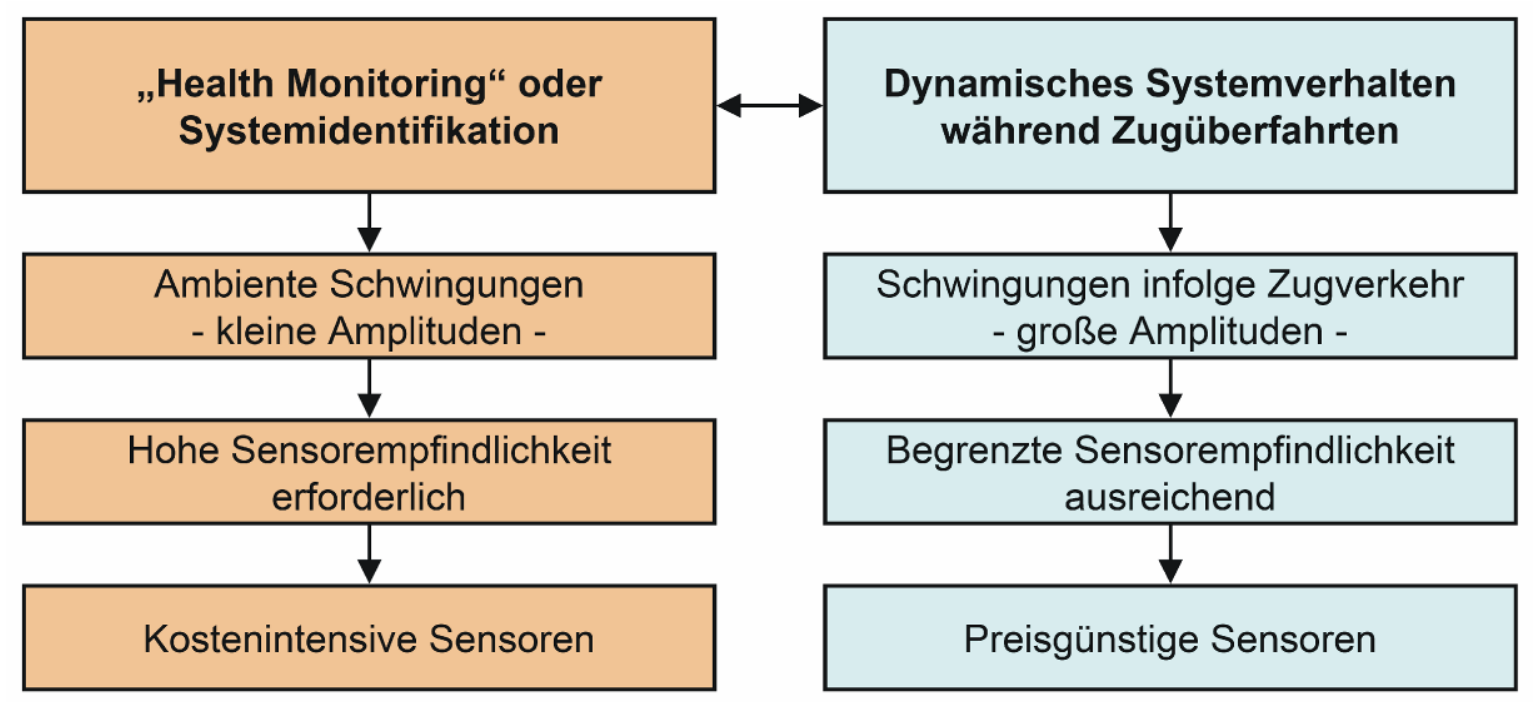

\section{Bild 3.5: Unterscheidung unterschiedlicher Monitoring-Zielsetzungen mit den sich daraus ergebenen Konsequenzen für die Wahl der Sensoren}

Im Rahmen des Forschungsprojektes DETAILS stand ein bestimmtes Budget für die Installation eines Monitoringsystems zur Verfügung. Unter Verwendung der preisgünstigen Sensoren (Hersteller GBF mbH, Aachen), konnte die ungewöhnlich große Anzahl von 44 Messpunkten realisiert werden, die insbesondere im Hinblick auf die in Abschnitt 3.2.5 thematisierte Berechnung von Systemverformungen von Bedeutung ist. Charakteristische Kennwerte des installierten Messsystems sind in Tabelle 3.2 gegeben.

Das System wird mit einer Abtastrate von $100 \mathrm{~Hz}$ betrieben, welche die Erfassung aller relevanten Frequenzanteile während Zugüberfahrten erlaubt. Auf dem Messrechner vor Ort werden kontinuierlich alle Messsignale temporär aufgezeichnet. Mittels Trigger-Funktion werden anschließend die Überfahrtsereignisse selektiert, via UMTS auf einen externen Server übertragen und dort dauerhaft gesichert. Die nach Datum und Uhrzeit sortierten Überfahrtzeitschriebe können dann nach Bedarf vom Datenserver abgerufen werden. 
Tabelle 3.2: Monitoring Erfttalbrücke: Kennwerte Messsystem

\begin{tabular}{ll}
\hline Monitoring System & \\
\hline Anzahl Sensoren & 44 \\
Messgrößen je Sensor & ACC vertikal, Dehnungen \\
Bandbreite & $2,5 \mathrm{Mbit}$ \\
Abtastrate einzelner Sensor & $10 \mathrm{kSps}$ \\
Maximale Abtastrate Gesamtsystem & $200 \mathrm{~Hz}$ bei 160 Kanälen \\
Schutz & IP 66 \\
Speicherkapazität lokal & $1 \mathrm{~TB}$ \\
Externer Datentransfer & via UMTS \\
Datenstruktur & ASCII-Zeitreihen \\
Speicherkapazität Archiv & externer Server >> 1TB \\
\hline Qualität Dehnungssignal & \\
\hline SINAD & $70 \mathrm{~dB}$ \\
THD & $65 \mathrm{~dB}$ \\
Maximal messbares Signal & über Software einstellbar, max. Dehnung $2 \%$ \\
Auflösung & $24 \mathrm{Bit}$ \\
Dynamische Bandbreite & $140 \mathrm{~dB}$ \\
Rauschen & $0,25 \mathrm{ppm} \mathrm{0,1-20} \mathrm{Hz}$ \\
\hline Qualität Beschleunigungssignal & \\
\hline SINAD & $70 \mathrm{~dB}$ \\
THD & $65 \mathrm{~dB}$ \\
Maximal messbares Signal & über Software einstellbar $\pm 2 \mathrm{~g} / \pm 6 \mathrm{~g}$ \\
Auflösung & $24 \mathrm{Bit}$ \\
Dynamische Bandbreite & $140 \mathrm{~dB}$ \\
Rauschdichte & $12 \mu \mathrm{g} / \mathrm{NHz}$ \\
\hline
\end{tabular}

\subsubsection{Allgemeines zur Messauswertung}

Die Dehnungs- und Beschleunigungszeitschriebe aus den gemessenen Überfahrtereignissen liegen im ASCII-Format vor. Dies ermöglicht einen unkomplizierten Zugriff durch geeignete Softwareapplikationen zur Datenauswertung. Aufgrund der enormen Datenmenge - pro Tag werden etwa 100 bis 200 Zugüberfahrten erfasst - wurde mit dem Software-Tool FOX [19] eine automatisierte Auswertung in MATLAB [34] programmiert.

Das Programm FOX beinhaltet die folgenden Routinen, die bei Auswertung der einzelnen Zugüberfahrten ausgeführt werden:

- Zugtyperkennung und Bestimmung der Überfahrtgeschwindigkeiten (siehe Abschnitt 3.2.4) 
- Bestimmung der maximalen Bauwerksreaktionen: Dehnungen, Beschleunigungen, Durchbiegungen (zu deren Berechnung siehe Abschnitt 3.2.5)

- Bestimmung von Systemdämpfung und Grundeigenfrequenz aus der Abklingkurve nach Beendigung der Überfahrt, siehe Abschnitt 3.2.7

Im Vorlauf zu diesen Auswerteroutinen werden die Messsignale tiefpassgefiltert, um nicht-relevante hochfrequente Schwingungskomponenten zu eliminieren. Dabei wurde in Anlehnung an die Anforderungen des DIN-Fachbericht 101 [11] bezüglich der zu berücksichtigenden Frequenzanteile beim Nachweis der maximal zulässigen Beschleunigungen eine Grenzfrequenz von $20 \mathrm{~Hz}$ festgelegt.

Alle für die Auswertung relevanten Parameter werden in einer Konfigurationsdatei definiert. Die Ergebnisausgabe erfolgt tabellarisch. Für jede Zugüberfahrt werden die Ergebnisse in einer Zeile zusammengefasst. Kann eine der Auswerteroutinen nicht fehlerfrei ausgeführt werden, so erfolgt in der entsprechenden Spalte der Ausgabetabelle eine Fehlermeldung (mögliche Fehlerursachen z. B.: Zugtyp nicht identifizierbar, Ausschwingkurve für Auswertung von Eigenfrequenz und Dämpfung ungeeignet).

Wie in den folgenden Abschnitten deutlich wird, ist es im Rahmen der Messdatenauswertung wichtig, die Zeitpunkte, zu denen Maximalwerte in den Zeitschrieben auftreten, möglichst exakt zu bestimmen. Hiervon hängt die Genauigkeit ab, mit der Überfahrtgeschwindigkeit, Eigenfrequenz und Dämpfungswerte bestimmt werden.

Die Abtastrate der gemessenen Zeitreihen kann daher vor Beginn der MATLABAuswerteroutinen erhöht werden. Entsprechend der Vorgabe in der Konfigurationsdatei können dazu beliebig viele diskrete Zeitpunkte jeweils zwischen den einzelnen Messwerten eingefügt werden. Die zugehörigen Ordinaten werden unter Verwendung einer polynomischen Spline-Funktion bestimmt. Hierzu wird eine standardmäßige MATLAB-Routine eingesetzt. Die gemessenen Werte bleiben bei diesem Vorgang unverändert.

\subsubsection{Zugtypen und Überfahrtgeschwindigkeiten}

Auf der HGV-Strecke Köln-Düren verkehren planmäßig nur wenige Zugtypen. Mit Ausnahme von sehr selten verkehrenden internationalen Reisezügen handelt es sich hierbei um ICE-3-Züge (ICE-1 und -2-Züge können die Strecke nicht befahren, weil sie nicht mehrstromfähig sind), Thalys-, Regional-Express- sowie Güterzüge. In Bild 3.6 sind Dehnungs- und Beschleunigungszeitverläufe dieser Zugtypen dargestellt. Sie wurden jeweils in Feldmitte gemessen. 
Die Zeitschriebe der einzelnen Zugtypen weisen charakteristische Merkmale auf, die eine automatisierte Zuordnung zum jeweiligen Zugtyp ermöglichen. Beispielsweise besteht der auf der Strecke Köln-Aachen verkehrende ICE-3-Zug aus acht Wagen und verfügt damit über insgesamt neun Achsgruppen (vgl. Bild 2.4 - Darstellung von zwei Achsgruppen mit je vier Achsen). Jede dieser neun Achsgruppen verursacht ein Maximum im Dehnungszeitschrieb. Anschließend erfolgt das freie Ausschwingen, siehe Bild 3.6a. Im Vergleich dazu besitzt der Thalys-Zug ein Doppelmaximum zu Beginn der Überfahrt und insgesamt elf Kurvenmaxima, vgl. Bild 3.6b. Der durch RE-Züge verursachte Dehnungsverlauf mit fünf ausgeprägten und einem kleinen Dehnungsmaximum ist gleichfalls charakteristisch und erlaubt eine automatisierte Erkennung durch das Auswerteprogramm FOX. Hier muss allerdings unterschieden werden, ob sich die Antriebslok vorne oder hinten befindet - erkennbar an einem ausgeprägten Maximum an entsprechender Stelle. Die in Bild 3.6c dargestellten Zeitschriebe stammen von einem RE-Zug mit vorne befindlicher Lok. Güterzüge sind durch ihre Länge und durch eine deutliche Unregelmäßigkeit in den Messschrieben gekennzeichnet, vgl. Bild 3.6d.

a)

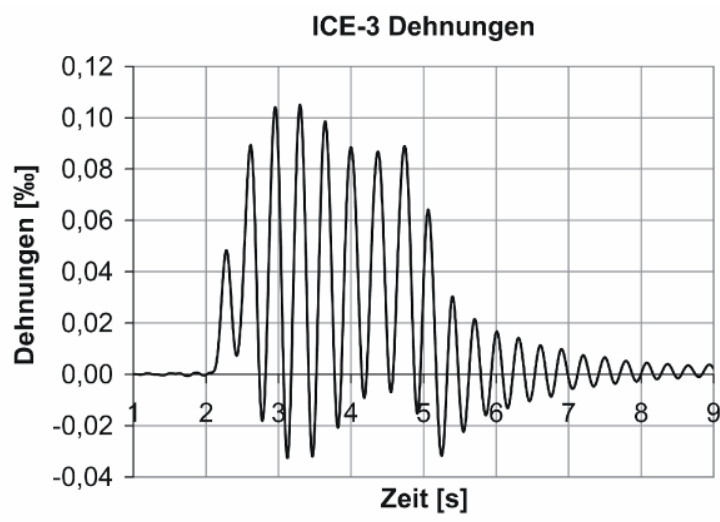

b)

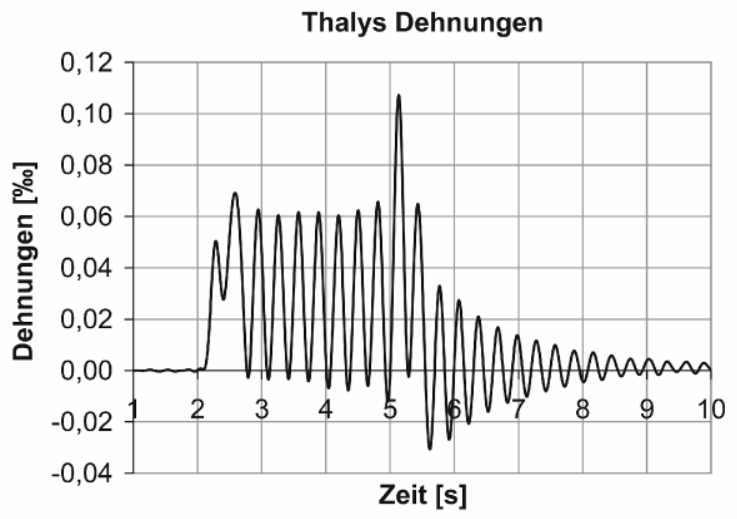

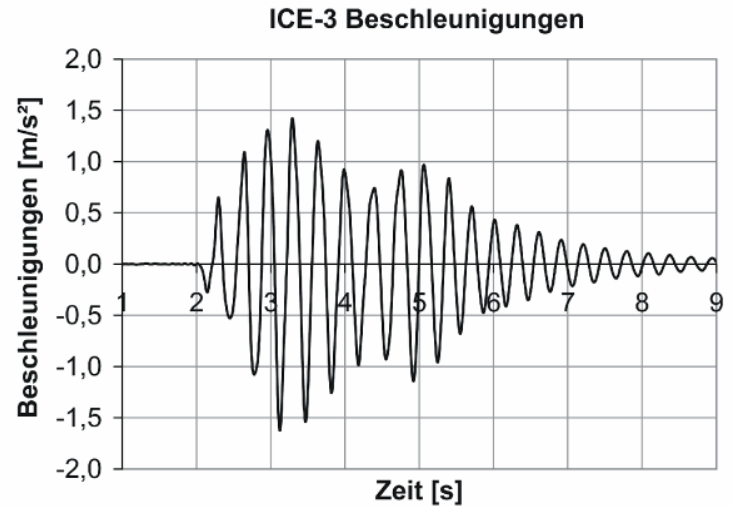

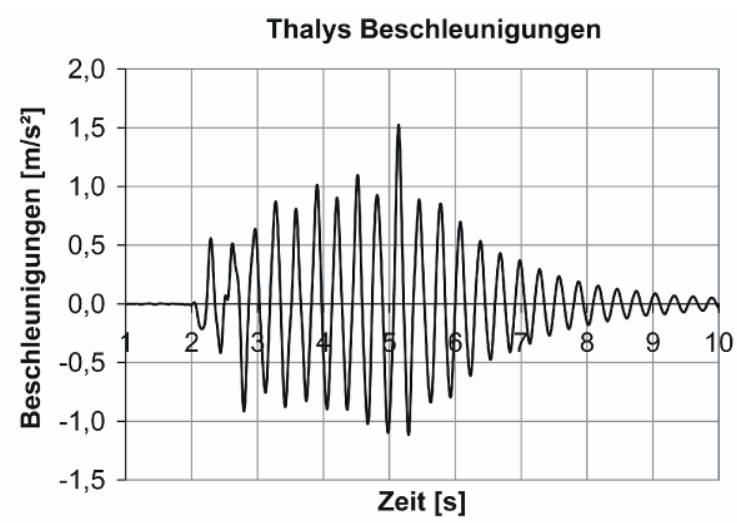


c)

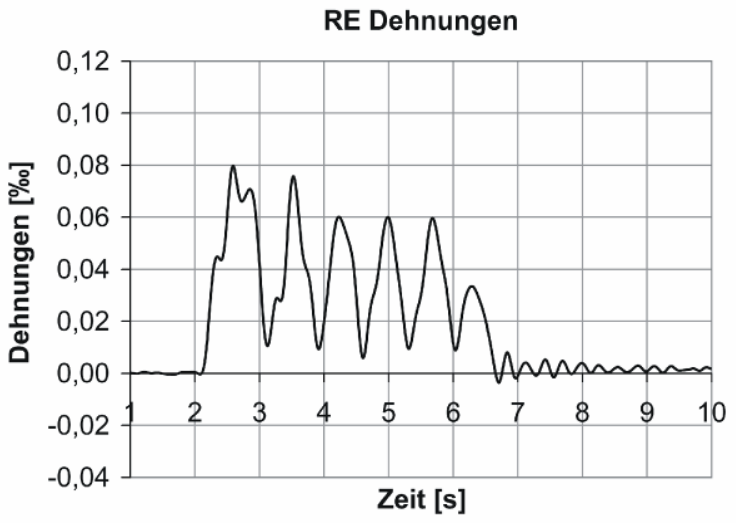

d)

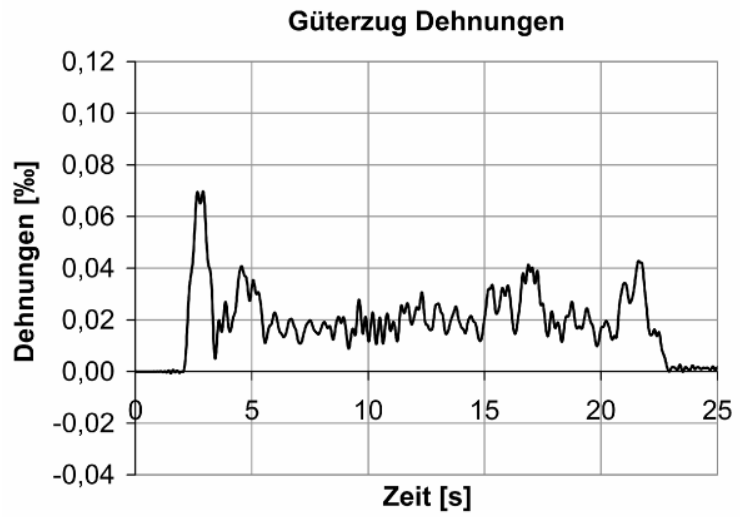

RE Beschleunigungen
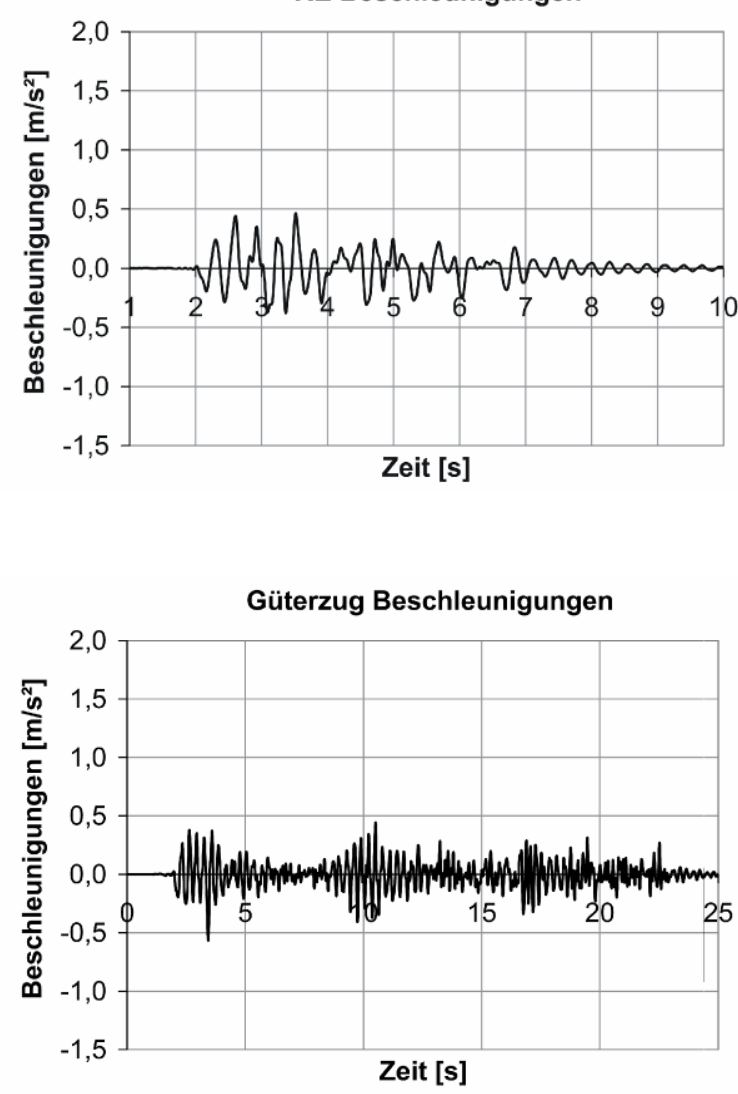

Bild 3.6: Dehnungs- und Beschleunigungsmessungen während Zugüberfahrten in Feldmitte a) ICE-3 b) Thalys c) RE-Express d) Güterzug

Die Kriterien, die bei der Zugtyperkennung zum Tragen kommen, lassen sich folgendermaßen zusammenfassen:

- Gesamtanzahl der Maxima

- Absolute Größe der Maxima

- Größe der Maxima relativ zueinander

- Auftreten von Doppelmaxima

Darüber hinaus bietet der Streckenfahrplan der Deutschen Bahn die Möglichkeit zu kontrollieren, welcher Zugtyp zu einer bestimmten Zeit auf der Brücke verkehrt.

Bei Kenntnis des Zugtyps kann die Überfahrtgeschwindigkeit bestimmt werden. Die Länge über Puffer $L_{\text {üp }}$ von ICE, Thalys und RE-Express sind jeweils einheitlich (entsprechende Werte sind in Bild 2.4 gegeben). Indem aus dem jeweiligen Dehnungszeitschrieb die Dauer $\Delta \mathrm{t}$ ermittelt wird, die benötigt wird, um die Stre- 
cke $L_{u ̈ P}$ zurückzulegen, kann die Überfahrtgeschwindigkeit wie folgt berechnet werden:

$$
v_{\text {cross }}=\frac{L_{\ddot{u} P}}{\Delta t}
$$

Um $\Delta t$ und damit auch $v_{\text {cross }}$ möglichst exakt zu bestimmen, wird eine Interpolation der Messwerte, wie in Abschnitt 3.2.3 beschrieben, empfohlen.

Bei Güterzügen ist die Geschwindigkeitsbestimmung so nicht möglich. Die gemessenen Dehnungsmaxima können zum einen nicht eindeutig den einzelnen Achsgruppen zugeordnet werden, außerdem sind die Wagenlängen bzw. Werte für $L_{u ̈ P}$ uneinheitlich.

Um das Verfahren zur Bestimmung der Überfahrtgeschwindigkeiten zu verifizieren, wurden mit einer Radarpistole Zuggeschwindigkeiten vor Ort gemessen und mit den Rechenwerten abgeglichen. Die Unterschiede zwischen den jeweils ermittelten Geschwindigkeiten betrugen weniger als $5 \%$.

ICE-3 Dehnungen

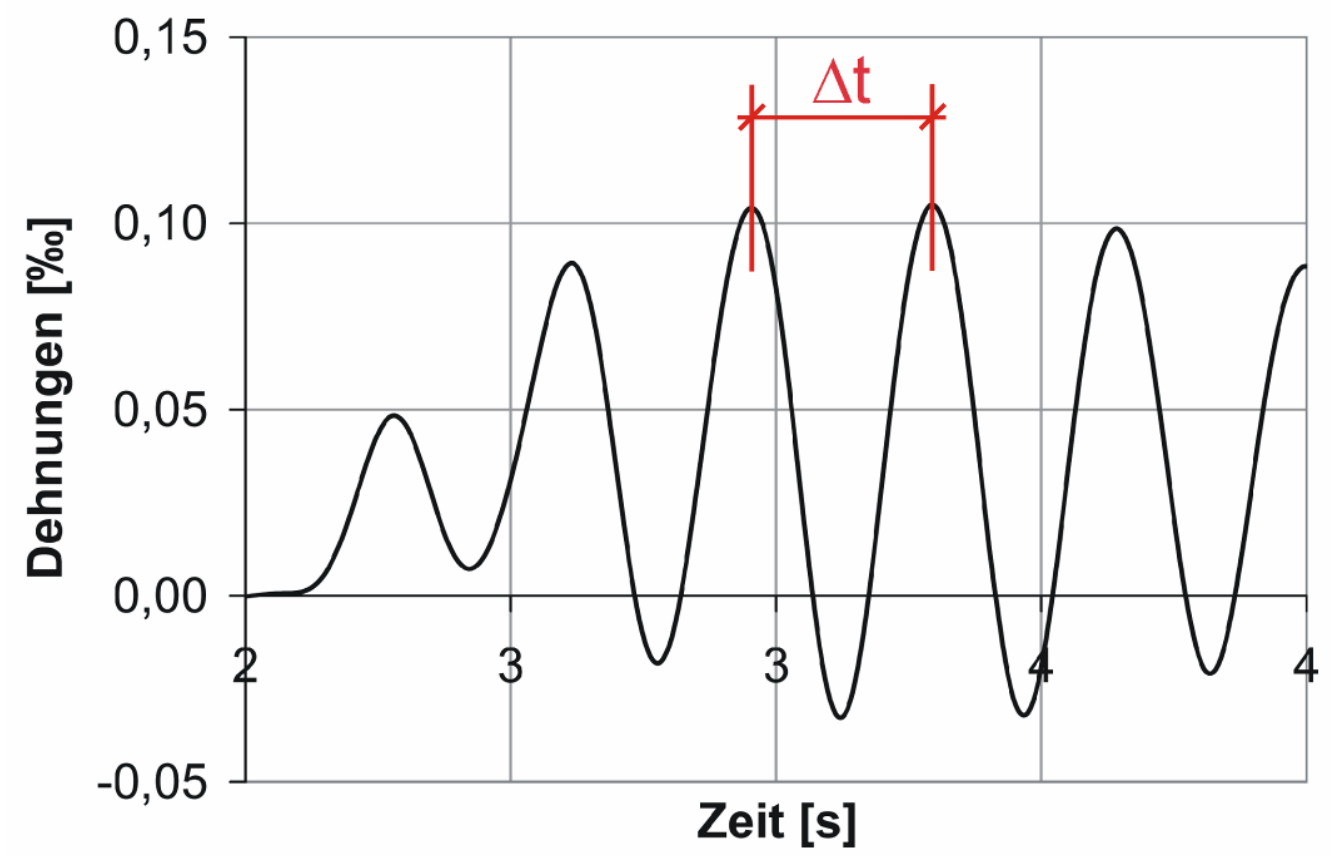

\section{Bild 3.7: Zur Bestimmung der Überfahrtgeschwindigkeiten}

\subsubsection{Berechnung der Brückendurchbiegungen}

In [48] und [49] wurde ein matrizielles Verfahren entwickelt, um aus den gemessenen Dehnungen die Durchbiegungen der Überbauten zu berechnen, die sich während Zugüberfahrten einstellen. Dazu wird jede der vier Messachsen (vgl. Bild 3.4a) in „Einzelelemente" unterteilt, die sich von Sensor zu Sensor erstre- 
cken. Bei elf Sensorpositionen je Messachse ergeben sich also jeweils zehn Einzelelemente, die eine Art Stabzug bilden, vgl. Bild 3.8.

a)

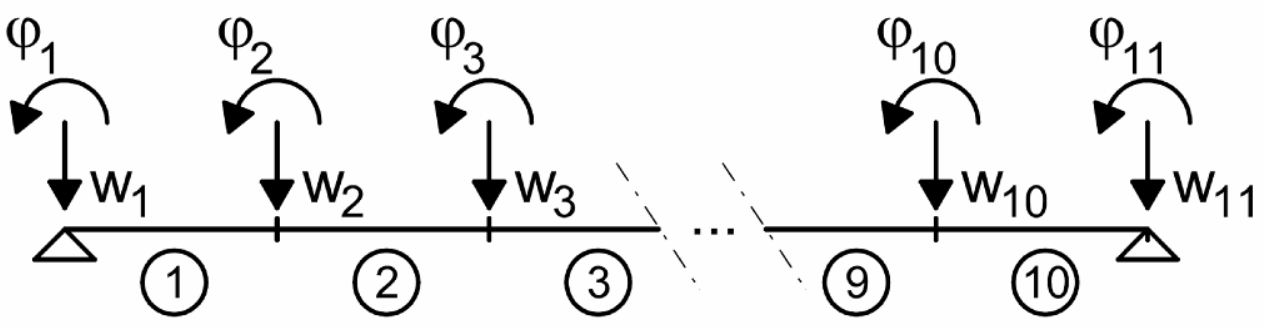

b)

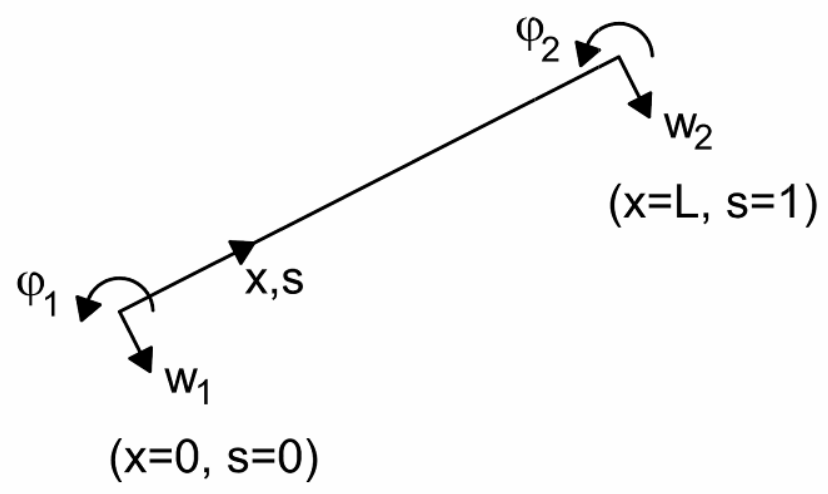

\section{Bild 3.8: a) Diskretisierung der vier Messachsen mit „Einzelelementen“ b) Notation Einzelelement}

Mit der Unterteilung in Einzelelemente wird in Anlehnung an die Finite Elemente Methode eine Diskretisierung des Systems vorgenommen, die es erlaubt, Beziehungen kinematischer Größen matriziell auszudrücken. Die Positionen der Sensoren sowie die Auflager markieren dabei die Elementknoten des diskreten Modells.

Ausgehend von einer polynomischen Ansatzfunktion für die Durchbiegung eines einzelnen Elements der Form

$$
w(s)=a_{1}+a_{2} \cdot s+a_{3} \cdot s^{2}+a_{4} \cdot s^{3}
$$

wobei $0 \leq \mathrm{s} \leq 1$ die lokale Koordinate des Einzelelements darstellt, lässt sich, wie in o. g. Publikationen detailliert beschrieben, folgender Zusammenhang zwischen den Dehnungen und den Durchbiegungen in den Elementknoten ableiten:

$$
\frac{1}{h_{0}} \cdot \underline{\varepsilon}=\underline{C} \cdot \underline{w}
$$


Die Dehnungen $\varepsilon_{\mathrm{i}}$ und Durchbiegungen $\mathrm{w}_{\mathrm{i}}$ werden darin vektoriell ausgedrückt. Dabei entspricht die Anzahl der Vektorkomponenten der Anzahl der wesentlichen Systemfreiheitsgrade. Bei den in Bild 3.8a dargestellten Verdrehungen handelt es sich um unwesentliche Freiheitsgrade, die mittels Kondensationstechniken [35] aus der Vektorgleichung eliminiert werden können. Unter der Annahme, dass Dehnungen und Durchbiegungen an den Auflagern vernachlässigbar klein sind, beträgt die Anzahl der wesentlichen Systemfreiheitsgrade im vorliegenden Fall neun ( $w_{2}$ bis $w_{10}$ ), siehe Bild 3.9. Matrix $C$ in Gleichung (3-4) ist folglich eine quadratische $(9,9)$-Matrix.

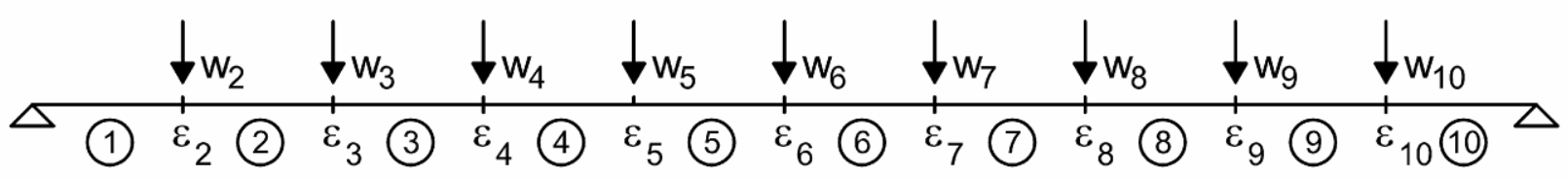

Bild 3.9: Monitoring Erfttalbrücke: Der Berechnung von Systemverformungen zugrunde liegendes diskretes System mit Kennzeichnung der wesentlichen Freiheitsgrade $w_{i}$ und der korrespondierenden Dehnungen $\varepsilon_{\mathrm{i}}$ aus Gleichung (3-4)

Der Parameter $h_{0}$ in Gleichung (3-4) bezeichnet den Abstand von der Systemschwerelinie der WIB-Platte zu dem Ort, an dem die Dehnungen gemessen werden, d. h. zur Unterseite der einbetonierten Stahlträger, siehe Bild 3.10.

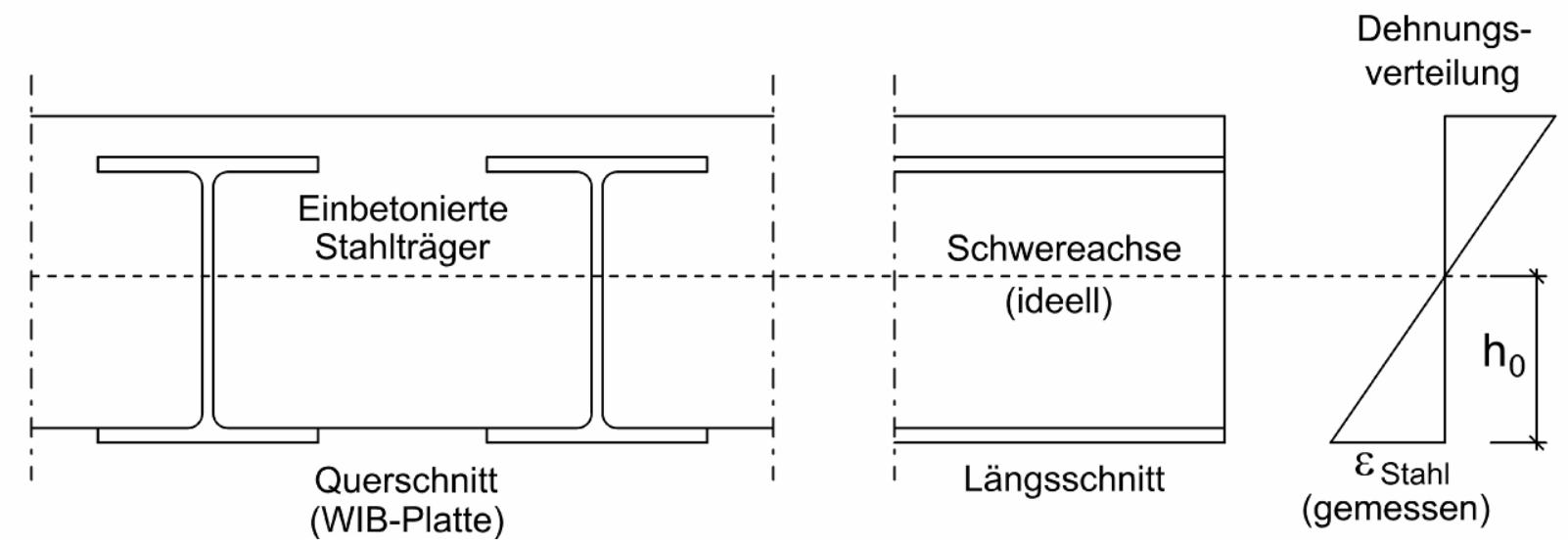

Bild 3.10: Quer- und Längsschnitt der WIB-Platte mit der geometrischen Größe $h_{0}$ als Abstand zwischen der Systemschwerelinie und der Unterseite der einbetonierten Stahlträger

Dieser Parameter wurde mit Hilfe eines modalanalytischen Ansatzes unter Verwendung sowohl von gemessenen Dehnungs- als auch von gemessenen Beschleunigungssignalen für jede Messachse (vgl. Bild 3.4a) separat ausgewertet. Dazu wurden Ausschwingkurven nach Überfahrtereignissen analysiert, die als 
Frequenzanteile fast ausschließlich die Grundeigenfrequenz enthalten. Es wurde näherungsweise angenommen, dass $h_{0}$ über die Messachse konstant ist. Da die Lage der Systemschwerelinie bzw. $h_{0}$ von der Geometrie des wirksamen Querschnitts abhängt und maßgeblich durch zusätzliche bauliche Elemente beeinflusst wird, die mehr oder weniger schubfest an den nackten WIBQuerschnitt angeschlossen sind, ergeben sich für jede der vier Messachsen unterschiedliche $\mathrm{h}_{0}$-Werte, vgl. Tabelle 3.3.

Tabelle 3.3: Monitoring Erfttalbrücke: Messtechnisch ermittelte Lagen der $h_{0}$-Werte in den vier Messachsen (Mittelwerte aus je fünf ausgewerteten Abklingkurven) [49]

\section{Achse 1 Achse 2 Achse 3 Achse 4}

\begin{tabular}{llllll}
\hline Mittelwert & 755 & 660 & 655 & 875 & $\mathrm{~mm}$ \\
\hline
\end{tabular}

Während sich für die innenliegenden Achsen 2 und 3 Werte ergeben, die mit rund $665 \mathrm{~mm}$ etwa $20 \%$ größer sind als der rechnerische Wert von $550 \mathrm{~mm}$, der sich bei ausschließlicher Berücksichtigung des in Bild 3.10 dargestellten reinen WIB-Querschnitts ergibt, wurden für die am Rand befindlichen Messachsen 1 und 4 deutlich höhere Werte ermittelt. Dieses Ergebnis ist vor dem Hintergrund verständlich, dass sich die Messachsen 1 und 4 unterhalb der Randkappen befinden, deren steifigkeitsbeeinflussende Wirkung signifikant ist. In Achse 1, unterhalb der weniger stark dimensionierten Randkappe (siehe Bild 3.3b), ergibt sich mit $780 \mathrm{~mm}$ ein $\mathrm{h}_{0}$-Wert, der etwa $40 \%$ größer ist als der o. a. rechnerische Wert von $550 \mathrm{~mm}$. In Achse 4, in der sich die stärker dimensionierte Randkappe mit Kabelkanal und Schallschutzwand befindet, wird dieser Wert sogar um $60 \%$ überschritten.

Um die Zuverlässigkeit des oben skizzierten Verfahrens zu überprüfen, wurden im Rahmen des Forschungsprojektes DETAILS [52] experimentelle Untersuchungen durchgeführt. Dazu wurde im Versuchslabor ein $12 \mathrm{~m}$ langer WIBTräger hergestellt und sowohl statisch als auch dynamisch beansprucht. Zur Messung von Beschleunigungen und Dehnungen wurde eine Messeinrichtung verwendet, die prinzipiell der Messkonfiguration beim Brückenmonitoring entspricht und somit die Voraussetzungen zur Anwendung des oben skizzierten Verfahrens erfüllt. Die Sensoren wurden mit einem Abstand untereinander von etwa 1,5 m entlang der Mittelachse angeordnet.

Neben Dehnungen und Beschleunigungen wurden mit Seilzugsensoren auch die Durchbiegungen des Versuchsträgers während der verschiedenen Belas- 
tungssituationen gemessen. Somit konnte ein Vergleich der nach oben dargestelltem Verfahren bestimmten Verformungsgrößen mit den direkt gemessenen Werten vorgenommen werden. Dabei zeigte sich eine ausgesprochen gute Übereinstimmung von berechneten und gemessenen Durchbiegungen. Näheres zu den Experimenten ist ebenfalls [49] zu entnehmen.

Im Rahmen der Messauswertung wird die Berechnung der Durchbiegungen in den vier Messachsen mit Hilfe einer FORTRAN Routine realisiert, die vom Auswertetool FOX aufgerufen wird. Eine animierte Darstellung der Verformungsverläufe während Zugüberfahrten ist z. B. mit dem Softwaretool WSTAB [62] möglich, siehe Momentaufnahme in Bild 3.11. Durch Implementierung des beschriebenen Verfahrens sind damit neben den Dehnungs- und Beschleunigungsverläufen somit auch die Durchbiegungsverläufe während Zugüberfahrten bekannt, die beispielsweise zur Verifizierung numerischer Simulationsrechungen verwendet werden können.

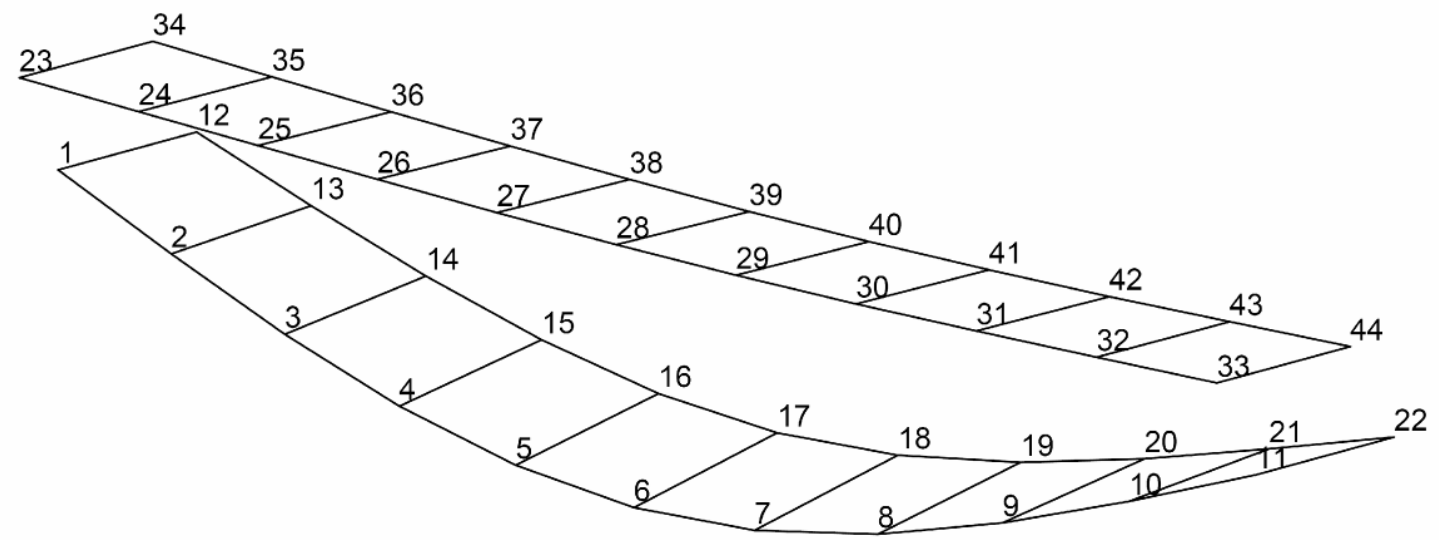

\section{Bild 3.11: Verformungsverlauf während einer Zugüberfahrt (räumliche Darstellung)}

\subsubsection{Maximalwerte von Beschleunigungen und Durchbiegungen}

In diesem und dem folgenden Abschnitt werden exemplarisch für den ICE-3 Zugtyp Monitoringergebnisse präsentiert. Zunächst werden Maximalwerte gemessener Beschleunigungen und Durchbiegungen behandelt, anschließend wird gezeigt, wie aus den gemessenen Zeitschrieben Eigenfrequenzen und Dämpfungswerte ermittelt wurden.

Um Zeitreihen von ICE-3 Zügen zu erhalten, wurden jeweils etwa 6000 Zugüberfahrten in den Zeiträumen vom 11. Januar 2010 bis zum 20. Februar 2010 (Winterdaten) sowie vom 21. April bis zum 22. Mai (Sommerdaten) ausgewertet. Die Winterdaten wurden bei zum Teil sehr niedrigen Temperaturen mit bis zu 
$-10^{\circ} \mathrm{C}$ aufgezeichnet. Dabei war das Schotterbett teilweise von einer dicken Schneeschicht bedeckt und infolge von Frost-Tauwechseln zeitweise gefroren.

Die Auswertung der Daten erfolgte automatisiert unter Verwendung des Auswertetools FOX. Im Nachgang wurde manuell sichergestellt, dass es sich bei den selektierten Zeitreihen tatsächlich um ICE-3 Überfahrten handelt.

Die Anzahl der identifizierten Überfahrtsereignisse ist in Tabelle 3.4 aufgeschlüsselt. Sommer- und Winterdaten bestehen jeweils aus etwa 100 Überfahrten je Überbau. Die nur sehr kleine Anzahl von Überfahrten im Winterzeitraum, die zur Ermittlung von Eigenfrequenzen und Dämpfung geeignet war, wird in Abschnitt 3.2.7 erklärt.

Tabelle 3.4: Monitoring Erfttalbrücke: Übersicht ausgewertete ICE-3 Überfahrten, Unterscheidung Sommer- I Winterdaten

\begin{tabular}{lcccc} 
& \multicolumn{2}{c}{ - Sommer - } & \multicolumn{2}{c}{ - Winter - } \\
\cline { 2 - 5 } & Überbau 1 & Überbau 2 & Überbau 1 & Überbau 2 \\
\hline $\begin{array}{l}\text { Gesamtanzahl der ausgewerteten } \\
\text { ICE-3 Überfahrten }\end{array}$ & 86 & 88 & 105 & 106 \\
\hline $\begin{array}{l}\text { davon geeignet zur Bestimmung von } \\
\text { Eigenfrequenz und Dämpfung }\end{array}$ & 51 & 62 & 7 & 8 \\
\hline
\end{tabular}

In Bild 3.12 sind die maximalen Beschleunigungen aufgetragen, die während der ausgewerteten ICE-3 Überfahrten aufgezeichnet wurden. Sie traten jeweils in Feldmitte auf - in der Regel am außen liegenden Rand des beanspruchten Überbaus (Achsen 1 und 4 in Bild 3.3d). Erwartungsgemäß nehmen die maximal auftretenden Beschleunigungen mit steigender Geschwindigkeit zu. Allerdings sind alle ermittelten Werte, den Bemessungsvorgaben im DINFachbericht [11] entsprechend, kleiner als der zulässige Grenzwert von $3,5 \mathrm{~m} / \mathrm{s}^{2}$.

Die üblichen Überfahrtgeschwindigkeiten während des Winterzeitraums sind mit etwa $200 \mathrm{~km} / \mathrm{h}$ wesentlich geringer als während der Sommermonate. Hier wurde die Erfttalbrücke sehr häufig mit Geschwindigkeiten um $250 \mathrm{~km} / \mathrm{h}$ befahren.

Während die Verteilung der Sommerdaten in sich konsistent ist und - wie später in Abschnitt 7 gezeigt wird - sehr gut mit rechnerischen Werten in Einklang gebracht werden kann, weisen die Winterdaten auffällige Unregelmäßigkeiten auf.

Insbesondere an Überbau 2 wurden für Geschwindigkeiten größer 200 km/h Beschleunigungen gemessen, die deutlich größer sind als die im Sommer aufgezeichneten Werte. Dies ist vermutlich auf die besonderen Witterungsverhält- 
nisse während des Winterzeitraums zurückzuführen, die unter anderem eine Änderung von Eigenfrequenz und kritischen Geschwindigkeiten bewirken können. Folgende Einflüsse sind in diesem Zusammenhang relevant:

- Zusätzliche Masse infolge Schnee und Eis

- Beeinflussung der Systemsteifigkeit durch Schnee und Eis

- Verändertes Dämpfungsverhalten infolge zusammengefrorener Schotterelemente

Die an Überbau 1 gemessenen Beschleunigungen sind tendenziell etwas größer im Vergleich zu Überbau 2. Dies ist vor dem Hintergrund plausibel, dass die Querschnittssteifigkeit von Überbau 1 mit $\mathrm{EI}=31280 \mathrm{MNm}^{2}$ (siehe hierzu Tabelle 7.1) aufgrund einer höheren Betonfestigkeit etwas größer ist als die Steifigkeit von Überbau 2, die nur 29220 MNm²$^{2}$ beträgt. Hinzu kommt, dass die Ausbauelemente von Überbau 1 aufgrund der erforderlichen Schallschutzwand massiver ausgeführt sind als bei Überbau 2, vgl. Bild 3.3d.

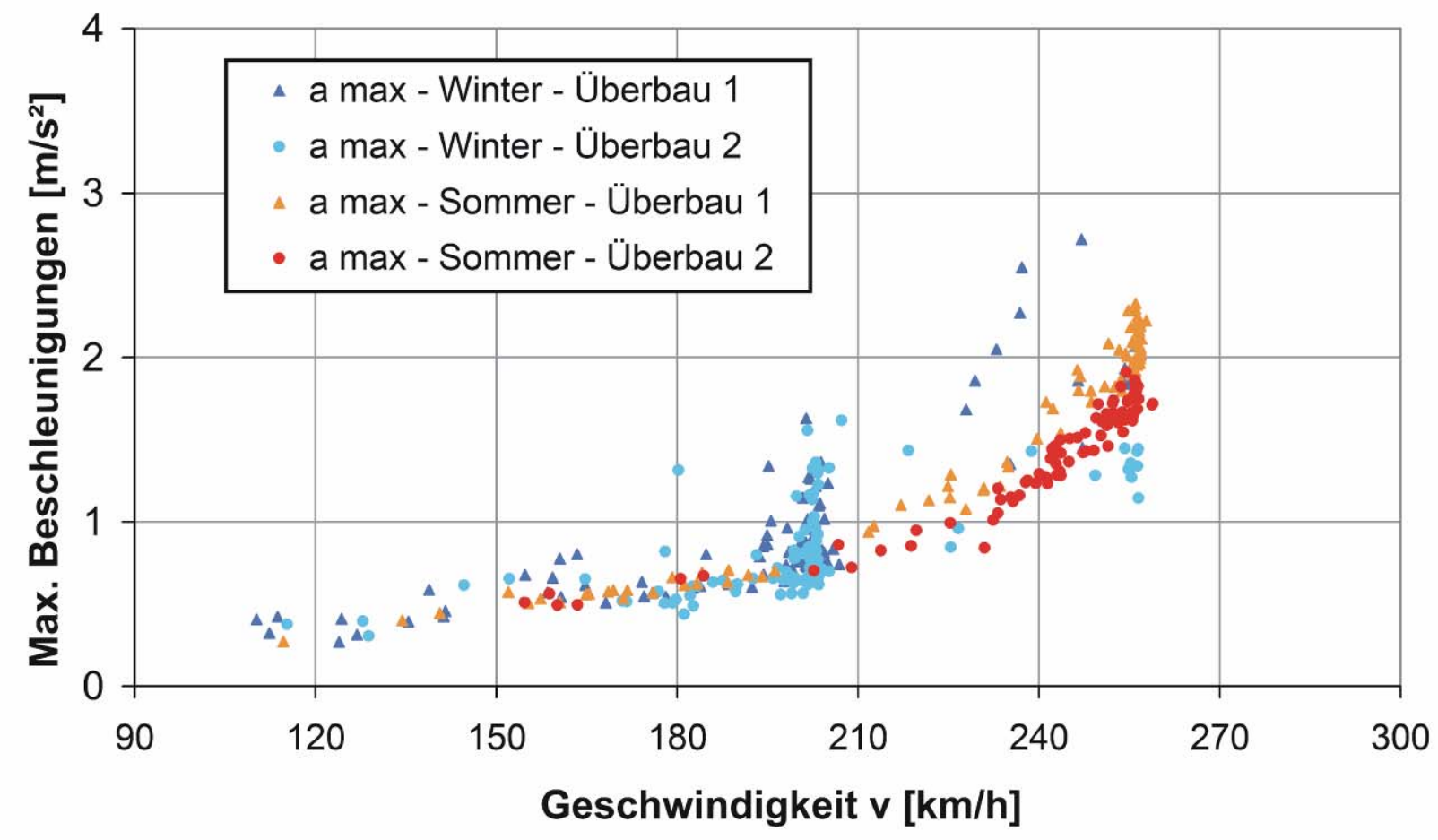

Bild 3.12: Monitoring Erfttalbrücke: Während ICE-3-Überfahrten gemessene maximale Beschleunigungen in Feldmitte des befahrenen Überbaus, Gegenüberstellung Sommer-/Winterdaten

Dieser Einfluss macht sich auch bei den in Bild 3.13 dargestellten Maximalwerten der Durchbiegungen bemerkbar, die nach dem in Abschnitt 3.2.5 beschriebenen Verfahren aus den Dehnungszeitschrieben abgeleitet wurden. Die maxi- 
male Durchbiegung von Überbau 1 in Feldmitte ist durchschnittlich etwa 1,0 mm größer als die entsprechende Durchbiegung von Überbau 2.

Wiederum zeigt sich eine wesentlich konsistentere Verteilung der Sommerdaten. Die Bandbreite der Durchbiegungen, die sich für einen bestimmten Geschwindigkeitsbereich ergibt, ist relativ klein im Vergleich zu den Winterdaten. Hier macht sich vermutlich erneut der Witterungseinfluss bemerkbar.

Die in diesem Abschnitt vorgestellten Monitoringergebnisse werden in Abschnitt 7.4 dazu verwendet, an der Erfttalbrücke durchgeführte numerische Überfahrtsimulationen zu verifizieren.

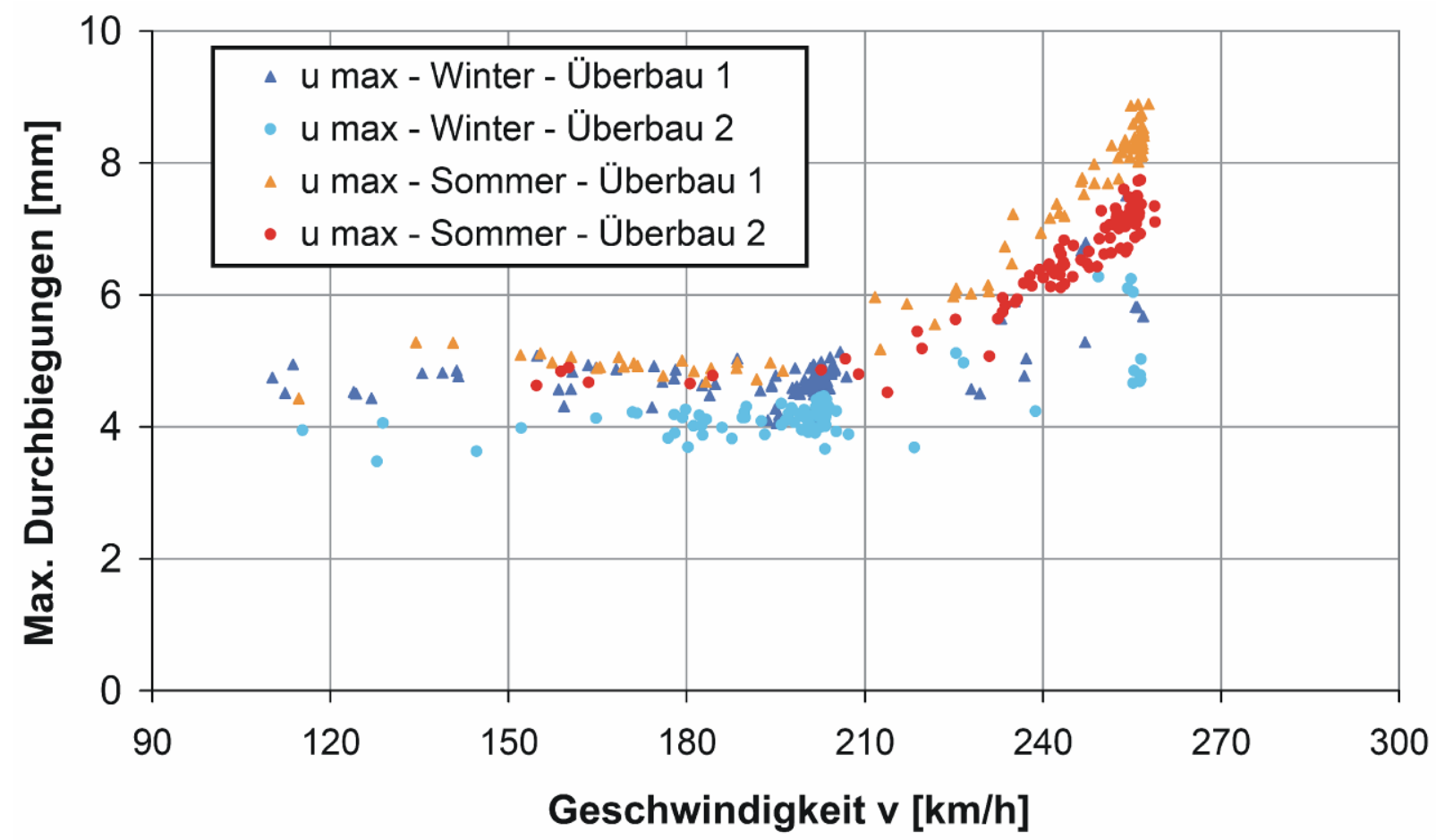

Bild 3.13: Monitoring Erfttalbrücke: Während ICE-3-Überfahrten gemessene maximale Durchbiegungen in Feldmitte des befahrenen Überbaus, Gegenüberstellung Sommer-/Winterdaten

\subsubsection{Eigenfrequenzen und Dämpfung}

Verlässt bei einer Zugüberfahrt das letzte Drehgestell die Brücke, so erfolgt anschließend ein freies Ausschwingen des Systems - in der Regel in der Grundeigenfrequenz. Die dabei gemessenen Abklingkurven können dazu verwendet werden, Grundeigenfrequenz und Systemdämpfung zu bestimmen. Unter Annahme einer geschwindigkeitsproportional gedämpften Schwingung ergibt sich eine periodische Funktion der Zeit, deren Amplitude mit einem Anfangswert $x_{0}$ beginnt und exponentiell mit der Zeit abnimmt, vgl. Bild 3.14. 


$$
x(t)=x_{0} \cdot \cos \omega_{D} t \cdot e^{-\delta t}
$$

$\delta$ ist darin die Abklingkonstante, $\omega_{D}$ die Kreisfrequenz des gedämpften Systems gemäß

$$
\omega_{D}=\sqrt{1-D^{2}} \cdot \omega_{0}
$$

mit der ungedämpften Kreiseigenfrequenz $\omega_{0}$.

Die Abklingkonstante $\delta$ in Gleichung (3-5) kann als Produkt des Lehr'schen Dämpfungsmaßes D (Prozentsatz der kritischen Dämpfung), das im Bauwesen üblicherweise zur Beschreibung der Dämpfung verwendet wird, und $\omega_{0}$ ausgedrückt werden:

$$
\delta=D \cdot \omega_{0}
$$

Zugüberfahrt Thalys - Dehnungen

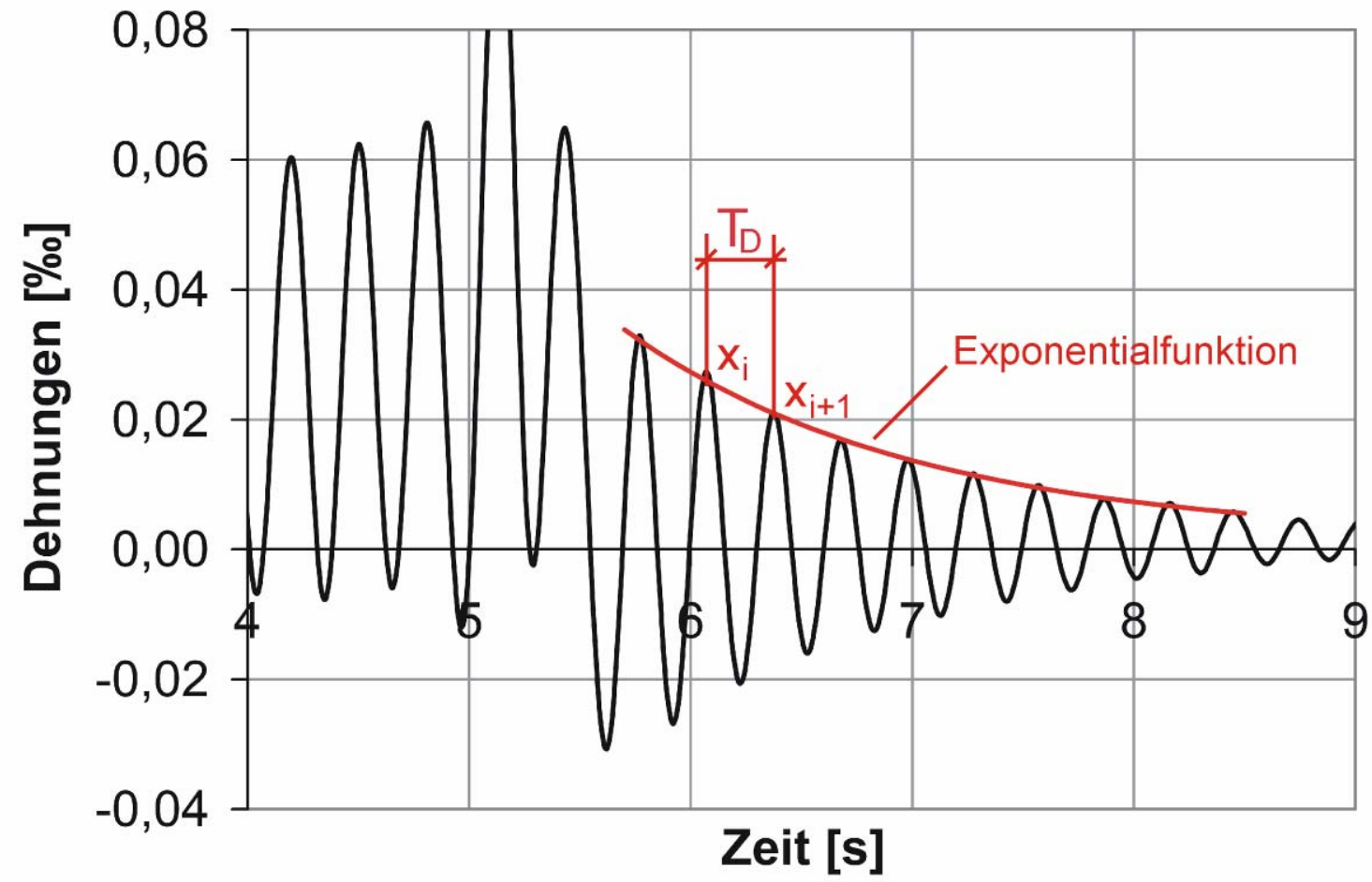

Bild 3.14: Zur Bestimmung von Eigenfrequenz und Dämpfung

Wird das Verhältnis zweier aufeinander folgender Amplitudenmaxima gebildet, so ergibt sich

$$
\frac{x_{i}}{x_{i+1}}=\frac{e^{-\delta i_{i}}}{e^{-\delta \delta_{i+1}}}=e^{\delta T_{D}}
$$


mit der Schwingungsperiode $T_{D}$ des gedämpften Systems als zeitlichem Abstand der aufeinander folgenden Amplituden:

$$
T_{D}=t_{i+1}-t_{i}=\frac{2 \pi}{\omega_{D}}
$$

Der Exponent $\delta \cdot T_{D}$ wird logarithmisches Dekrement genannt:

$$
\Lambda=\delta \cdot T_{D}=\ln \frac{x_{i}}{x_{i+1}}
$$

Unter Verwendung der Gleichungen (3-6), (3-7) und (3-9) ergibt sich

$$
\Lambda=\delta \cdot T_{D}=D \cdot \omega_{0} \cdot \frac{2 \pi}{\omega_{D}}=\frac{D}{\sqrt{1-D^{2}}} \cdot 2 \pi
$$

wobei für gering gedämpfte Systeme, wie hier der Fall, gilt:

$$
D \cong \frac{\Lambda}{2 \pi}
$$

Werden nicht zwei aufeinander folgende Amplitudenmaxima betrachtet, sondern die Maxima $x_{i}$ und $x_{i+n}$ zum Zeitpunkt $t_{i}$ und $t_{i+n}$ (also nach $n$ Schwingungszyklen), so ergibt sich für das logarithmischen Dekrement:

$$
\Lambda=\frac{1}{n} \cdot \ln \frac{x_{i}}{x_{i+n}}
$$

Vor der Analyse der Abklingkurven im Rahmen der Messauswertung mit dem Programm FOX wird das entsprechende Signal tiefpassgefiltert, um Störungen durch Frequenzanteile zu unterdrücken, die signifikant größer als die relevante Grundeigenfrequenz sind.

Zur Bestimmung der Dämpfungswerte $D$ werden bei der Auswertung der gemessenen Abklingkurven zwei unterschiedliche Ansätze verfolgt, wobei jeweils eine bestimmte Anzahl von Amplitudenmaxima $\mathrm{n}_{\text {Amp }}$ berücksichtigt wird:

- Gemäß Gleichung (3-10) werden die $\left(\mathrm{n}_{\mathrm{Amp}}-1\right)$ logarithmischen Dekremente der jeweils aufeinander folgenden Amplitudenmaxima bestimmt. Aus diesen Werten wird der Mittelwert gebildet und anschließend das Lehr'sche Dämpfungsmaß D nach Gleichung (3-12) ermittelt.

- Die Abklingkonstante $\delta$ in Gleichung (3-5) wird direkt bestimmt, indem eine $z u$ den $n_{\text {Amp }}$ Amplitudenmaxima passende Exponentialfunktion nach dem curve-fitting Prinzip bestimmt wird. Die Lehr'sche Dämpfung ergibt sich dann aus Gleichung (3-7). 
Beide Methoden liefern annähernd gleiche Ergebnisse. Die in der vorliegenden Arbeit dargestellten Ergebnisse wurden unter Anwendung der zweiten Methode erzeugt.

Die Grundeigenfrequenz des Systems kann unmittelbar aus dem zeitlichen Abstand der aufeinander folgenden Amplitudenmaxima berechnet werden - der Schwingungsperiode $T_{D}$ des gedämpften Systems, vgl. Gleichung (3-9). Für gering gedämpfte Systeme gilt dann:

$$
\omega_{0} \cong \omega_{D}=\frac{2 \pi}{T_{D}}
$$

Dieser Wert wird ebenfalls unter Berücksichtigung der $\mathrm{n}_{\text {Amp }}$ Amplitudenmaxima ermittelt, indem für $T_{D}$ der Mittelwert der $\left(n_{A m p}-1\right)$ gemessenen Schwingungsperioden eingesetzt wird.

Damit eine Zugüberfahrt zur Auswertung von Grundeigenfrequenz und Dämpfung verwendet wird, muss sie sich durch Erfüllung der folgenden beiden Kriterien, die im Rahmen der automatisierten Auswertung überprüft werden, qualifizieren. Das freie Ausschwingen nach der Überfahrt muss gekennzeichnet sein durch

- eine ausreichend hohe Amplitude zu Beginn des Ausschwingvorgangs

- eine ausreichende Anzahl an nachfolgenden Schwingungsamplituden, die eine bestimmte Größenordnung nicht unterschreiten

Grenzwerte für beide Kriterien werden in der Konfigurationsdatei des Auswertetools FOX spezifiziert.

Die bereits im vorangegangenen Abschnitt in Bezug auf maximale Systemantworten ausgewerteten ICE-3 Überfahrten wurden auch dazu verwendet, gemäß oben skizziertem Verfahren Eigenfrequenz- und Dämpfungswerte zu bestimmen. Die Ergebnisse sind in Bild 3.15 dargestellt, wobei erneut Winter- und Sommerdaten getrennt aufgetragen wurden.

Betrachtet man zunächst die Sommerdaten, ergibt sich erneut ein recht einheitliches Bild ohne auffällige Unregelmäßigkeiten. Die etwas geringere Steifigkeit von Überbau 1 spiegelt sich in den tendenziell etwas niedrigeren Grundeigenfrequenzen im Vergleich zu Überbau 2 wider, siehe Bild 3.15a. Außerdem ist die Systemdämpfung von Überbau 1 mit rund $2,5 \%$ etwa einen halben Prozentpunkt geringer als die Dämpfung von Überbau 2, siehe Bild 3.15b. Grund hierfür könnten neben der massiver ausgeführten Randkappe von Überbau 2 zusätzli- 
che bauliche Elemente wie Schallschutzwand und Kabelkanäle sein, vgl. Bild 3.3d.

a)

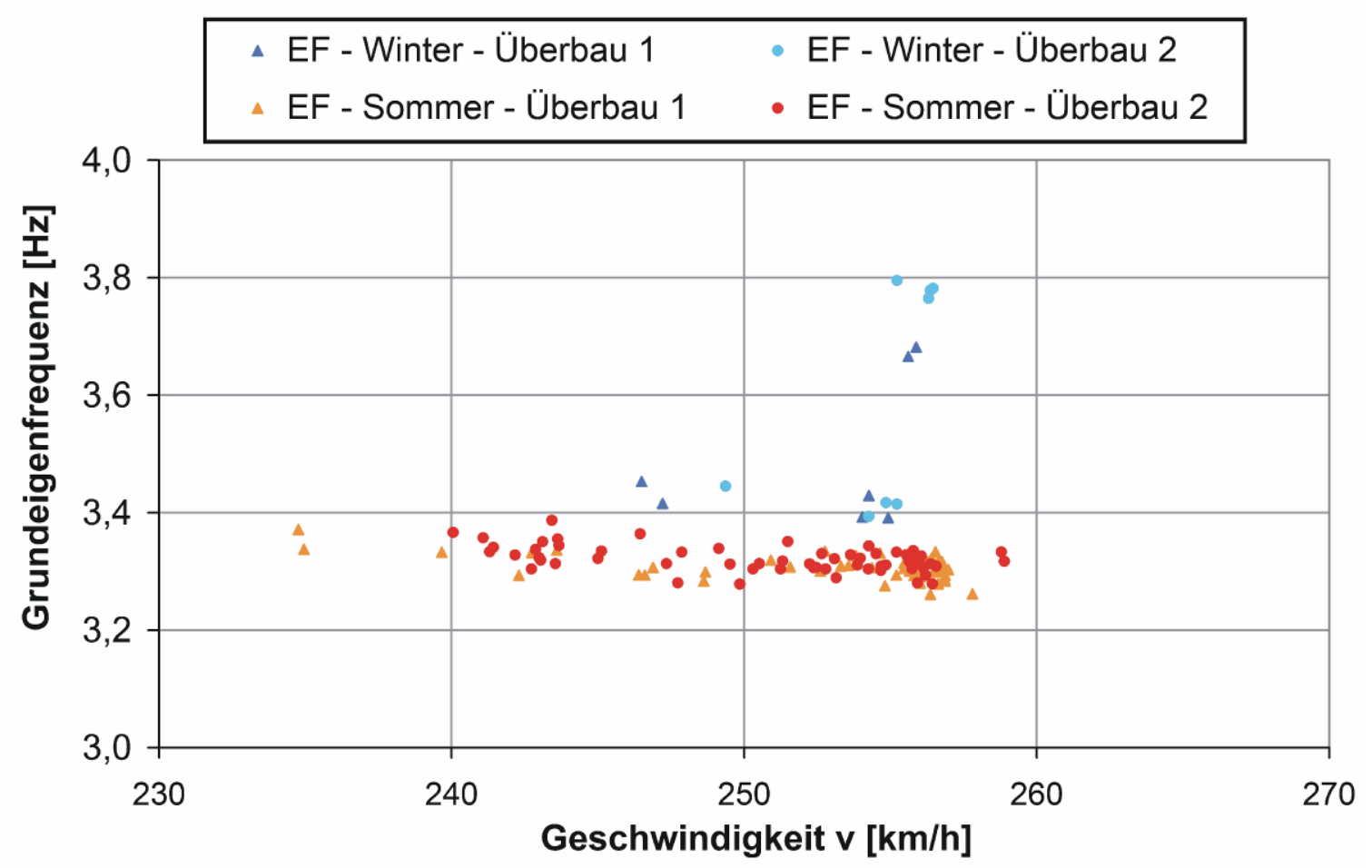

b)

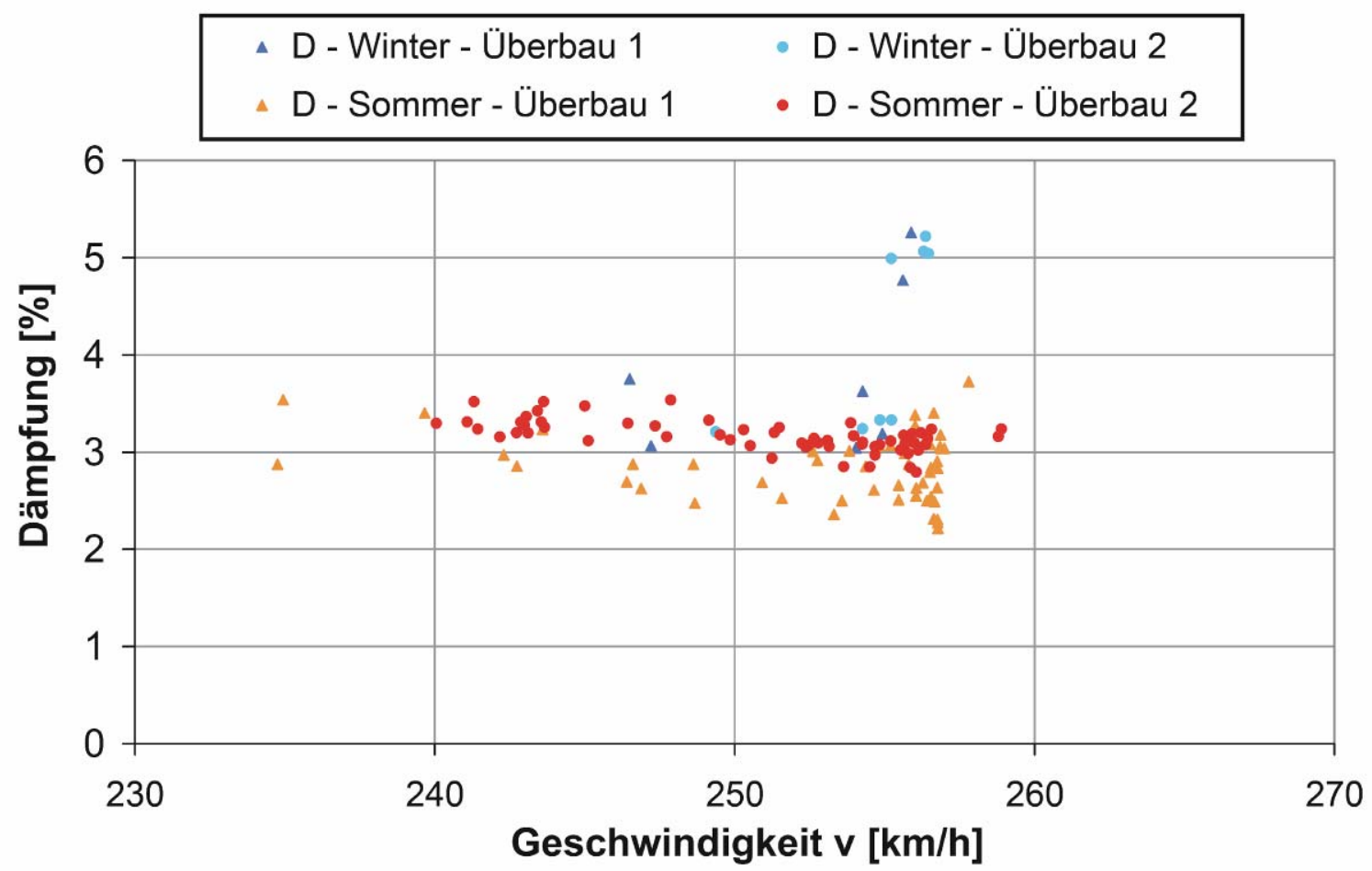

Bild 3.15: Monitoring Erfttalbrücke: Während des Ausschwingvorgangs nach ICE3-Überfahrten gemessene Werte von a) Grundeigenfrequenzen b) Lehr'sche Dämpfung; Gegenüberstellung jeweils der Messwerte aus Sommer und Winter 
In Bezug auf die während des Sommerzeitraums auftretenden Eigenfrequenzen ist bemerkenswert, dass mit durchschnittlich rund $3,3 \mathrm{~Hz}$ ein signifikant niedrigerer Wert ermittelt wurde als im Rahmen der in [50] behandelten Messkampagne, die ebenfalls unter sommerlichen Bedingungen durchgeführt wurde. Dort ergab sich eine Grundeigenfrequenz von etwa $3,7 \mathrm{~Hz}$. Allerdings wurden im Rahmen jener Messkampagne ambiente Schwingungen analysiert, $d . h$. Schwingungen mit sehr kleinen Amplituden, die nicht aus Zugüberfahrten, sondern aus ambienten Anregungsquellen wie Wind oder unter der Brücke stattfindendem Straßenverkehr resultierten.

Diese Diskrepanz zwischen den unterschiedlichen Messergebnissen deutet auf ein nichtlineares amplitudenabhängiges Schwingungsverhalten hin. Offenbar ist die Steifigkeit des nahezu in Ruhe befindlichen Systems signifikant größer als das durch Zugverkehr angeregte System. Dieser Sachverhalt könnte auf den nicht-linearen Steifigkeitseinfluss der Schotterschicht zurückzuführen sein. Größere Schwingungsamplituden des Brückenüberbaus könnten zu einer Instabilität der Schotterschicht führen, was eine geringere Steifigkeit zur Folge hätte. In Abschnitt 4, der die experimentellen Untersuchungen an Eisenbahnschotter behandelt, wird diese Überlegung aufgegriffen. Auch dort wurde ein amplitudenabhängiges Verhalten des Schotters beobachtet. Die Autoren von [51] [53] haben ähnliches festgestellt.

Ein Vergleich der Sommer- mit den Winterdaten ist schwierig, weil nur relativ wenige der während des Winterzeitraums aufgezeichneten Zeitreihen dazu geeignet waren, Eigenfrequenz- und Dämpfungswerte aus Abklingkurven nach Überfahrtsereignissen zu bestimmen, vgl. hierzu auch Tabelle 3.4. Die Mehrzahl der Zeitreihen enthielten nur sehr schwach ausgeprägte bzw. überhaupt keine Abklingkurven. Dies ist auf den Aufhebungseffekt zurückzuführen, der in Abschnitt 2.2 behandelt wurde. Nach Gleichung (2-5) ergibt sich im Falle der Erfttalbrücke unter Zugrundelegung einer Eigenfrequenz von $3,5 \mathrm{~Hz}$ die folgende erste Aufhebungsgeschwindigkeit:

$$
v_{\text {cancel }}(j=1)=\frac{2}{3} \cdot n_{0} \cdot L_{S}=\frac{2}{3} \cdot 3,5 \mathrm{~Hz} \cdot 24,6 \mathrm{~m} \cdot 3,6=207 \mathrm{~km} / \mathrm{h}
$$

Diese Geschwindigkeit von rund $200 \mathrm{~km} / \mathrm{h}$ liegt genau in dem Geschwindigkeitsbereich, mit dem die Erfttalbrücke im ausgewerteten Winterzeitraum hauptsächlich befahren wurde. Der Aufhebungseffekt führt dazu, dass die Brücke nach Beendigung der Überfahrt praktisch in Ruhe bleibt, ohne nennenswert auszuschwingen. Dieses spezielle Verhalten wurde in Bild 2.6 dargestellt. Fast alle während des Winterzeitraums aufgezeichneten Zeitreihen wiesen ein 
Ausschwingverhalten auf, das mit dem in Bild 2.6a dargestellten Verlauf vergleichbar ist. Eine Auswertung nach vorgenannter Methode war damit nicht möglich.

Die wenigen Überfahrten während des Winterzeitraums, die im Hinblick auf Grundeigenfrequenz und Systemdämpfung ausgewertet wurden, zeigen, wie schon im Hinblick auf Beschleunigungen und Durchbiegungen beobachtet (siehe Abschnitt 3.2.6), ein uneinheitliches Bild. Die Grundeigenfrequenzen der beiden Überbauten variieren zwischen 3,4 und $3,8 \mathrm{~Hz}$, während die Dämpfungswerte zwischen 3,0 und 5,5\% liegen. Dieses relativ breite Ergebnisspektrum wird erneut auf winterliche Witterungseinflüsse zurückgeführt. Insbesondere das stark variierende Dämpfungsverhalten lässt vermuten, dass Schnee und Eis einen nennenswerten Einfluss auf die Brückendynamik haben und die Systemdämpfung deutlich vergrößern können. Um diesen Sachverhalt weiter abzuklären, sind weiterführende Untersuchungen erforderlich.

\subsection{Schlussfolgerungen}

Zusammenfassend können die folgenden wesentlichen Schlussfolgerungen aus den Messergebnissen abgeleitet werden:

- Die bisherigen Erfahrungen, dass bei kurz bis mittelweit spannenden Eisenbahnbrücken die tatsächlichen dynamischen Systemparameter Eigenfrequenz und Dämpfung signifikant von rechnerischen bzw. normativen Werten abweichen, werden durch die vorliegenden Messberichte bestätigt.

- Weiterhin wird bestätigt, dass die Abweichung zwischen den theoretischen und den tatsächlichen Steifigkeiten mit abnehmender Spannweite größer wird. Bei kleinen Stützweiten bis etwa 12,5 m übersteigt die effektive Steifigkeit die Steifigkeit des „nackten“ Überbauquerschnitts um das 3- bis 4-fache. Bei größeren Stützweiten bis $25 \mathrm{~m}$ verringert sich der Abstand auf etwa das 1,5- bis 2,5-fache.

- Im Rahmen der Ableitung von Brückendurchbiegungen aus Dehnungsmessungen an der Erfttalbrücke konnte eindeutig belegt werden, dass bauliche Zusatzelemente die Querschnittseigenschaften beeinflussen. Es wurde festgestellt, dass die Lage der Nulllinie im Querschnitt bzw. der Parameter $h_{0}$ umso stärker vom rechnerischen Wert am reinen WIBQuerschnitt abweicht, je größer die Steifigkeit zusätzlicher Ausbauelemente ist, die sich oberhalb des untersuchten Querschnittsabschnitts befinden. 
- Bei eingleisigen und zweigleisigen Überbauten konnten keine nennenswerten Unterschiede bezüglich zusätzlicher Steifigkeitsbeiträge festgestellt werden.

- Mit Bezug auf die Dämpfung sind keine eindeutigen Abhängigkeiten von baulichen Randbedingungen festzustellen. Unabhängig von der Stützweite ist bei allen untersuchten Überbauten die gemessene Dämpfung mindestens $35 \%$ größer als der nach Norm anzusetzende Wert.

- Schallschutzwände haben keinen unmittelbar nachweisbaren Effekt auf die Systemdämpfung.

- Die Untersuchungsergebnisse, die im Hinblick auf Witterungseinflüsse gewonnen wurden, sind uneinheitlich. Während die Messungen der Deutschen Bahn keinen signifikanten Einfluss belegen, bestätigen die Ergebnisse der Monitoringkampagne an der Erfttalbrücke die bereits zitierten Erfahrungen anderer Autoren, dass sich bei winterlichen Witterungsverhältnissen die dynamische Systemeigenschaften gegenüber sommerlichen Bedingungen verändern können.

Es ist allgemein unstrittig, dass konstruktive Gegebenheiten ursächlich für die Fehleinschätzung tatsächlich vorhandener Biegesteifigkeiten sind. Ungeklärt ist bisher allerdings, in welchem Maße welche bauliche Randbedingung am Steifigkeitszuwachs beteiligt ist. Dies lässt sich aus den Messergebnissen nicht unmittelbar ableiten.

Um diesbezüglich genauere Kenntnisse zu erlangen, wurden ergänzende numerische Untersuchungen durchgeführt. Sie sollten auch Aufschluss darüber geben, wie sich die Randbedingungen am effektivsten im numerischen Modell umsetzen lassen. Die Ergebnisse der numerischen Studien werden in Abschnitt 5 vorgestellt. Zunächst jedoch werden im folgenden 4. Abschnitt die Ergebnisse experimenteller Untersuchungen an Eisenbahnschotter präsentiert. Die aus den Versuchen abgeleiteten Materialparameter wurden dazu genutzt, die Kopplung benachbarter eingleisiger Überbauten infolge durchgehender Schotterschichten in numerischen Modellen abzubilden. 


\section{Experimentelle Untersuchungen an Eisenbahnschotter}

\subsection{Einführung}

In Hochgeschwindigkeitsstrecken der Deutschen Bahn wird bevorzugt die Feste Fahrbahn eingebaut [41]. Zwar sind die Investitionskosten wesentlich höher, doch ergeben sich in HGV-Strecken geringere Unterhaltskosten für das Gleis der Festen Fahrbahn als für den Schotteroberbau. In dieser Hinsicht stellen die WIB-Brücken des HGV-Schienennetzes eine Besonderheit dar, weil sie fast ausschließlich mit Schotteroberbau ausgerüstet sind. Bei den meisten dieser Brücken handelt es sich um nebeneinander (teilweise auch versetzt) angeordnete eingleisige Brückenüberbauten, die durch eine Bauwerksfuge getrennt sind. Diese Bauweise hat den Vorteil, dass die Brückendecks einzeln überholt oder erneuert werden können, ohne den Betrieb auf der benachbarten Strecke zu beeinträchtigen, vgl. Bild 4.1.

a)

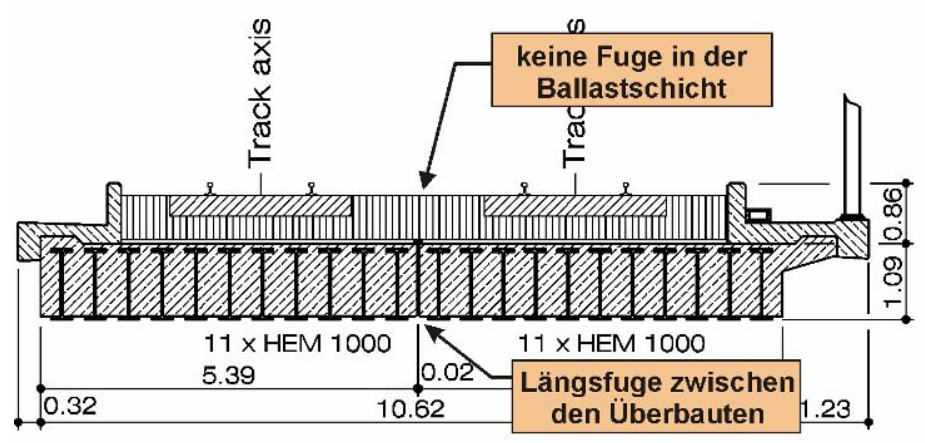

b)

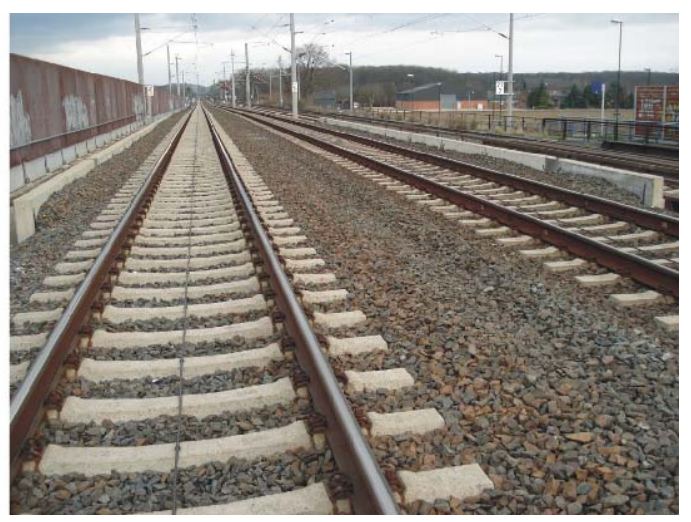

Bild 4.1: Typischer WIB-Überbau mit durchgehender Schotterschicht a) Querschnitt b) örtliche Gegebenheiten

Allerdings kann im Hinblick auf das dynamische Verhalten des Brückenbauwerks nicht von einer baulichen Trennung der Überbauten ausgegangen werden, da das auf den Brückendecks befindliche Schotterbett durchgehend ausgeführt wird, vgl. Bild 4.1. Daraus ergibt sich eine bauliche Kopplung der Überbauten, die nicht zu vernachlässigende Interaktionseffekte während Zugüberfahrten zur Folge hat, wie Bild 4.2 zeigt. Die dargestellten Messaufzeichnungen stammen aus der Monitoringkampagne an der WIB-Brücke EÜ Erfttalstraße (siehe Abschnitt 3.2) und belegen einen signifikanten Lasteintrag in den unbelasteten Überbau, woraus sich eine dynamische Gesamtanregung beider Brückenhälften ergibt. Es besteht großes Interesse, diesen mechanischen Effekt im Rahmen numerischer Simulationsrechnungen zu berücksichtigen, um das reale 
Tragwerksverhalten realistisch abbilden zu können. Wie im Folgenden gezeigt wird, lässt sich die Kopplung über den Schotter näherungsweise mit Hilfe linearelastischer Federn beschreiben, die eine Art Schubsteifigkeit des Schotterbetts repräsentieren.

a)

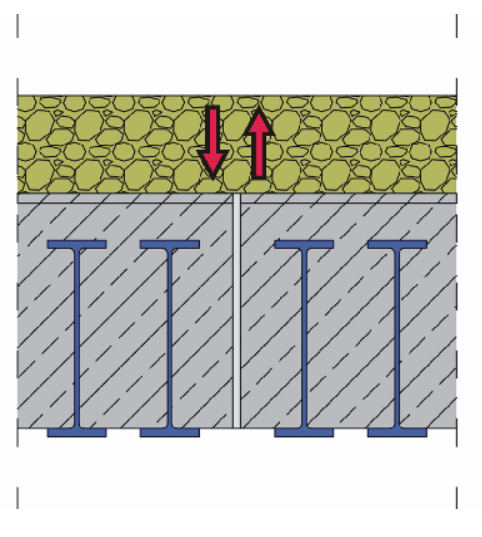

b)

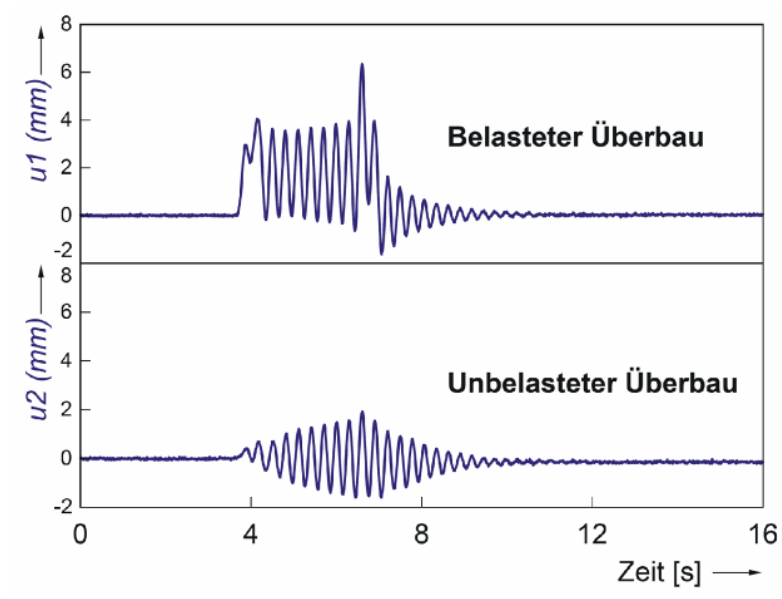

c)

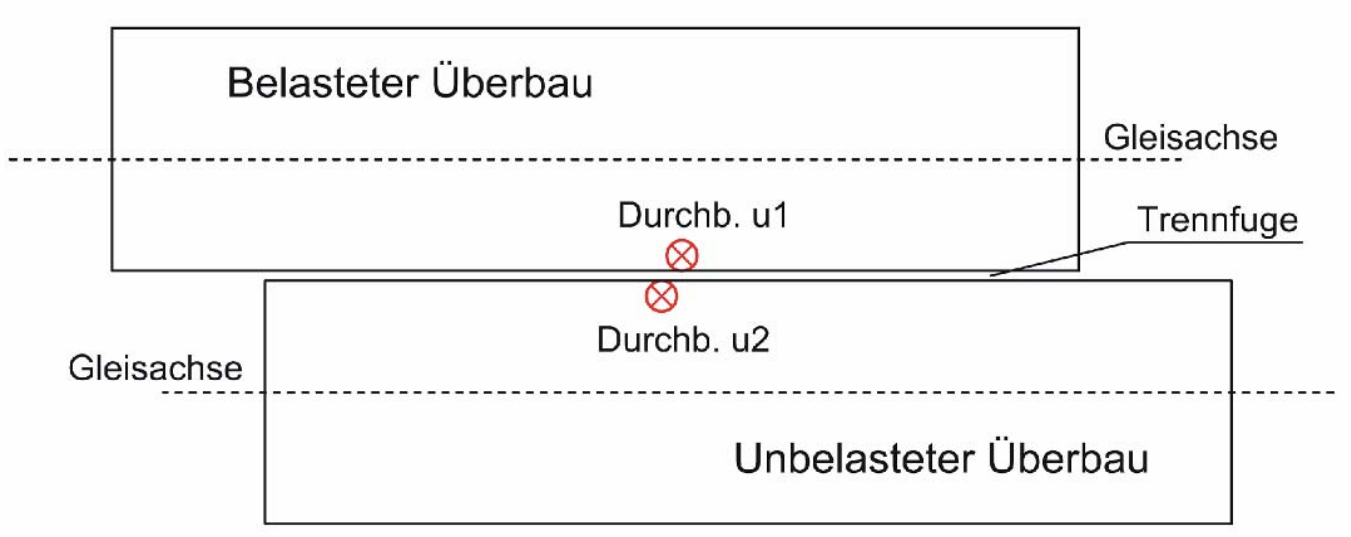

Bild 4.2: a) Kopplung von WIB-Überbauten durch Schotter b) Durchbiegungen eines belasteten bzw. eines unbelasteten Überbaus c) Draufsicht gekoppelte Überbauten

Allerdings gibt es derzeit keine experimentellen Ergebnisse zum Kopplungsverhalten eingleisiger Brückenüberbauten infolge durchgehender Schotterschichten. Es wurden daher im Rahmen des Europäischen Forschungsprojektes DETAILS [52] Versuche durchgeführt, um das Last-Verformungsverhalten von gekoppelten Schotterschichten zu untersuchen, siehe auch [45].

Das Hauptziel der experimentellen Untersuchungen war die Identifizierung eines mechanischen Parameters, der zur Beschreibung der Koppelwirkung in numerischen Berechnungen herangezogen werden kann. Daneben sollte der Einfluss des Schotters auf die globale Biegesteifigkeit einer mit Schotterbett versehenen Struktur untersucht werden. Außerdem konnten im Rahmen der 
Versuchsauswertung Informationen zum Dämpfungsverhalten des Schotters gewonnen werden.

In den folgenden Abschnitten werden die durchgeführten Versuche beschrieben und die Versuchsergebnisse dargestellt. Anschließend wird erläutert, wie die Ergebnisse bei der Erstellung numerischer Brückenmodelle verwendet werden können. Es wird weiterhin der Zusammenhang zu bestehenden Berechnungsansätzen hergestellt, die es bereits erlauben, horizontale Beanspruchungen aus Zugverkehr (Lasten aus Bremsen und Anfahren) durch das Schotterbett abzuleiten. In Abschnitt 4.5 wird dann gezeigt, wie die Versuchsergebnisse im Rahmen numerischer Berechnungen verwendet werden können.

\subsection{Versuchsaufbau}

Der Versuchskörper bestand aus einem zweiteiligen Stahlkasten, dessen Trennfuge entlang der Mittelachse verlief, siehe Bild 4.3. Beide Kastenhälften wurden auf jeweils zwei Stahlträger (IPE 200) gesetzt und mit ihnen verschweißt. Die so gebildeten Biegeträger wurden gelenkig gelagert und hatten eine Spannweite von 4,1 m bei einer Breite von 1,2 m (je Hälfte 0,6 m). Die rechnerische Biegesteifigkeit des Gesamtquerschnitts betrug $\mathrm{EI}=13490 \mathrm{kNm}^{2}$, woraus sich eine Grundeigenfrequenz der Einfeldsysteme von etwa $32 \mathrm{~Hz}$ ergab (ohne Schotter). Die Konstruktion wurde so ausgeführt, dass die Seitenwände keinen nennenswerten Beitrag zur Biegesteifigkeit leisteten.
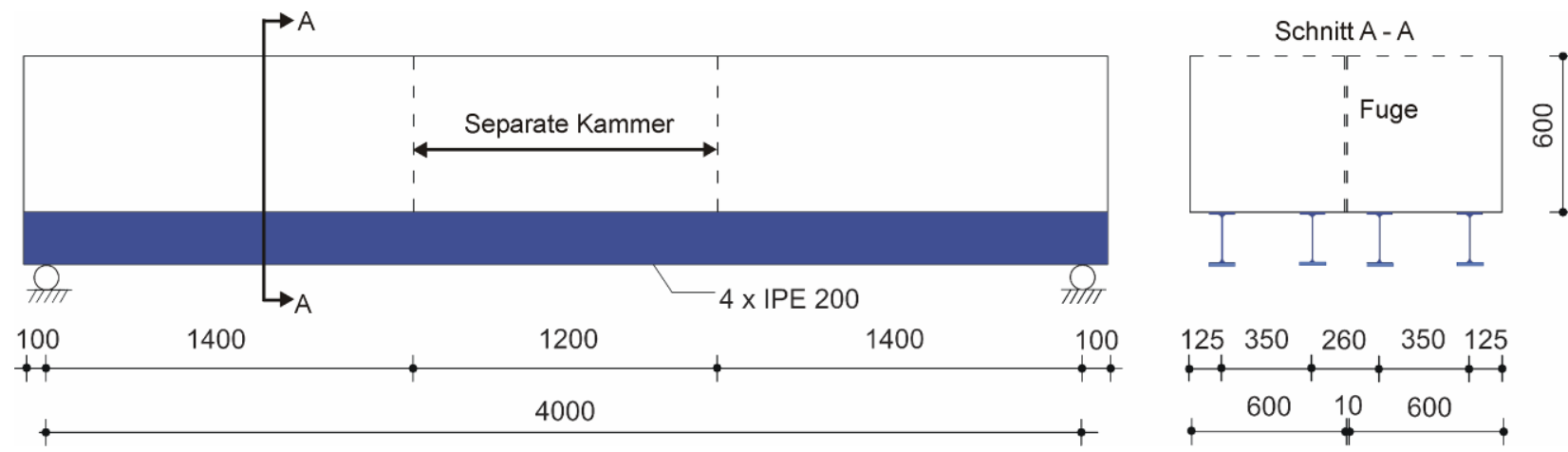

Bild 4.3: Längs- und Querschnitt der Versuchsapparatur

Der Stahlkasten wurde mit Eisenbahnschotter gefüllt, der standardmäßig auf Hochgeschwindigkeitsstrecken der Deutschen Bahn verwendet wird und sich u.a. auch auf der Eisenbahnbrücke EÜ Erfttalstraße befindet. Dabei handelt es sich um einen Basalt Schotter Körnungstyp 1 (31,5 - 63,0 mm). Die Füllhöhe des Schotters betrug $50 \mathrm{~cm}$ und entsprach damit der üblichen maximalen Höhe von Schotterschichten im Bereich der Trennfuge nebeneinander liegender Ü- 
berbauten. Zur Verdichtung der Schotterschicht wurde eine gewöhnliche $11 \mathrm{kN}$ Rüttelplatte verwendet, siehe Bild 4.4d.

Auf den Bahnstrecken der Deutschen Bahn werden die oben liegenden Schotterkörner miteinander verklebt, um ausreichende Stabilität des Schotters während Zugverkehr zu gewährleisten und ein Verrutschen oder Aufwirbeln einzelner Körner zu verhindern. Eine solche Verklebung des Schotters wurde im Rahmen der Versuche nicht vorgenommen, da eine nennenswerte Beeinflussung der Versuchsergebnisse dadurch nicht zu erwarten war.

Bei Belastung einer Hälfte des mit Schotter gefüllten Versuchskörpers wurde die Situation auf einer Brücke des untersuchten Typs (durch Schotter gekoppelte, baulich getrennte Überbauten) simuliert. Über die Koppelfuge in der Schotterschicht, welche die einzige Verbindung der beiden Kastenhälften darstellte, fand ein Lasteintrag in die unbelastete Hälfte statt. Um ein Auseinanderdriften der beiden Hälften im Versuch zu verhindern, wurde der Versuchskörper auf mittlerer Höhe in Querrichtung abgestützt.

Durch das Einsetzen von Querschotten in den Stahlkasten konnte im mittleren Bereich des Kastens eine 1,2 $\mathrm{m}$ breite separate Kammer gebildet werden, die mit Schotter befüllt wurde, vgl. Bild 4.3 und Bild 4.4a. Damit sollte erreicht werden, dass die Relativverschiebungen in jedem Punkt der Kontaktfläche zwischen beiden Kastenhälften annähernd gleich groß waren. Diese Randbedingung war im Hinblick auf die vereinfachten zweidimensionalen mechanischen Modelle von Bedeutung, die bei der Versuchsauswertung zur Anwendung kamen - ein Aspekt, auf den in Abschnitt 4.3 näher eingegangen wird.

Im Rahmen der Versuche wurden sowohl quasi-statische als auch dynamische Lasten aufgebracht. Außerdem wurden Anregungen mit einem Impulshammer vorgenommen. Messtechnisch wurden die aufgebrachten Lasten sowie die resultierenden Verformungen des Versuchskörpers in Feldmitte erfasst. Indem die Durchbiegungen aller vier Träger gemessen wurden, konnten auch die Verdrehungen beider Kastenhälften bestimmt werden (Verwendung potentiometrischer Wegtaster vom Typ TR 25, Hersteller Novotechnik, Messungenauigkeit $0,05 \mathrm{~mm}$ ). Darüber hinaus wurden bei einigen Versuchsreihen Beschleunigungen beider Kastenhälften in Feldmitte gemessen (Verwendung kapazitiver Beschleunigungssensoren vom Typ M3701, Hersteller PCB, Messbereich $\pm 3 \mathrm{~g}$ ).

Die Messwerte wurden dazu verwendet, mit Hilfe vereinfachter mechanischer Modelle Steifigkeitskennwerte zur Lastübertragung zwischen den beiden Kastenhälften abzuleiten. Außerdem wurde der Steifigkeitsbeitrag des Schotterbetts zur Biegesteifigkeit des Versuchskörpers untersucht. Zu diesem Zweck wurde 
der gesamte Stahlkasten ohne Verwendung der Querschotte mit Schotter befüllt, so dass sich ein Einfeldträger mit konstantem "Querschnitt“ (Stahlquerschnitt mit Schotterbett) ergab. Die Impulsanregungen dienten dazu, Steifigkeits- und Dämpfungseigenschaften des vollständig mit Schotter gefüllten Kastens zu untersuchen.

a)

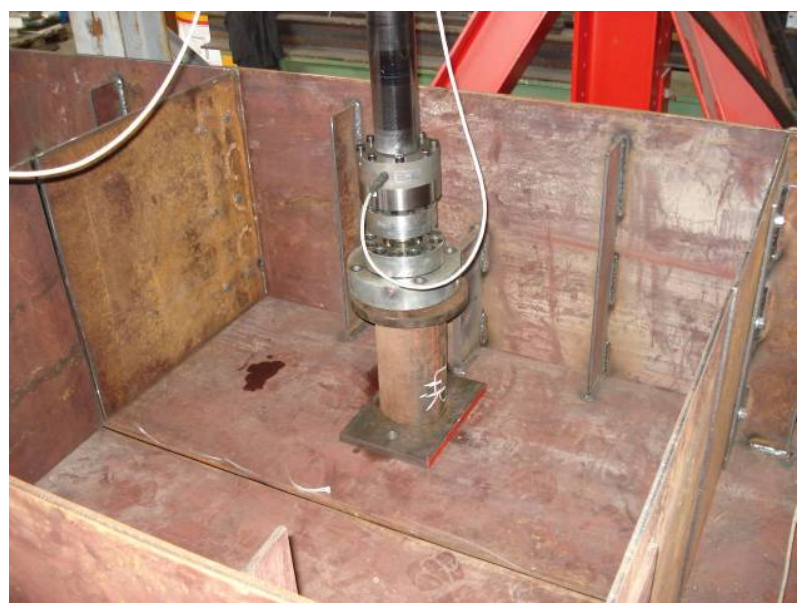

c)

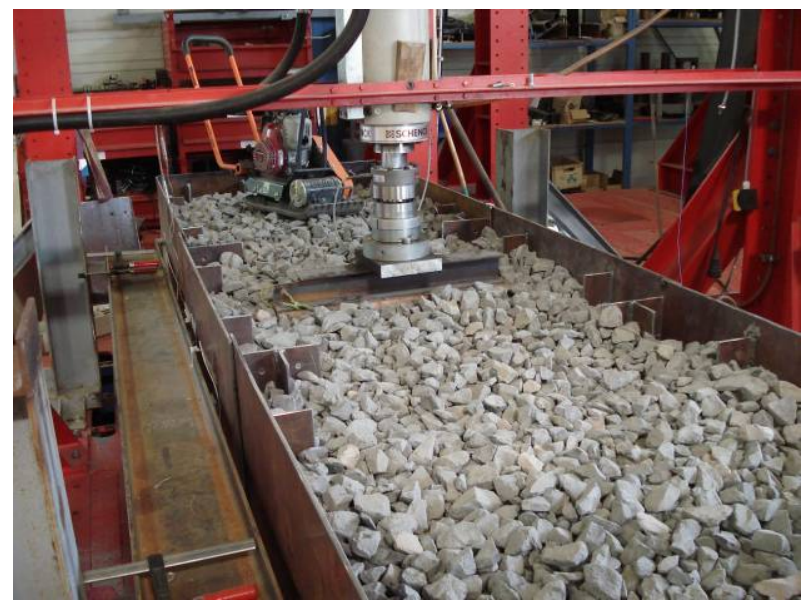

b)

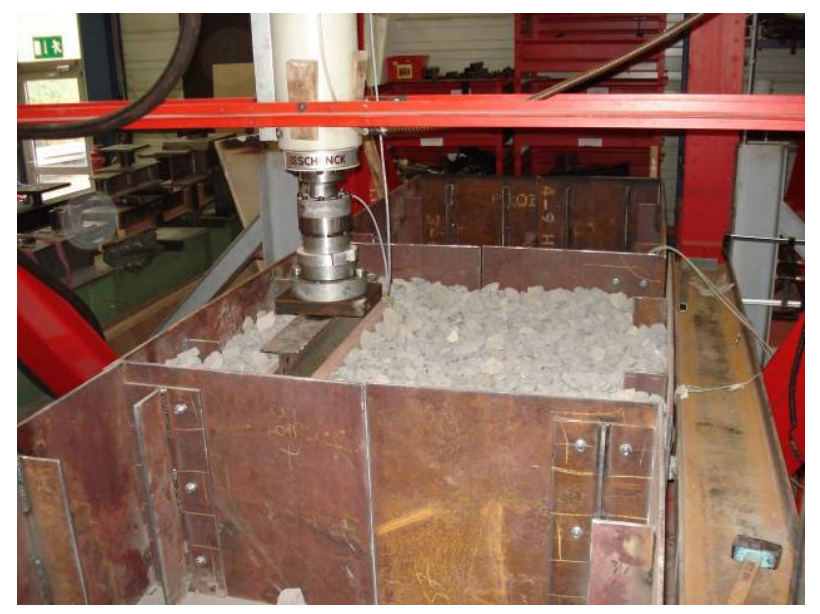

d)

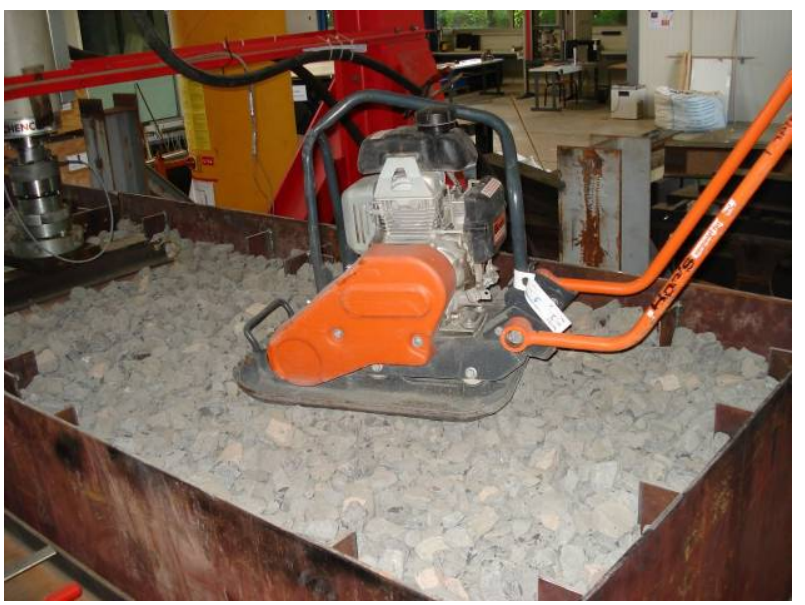

Bild 4.4: Durchführung der Versuche a) Belastung des leeren Stahlkastens (Testmodus M1) b) Belastung der mit Schotter gefüllten Mittelkammer (Testmodus M2 bzw. M3) c) vollständig gefüllter Kasten d) Rüttelplatte zur Verdichtung des Schotters

\subsection{Versuchsdurchführung und -auswertung}

Zur Bestimmung mechanischer Parameter wurden vier verschiedene Test-Modi (M1 - M4) durchgeführt, die in Tabelle 4.1 beschrieben sind. Mit jedem Modus wurde dabei jeweils ein Parameter bestimmt. 


\section{Tabelle 4.1: Beschreibung der vier Test-Modi M1-M4}

\begin{tabular}{|c|c|c|c|}
\hline Test modus & Beschreibung & Zielparameter & Versuchsreihen \\
\hline M1 & $\begin{array}{l}\text { - Kasten leer } \\
\text { - eine Kastenhälfte belastet }\end{array}$ & $\begin{array}{c}\mathrm{C}_{\mathrm{b} 1} \\
\text { (Biegesteifigkeit ohne Schotter) }\end{array}$ & $\begin{array}{c}4 \\
(\mathrm{~S} 1-\mathrm{S} 4)\end{array}$ \\
\hline M2 & $\begin{array}{l}\text { - Mittelkammer mit Schotter gefüllt } \\
\text { - eine Kastenhälfte belastet } \\
\text { - beide Kastenhälften frei beweglich }\end{array}$ & $\begin{array}{c}\mathrm{c}_{\mathrm{b} 2} \\
\text { (Biegesteifigkeit mit Schotter in } \\
\text { Mittelkammer) }\end{array}$ & $\begin{array}{c}4 \\
(\mathrm{~S} 1-\mathrm{S} 4)\end{array}$ \\
\hline M3 & $\begin{array}{l}\text { - Mittelkammer mit Schotter gefüllt } \\
\text { - eine Kastenhälfte belastet } \\
\text { - die andere Kastenhälfte nicht beweglich }\end{array}$ & $\begin{array}{c}\mathrm{C}_{\text {coup }} \\
\text { (Koppelsteifigkeit) }\end{array}$ & $\begin{array}{c}4 \\
(S 1-S 4)\end{array}$ \\
\hline M4 & $\begin{array}{l}\text { - Kasten vollständig mit Schotter gefüllt } \\
\text { - beide Kastenhälften gleichmäßig belastet } \\
\text { - beide Kastenhälften frei beweglich }\end{array}$ & $\begin{array}{l}\mathrm{C}_{\mathrm{b} 3} \\
\text { (Biegesteifigkeit mit vollständig } \\
\text { gefülltem Kasten) }\end{array}$ & $\begin{array}{c}2 \\
(\mathrm{~S} 3-\mathrm{S} 4)\end{array}$ \\
\hline
\end{tabular}

Die Steifigkeitsparameter $\mathrm{C}_{\mathrm{b} 1}-\mathrm{C}_{\mathrm{b} 3}$ (Biegung) und $\mathrm{c}_{\text {coup }}$ (Koppeleffekt) stehen für Federsteifigkeiten in vereinfachten zweidimensionalen mechanischen Modellen. Es handelt sich hierbei um Ersatzfedersteifigkeiten, die spezifische Steifigkeitseigenschaften des Versuchskörpers repräsentieren (siehe Klammerausdrücke hinter den einzelnen Zielparametern in Tabelle 4.1). Für die Testmodi M2 und M3 sind die entsprechenden Modelle in Bild 4.5 dargestellt. Dabei wird mit den Federsteifigkeiten $\mathrm{c}_{\mathrm{b} 2}$ (Schotter in Mittelkammer) bzw. $\mathrm{c}_{\mathrm{b} 3}$ (vollständig gefüllter Kasten) sowohl die Biegesteifigkeit der Stahlstruktur als auch der Zusatzbeitrag des Schotters abgebildet.

Der allgemeine Zusammenhang zwischen $c_{b}$ und der Biegesteifigkeit El eines Einfeldträgers mit konstantem Querschnitt kann wie folgt ausgedrückt werden. Eine Kraft $F$ in Feldmitte resultiert in einer Durchbiegung $z$ mit

$$
z=\frac{F \cdot L^{3}}{48 \cdot E I}
$$

wobei L die Spannweite des Einfeldsystems ist. Mit

$$
c_{b}=\frac{F}{Z}
$$

ergibt sich für $\mathrm{c}_{\mathrm{b}}$ :

$$
c_{b}=\frac{48 \cdot E I}{L^{3}}
$$


a)
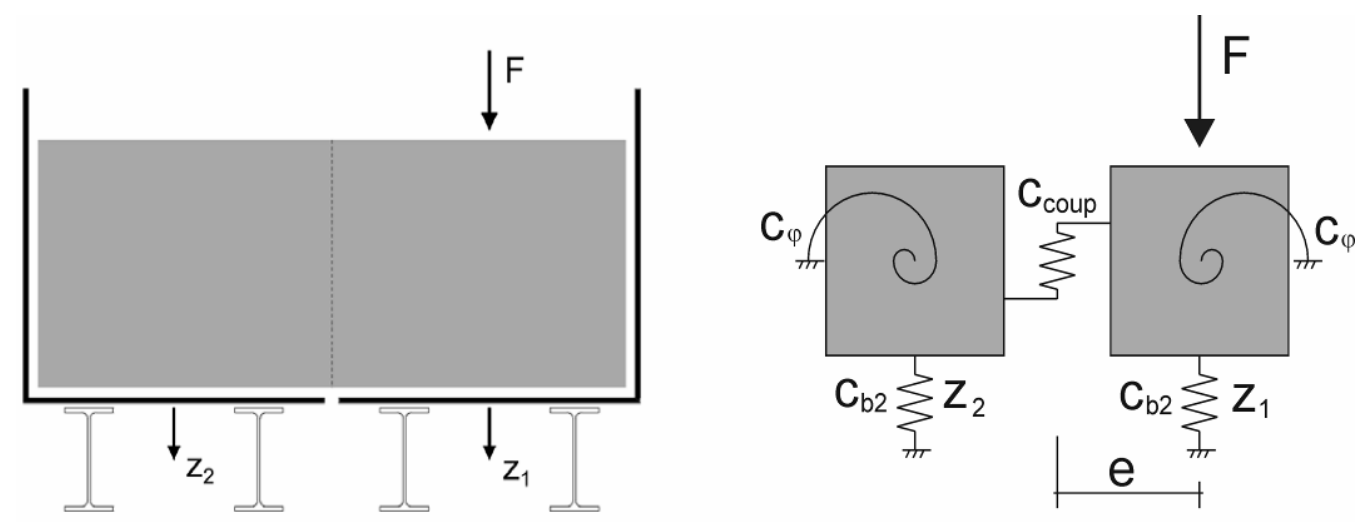

b)
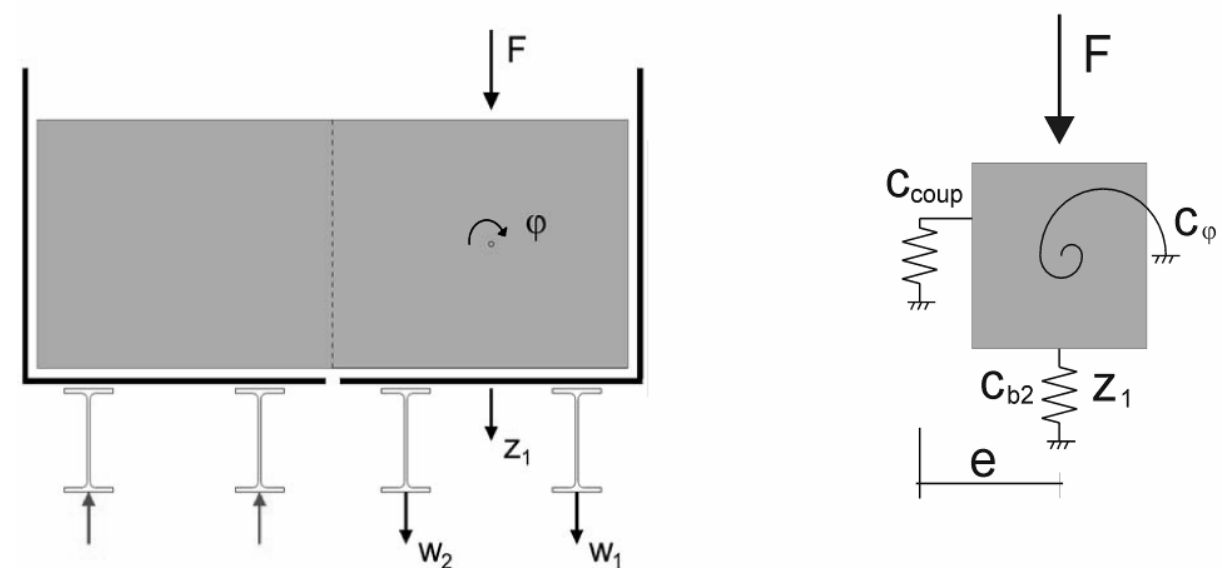

Bild 4.5: Test-Modi M2 und M3; Zugehörige mechanische Modelle

Nach Durchführung von Testmodus $\mathrm{M} 2$, bei dem sich beide Kastenhälften durchbiegen und verdrehen konnten (vgl. Bild 4.5) wurde der Parameter $\mathrm{C}_{\mathrm{b} 2}$ unter Annahme von Kräftegleichgewicht in vertikaler Richtung folgendermaßen berechnet:

$$
c_{b 2}=\frac{F}{z_{1}+z_{2}}
$$

Anschließend wurde Testmodus M3 durchgeführt, nachdem Durchbiegungen und Verdrehungen der unbelasteten Kastenhälfte durch entsprechende Unterstützungen verhindert wurden (vgl. Bild 4.5). Unter Verwendung von $\mathrm{c}_{\mathrm{b} 2}$ aus Testmodus M2 und der Verdrehung $\varphi$ der belasteten Kastenhälfte, berechnet aus $\mathrm{w}_{1}$ und $\mathrm{w}_{2}$ (siehe Bild 4.5b), wurde der Parameter $\mathrm{c}_{\text {coup }}$ ebenfalls unter Annahme von Kräftegleichgewicht in vertikaler Richtung wie folgt ermittelt:

$$
C_{\text {coup }}=\frac{F-c_{b 2} \cdot z_{1}}{z_{1}-e \cdot \varphi}
$$


wobei e den Abstand zwischen der Kontaktfläche beider Kastenhälften und der Mittelachse einer Kastenhälfte bezeichnet. Die Ersatzfedersteifigkeiten $c_{b 1}$ and $\mathrm{C}_{\mathrm{b} 3}$ wurden mit Hilfe der Testmodi M1 und M4 unter Verwendung der Gleichungen (4-2) und (4-4) bestimmt.

Durch Verwendung der zweidimensionalen mechanischen Modelle bei der Versuchsauswertung wird das tatsächlich dreidimensionale Systemverhalten vereinfacht. Insbesondere wird bei Herleitung von Gleichung (4-5) die Kraft in der Koppelfuge $F_{\text {coup }}$ unter Verwendung von Verformungsgrößen bestimmt, die in Feldmitte auftreten bzw. dort gemessen werden.

$$
F_{\text {coup }}=c_{\text {coup }} \cdot\left(z_{1}-e \cdot \varphi\right)
$$

Tatsächlich sind die Verformungen aber nicht konstant über die Trägerlänge. Während sie am Rand sehr klein werden, sind sie in Trägermitte maximal. Aus diesem Grund wurde bei Durchführung der Testmodi M2 und M3 die separate Mittelkammer eingesetzt. Die Kontaktfläche wird damit auf einen Bereich in Feldmitte beschränkt, so dass die Verformungen an jeder Stelle dieser Kontaktfläche annähernd gleich groß sind, vgl. Bild 4.6.

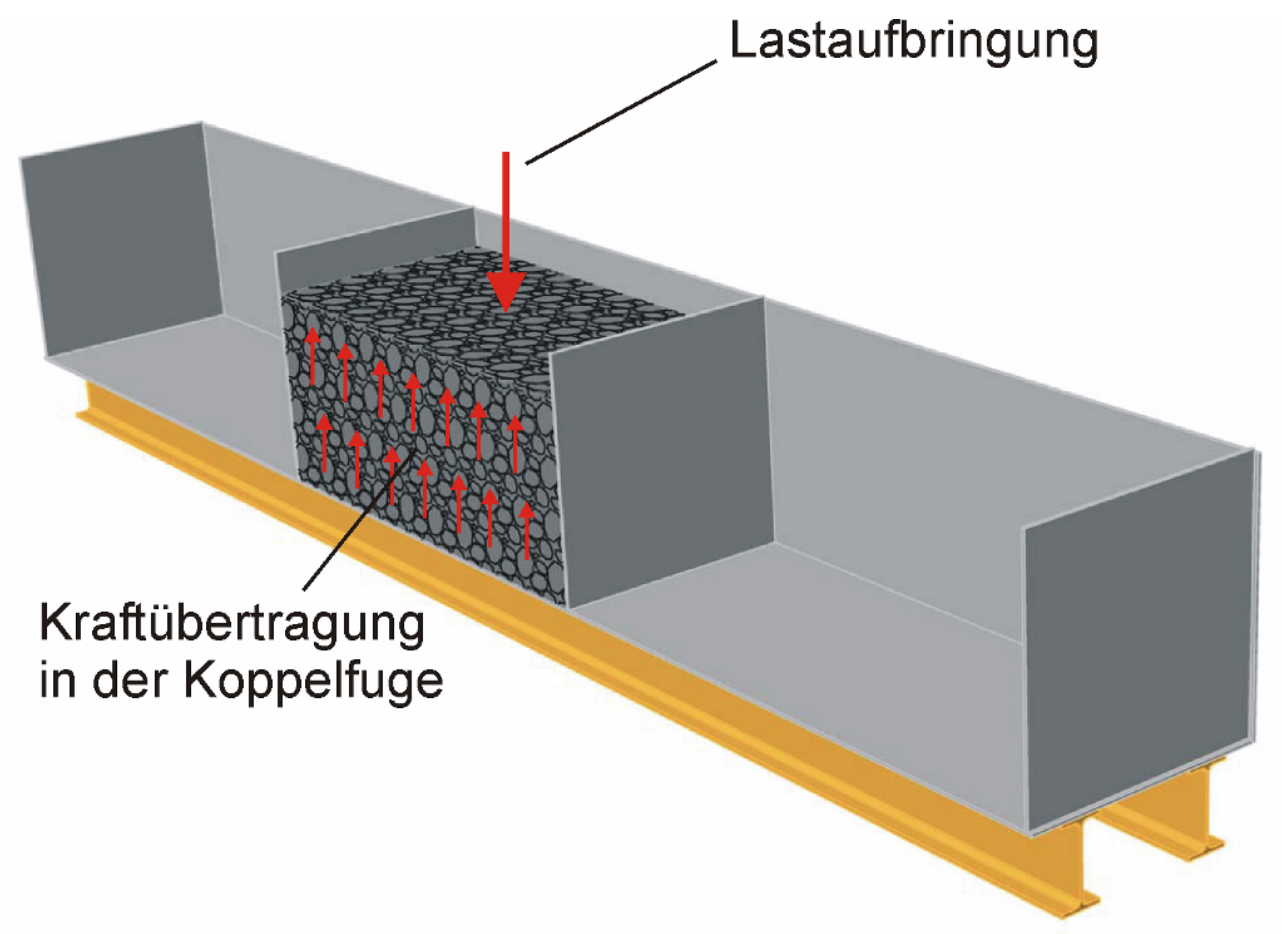

Bild 4.6: Kraftübertragung in der Koppelfuge bei Durchführung der Testmodi M2 und M3

Alle Testmodi wurden mehrmals durchgeführt, wobei sich insgesamt vier Versuchsreihen (Testserien S1-S4) ergaben, vgl. Tabelle 4.1. Die Testmodi M1 bis M3 (ohne Schotter oder Schotter in Mittelkammer) waren Gegenstand aller vier 
Versuchsreihen, während Testmodus M4 nur im Rahmen der letzten beiden Versuchsreihen durchgeführt wurde. Für jede Versuchsreihe wurde neuer ungebrauchter Schotter verwendet, da im Verlaufe der Versuche leichte Schädigungen der Schottersteine in Form von Abrieberscheinungen festgestellt wurden.

Die Versuche wurden lastgesteuert durchgeführt, wobei die Geschwindigkeit der Lastaufbringung bei den quasi-statischen Tests $2 \mathrm{kN} / \mathrm{s}$ betrug. Im Rahmen der dynamischen Versuche wurden sinusförmige Lastverläufe mit Amplituden von $\pm 5 \mathrm{kN}$ bis $\pm 20 \mathrm{kN}$ in einem Frequenzbereich von 0,5 bis $6,0 \mathrm{~Hz}$ aufgebracht. Mit diesen Belastungsfrequenzen wurden typische Grundeigenfrequenzen von mittelweit spannenden Eisenbahnbrücken erfasst, die bei bemessungsrelevanten Resonanzeffekten hauptsächlich angeregt werden.

a)

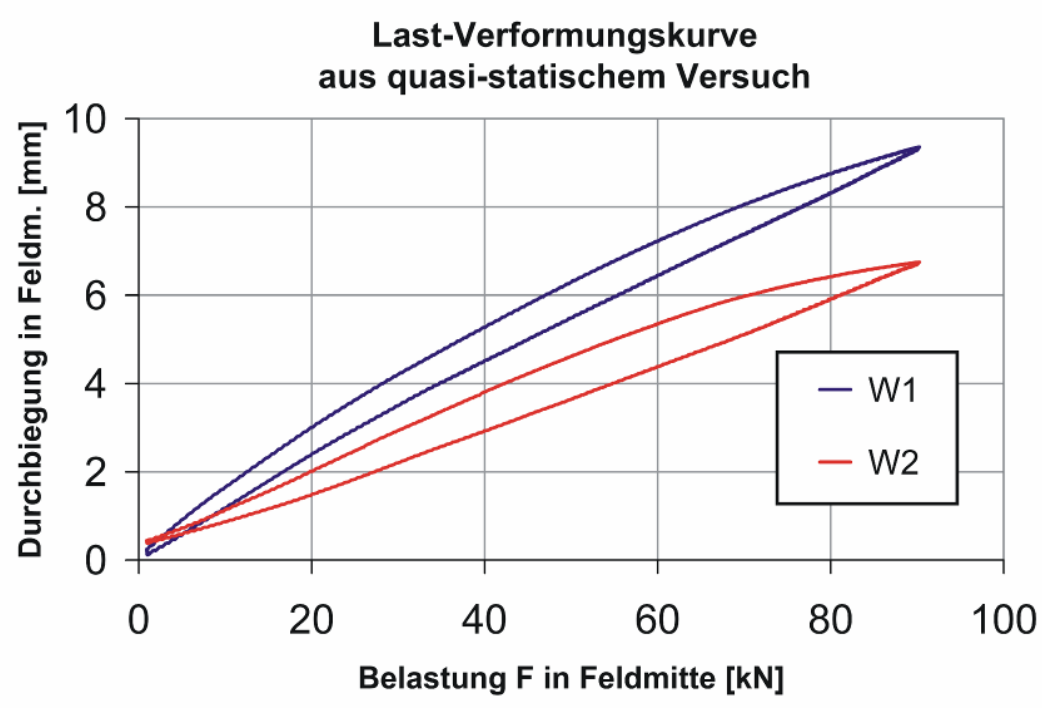

b)

Last-Verformungskurve aus zyklischem Versuch mit $3.0 \mathrm{~Hz}$

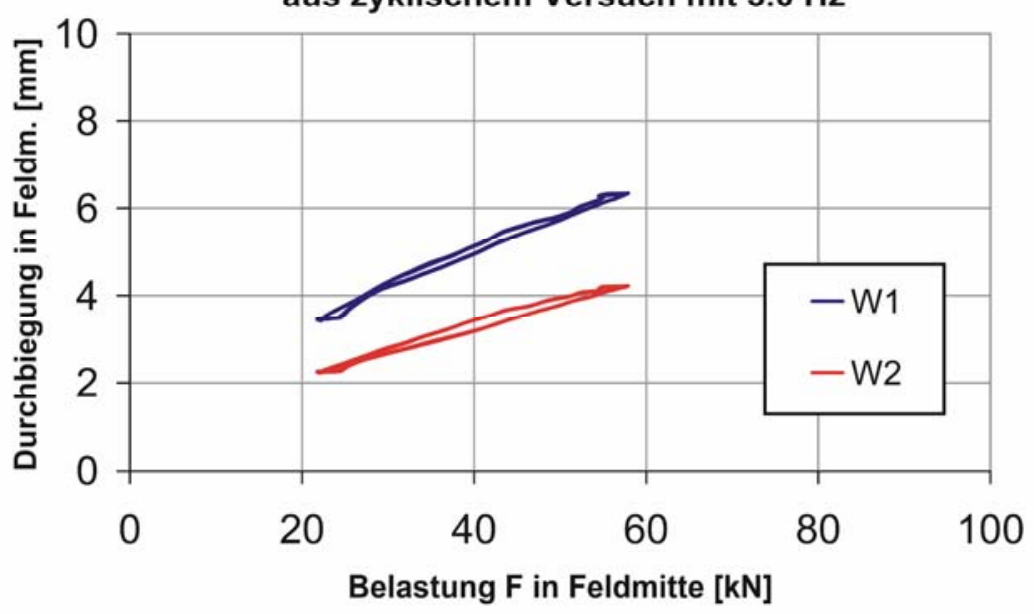

Bild 4.7: Exemplarischer Verlauf der Last-Verformungskurve aus a) quasi-statischen und b) dynamischen Versuchen (Testmodus M3, $w_{1}$ und $w_{2}$ sind in Bild $4.5 b$ definiert) 
In Bild 4.7 sind Last-Verformungskurven aus quasi-statischen und dynamischen Versuchen bei Durchführung von Testmodus M3 dargstellt (unbelastete Kastenhälfte in Ruhe), wobei sich die dynamische Kurve aus insgesamt 16 Lastzyklen zusammensetzt. Für den gesamten Lastbereich von maximal $100 \mathrm{kN}$, der während der Versuche aufgebracht wurde, konnte ein elastisches Verhalten mit visko-elastischen Einflüssen beobachtet werden, vgl. auch [14].

Die Vorgehensweise bei der Auswertung der quasi-statischen Versuche ist in Bild 4.8 dargestellt. Die zugehörigen Last-Verformungskurven bestehen jeweils aus einem Hin- und einem Rückweg, wobei zwar ein elastisches Verformungsverhalten zu beobachten ist, die Rückdeformation jedoch zeitverzögert eintritt, so dass das Materialverhalten als visko-elastisch bezeichnet werden kann.

Nachdem ein Bereich identifizierte wurde, für den näherungsweise ein elastischer Last-Verformungs-Zusammenhang angenommen werden konnte (z.B. von 20 bis $80 \mathrm{kN}$ in Bild 4.8 wurden die zugehörigen Verformungsgrößen für Anfangs- bzw. Endbelastung (20 bzw. $80 \mathrm{kN}$ in Bild 4.8 abgelesen und die entsprechenden Mittelwerte für Hin- und Rückweg gebildet. Damit wurden zwei Punkte im Diagramm bestimmt: Anfangsbelastung mit zugehörigem Mittelwert der Verformungsgröße und Endbelastung mit zugehörigem Mittelwert. Die Steigung der Verbindungslinie dieser beiden Punkte ist ein Maß für die jeweilige Systemsteifigkeit. Dementsprechend wurden die in Bild 4.8 eingetragenen $\Delta$ Werte von Verformungen und Beanspruchungen zur Berechnung der Steifigkeitsparameter nach Gleichungen (4-2), (4-4) und (4-5) angesetzt.

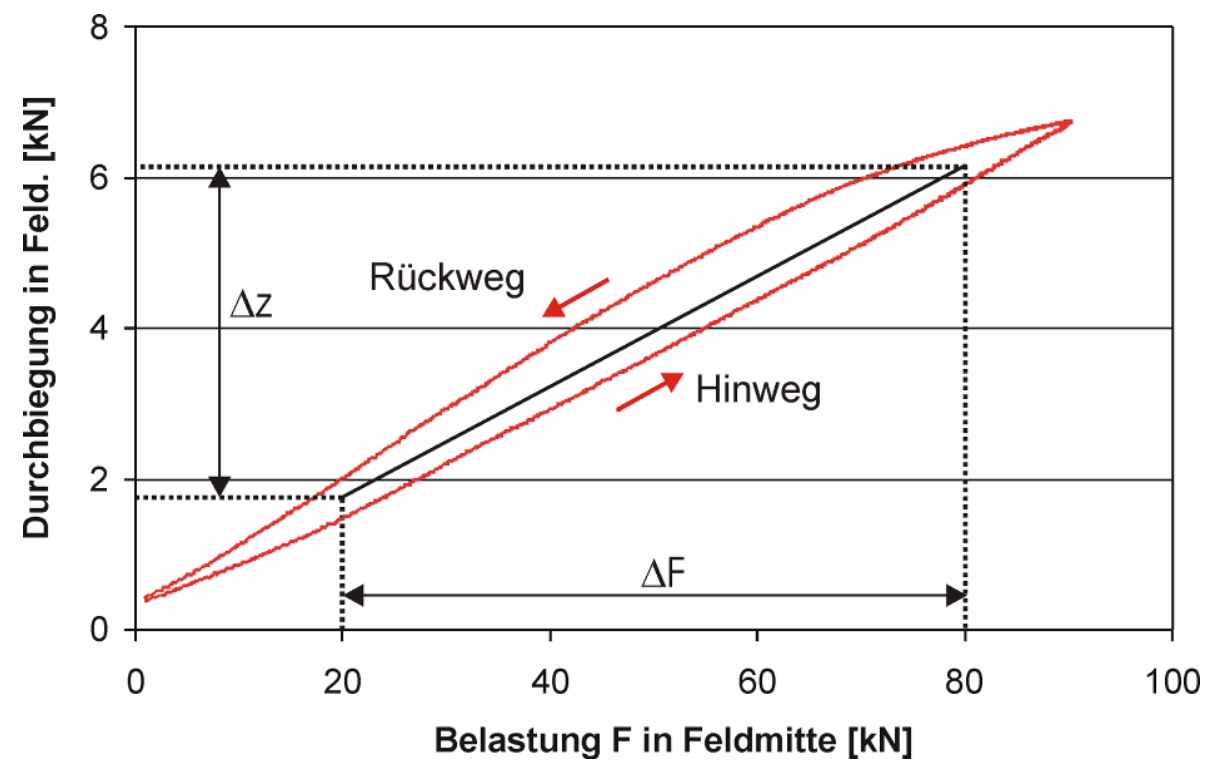

\section{Bild 4.8: Vorgehen bei der Auswertung}

Zur Auswertung der dynamischen Versuche wurde ein identischer Ansatz gewählt, wobei zusätzlich die Massenträgheitskräfte bei Aufstellung der Bestim- 
mungsgleichungen (4-2), (4-4) und (4-5) zu berücksichtigen waren. Diese haben insbesondere bei den höheren Testfrequenzen einen signifikanten Einfluss auf das dynamische Systemverhalten. Zur Berechnung des Steifigkeitsparameters $\mathrm{C}_{\mathrm{b} 2}$ wurde Gleichung (4-4) wie folgt erweitert:

$$
c_{b 2}=\frac{F-M \cdot a}{z_{1}+z_{2}}
$$

wobei a den Mittelwert der vertikalen Beschleunigung in Feldmitte und $\mathrm{M}$ die dynamisch wirksame Masse bezeichnen. Unter der Annahme, dass in Bezug auf die Biegebeanspruchung vornehmlich die erste Eigenform des Prüfkörpers angeregt wird, kann die wirksame Masse $\mathrm{M}$ mit

$$
M=\frac{m \cdot L}{2}
$$

angenähert werden, wobei $\mathrm{m}$ die (konstante) Masse je laufenden Meter bezeichnet.

Gleichung (4-7) gilt für diskrete Zeitpunkte. Allerdings wurde der Einfluss der Dämpfung nicht in Ansatz gebracht, so dass die Bestimmungsgleichung nur für Zeitpunkte gültig ist, wenn die Dämpfungskraft annähernd verschwindet. Dies ist unter Annahme visko-elastischer Dämpfungseigenschaften immer dann der Fall, wenn Verformung und Beschleunigung maximal und Geschwindigkeit minimal sind. Diese Zeitpunkte wurden für die Auswertung ausgewählt.

Wird eine sinusförmige Last mit der Frequenz $\Omega$ aufgebracht, so antwortet das System ebenfalls sinusförmig mit der Frequenz $\Omega$. Unter Berücksichtigung einer beliebigen Amplitude $\hat{z}$ und einem Phasenversatz $\alpha$ können der Zeitverlauf einer Verformungsgröße $z$ und die zugehörige Beschleunigung a folgendermaßen ausgedrückt werden:

$$
\begin{aligned}
& z=\hat{z} \cdot \sin (\Omega \cdot t-\alpha) \\
& a=-\hat{z} \cdot \Omega^{2} \cdot \sin (\Omega \cdot t-\alpha)
\end{aligned}
$$

Daraus ergibt sich

$$
a=-\Omega^{2} \cdot Z
$$

Mit diesem Zusammenhang zwischen $z$ und a sowie mit

$$
z_{\text {mean }}=\frac{1}{2} \cdot\left(z_{1}+z_{2}\right)
$$


kann Gleichung (4-7) wie folgt umgeformt werden:

$$
c_{b 2}=\frac{F-M \cdot a}{2 \cdot z_{\text {mean }}}=\frac{1}{2}\left(\frac{F}{Z_{\text {mean }}}+M \cdot \Omega^{2} \cdot z_{\text {mean }}\right)
$$

Gleichung (4-13) verdeutlicht den Einfluss der Masse M (geht linear ein) und der Belastungsfrequenz $\Omega$ (geht quadratisch ein) bei Bestimmung von $\mathrm{c}_{\mathrm{b} 2}$.

Zur Bestimmung der Koppelsteifigkeit waren ebenfalls die Einflüsse aus Massenträgheit zu berücksichtigen, so dass Gleichung (4-5) entsprechend nachfolgender Gleichung erweitert wurde:

$$
c_{\text {coup }}=\frac{F-\frac{M}{2} \cdot a-c_{b 2} \cdot z_{1}}{z_{1}-e \cdot \varphi}
$$

Dabei wurde nur die Hälfte der in Gleichung (4-7) angesetzten Masse in Ansatz gebracht, da sich bei Testmodus M3 die eine Kastenhälfte in Ruhe befindet. Gleichung (4-14) lässt sich analog zu Gleichung (4-13) weiter umformen, indem die Beschleunigung a mittels entsprechender Verformungsgröße substituiert wird.

Die Versuche wurden mit relativ kleinen Wegamplituden und niedrigen Frequenzen durchgeführt. Signifikante Setzungen des Schotters, die aus einer Destabilisierung des Korngerüsts resultieren können und insbesondere bei hohen Beschleunigungs- und Wegamplituden auftreten [1] [68] [70], wurden nach dem Verdichten vor Versuchsbeginn im Verlaufe der Versuche nicht mehr festgestellt.

\subsection{Versuchsergebnisse}

In Bild 4.9 sind die Ergebnisse der quasi-statischen Tests dargestellt. Für die Testreihen S1-S4 sind jeweils die ermittelten Werte $c_{b 1}-C_{b 3}$ (linkes Diagramm) und $\mathrm{c}_{\text {coup }}$ (rechtes Diagramm) gegeben. Für den Steifigkeitsparameter $\mathrm{c}_{\mathrm{b} 1}$ (leerer Kasten) wurde ein Wert von etwa $90 \mathrm{kN} / \mathrm{cm}$ ermittelt. Daraus ergibt sich unter Verwendung von Gleichung (4-3) eine Biegesteifigkeit $\mathrm{El}=12900 \mathrm{kNm}^{2}$, die gut mit dem berechneten Wert von $\mathrm{EI}=13490 \mathrm{kNm}^{2}$ übereinstimmt (siehe oben).

Durch Vergleich der Steifigkeitsparameter $c_{b 1}$ und $c_{b 3}$ kann der Einfluss des Schotters auf die Biegesteifigkeit des Prüfkörpers beurteilt werden. Die Ergebnisse zeigen einen Anstieg der Steifigkeit um etwa $5 \%$. Allerdings ist hierbei zu berücksichtigen, dass ein Großteil des Schotters bei Durchführung von Testmodus M4 unbelastet war, was deutliche Auswirkungen auf die Höhe des Steifig- 
keitsbeitrags haben dürfte. In dieser Hinsicht unterschieden sich die Randbedingungen im Versuch von der Situation auf belasteten Eisenbahnbrücken durch die lastverteilende Wirkung der Schwellen kann bei Zugüberfahrten von einem gleichmäßig belasteten Schotterbett ausgegangen werden.

a)

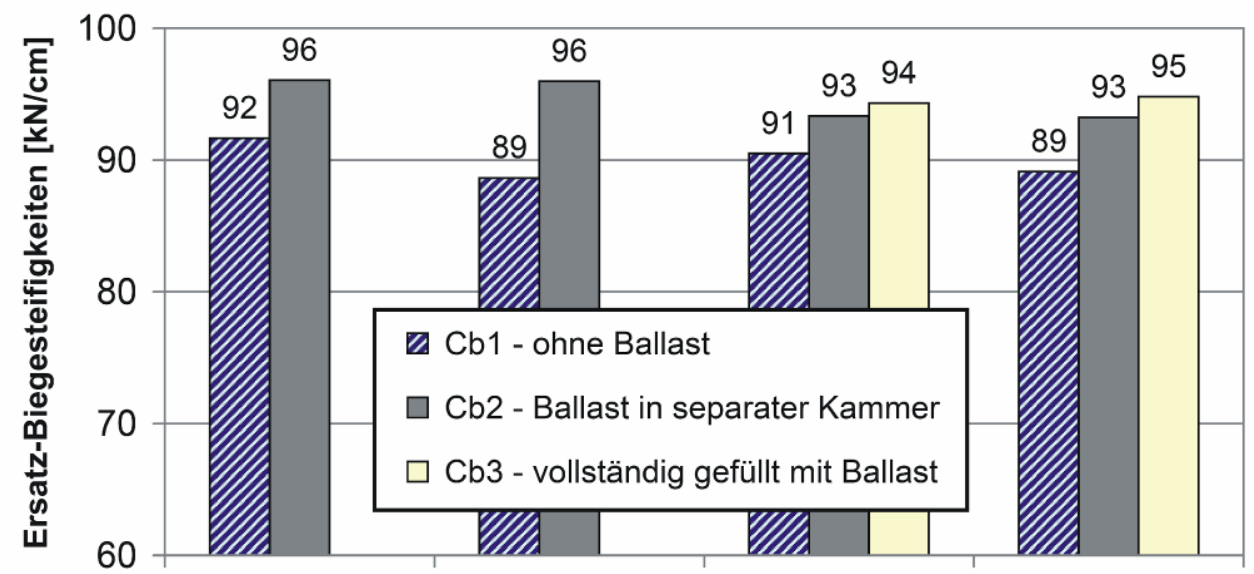
S1
S2
S3
S4

b)

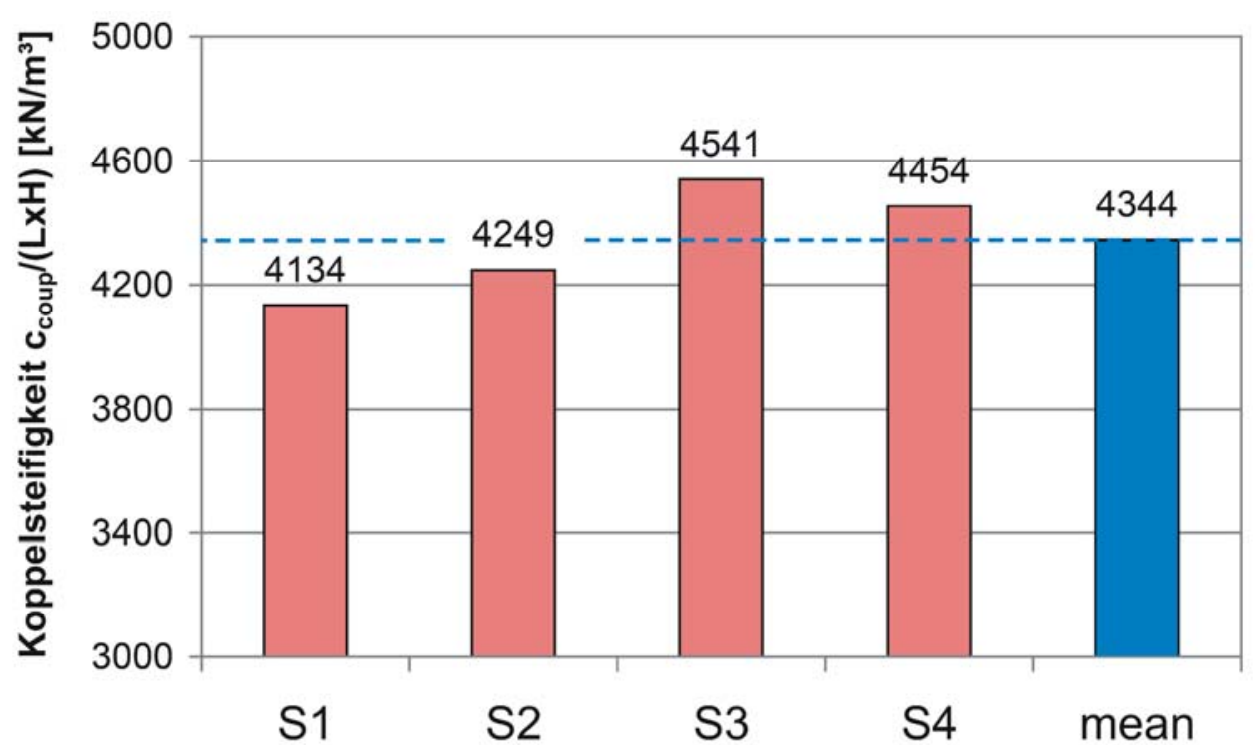

Bild 4.9: Ergebnisse aus den quasi-statischen Versuchen für a) die Steifigkeitsparameter $c_{b 1}-c_{b 3}$ sowie b) $c_{\text {coup }}$ (Auswertung der Versuchsreihen S1-S4)

Für die Koppelsteifigkeit, mit welcher der Lastübertrag von einer Kastenhälfte in die andere abgebildet wurde, ergaben sich für die vier Versuchsreihen Werte zwischen 4100 bis $4600 \mathrm{kN} / \mathrm{m}^{3}$ mit einem Mittelwert von etwa $4350 \mathrm{kN} / \mathrm{m}^{3}$. Der ermittelte Parameter $\mathrm{c}_{\text {coup }}$ wurde dabei durch die Größe der Kontaktfläche zwischen den beiden Kastenhälften geteilt, um eine spätere Verwendung in numerischen Modellen zu ermöglichen. Unter der Annahme eines proportionalen Zusammenhangs zwischen Kontaktfläche und Koppelsteifigkeit lassen sich damit die Steifigkeiten der Ersatzfedern zur Abbildung des Koppeleffekts in Abhängig- 
keit vom Abstand der Federelemente und der Höhe der Schotterschicht sehr einfach bestimmen, vgl. Berechnungsbeispiel in Abschnitt 7.

Als aufschlussreich im Hinblick auf die dynamischen Eigenschaften des vollständig mit Schotter gefüllten Kastens erwies sich die Untersuchung von Abklingkurven, die mit Hilfe von Hammerschlägen erzeugt wurden. Ein Beispiel für solch eine Kurve ist in Bild 4.10 dargestellt, wobei das Signal eines Beschleunigungssensors in Feldmitte wiedergegeben ist. Zur Eliminierung unrelevanter Frequenzanteile wurde ein $50 \mathrm{~Hz}$ Tiefpassfilter verwendet.

Durch den Hammerschlag wurde das System hauptsächlich in der ersten Biegeeigenfrequenz von etwa $16 \mathrm{~Hz}$ angeregt. Im Zeitraum zwischen 10,5 s und $11,0 \mathrm{~s}$ wurden acht Schwingungsperioden registriert, woraus sich eine Schwingfrequenz von $16,0 \mathrm{~Hz}$ ergibt. Betrachtet man hingegen den Zeitraum zwischen $10,0 \mathrm{~s}$ und $10,5 \mathrm{~s}$ mit deutlich höheren Schwingungsamplituden, so lassen sich nur 7,5 Perioden zählen, woraus sich eine niedrigere Eigenfrequenz von $15 \mathrm{~Hz}$ berechnet. Offensichtlich hat die Höhe der Schwingungsamplituden Einfluss auf die Systemsteifigkeit, wobei sich mit höheren Amplituden geringere Steifigkeiten einstellen. Dieser Effekt wurde an anderer Stelle der Versuchsauswertung ebenfalls festgestellt, vgl. Bild 4.11. Er wurde bereits in [30] [31] [51] [53] beschrieben und lässt sich durch einen nicht-linearen Steifigkeitseinfluss des Schotters erklären. Größere Schwingungsamplituden des Prüfkörpers können zu einer Instabilität der Schotterschicht führen, was eine geringere Steifigkeit zur Folge hat.

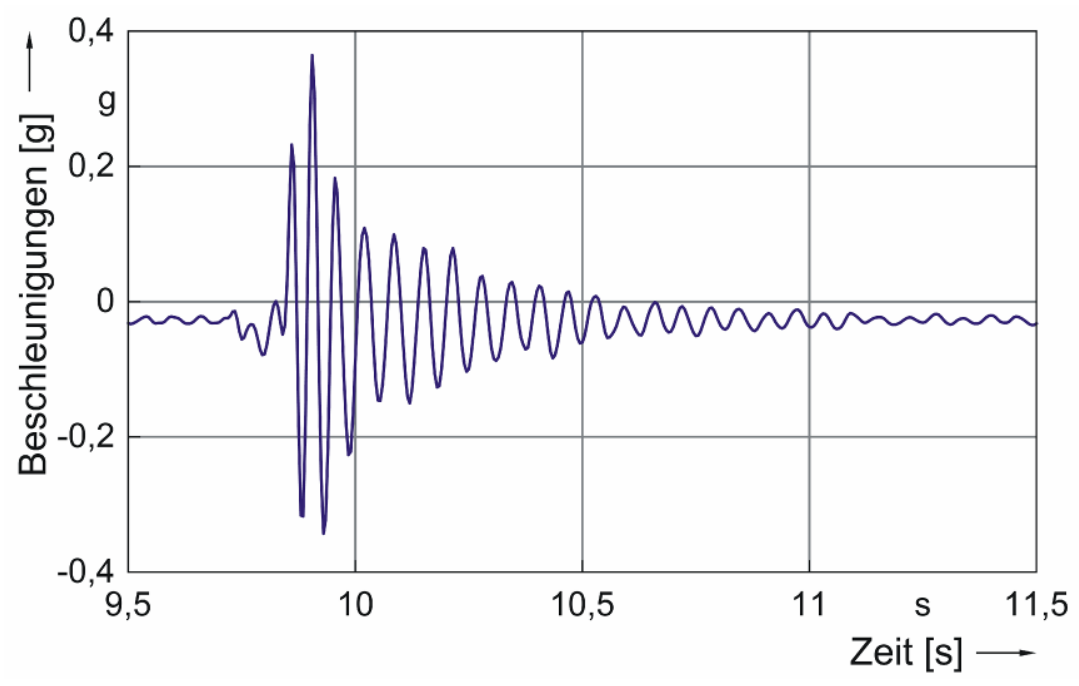

Bild 4.10: Abklingkurve nach einem Hammerschlag auf den vollständig mit Schotter gefüllten Kasten (Beschleunigungen)

Im Hinblick auf die Dämpfungseigenschaften wurde ebenfalls ein nicht-lineares Verhalten beobachtet. Für den Beginn des Abklingprozesses, siehe Bild 4.10, 
wurde unter Verwendung der Gleichungen (3-10) und (3-12) ein Wert für die Lehr'sche Dämpfung von etwa $5 \%$ abgeleitet. Im Anschluss an die ersten hohen Schwingungsamplituden wurde dann allerdings ein deutlich niedrigerer Wert von etwa $3 \%$ festgestellt. Ausschlaggebend für diesen Effekt der amplitudenabhängigen Dämpfung dürften, wie bereits in Abschnitt 2.5 erörtert, Reibungsvorgänge zwischen den einzelnen Schottersteinen sein, die sich bei hohen Amplituden verstärkt einstellen und die Systemdämpfung signifikant vergrößern.

a)

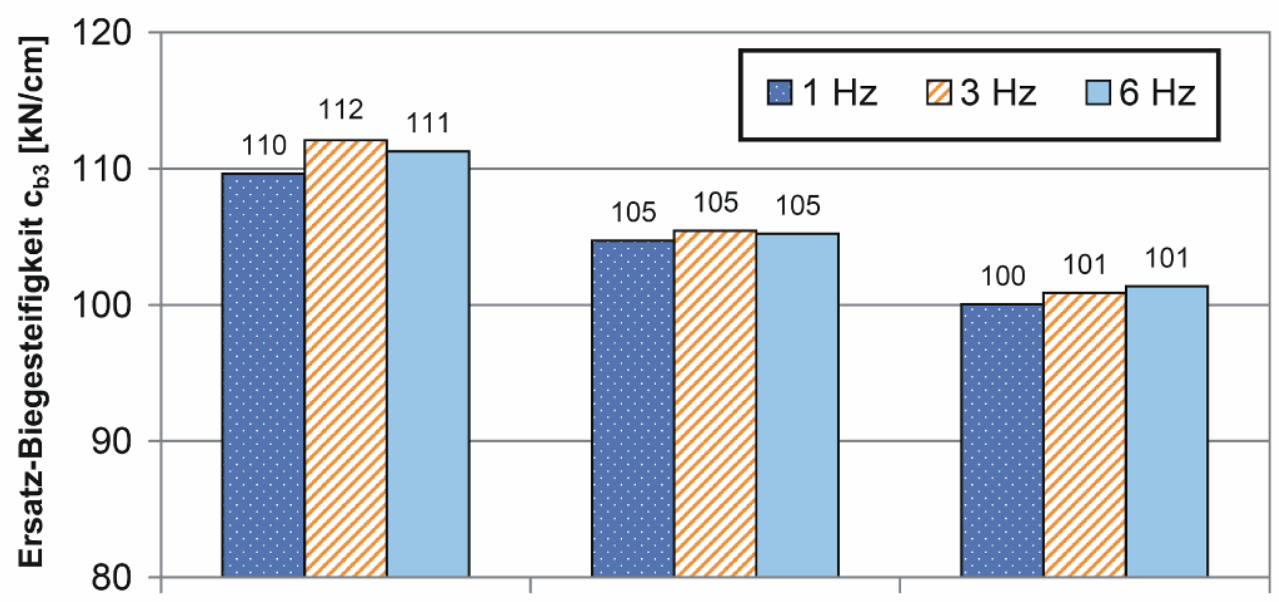

$$
\mathrm{F}= \pm 5 \mathrm{kN} \quad \mathrm{F}= \pm 10 \mathrm{kN} \quad \mathrm{F}= \pm 20 \mathrm{kN}
$$

b)

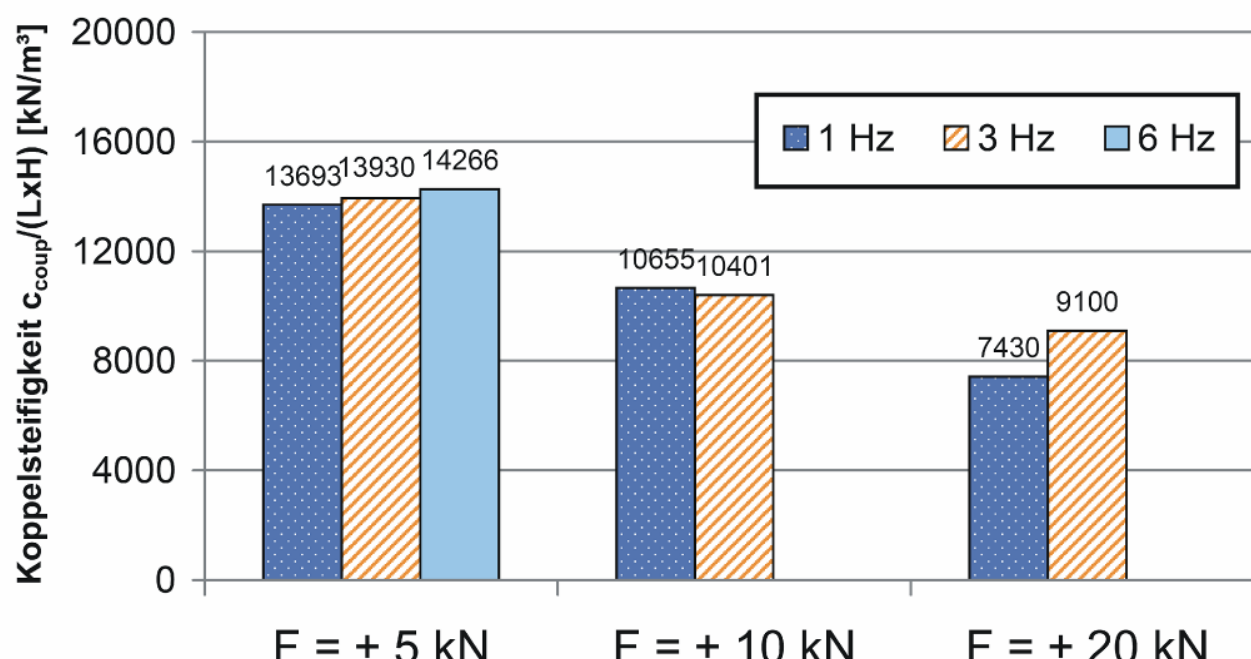

Bild 4.11: Ergebnisse aus den dynamischen Versuchen für a) den Steifigkeitsparameter $c_{b 3}$ sowie b) $c_{\text {coup }}$ (Mittelwerte aus allen Versuchsreihen)

Bild 4.11 zeigt die Ergebnisse der dynamischen Schwingversuche, wobei hier jeweils die Mittelwerte aus den vier Testserien S1 bis S4 gegeben sind. Im Diagramm auf der linken Seite ist der Parameter $c_{b 3}$ in Abhängigkeit vom Lastniveau und der Anregungsfrequenz aufgetragen, im Diagramm auf der rechten Seite der Parameter $\mathrm{c}_{\text {coup }}$, wobei hier die Lastfrequenz von $6 \mathrm{~Hz}$ betriebsbedingt 
nur für die kleinen Amplituden angesteuert wurde. Für den gewählten Frequenzbereich hat die Belastungsfrequenz keinen signifikanten Einfluss auf die Ergebnisse, während sich das Lastniveau deutlich auf die Steifigkeitseigenschaften auswirkt. Die höchsten Steifigkeitswerte wurden bei kleinen Last- bzw. Verformungsamplituden festgestellt. Der Wert für $\mathrm{c}_{\mathrm{b} 3}$ sinkt von $110 \mathrm{kN} / \mathrm{cm}$ bei einem Lastniveau von $\pm 5 \mathrm{kN}$ auf $100 \mathrm{kN} / \mathrm{cm}$ bei einem Lastniveau von $\pm 20 \mathrm{kN}$. Verglichen mit dem Resultat der quasi-statischen Versuche von $95 \mathrm{kN} / \mathrm{cm}$ (Bild 4.9), wurde also eine dynamische Steifigkeit ermittelt, die $15 \%$ bzw. $5 \%$ höher ist.

Im Hinblick auf den Parameter $\mathrm{c}_{\text {coup }}$ erwies sich die Abhängigkeit vom Lastniveau als noch auffälliger. Für kleine Lastamplituden von $\pm 5 \mathrm{kN}$ wurde mit einem Wert von rund $14.000 \mathrm{kN} / \mathrm{m}^{3}$ eine dreimal höhere Steifigkeit gemessen als bei den quasi-statischen Versuchen, bei denen sich ein Mittelwert von $4350 \mathrm{kN} / \mathrm{m}^{3}$ ergab. Bei größeren Lastamplituden reduzierte sich der Steifigkeitswert deutlich - auf $8260 \mathrm{kN} / \mathrm{m}^{3}$ bei $\pm 20 \mathrm{kN}$ (Mittelwert der 1,0 and 3,0 Hz Versuche). Allerdings ist dieser Wert immer noch deutlich größer als der aus den quasistatischen Versuchen abgeleitete Steifigkeitsparameter von $4350 \mathrm{kN} / \mathrm{m}^{3}$, der damit einen unteren Grenzwert für die Koppelsteifigkeit darstellt.

Die Steifigkeitsabnahme bei großen Amplituden wurde bereits bei der Analyse von Abklingkurven nach Stoßanregungen des Systems festgestellt, vgl. Erläuterungen zu Bild 4.10. Mit der Auswertung der dynamischen Versuche wurde dieser Effekt bestätigt. Es handelt sich offenbar um ein charakteristisches Merkmal des Schotters, dass sein Steifigkeitsbeitrag von der Größe der Schwingungsamplituden abhängt, vgl. auch Abschnitte 3.2.6 und 3.2.7. Diese Eigenschaft wurde insbesondere im Falle der Koppelsteifigkeit beobachtet.

Es ist darauf hinzuweisen, dass im Rahmen der Versuche nur ein begrenzter Lastbereich untersucht wurde, da die Maximallast des Prüfzylinders $100 \mathrm{kN}$ betrug. Für diesen Lastbereich wurden ausschließlich lineare Zusammenhänge zwischen Last- und Verformungsgrößen festgestellt. Zweifelsfrei würden sich bei Aufbringung deutlich höherer Lasten nicht-lineare Effekte bemerkbar machen, die auf ein "Durchrutschen“ in der Koppelfuge schließen ließen. Denn ab einer bestimmten Kontaktkraft zwischen den einzelnen Schottersteinen würde die Haftreibung, die für ein stabiles Gefüge im Schotter sorgt, überwunden, so dass sich die Steine bei Vorhandensein von Gleitreibungskräften gegeneinander verschieben könnten.

Dieser „Durchrutscheffekt“ wird im Eurocode 1 [10] bzw. DIN-Fachbericht 101 [11] berücksichtigt. Dort ist in Abbildung K.3 der Zusammenhang zwischen 
Längsverschiebewiderstand des Gleises und der horizontalen Relativverschiebung des Gleises gegenüber dem Brückendeck gegeben, vgl. Bild 4.12c. Dieses Last-Verformungs-Verhalten ist zu berücksichtigen, falls horizontale Beanspruchungen wie Brems- und Anfahrlasten oder auch Temperaturlasten über das Schotterbett in den Unterbau bzw. Unterrund weitergeleitet werden sollen. Das in Bild 4.12c dargestellte Diagramm gibt einen Verformungsgrenzwert vor, der den Übergang von elastischem zu inelastischem Verhalten darstellt. Bis zu einer Relativverschiebung von 2,0 mm wird ein elastischer Zusammenhang angenommen, für größere Verformungen ist ein "Durchrutschen“ ohne weitere Laststeigerung anzunehmen. Während der Versuche konnte solch ein Effekt nicht festgestellt werden, obwohl bei den quasi-statischen Versuchen Relativverschiebungen in der Koppelfuge von bis zu 6,0 mm gemessen wurden, vgl. Tabelle 4.2. Nach Ansicht des Verfassers widersprechen diese Ergebnisse jedoch nicht zwangsläufig dem normativen vorgegebenen Grenzwert von 2,0 mm, zumal bei den dynamischen Versuchen nur maximale Relativverschiebungen von 2,2 mm gemessen wurden. Es ist durchaus denkbar, dass höhere Frequenzanteile, höhere Beanspruchungen sowie Stoßeffekte, die bei Zugüberfahrten zu erwarten sind, einen Durchrutscheffekt begünstigen und somit den aus experimenteller Sicht relativ niedrigen normativen Grenzwert von 2,0 mm rechtfertigen. Diese Überlegungen spielten eine wichtige Rolle im Hinblick auf die weitere Verwendung der experimentellen Ergebnisse, vgl. Abschnitt 4.5.

Tabelle 4.2: Gemessene Relativverschiebungen während Testmodus M3 (Maximalwerte aus allen Testreihen)

Maximale Relativverscheibungen im Versuch [mm]

\begin{tabular}{cccc}
\hline \multicolumn{2}{c}{ Dynamische Versuche } & $\begin{array}{c}\text { Quasi-statische } \\
\text { Versuche } \\
90 \mathrm{kN} \text { max }\end{array}$ \\
\hline $\mathrm{F}= \pm 5 \mathrm{kN}$ & $\mathrm{F}= \pm 10 \mathrm{kN}$ & $\mathrm{F}= \pm 20 \mathrm{kN}$ & 5,8 \\
\hline 0,4 & 1,0 & 2,2 & 5 \\
\hline
\end{tabular}

In diesem Zusammenhang ist noch zu erwähnen, dass sich im Fugenbereich von Brücken mit Schotteroberbau an den Übergängen Brücke-Widerlager erhebliche Abrieberscheinungen an den Schottersteinen einstellen können, die zu den sogenannten „weißen Stellen“ führen [68]. Zurückzuführen sind die „weißen Stellen" auf das weiße Gesteinsmehl, das beim Abrieb der Schottersteine entsteht und auf dem Schotterbett sichtbar wird. Dieser Effekt kann sich auch im Bereich der Bauwerksfuge bei baulich getrennten eingleisigen Brückenüberbauten mit durchgehendem Schotterbett bemerkbar machen. Zu erkennen ist der 
Abrieb an hellen Streifen im Schotterbett entlang der Fuge. Oft geht diese Erscheinung einher mit einer leichten Muldenbildung in selbigem Bereich.

In [1] [22] wird erörtert, dass sich die Alterung von Eisenbahnschotter auf dessen Scherfestigkeit und Deformationsverhalten auswirkt. Während bei frisch gebrochenem Schotter eine stabile Verzahnung der einzelnen Gesteinskörner sichergestellt ist, treten mit zunehmender Nutzungsdauer Abplatzungen und Abriebseffekte auf, die in Verbindung mit einem fortschreitenden Verschmutzungsgrad dazu führen können, dass die Gesteinskörner leichter aneinander vorbeigleiten. Die Folge sind Umlagerungen im Schotterbett, die sich in Setzungen ausdrücken. Eine fortschreitende Schädigung kann dann zu Gleislageungenauigkeiten führen, auf die in der Regel mit dem Einsatz einer Stopfmaschine und gegebenenfalls mit einer Erneuerung des Schotterbetts reagiert wird.

Auch wenn im Verlaufe der Versuche eine Abnutzung der Schottersteine festgestellt wurde, so waren die Anzahl der aufgebrachten Lastzyklen sowie die einwirkende Belastung zu gering, um einen mit realen Bedingungen vergleichbaren Schotterabrieb hervorzurufen. Die ermittelten Werte gelten also ausdrücklich für einen neuwertigen Schotter. Ein gebrauchter, abgenutzter Schotter wurde im Rahmen der Versuche nicht getestet. Aus vorgenannten Gründen ist es aber durchaus möglich, dass bei Verwendung eines gebrauchten bzw. gealterten Schotters das oben behandelte „Durchrutschen“ in der Koppelfuge hätte registriert werden können.

\subsection{Verwendung der experimentellen Ergebnisse in nume- rischen Berechnungen}

Es ist bereits allgemein üblich, Feder- und Dämpferelemente zur Modellierung von Schotterschichten auf Eisenbahnbrücken zu verwenden. In Bild 4.12a ist ein typisches 2D-Modell eines Einfeld-Systems dargestellt - allerdings ohne Dämpferelemente, die in gleicher Weise wie die Federelemente angeordnet werden können. In der Regel wird die Dämpfung jedoch mit Hilfe einer global wirksamen Systemdämpfung berücksichtigt. Detaillierte Beschreibungen verschiedener Modellierungsvarianten sind beispielsweise in [20] [53] [56] [67] zu finden.

Während auf die in Bild 4.12a dargestellten Federn in vertikaler Richtung hier nicht näher eingegangen werden soll, bilden die eingezeichneten horizontalen Federelemente einen ähnlichen Effekt ab wie den im Rahmen der Ballastversuche untersuchten Koppeleffekt. In beiden Fällen findet eine Schubkraftübertragung über die Schotterschicht statt, die durch Reibkräfte zwischen den einzel- 
nen Schottersteinen ermöglicht wird. Auf der einen Seite werden Horizontalkräfte zwischen Gleis und Brückendeck übertragen (Bild 4.12a), auf der anderen Seite Vertikalkräfte zwischen baulich getrennten Überbauten, vgl. hierzu Bild 4.2 .

a)

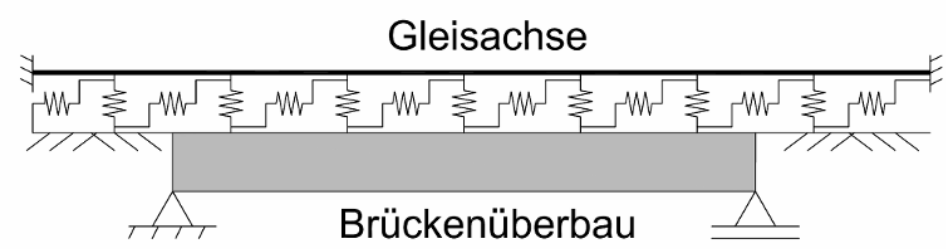

b)

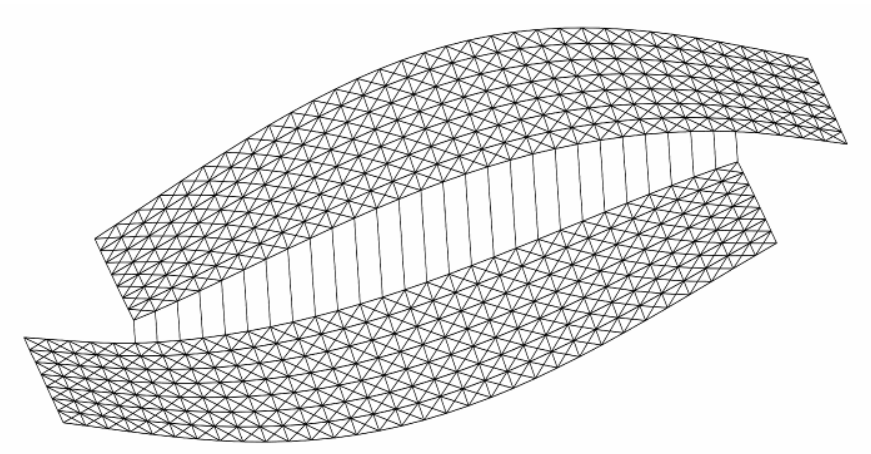

c)

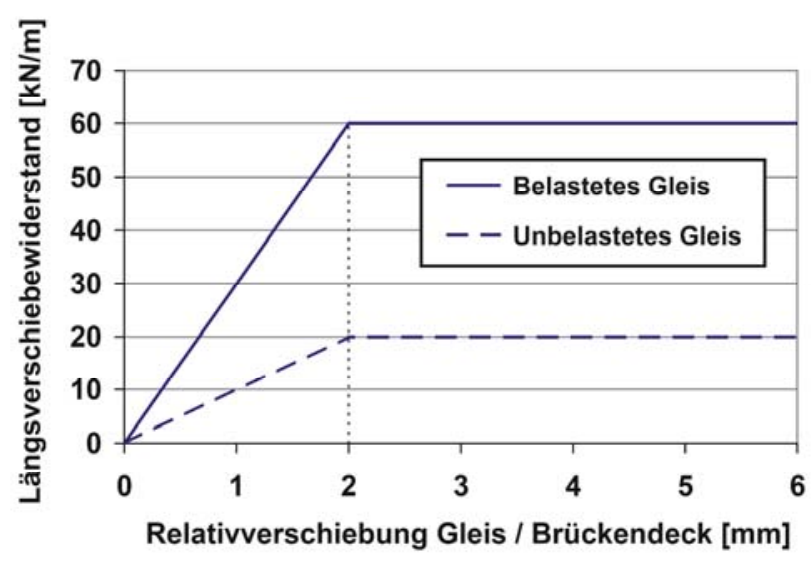

Bild 4.12: a) 2D-Modell mit Federelementen zur Berücksichtigung des Schotteroberbaus b) 3D-Modell mit Federelementen zur Berücksichtigung der Koppelwirkung durch den Schotter (Darstellung 2. Eigenform) c) Längsverschiebewiderstand in Abhängigkeit der horizontalen Relativverschiebung zwischen Gleis und Brückendeck nach DIN-Fachbericht 101 [11]

Vor diesem Hintergrund wurde ein analoges Vorgehen zur Modellierung des Koppeleffekts in numerischen Modellen gewählt - die Verwendung von Federelementen, welche die beiden Überbauten im Bereich der Koppelfuge miteinander verbinden, vgl. Bild 4.12b. Diese Herangehensweise erfordert ein gegenüber dem zweidimensionalen Modell deutlich komplexeres 3D-Modell, dessen Berechnung zeitintensiver ist und beim Anwender ein hohes Maß an Sach- 
kenntnis auf dem Gebiet der Brückenmodellierung voraussetzt - ein Aspekt, der in Abschnitt 5.5 bei der Diskussion eines vereinfachten Tragwerkmodells zur Berücksichtigung des Koppeleffekts aufgegriffen wird.

Tabelle 4.3: Gegenüberstellung von Steifigkeitsansätzen für die Kraftübertragung durch das Schotterbett

\begin{tabular}{lr}
\hline \multicolumn{1}{c}{ Vergleich Steifigkeitseigenschaften (bezogen auf Kontaktfläche) } \\
\hline Steifigkeit horizontal (belastet) - elastischer Bereich & $10.000 \mathrm{kN} / \mathrm{m}^{3}$ \\
Steifigkeit horizontal (unbelastet) - elastischer Bereich & $3.330 \mathrm{kN} / \mathrm{m}^{3}$ \\
$\begin{array}{l}\text { Steifigkeit vertikal - Kopplungseffekt Ballast } \\
\text { (aus Versuchen abgeleiteter Wert) }\end{array}$ & $4.350 \mathrm{kN} / \mathrm{m}^{3}$ \\
\hline
\end{tabular}

Das in Bild 4.12c wiedergegebene Last-Verformungs-Diagramm aus dem DINFachbericht 101 [11], das bereits im vorangegangenen Abschnitt erörtert wurde, lässt sich dazu heranziehen, Steifigkeiten für die horizontalen Federelemente aus Bild 4.12a zu berechnen, die Horizontalkräfte in Längsrichtung zwischen Gleis und Brückendeck übertragen. Nimmt man auf Grundlage von [11] eine Lastverteilungsbreite unter den Gleisen von 3,0 m an, so ergeben sich für ein belastetes bzw. ein unbelastetes Gleis im elastischen Bereich die in Tabelle 4.3 angegebenen Steifigkeitswerte, bezogen auf die Kontaktfläche in der Koppelfuge. Der erste Wert in der Tabelle berechnet sich demnach wie folgt:

$$
\frac{60 \mathrm{kN} / \mathrm{m}}{2,0 \mathrm{~mm} \cdot 3,0 \mathrm{~m}}=10.000 \mathrm{kN} / \mathrm{m}^{3}
$$

Den aus der Norm abgeleiteten Steifigkeitswerten wird in Tabelle 4.3 der Mittelwert gegenübergestellt, der sich bei den experimentellen Untersuchungen als unterer Grenzwert der Koppelsteifigkeit ergab, vgl. Abschnitt 4.4. Es ist plausibel, dass dieser Wert von $4350 \mathrm{kN} / \mathrm{m}^{3}$ deutlich kleiner ist als der Wert von $10000 \mathrm{kN} / \mathrm{m}^{3}$ für die horizontale Steifigkeit bei belastetem Gleis, da im Versuch kein vergleichbarer Anpressdruck in der Kontaktfläche zwischen den beiden Kastenhälften vorhanden war. Andererseits war ein gewisser Anpressdruck durchaus vorhanden, resultierend aus dem Horizontalschub, der ein seitliches Abstützen des Versuchskörpers erforderlich machte. Somit ist auch plausibel, dass mit dem Wert von $4350 \mathrm{kN} / \mathrm{m}^{3}$ der in Tabelle 4.3 angegebene Wert von $3330 \mathrm{kN} / \mathrm{m}^{3}$ für die horizontale Steifigkeit bei unbelastetem Gleis etwas überschritten wird. 
Der experimentell ermittelte Wert von $4350 \mathrm{kN} / \mathrm{m}^{3}$ ist also sehr gut im Einklang mit den normativ vorgegebenen Längsverschiebewiderständen zum Abtrag von horizontalen Beanspruchungen. Aufgrund der Versuchsergebnisse ist zwar zu erwarten, dass die dynamische Koppelsteifigkeit bei kleinen Last- bzw. Verformungsamplituden größer ist als dieser Wert, jedoch treten bei bemessungsrelevanten Zugüberfahrten relativ hohe Lasten bzw. Verformungen in der Koppelfuge auf, so dass der Ansatz einer Koppelsteifigkeit größer $4350 \mathrm{kN} / \mathrm{m}^{3}$ nicht zu rechtfertigen ist. Es ergäbe sich sonst die Gefahr, dass damit die Systemsteifigkeit überschätzt würde und auf der unsicheren Seite liegende numerische Ergebnisse erzeugt würden.

Vor diesem Hintergrund wird es als sinnvoll erachtet, den unteren Grenzwert für die Koppelsteifigkeit von $4350 \mathrm{kN} / \mathrm{m}^{3}$ zur Definition der Koppelfedern im numerischen Modell zu verwenden. Allerdings kann nicht ausgeschlossen werden, dass die tatsächlich vorhandene Koppelsteifigkeit bei WIB-Überbauten mit diesem Wert nur ungenau beschrieben wird. Folgende Faktoren können hierfür ursächlich sein.

- Beschaffenheit des Schotters abweichend von den Randbedingungen im Versuch (Gesteinsart, Körnung, Bruchbild, Abnutzungsgrad, Verschmutzungsgrad, Feuchte- und Klimaeinfluss)

- Ungenauigkeit der mechanischen Modelle, die zur Auswertung verwendet wurden

- Messungenauigkeiten bei Durchführung der Versuche

Um zu untersuchen, wie sich eine etwaige Ungenauigkeit der ermittelten Koppelsteifigkeit auf die numerische Ergebnisse von Überfahrtsimulationen auswirkt, wurden Parameterstudien durchgeführt, die in Abschnitt 5.5.2 behandelt werden.

Zu klären ist nun noch die Frage, was für ein Federgesetz bei der Implementierung von Federelementen zur Abbildung des Schottereffekts berücksichtigt werden sollte. Im vorangegangenen Abschnitt wurde bereits ausgeführt, dass ein „Durchrutschen" während der Versuche zwar nicht beobachtet wurde, dieser Effekt aber in Realität keineswegs ausgeschlossen werden kann - insbesondere vor dem Hintergrund, dass für die Versuche neuwertiger Schotter verwendet wurde, der Abnutzungsgrad des „Altschotters“ auf Brücken jedoch erheblich sein kann. In Tabelle 4.4 sind für unterschiedliche Zugtypen Relativverformungen angegeben, die während Zugüberfahrten gemessen wurden. 
Tabelle 4.4: Gemessene Relativverschiebungen in der Koppelfuge während Zugüberfahrten (Mittelwerte aus jeweils zehn Überfahrten je Zugtyp)

Maximale Relativverscheibungen bei Zugüberfahrten [mm]

\begin{tabular}{cccc}
\hline ICE 3 & Thalys & RE & Güterzüge \\
\hline$v=250 \mathrm{~km} / \mathrm{h}$ & $\mathrm{v}=200 \mathrm{~km} / \mathrm{h}$ & $\mathrm{v}=150 \mathrm{~km} / \mathrm{h}$ & $\mathrm{v}=100 \mathrm{~km} / \mathrm{h}$ \\
\hline $\mathbf{4 , 3}$ & $\mathbf{3 , 5}$ & $\mathbf{3 , 8}$ & $\mathbf{4 , 1}$ \\
\hline
\end{tabular}

Der Grenzwert von 2,0 mm, der gemäß Norm bei der Weiterleitung von horizontalen Beanspruchungen den Übergang von elastischem zu inelastischem Verhalten darstellt (vgl. Bild 4.12c), wird mit maximal knapp 4,5 mm deutlich überschritten. Damit bewegen sich die auftretenden Relativverformungen in einem Bereich, in dem in Anlehnung an die normativen Vorgaben bezüglich des horizontalen Längsverschiebewiderstandes mit einem „Durchrutschen“ zu rechnen ist. Zwar ließe sich für diesen Verformungsbereich vom Standpunkt der Versuche aus ein vollständig linear-elastischer Ansatz rechtfertigen (vgl. Relativverformungen während der Versuche in Tabelle 4.2), aber es bestünde bei dieser Systemidealisierung wiederum die Gefahr, zu viel Steifigkeit in das System einzubringen, so dass auf der unsicheren Seite liegende numerische Ergebnisse erzeugt würden.

Die Verwendung eines bilinearen Federgesetzes wird daher als sinnvoll angesehen, wobei der normative Grenzwert von 2,0 mm übernommen wird, bei dem ein Durchrutschen einsetzt. Das entsprechende Federgesetz zur Beschreibung des Widerstands in der Koppelfuge zeigt Bild 4.13 die Kraft, die in Abhängigkeit von der Relativverformung in der Koppelfuge aufgetragen ist, wird dabei auf die Kontaktfläche bezogen, die durch das entsprechende Federelement repräsentiert wird. Die Grenzkraft bei 2,0 mm Relativverformung berechnet sich wie folgt:

$$
F_{\text {grenz }}=4350 \mathrm{kN} / \mathrm{m}^{3} \cdot 0,002 \mathrm{~m}=8,7 \mathrm{kN} / \mathrm{m}^{2}
$$

Der Frage, wie stark sich die Wahl des Federgesetzes (linear oder bilinear) auf die Resultate von Überfahrtsimulationen auswirkt, wird in Abschnitt 5.5.2 ebenfalls im Rahmen von Parameterstudien nachgegangen. 


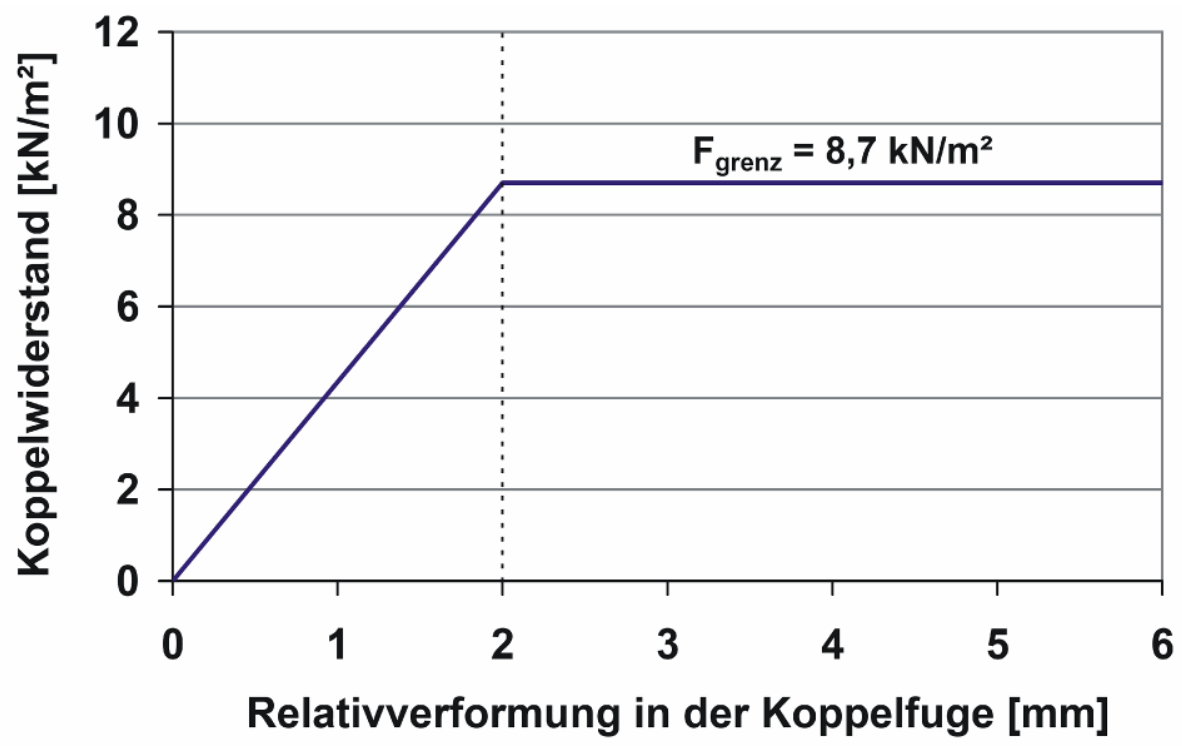

Bild 4.13: Koppelwiderstand (bezogen auf die Kontaktfläche zwischen den angrenzenden Schotterschichten) in Abhängigkeit der vertikalen Relativverschiebung in der Koppelfuge

Mit Hilfe der experimentellen Untersuchungen an Eisenbahnschotter wurde ein mechanisches Modell erarbeitet, um den durch Schotter verursachten Koppeleffekt in numerischen Berechnungen abzubilden. Es muss aber betont werden, dass es sich hierbei um ein vereinfachtes ingenieurmäßiges Modell handelt, das spezielle Effekte wie die beobachtete amplitudenabhängige Steifigkeitsänderung sowie das visko-elastische Verformungsverhalten nicht beinhaltet. Dennoch reicht es im Sinne eines Näherungsverfahrens aus, um im Rahmen von Simulationsrechnungen realistische Ergebnisse zu erzeugen, wie im nun folgenden Abschnitt u. a. gezeigt wird. 


\section{Numerische Untersuchungen an WIB-Brücken zum Ein- fluss baulicher Randbedingungen}

\subsection{Generelles Vorgehen}

Um den Einfluss der in Abschnitt 0 genannten baulichen Randbedingungen auf das dynamische Verhalten von WIB-Brücken quantitativ beurteilen zu können, wurden numerische Studien an den bereits messtechnisch untersuchten Überbauten (vgl. Tabelle 3.1) durchgeführt. Es wurden diejenigen Brücken ausgewählt, für die ausreichende Informationen hinsichtlich ihrer konstruktiven Durchbildung vorlagen, so dass die jeweiligen baulichen Randbedingungen wirklichkeitsnah abgebildet werden konnten.

Zunächst wurden wie bei einer herkömmlichen dynamischen Bemessung numerische Modelle erstellt, die nur den nackten Brückenquerschnitt darstellen - ohne Berücksichtigung zusätzlicher die Brückendynamik beeinflussender Effekte („Ausgangsmodelle“). Anschließend wurden die Modelle modifiziert, indem die einzelnen baulichen Randbedingungen in sinnvoller Weise numerisch abgebildet und hinzugefügt wurden. Im Rahmen von Parameterstudien wurde dann die Wirkung der Randbedingungen auf die dynamischen Brückeneigenschaften untersucht.

Als Bewertungskriterien dienten zum einen die modalen Systemeigenschaften der Brückenmodelle, zum anderen die zeitlichen Verläufe von Knotendeformationen, -beschleunigungen und Schnittgrößen infolge von Zugüberfahrten. Ziel war es, die steifigkeitsbeeinflussende Wirkung der einzelnen Randbedingungen separat zu identifizieren. Zusätzliche Dämpfungsbeiträge lassen sich hingegen nicht ohne weiteres bestimmten baulichen Gegebenheiten zuordnen. Sie führen ohnehin zu einer Erhöhung der globalen Systemdämpfung und lassen sich dementsprechend durch Vergrößerung des globalen Dämpfungsparameters numerisch abbilden. Eine separate Betrachtung der baulichen Randbedingungen wurde im Hinblick auf die Dämpfung daher nicht vorgenommen.

Als Berechnungsprogramm kam die Software InfoCAD ${ }^{\circledR}$ [27] zum Einsatz, mit der sich dynamische Zeitschrittberechnungen nach dem Newmark-Integrationsverfahren durchführen lassen, vgl. Abschnitt 2.4. Bei der Simulation von Brückenüberfahrten werden die Achslasten der Züge vereinfacht als wandernde Einzellasten abgebildet. Auf eine Berücksichtigung der Wagenmassen sowie der Fahrzeug-Fahrweg-Interaktion wird somit verzichtet. Unter Ansatz der in Bild 2.8 dargestellten Zusatzdämpfung liefert dieses Verfahren ausreichend ge- 
naue Ergebnisse (vgl. [16] und [17]). Die für die Simulation verwendeten Züge können aus einer programminternen Bibliothek ausgewählt oder individuell definiert werden. Der Zug bildet dann einen Lastfall, dem eine Fahrstrecke auf dem Tragwerk sowie eine Geschwindigkeit zugewiesen werden (vgl. Bild 5.1). Es wurde vereinfacht von linearen viskosen Dämpfungseigenschaften ausgegangen. Die Dämpfungsmatrix wurde unter Ansatz der Systemdämpfung im Bereich der Grundeigenfrequenz nach dem Rayleigh-Prinzip aufgebaut, vgl. [59]

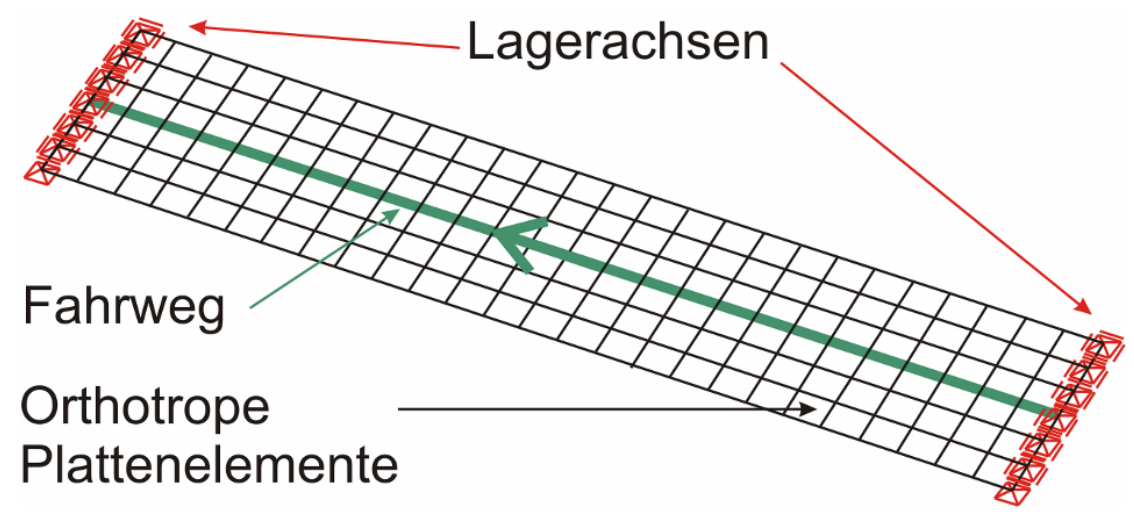

\section{Bild 5.1: Prinzipdarstellung eines numerischen Modells zur Simulation von Zugüberfahrten mit InfoCAD}

Die WIB-Überbauten wurden als liniengelagerte Platten unter Verwendung orthotroper Plattenelemente modelliert. Die äquivalenten Plattendicken ergaben sich dabei aus den zuvor durchgeführten Berechnungen der Verbundquerschnittswerte. Es wurden linear-elastische Materialeigenschaften unter Verwendung eines Vergleichs-E-Moduls definiert. Auf eine Modellierung der Endquerträger, welche die Lasten aus der WIB-Platte in die einzelnen Elastomerlager weiterleiten, wurde vereinfachend verzichtet. Bei eingleisigen Überbauten ist der Einfluss der Orthotropie auf die ersten Biegeeigenfrequenzen und die Beanspruchung infolge von Zugüberfahrten vernachlässigbar gering, da Querbiegungseffekte nur eine untergeordnete Rolle spielen. Bei zweigleisigen Überbauten hingegen sollte darauf geachtet werden, eine deutlich reduzierte Steifigkeit in Plattenquerrichtung anzusetzen, die unter Berücksichtigung eines gerissenen Stahlbetonquerschnittes ermittelt werden sollte. Bei Brücken, die aus zwei durch eine Längsfuge getrennten Überbauten bestehen, wurde zunächst nur eine der beiden Überbauhälften modelliert. In Abschnitt 5.5 wird beschrieben, wie die Kopplung der beiden Überbauten infolge der durchgehenden Schotterschicht numerisch umgesetzt wurde. 


\subsection{Randkappen und Schutzschicht}

Folgende Bauteile lassen sich als Ausrüstungselemente einer Eisenbahnbrücke begrifflich zusammenfassen, vgl. hierzu Bild 5.2.

- Randkappen mit Schotterbegrenzungsbalken

- Darauf befestigte Zusatzelemente wie Kabelkanäle, Geländer, evtl. Schallschutzwände, Fahrleitungen, Signalschilder u.ä.

- Schotterbett und Gleise (siehe nächster Abschnitt)

Außerdem befinden sich zwischen WIB-Platte und Schotterbett eine mechanisch widerstandsfähige Schicht aus Beton von mindestens $50 \mathrm{~mm}$ [8] sowie ein Abdichtungsbelag. Diese Schutzschichten schützen das tragende Bauteil vor mechanischen Einwirkungen von Oberbaumaschinen und vor dem Eindringen von Oberflächenwasser. Eine detaillierte Beschreibung der konstruktiven Durchbildung von Eisenbahnbrücken findet man in [41].

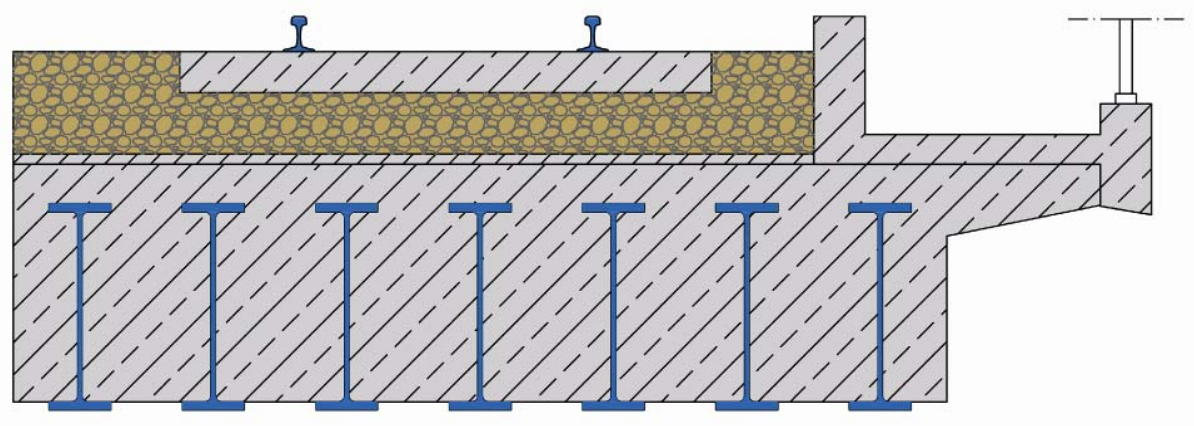

\section{Bild 5.2: WIB-Querschnitt mit Betonschutzschicht und Ausrüstungsele- menten}

Während die Massen der angeführten Ausrüstungselemente und der Betonschutzschicht im Rahmen der dynamischen Bemessung in der Regel berücksichtigt werden, bleiben ihre Beiträge zur Systemsteifigkeit bisher unberücksichtigt. Jedoch gerade die Randkappen und die Schutzbetonschicht können einen signifikanten Steifigkeitsbeitrag leisten. Für die Höhe dieses Steifigkeitsbeitrags ist entscheidend, ob und in welchem Maß Schubkräfte in den Verbindungsfugen zwischen Brückenquerschnitt und Ausbauelementen übertragen werden können. Folgende beiden Extremfälle kommen in Frage:

- Schubweiche Verbindung: Es werden keine Schubkräfte in den Verbindungsfugen übertragen; Lediglich die Eigenträgheitsmomente von Kappe und Schutzschicht gehen additiv in das Gesamtträgheitsmoment des Überbaus ein. 
- Schubstarre Verbindung: Schubkräfte können vollständig in den Verbindungsfugen übertragen werden; die einzelnen Querschnittsteile bilden einen Gesamtquerschnitt mit gemeinsamer Schwerelinie; bei der Ermittlung des Gesamtträgheitsmomentes kommen zu den Eigenträgheitsmomenten die Steineranteile hinzu.

Anhand von zehn Überbauten (siehe Tabelle 5.1) wurde untersucht, wie sich die Steifigkeitsbeiträge von Kappe und Schutzschicht unter der Annahme einer schubweichen bzw. schubstarren Verbindung auf das dynamische Verhalten von WIB-Überbauten auswirken. Dabei wurden die vorhandenen geometrischen Abmessungen der Bauteile berücksichtigt, die in allen Fällen der vorgegebenen Regelausführung entsprachen. In den numerischen Modellen wurde die zusätzliche Steifigkeit durch eine entsprechende Vergrößerung des Vergleichs-EModuls erreicht.

\section{Tabelle 5.1: Einfluss von Kappe und Schutzschicht auf $n_{0}$; Gegenüber- stellung mit Messwerten}

\begin{tabular}{|c|c|c|c|c|c|c|c|c|}
\hline \multirow[b]{2}{*}{ Brücke } & \multirow[b]{2}{*}{ Тур } & \multirow[b]{2}{*}{$\mathrm{L}_{\mathrm{s}}[\mathrm{m}]$} & \multicolumn{4}{|c|}{ Grundeigenfrequenz $\mathrm{n}_{0}[\mathrm{~Hz}]$} & \multirow[b]{2}{*}{ (3) / (1) } & \multirow[b]{2}{*}{ (4) / (3) } \\
\hline & & & $\begin{array}{c}\text { (1) } \\
\text { ohne Aus- } \\
\text { bauelem. }\end{array}$ & $\begin{array}{c}\text { (2) } \\
\text { schubw. } \\
\text { Anschluss }\end{array}$ & $\begin{array}{c}\text { (3) } \\
\text { schubst. } \\
\text { Anschluss }\end{array}$ & $\begin{array}{c}(4) \\
\text { Mess- } \\
\text { wert }\end{array}$ & & \\
\hline Glückaufstraße & $2 * 1 \mathrm{WiB}$ & 12,9 & 4,7 & 5,5 & 8,0 & 9,4 & 1,68 & 1,18 \\
\hline Hertefelder Str. B-HH & $2 * 1 \mathrm{WiB}$ & 13,5 & 5,4 & 6,0 & 8,3 & 9,4 & 1,55 & 1,13 \\
\hline B 477 & $2 * 1 \mathrm{WiB}$ & 15,9 & 4,5 & 4,9 & 6,2 & 6,4 & 1,37 & 1,04 \\
\hline Hackbuschstraße & $2 * 1 \mathrm{WiB}$ & 19,5 & 3,6 & 3,8 & 4,8 & 5,3 & 1,36 & 1,10 \\
\hline Große Erft & $1 \mathrm{WiB}$ & 19,8 & 3,6 & 3,9 & 4,8 & 5,3 & 1,33 & 1,10 \\
\hline Rote-Kreuz-Straße & $1 \mathrm{WiB}$ & 20,1 & 2,9 & 3,7 & 4,5 & 4,7 & 1,56 & 1,04 \\
\hline Erftkanal & $1 \mathrm{WiB}$ & 21,2 & 2,9 & 3,1 & 3,9 & 4,4 & 1,37 & 1,12 \\
\hline Klosterbuschweg & $2 * 1 \mathrm{WiB}$ & 21,2 & 3,4 & 3,6 & 4,5 & 5,3 & 1,33 & 1,17 \\
\hline Goethestraße K-AC & $2 * 1 \mathrm{WiB}$ & 21,9 & 3,7 & 3,9 & 4,7 & 5,0 & 1,27 & 1,06 \\
\hline Erftalstraße K-AC & $2 * 1 \mathrm{WiB}$ & 24,6 & 2,7 & 2,8 & 3,1 & 3,5 & 1,12 & 1,14 \\
\hline
\end{tabular}

Zunächst wurde der Einfluss auf Eigenfrequenzen untersucht. Dabei ist es unerheblich, ob die Grundeigenfrequenzen $n_{0}$ mit InfoCAD oder mit der Handformel nach GI. (2-8) berechnet werden. Beide Methoden liefern annähernd gleiche Ergebnisse.

Die Ergebnisse in Tabelle 5.1 zeigen, dass bei einer Berücksichtigung der Steifigkeitsbeiträge von Kappe und Betonschutzschicht unter Annahme eines schubstarren Verbundes Ergebnisse für $\mathrm{n}_{0}$ erzielt werden, die sich in der Größenordnung der gemessenen Werte befinden, aber dennoch auf der sicheren Seite liegen (siehe letzte Spalte in Tabelle 5.1). 
Weiterhin wird deutlich, dass sich die zusätzlichen Steifigkeitsbeiträge besonders bei Brücken kürzerer Spannweite auswirken (siehe vorletzte Spalte in Tabelle 5.1). Die Abmessungen von Kappe und Schutzschicht sind von der Stützweite weitestgehend unabhängig, d.h. sie verfügen über annähernd gleiche Eigenträgheitswerte und Querschnittsflächen. Bezogen auf den Ausgangsquerschnitt ist der prozentuale Beitrag zur Systemsteifigkeit von Kappe und Schutzschicht bei kurzen Brücken mit geringen Ausgangssteifigkeiten somit deutlich größer als bei weitgespannten Brücken. Dieser Zusammenhang erklärt die deutlich größeren Abweichungen zwischen theoretischen und gemessenen Eigenfrequenzen bei kurzen Brücken im Vergleich zu den Abweichungen bei langen Brücken, vgl. Abschnitt 3.1.2. Die aussteifende Zusatzwirkung von Kappe und Schutzschicht wird mit zunehmender Stützweite immer geringer.

a)

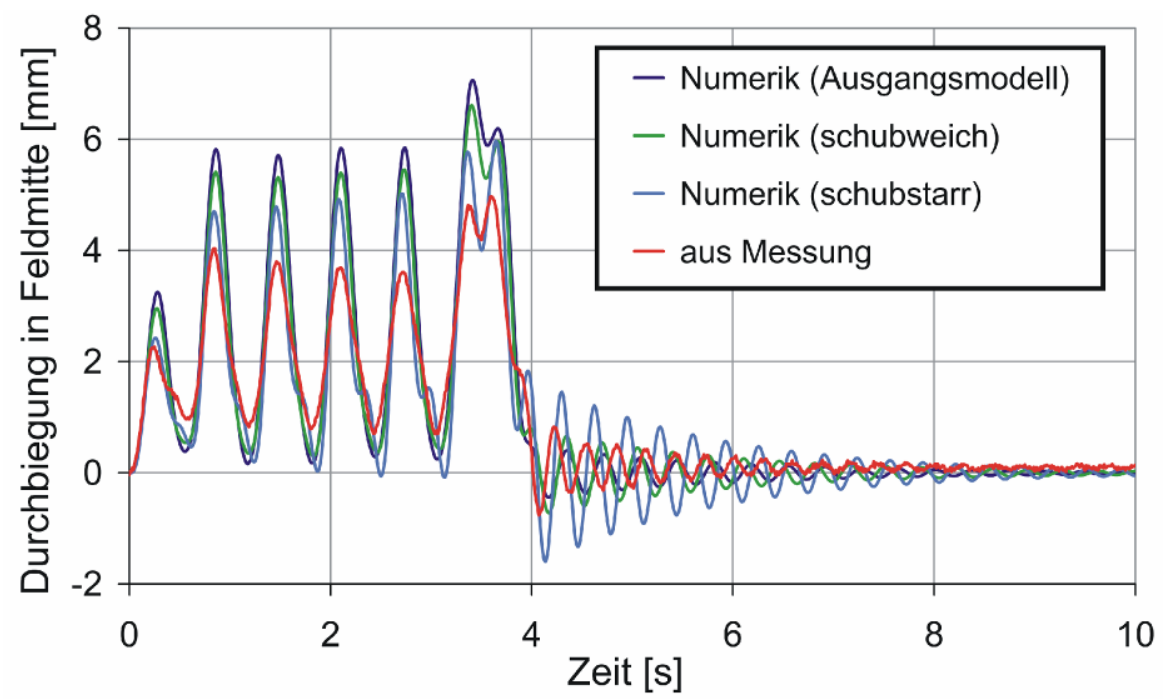

b)

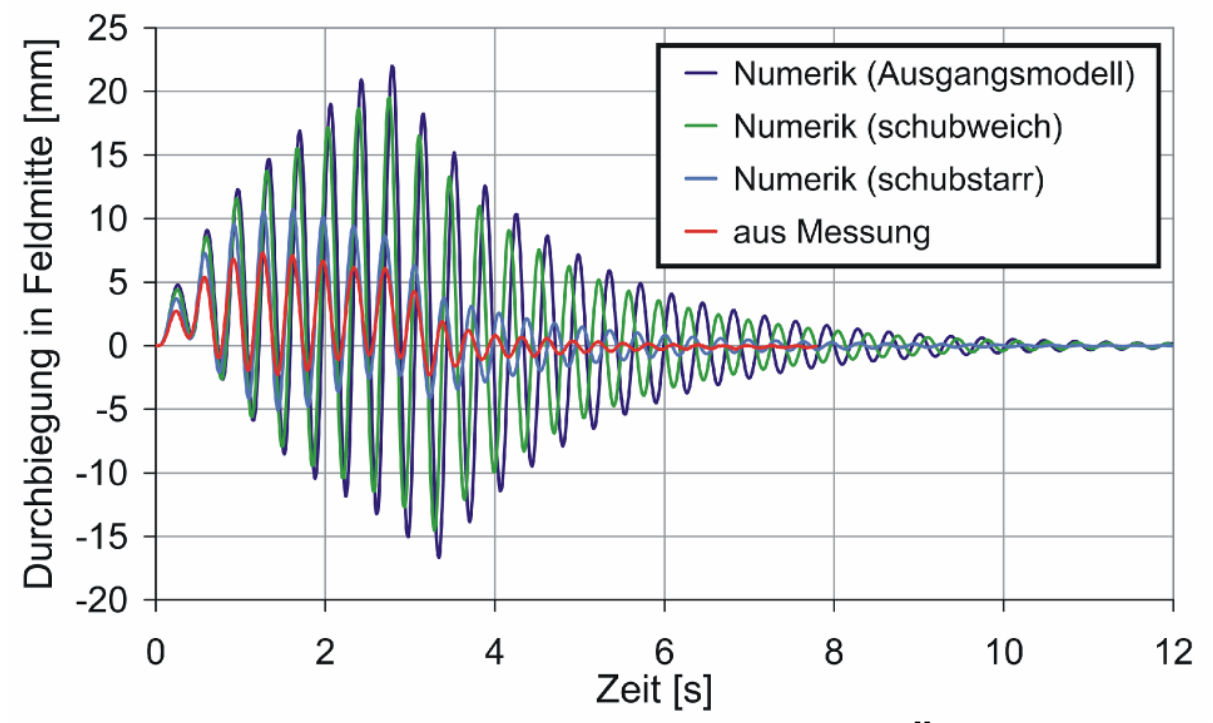

Bild 5.3: Durchbiegungsverläufe infolge von a) RE-Überfahrten (Lok hinten) mit v = $130 \mathrm{~km} / \mathrm{h}$ b) ICE-Überfahrten mit v = $245 \mathrm{~km} / \mathrm{h}$; Vergleich Messung und Numerik 
Neben dem Einfluss der zusätzlichen Steifigkeitsbeiträge auf die Eigenfrequenzen wurden auch deren Auswirkungen auf die dynamische Anregung von WIBTrägern untersucht. Exemplarisch für die durchgeführten Berechnungen sind in Bild 5.3 Verformungszeitverläufe dargestellt, die sich bei Überfahrtsimulationen mit RE- und ICE-Zügen auf der Brücke EÜ Erfttalstraße ergeben. Dabei wurde die gemessene Dämpfung von $3 \%$ in Ansatz gebracht. Den berechneten Verläufen werden aus Messungen abgeleitete Verläufe gegenübergestellt, die im Rahmen eines Brücken-Monitoring bestimmt wurden. Die Messkampagne wird derzeit an der Brücke Erfttalstraße auf der HGV-Strecke Köln-Düren fortgesetzt. Einzelheiten hierzu sind [49] zu entnehmen. Während die am Ausgangsmodell ermittelten Verläufe sehr deutlich von den gemessenen Verläufen abweichen, wird durch die Berücksichtigung zusätzlicher Steifigkeiten eine Annäherung der berechneten an die gemessenen Verläufe erzielt.

Bild 5.4 veranschaulicht, wie sich die Modifikation des Ausgangsmodells durch die schubstarr angeschlossenen Ausbauelemente auf die kritischen Geschwindigkeiten auswirkt. Das maximal zu erwartende Biegemoment wird beim steiferen System erst bei deutlich höheren Geschwindigkeiten erreicht. Eine Erhöhung der Systemdämpfung von 1,6 \% (normativer Wert) auf 3,0 \% (gemessener Wert) führt durch den Abbau von Amplitudenspitzen jeweils zu einer verringerten Überbaubeanspruchung mit gleich bleibenden kritischen Geschwindigkeiten.

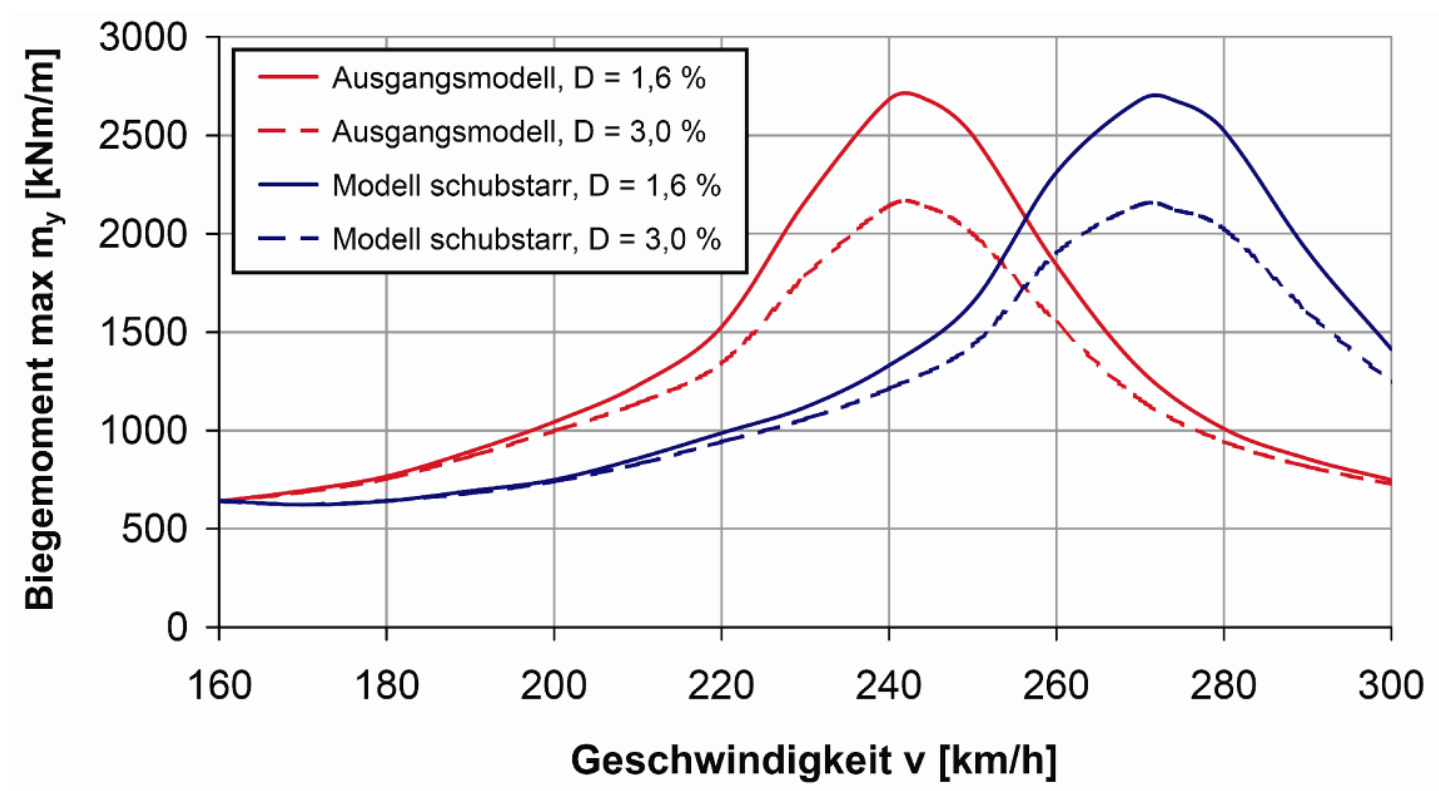

Bild 5.4: Maximale Biegemomente resultierend aus simulierten ICE-3Überfahrten; dynamische Analysen mit unterschiedlichen Dämpfungsparametern und Modellierungsansätzen 


\subsection{Schotterbett und Gleise}

Alle in Tabelle 3.1 aufgelisteten Brücken sind mit einem Schotteroberbau ausgerüstet. Die Oberbauart Feste Fahrbahn wird daher in diesem Beitrag nicht näher untersucht. Diesbezüglich wird auf [57] verwiesen. Um den Steifigkeitsbeitrag von Gleisen und Schotter separat erfassen zu können, wurden Brückenüberbau, Schotter und Schienen getrennt voneinander modelliert. Der Brückenüberbau wurde, wie in Abschnitt 5.1 beschrieben, durch orthotrope Plattenelemente und die Schienen durch Stabelemente gebildet. Die Schotterschicht wurde mit Hilfe von horizontalen und vertikalen Federn abgebildet (schematische Darstellung in Bild 4.12a), die eine Verbindung von Schienen und Brücke herstellen. Die Steifigkeit der vertikalen Bettungsfedern setzt sich zusammen aus den Dehnsteifigkeiten des Schotters und der Zwischenlage. Aus Versuchen abgeleitete Werte können z. B. [28] entnommen werden. Im Rahmen der numerischen Untersuchung wurden die Steifigkeiten der Einzelfedern unter Annahme einer Bettung von $100 \mathrm{MN} / \mathrm{m}^{2}$ berechnet.

Die Steifigkeitseigenschaften der horizontalen Federelemente wurden auf Grundlage des DIN-Fachberichts 101 [11] festgelegt. Dort wird in Anhang K der Längsverschiebewiderstand angegeben, der sich bei einer Relativverschiebung zwischen Gleis und Brückendeck einstellt (siehe Bild 4.12c). Daraus lässt sich ein nichtlineares Federgesetz ableiten, das bei der Definition der Federeigenschaften im numerischen Modell verwendet wurde. Die horizontalen Federelemente repräsentieren somit eine Art Schubsteifigkeit zwischen Schienen und Brücke, wobei die maximal aufnehmbaren Schubkräfte bei einer Relativverschiebung von 2,0 mm erreicht werden. In [30] wurde ein prinzipiell ähnliches Federmodell aus Versuchen abgeleitet, das die nichtlinearen Eigenschaften des Schotterbetts ebenfalls berücksichtigt.

Die Ergebnisse der numerischen Berechnungen (Tabelle 5.2) zeigen, dass der Einfluss der Federelemente auf die Eigenfrequenzen gering ist. Bei allen untersuchten Brücken bewirkt die Implementierung der Federelemente Erhöhungen der Grundeigenfrequenzen kleiner $3 \%$.

Die Ergebnisse der Überfahrtsimulationen (ICE-1-Überfahrten auf der Brücke Erftalstraße), die unter Ansatz der normativ vorgegebenen Dämpfung von $1,6 \%$ durchgeführt wurden, zeigen allerdings deutlich die versteifende Wirkung des Schotteroberbaus, siehe Bild 5.5. Während die vertikalen Federn praktisch wirkungslos in Bezug auf maximal auftretende Biegemomente sind, ergeben sich bei einem System mit "Schubfedern“ zum einen kleinere Momente, zum anderen vergrößert sich die maßgebende kritische Geschwindigkeit merklich. 
Tabelle 5.2: Einfluss von Schotter und Gleisen auf Grundeigenfrequenz und Momentenbeanspruchungen infolge von Zugüberfahrten

\begin{tabular}{|c|c|c|c|c|c|c|c|c|}
\hline \multirow[b]{2}{*}{ Brücke } & \multirow[b]{2}{*}{ Тур } & \multirow{2}{*}{$\mathrm{L}_{\mathrm{s}}[\mathrm{m}]$} & \multicolumn{2}{|c|}{$\mathrm{n}_{0}[\mathrm{~Hz}]$} & \multirow{2}{*}{$\begin{array}{r}\text { Diff. } \\
{[\%]}\end{array}$} & \multicolumn{2}{|c|}{$\mathrm{m}_{\mathrm{ymax}}[\mathrm{kNm}]^{1)}$} & \multirow{2}{*}{$\begin{array}{c}\text { Diff. } \\
{[\%]}\end{array}$} \\
\hline & & & $\begin{array}{c}\text { Ausgangs- } \\
\text { modell }\end{array}$ & $\begin{array}{l}\text { Feder- } \\
\text { modell }\end{array}$ & & $\begin{array}{c}\text { Ausgangs- } \\
\text { modell }\end{array}$ & $\begin{array}{l}\text { Feder- } \\
\text { modell }\end{array}$ & \\
\hline Steinförder Str. & $2 * 1 \mathrm{WiB}$ & 10,4 & 5,91 & 6,16 & 4,2 & 385 & 359 & $-6,8$ \\
\hline В 477 & $2 * 1 \mathrm{WiB}$ & 15,9 & 4,51 & 4,63 & 2,8 & 511 & 460 & $-10,0$ \\
\hline Große Erft & $1 \mathrm{WiB}$ & 19,8 & 3,64 & 3,70 & 1,6 & 334 & 314 & $-6,0$ \\
\hline Erftkanal & $1 \mathrm{WiB}$ & 21,2 & 2,88 & 2,94 & 2,0 & 653 & 563 & $-13,8$ \\
\hline Klosterbuschweg & $2 * 1 \mathrm{WiB}$ & 21,2 & 3,42 & 3,51 & 2,7 & 916 & 801 & $-12,6$ \\
\hline Erftalstr. K-AC & $2 * 1 \mathrm{WiB}$ & 24,6 & 2,74 & 2,79 & 1,8 & 2160 & 2033 & $-5,9$ \\
\hline
\end{tabular}

${ }^{1)}$ Maximale Biegemomente infolge von ICE-1-Überfahrten mit v $=245 \mathrm{~km} / \mathrm{h}$

Vergleicht man die Kurvenverläufe mit denen in Bild 5.4, so zeigt sich eine Übereinstimmung im Hinblick auf die Erhöhung der kritischen Geschwindigkeit. Die versteifende Wirkung des Schotteroberbaus könnte also vereinfacht durch eine Erhöhung der globalen Biegesteifigkeit berücksichtigt werden, wobei die Abminderung der maximal auftretenden Beanspruchungen auf der sicheren Seite liegend vernachlässigt würde.

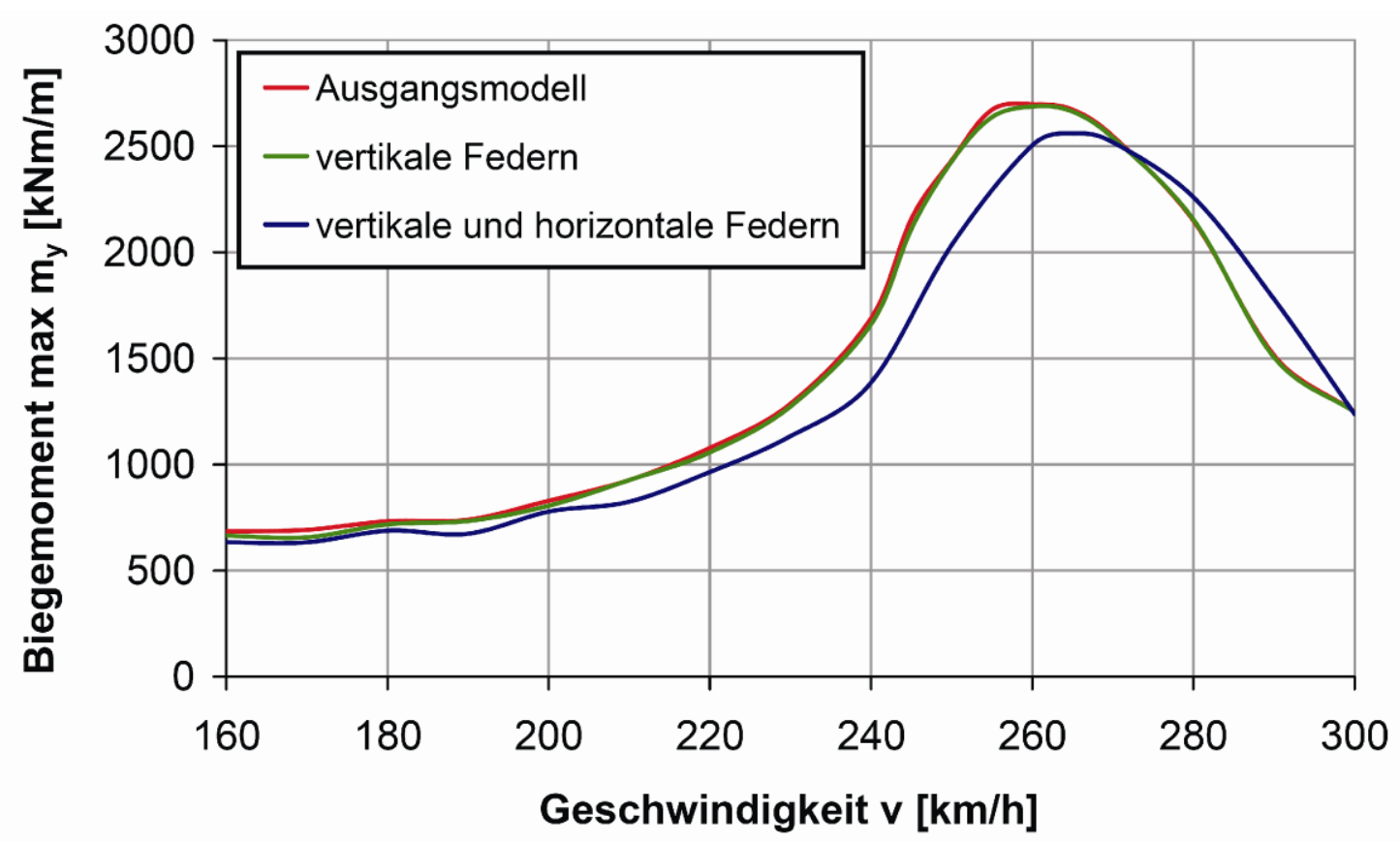

Bild 5.5: Maximale Feldmomente in Abhängigkeit der Zuggeschwindigkeit (ICE-1-Überfahrten) für unterschiedliche numerische Modelle der Brücke Erfttalstraße 


\subsection{Teileinspannung am Auflager}

Die Berechnung von WIB-Brücken in Einfeldträgerbauweise erfolgt in der Regel unter Annahme ideal gelenkiger Auflagerbedingungen. Die realen Lagerbedingungen weichen davon jedoch ab: Da Lagerebene und Systemschwerelinie nicht zusammenfallen, ergeben sich bei Brückendurchbiegungen Verschiebungen in der Lagerebene, die aufgrund von Reibungskräften zu Verzerrungen der Elastomerlager führen. Außerdem werden die Elastomerlager um den entsprechenden Auflagerdrehwinkel verdreht, vgl. Bild 5.6 a und b. Dadurch werden in den Lagern sowohl Rückstellkräfte als auch -momente mobilisiert, die unter Berücksichtigung des als Hebelarm wirkenden Versatzes zwischen Lagerebene und Schwerelinie gemeinsam ein Gesamtrückstellmoment bilden. Durch dieses Rückstellmoment wird eine Teileinspannung des Brückenüberbaus erzeugt, die sich versteifend auf das System auswirkt.

a)

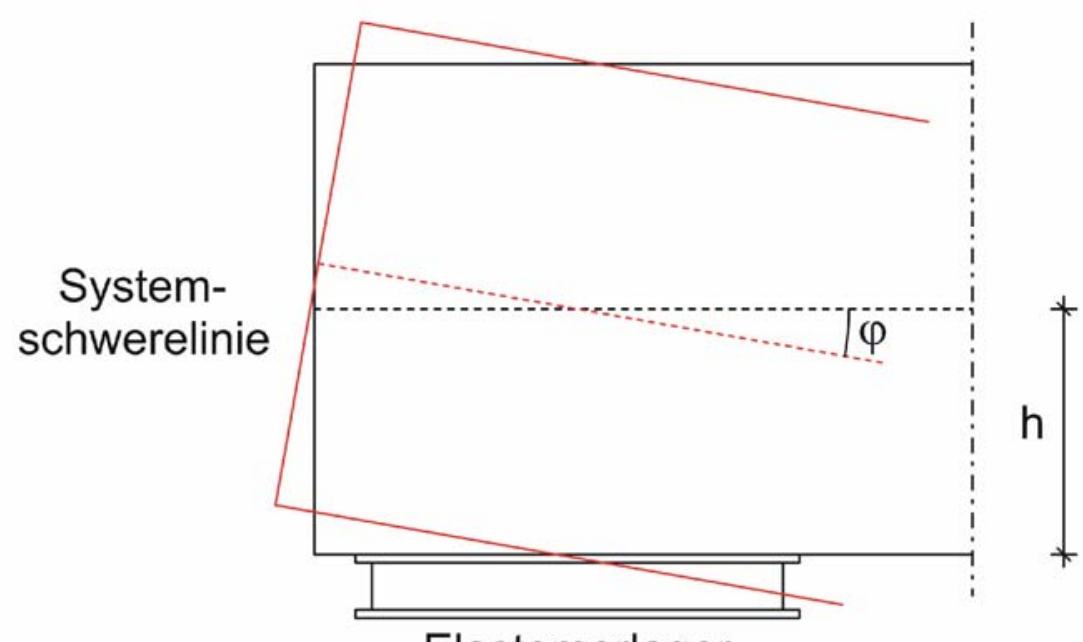

Elastomerlager

b)

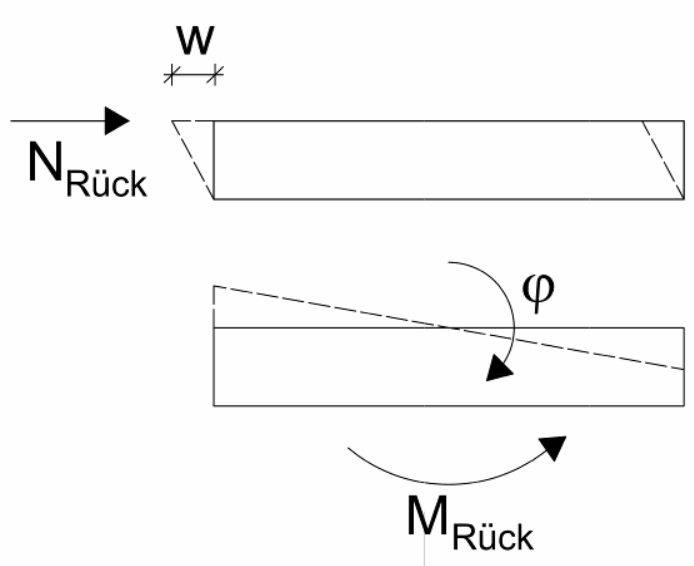

Bild 5.6: a) Verformungsfigur am Auflager sowie b) daraus resultierende Rückstellkräfte und -momente 
Im Rahmen der numerischen Untersuchungen wurde die Teileinspannung am Auflager unter Verwendung von linear-elastischen Drehfedern abgebildet. Zur Ermittlung der jeweils zu verwendenden Drehfedersteifigkeiten, die vom Lagertyp sowie von der Lager- und Systemgeometrie abhängig sind, wurde folgendermaßen vorgegangen: Zunächst wurde geprüft, für welche in Tabelle 3.1 aufgelisteten Brücken hinreichend genaue Informationen zu den ausgeführten Lagern vorlagen. Dies war bei insgesamt zwölf Überbauten der Fall. Nach Vorgabe einer beliebigen Auflagerverdrehung $\varphi$ in den Lagerachsen der ausgewählten Brücken wurden die daraus resultierenden Rückstellreaktionen ermittelt. Die im Elastomerlager hervorgerufenen Rückstellkräfte und -momente können auf Grundlage der DIN EN 1337-3 [9] nach GIn. (5-1) und (5-2) in Abhängigkeit von w und $\varphi$ berechnet werden. Dabei werden neben Angaben zu Auflagergeometrie und -aufbau außerdem der Schubmodul $G$ und ein Faktor $K_{S}$ zur Berechnung des Rückstellmoments benötigt.

$$
\begin{aligned}
& N_{\text {Rück }}=\frac{A \cdot G \cdot w}{T_{e}} \\
& M_{\text {Rück }}=\frac{G \cdot \varphi \cdot a^{\prime 5} \cdot b^{\prime}}{n \cdot t_{i}^{3} \cdot K_{S}}
\end{aligned}
$$

Für kleine Drehwinkel $\varphi$ gilt dabei:

$$
w=\tan \varphi \cdot h
$$

Zur Bestimmung der Drehfedersteifigkeit wurde anschließend der Quotient aus Gesamtrückstellmoment und zugehörigem Drehwinkel gebildet:

$$
c_{\varphi}=\frac{M(\varphi)}{\varphi}=\frac{N_{\text {Rück }}(\varphi) \cdot h+M_{\text {Rück }}(\varphi)}{\varphi}
$$

Die Auswirkungen auf die Grundeigenfrequenzen sind Tabelle 5.3 zu entnehmen. Außerdem sind die Momentenbeanspruchungen angegeben, die sich bei ICE-1-Überfahrten mit $v=245 \mathrm{~km} / \mathrm{h}$ mit und ohne Berücksichtigung der Teileinspannung ergeben. Es zeigt sich, dass die Teileinspannungen am Auflager geringfügige Veränderungen der Eigenfrequenzen hervorrufen, die maximal nur $1,2 \%$ betragen und somit vernachlässigbar sind. Auch die Auswirkungen auf maximal auftretende Biegemomente sind verhältnismäßig klein. Nur in zwei Fällen beträgt die aus der Teileinspannung resultierende Abminderung mehr als $5 \%$. 
Tabelle 5.3: Grundeigenfrequenzen und Momentenbeanspruchungen infolge von Zugüberfahrten mit und ohne Berücksichtigung der Teileinspannwirkung

\begin{tabular}{|c|c|c|c|c|c|c|c|c|}
\hline \multirow{2}{*}{ Brücke } & \multirow{2}{*}{ Typ } & \multirow{2}{*}{$\mathrm{L}_{\mathrm{s}}[\mathrm{m}]$} & \multicolumn{2}{|c|}{$\mathrm{n}_{0}[\mathrm{~Hz}]$} & \multirow{2}{*}{$\begin{array}{r}\text { Diff. } \\
{[\%]}\end{array}$} & \multicolumn{2}{|c|}{$\mathrm{m}_{\mathrm{ymax}}[\mathrm{kNm}]^{1)}$} & \multirow{2}{*}{$\begin{array}{r}\text { Diff. } \\
{[\%]}\end{array}$} \\
\hline & & & ohne $c_{\varphi}$ & mit $\mathrm{c}_{\varphi}$ & & ohne $c_{\varphi}$ & mit $\mathrm{c}_{\varphi}$ & \\
\hline Steinförder Str. & $2 * 1 \mathrm{WiB}$ & 10,4 & 5,91 & 5,93 & 0,4 & 385 & 384 & $-0,3$ \\
\hline B 477 & $2 * 1 \mathrm{WiB}$ & 15,9 & 4,51 & 4,51 & 0,2 & 511 & 510 & $-0,2$ \\
\hline K72 bei Neuhof & $2 * 1 \mathrm{WiB}$ & 16,5 & 4,22 & 4,23 & 0,2 & 496 & 495 & $-0,2$ \\
\hline Goethestr. AC-K & $2 * 1 \mathrm{WiB}$ & 19,4 & 4,36 & 4,38 & 0,5 & 527 & 522 & $-0,9$ \\
\hline Große Erft & $1 \mathrm{WiB}$ & 19,8 & 3,64 & 3,66 & 0,6 & 334 & 326 & $-2,4$ \\
\hline Rote-Kreuz-Str. & $1 \mathrm{WiB}$ & 20,1 & 2,90 & 2,93 & 1,2 & 619 & 577 & $-6,8$ \\
\hline Erftkanal & $1 \mathrm{WiB}$ & 21,2 & 2,88 & 2,92 & 1,1 & 653 & 625 & $-4,3$ \\
\hline Klosterbuschweg & $2 * 1 \mathrm{WiB}$ & 21,2 & 3,42 & 3,43 & 0,3 & 916 & 906 & $-1,1$ \\
\hline Goethestr. K-AC & $2 * 1 \mathrm{WiB}$ & 21,9 & 3,73 & 3,75 & 0,4 & 780 & 789 & 1,2 \\
\hline Havel & $2 * 1 \mathrm{WiB}$ & 22,1 & 3,69 & 3,71 & 0,5 & 773 & 757 & $-2,1$ \\
\hline Erftalstr. K-AC & $2 * 1 \mathrm{WiB}$ & 24,6 & 2,74 & 2,75 & 0,5 & 2160 & 2033 & $-5,9$ \\
\hline
\end{tabular}

1) Maximale Biegemomente infolge von ICE-1-Überfahrten mit $\mathrm{v}=245 \mathrm{~km} / \mathrm{h}$

Stellt man, wie bereits in den Abschnitten 5.2 und 5.3 vorgeführt, die jeweils maximalen Feldmomente in Abhängigkeit möglicher Zuggeschwindigkeiten dar (vgl. Bild 5.4 und Bild 5.5), so zeigt sich beim modifizierten System wiederum eine Zunahme der kritischen Geschwindigkeiten - wenn auch nur geringfügig bei in etwa gleich bleibenden Maximalwerten.

\subsection{Kopplung von nebeneinander liegenden WIB- Überbauten durch Eisenbahnschotter}

\subsubsection{Vorbemerkungen}

Bei einer separaten Modellierung von WIB-Platten, die durch eine Längsfuge getrennt sind, bleibt eine mögliche gegenseitige Beeinflussung der beiden Überbauten unberücksichtigt. Wie in Abschnitt 4.1 erörtert wurde, ergibt sich aber durch die durchgängige Schotterschicht eine Koppelwirkung zwischen den Überbauten. Messungen an der WIB-Brücke Erfttalstraße haben gezeigt, dass gerade bei Anregungen im Resonanzbereich sowohl das belastete als auch das unbelastete Brückendeck mitschwingen, vgl. Bild 4.2. Da die Schotterschicht die einzige Verbindung der beiden Brückenhälften darstellt, kann der Energieeintrag in das unbelastete System nur über den Schotter stattfinden.

In Abschnitt 4 konnte im Rahmen von experimentellen Untersuchungen an Eisenbahnschotter gezeigt werden, dass sich die Kopplung der Überbauten mit 
Hilfe von Federelementen realisieren lässt, die eine Verbindung der jeweiligen Randknoten in vertikaler Richtung herstellen, vgl. Bild 5.7.

Die Frage nach der anzusetzenden Federsteifigkeit und dem zu berücksichtigenden Federgesetz wurde in Abschnitt 4.5 ausführlich diskutiert. Die Ergebnisse dieser Diskussion werden hier noch einmal kurz zusammengefasst:

- Bei der Implementierung von Koppelfedern im numerischen Modell wird als Federsteifigkeit ein Wert von $4350 \mathrm{kN} / \mathrm{m}^{3}$ (bezogen auf die Kontaktfläche zwischen den angrenzenden Schotterschichten) angesetzt.

- Analog zur normativen Vorgabe des Längsverschiebewiderstandes bei der Weiterleitung von horizontalen Beanspruchungen über das Schotterbett (vgl. Bild 4.12c) wird angenommen, dass sich die maximal übertragbare Kraft ab einer Relativverschiebung von 2,0 mm nicht weiter steigern lässt. Diese Annahme führt zu einem bilinearen Federgesetz zur Beschreibung des Widerstands in der Koppelfuge gemäß Bild 4.13.

Wie groß der Einfluss dieser beiden Systemannahmen auf die Ergebnisse numerischer Überfahrtsimulationen ist, wurde mit Hilfe von Parameterstudien untersucht, die im folgenden Abschnitt behandelt werden. Zum einen wurde dabei geprüft, welchen Einfluss eine Variation der Koppelsteifigkeit auf die Systemantwort bei Zugüberfahrten hat. Zum anderen wurde untersucht, wie sich die Wahl des Federgesetzes (linear oder bilinear) auf die Ergebnisse auswirkt.

Die Parameterstudien werden an der Erfttalbrücke durchgeführt. Sie ist für diese Untersuchungen besonders geeignet, weil an ihr zum einen der Koppeleffekt konkret beobachtet wurde und zum anderen ihre Systemmodellierung in Abschnitt 7.2 detailliert erläutert wird. Somit ist das Vorgehen bei der Modellerstellung für den Leser leicht nachvollziehbar.

Aufbauend auf den Parameterstudien werden im daran anschließenden Abschnitt weiterführende numerische Untersuchungen an von der Deutschen Bahn gemessenen WIB-Überbauten behandelt. Dort geht es um die Frage, wie sich unterschiedliche Randbedingungen wie Spannweite und Überbauten-Versatz bei Berücksichtigung des Koppeleffekts auf Eigenfrequenzen und Systembeanspruchungen während Zugüberfahrten auswirken.

\subsubsection{Voruntersuchungen zum Einfluss von Koppelfedern in nu- merischen Modellen}

Mit den in diesem Abschnitt behandelten Parameterstudien wurde untersucht, wie sich das Federgesetz implementierter Koppelfedern auf die Ergebnisse numerischer Überfahrtsimulationen an WIB-Brückenmodellen auswirkt. Grundlage 
für die Untersuchungen bildeten numerische Modelle der Erfttalbrücke, wobei die folgenden zwei Modelltypen verwendet wurden, siehe hierzu Bild 5.7:

- Modelltyp 1: Modellierung des Einzel-Überbaus, der durch Zugverkehr direkt beansprucht wird (Bild 5.7a)

- Modelltyp 2: Modellierung beider Überbauten, Berücksichtigung des Koppeleffekts durch vertikal wirksame Federelemente in der Koppelfuge (Bild $5.7 \mathrm{~b})$

Die Querschnittssteifigkeiten der Überbauten wurden bei beiden Modelltypen realistisch abgebildet, indem die Biegesteifigkeiten so eingestellt wurden, dass sie den gemessenen Steifigkeiten entsprechen. Nähere Hinweise hierzu sowie weitere detaillierte Hinweise zur Modellierung der o. g. Modelltypen sowie zur Durchführung von Überfahrtsimulationen mit InfoCAD enthält Abschnitt 7.

a)

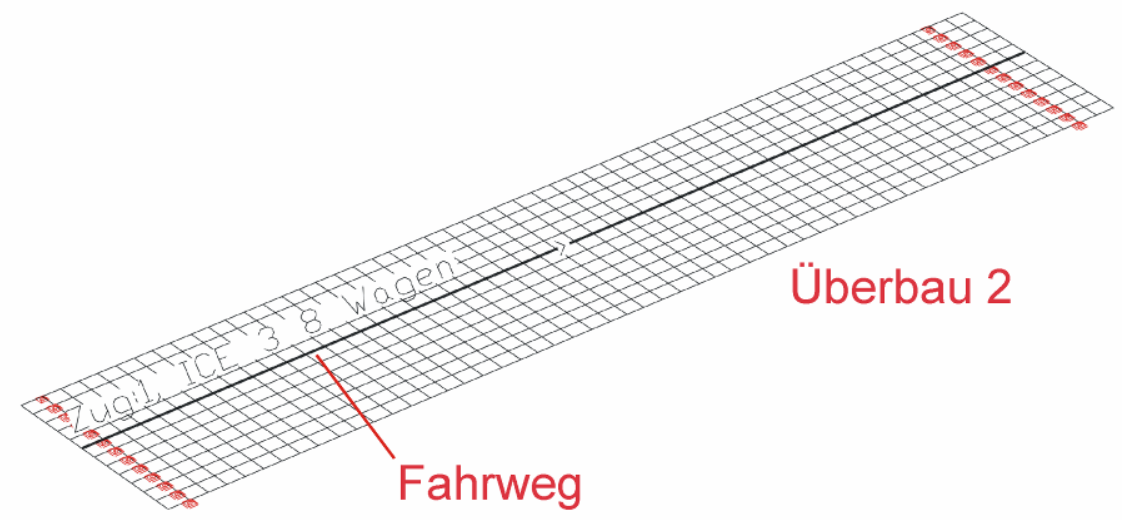

b)

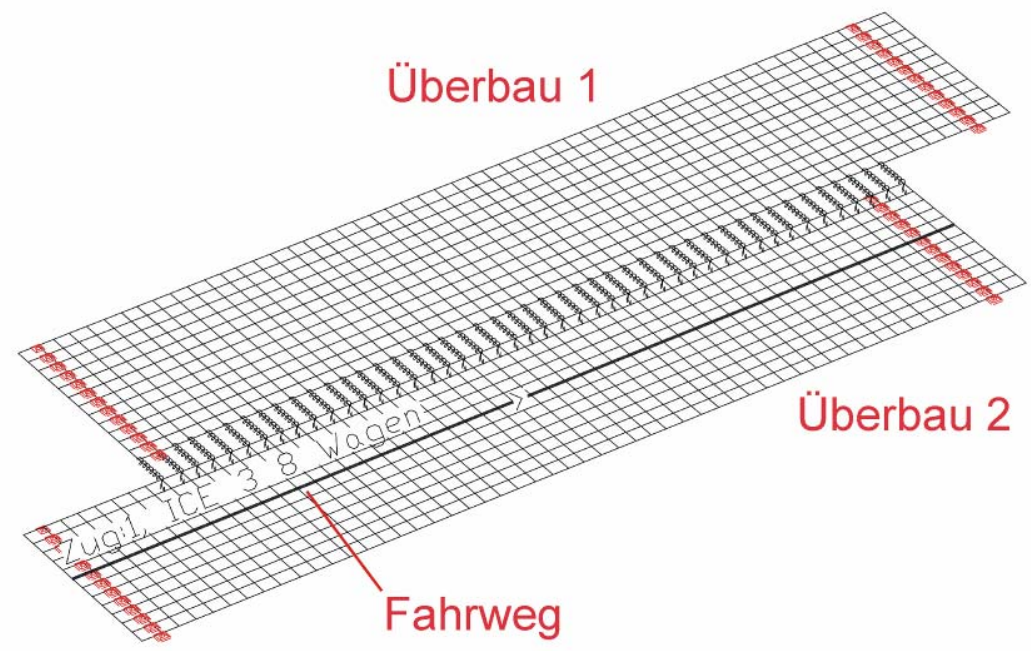

Bild 5.7: Numerische Modelle der Erfttalbrücke für die Durchführung von Parameterstudien zur Untersuchung der Koppelwirkung infolge durchgängiger Schotterschichten a) Modelltyp 1 b) Modelltyp 2 
Die Überfahrtsimulationen wurden unter Verwendung des ICE-3 Zugtyps durchgeführt. Dabei wurde jeweils Überbau 2 durch den Zugverkehr beansprucht, vgl. Fahrwegkennzeichnungen in Bild 5.7.

Zunächst wurde untersucht, welchen Einfluss die absolute Größe der Koppelsteifigkeit auf die Systemantwort bei Zugüberfahrten hat. Dazu wurden Überfahrtsimulationen unter Verwendung von Modelltyp 2 durchgeführt. Den Koppelfedern des Referenzsystems wurde ein bilineares Federgesetz mit einer Anfangssteifigkeit von $4350 \mathrm{kN} / \mathrm{m}^{3}$ und einer Grenzverformung von 2,0 mm zugewiesen, ab der ein "Durchrutschen" einsetzt. (Das Referenzsystem entspricht damit Modell 3 aus Abschnitt 7.2.3.)

Diesem Referenzsystem wurden zwei numerische Modelle gegenübergestellt, bei denen die Anfangssteifigkeit der Koppelfedern um $+20 \%$ erhöht bzw. $-20 \%$ reduziert wurde. Im Vergleich dazu wurde außerdem ein System untersucht, bei dem die Schotterkopplung nicht abgebildet wurde. Hierbei kam Modelltyp 1 zum Einsatz. (Das Vergleichssystem entspricht Modell 2 aus Abschnitt 7.2.3.)

Die Ergebnisse der Untersuchungen an den vier verschiedenen Modellen sind in Bild 5.8 dargestellt. Es zeigt maximal auftretende Beschleunigungen (Bild $5.8 a$ ) bzw. Durchbiegungen (Bild 5.8b) in Abhängigkeit von der Überfahrtgeschwindigkeit. Bis zu einer Geschwindigkeit von etwa $280 \mathrm{~km} / \mathrm{h}$ wirkt sich eine Modifikation des Steifigkeitsparameters um $\pm 20 \%$ nur sehr schwach auf die Simulationsergebnisse aus. Bei höheren Geschwindigkeiten im Bereich der Resonanzanregung nimmt der Einfluss zwar zu, allerdings wird er nicht als signifikant beurteilt, zumal mit der Modifikation der Federsteifigkeiten in diesem Geschwindigkeitsbereich Beschleunigungen und Durchbiegungen erzeugt werden, die im Vergleich zum Referenzmodell eher geringer ausfallen.

Wird die Anfangssteifigkeit der Koppelfedern mit einem Wert von $4350 \mathrm{kN} / \mathrm{m}^{3}$ also eventuell nur ungenau beschrieben, wie in Abschnitt 4.5 in Betracht gezogen, so hat dies offenbar keine signifikanten Auswirkungen auf die numerischen Ergebnisse.

Für den Fall, dass auf die Abbildung des Koppeleffekts mittels Federelementen vollständig verzichtet wird (Modell ohne Koppelfedern in Bild 5.8), vergrößern sich die Bauwerksreaktionen im Bereich kritischer Geschwindigkeiten sowie im Geschwindigkeitsbereich größer 240 km/h allerdings deutlich. 
a)

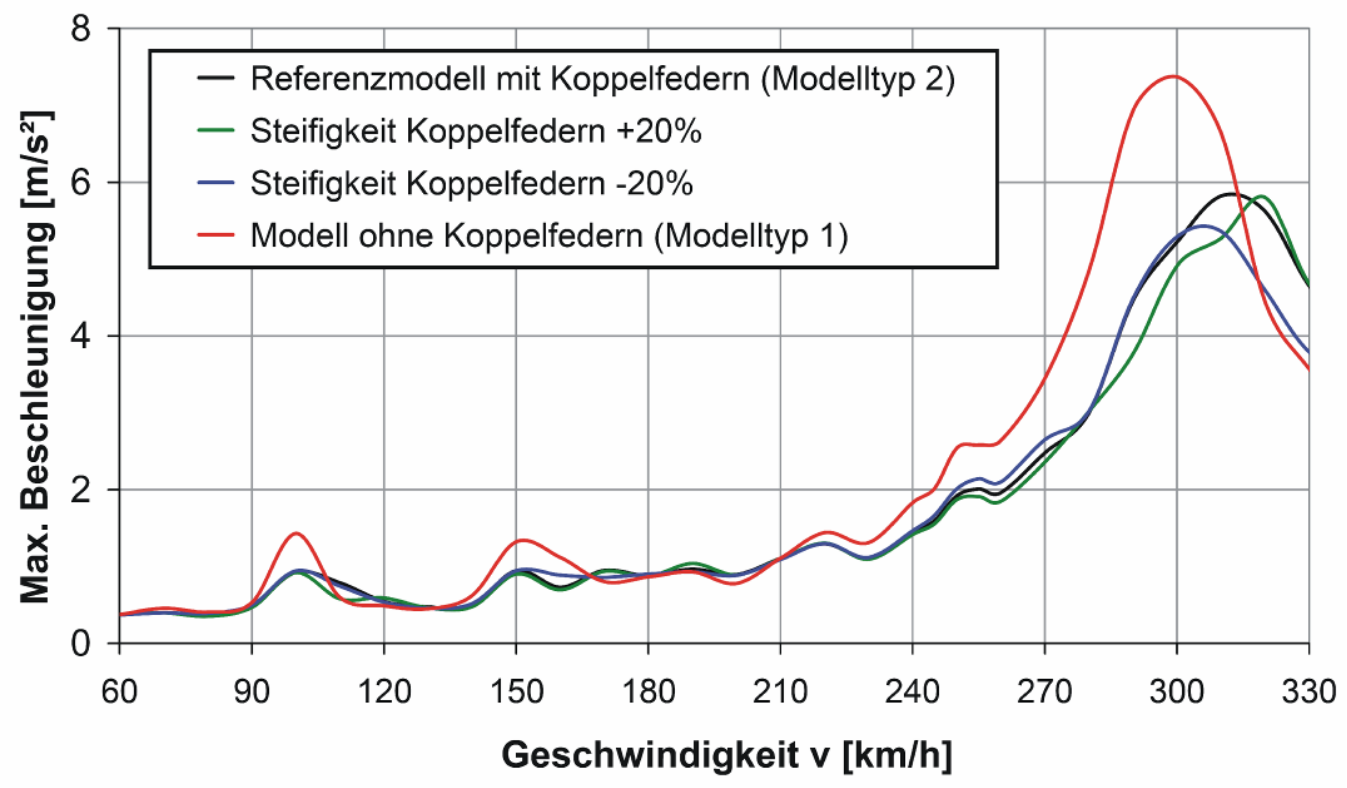

b)

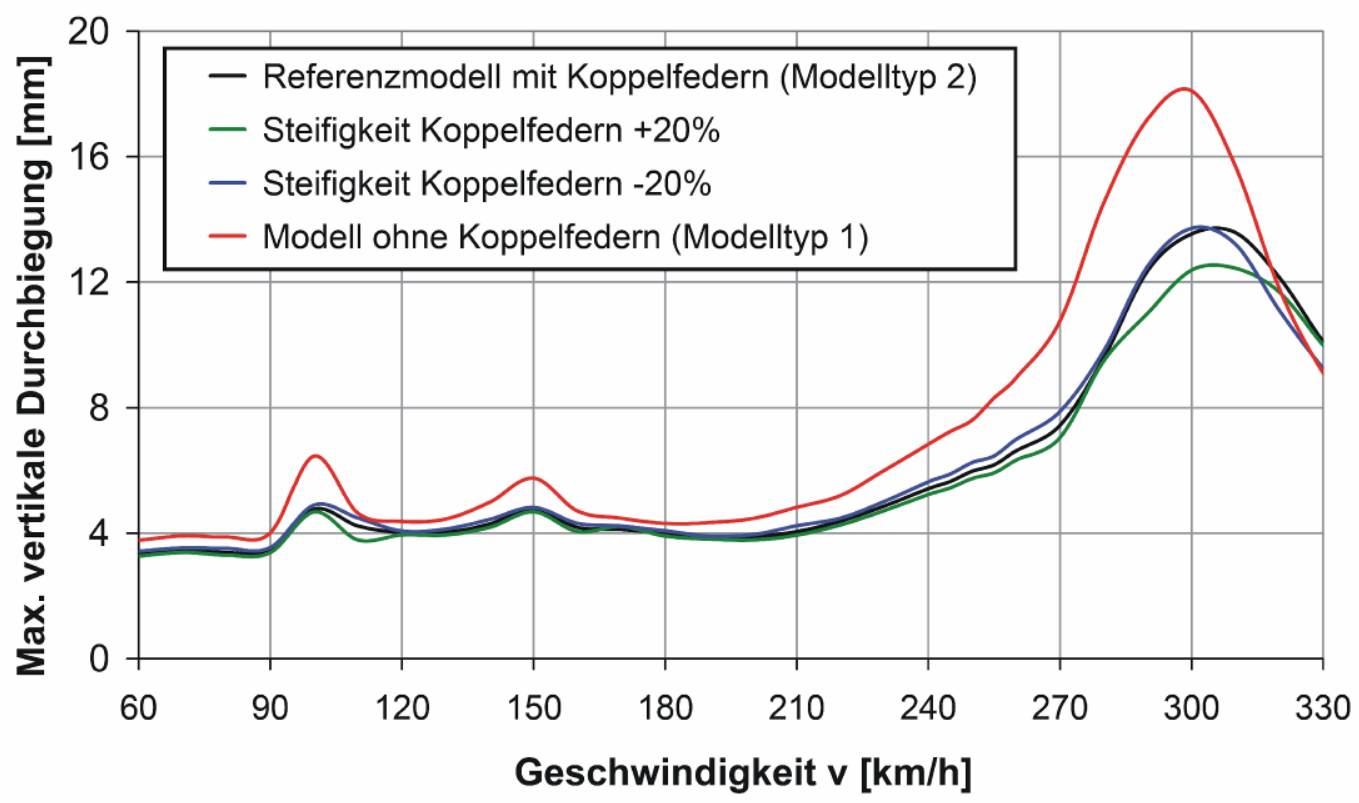

Bild 5.8: Ergebnisse Parameterstudie: Einfluss der Koppelsteifigkeit auf die Systemantwort bei Zugüberfahrten a) Beschleunigungen b) Durchbiegungen

Ausgehend von diesen numerischen Ergebnissen wurde das bereits in Abschnitt 5.3 vorgeschlagene Vorgehen untersucht, die Vergrößerung der Systemsteifigkeit infolge einer baulichen Randbedingung - in diesem Fall infolge des durch den Schotter hervorgerufenen Koppeleffekts - ersatzweise durch eine Erhöhung der Querschnittssteifigkeit unter Verwendung von Modelltyp 1 abzubilden. Zu diesem Zweck wurden weitere Simulationsrechnungen unter Verwendung des eingleisigen Modells ohne Koppelfedern durchgeführt, wobei die Querschnittsbiegesteifigkeit schrittweise erhöht wurde. 
Wie Bild 5.9 zeigt, ergibt sich bei einer Vergrößerung der Steifigkeit um $10 \%$ bis zu einer Geschwindigkeit von etwa $290 \mathrm{~km} / \mathrm{h}$ eine sehr gute Annäherung an die mit dem Referenz-Koppelmodell erzeugten Ergebnisse. Im Falle von höheren Geschwindigkeiten im Resonanzbereich liegen die mit Hilfe des vereinfachten Modells erzeugten Ergebnisse auf der sichern Seite. Sowohl die maximal auftretenden Beschleunigungen (Bild 5.9a) als auch die maximalen Durchbiegungen (Bild 5.9b) sind deutlich größer als die am komplexen Modell unter expliziter Berücksichtigung der Schotterkopplung erzeugten Bauwerksreaktionen.

a)

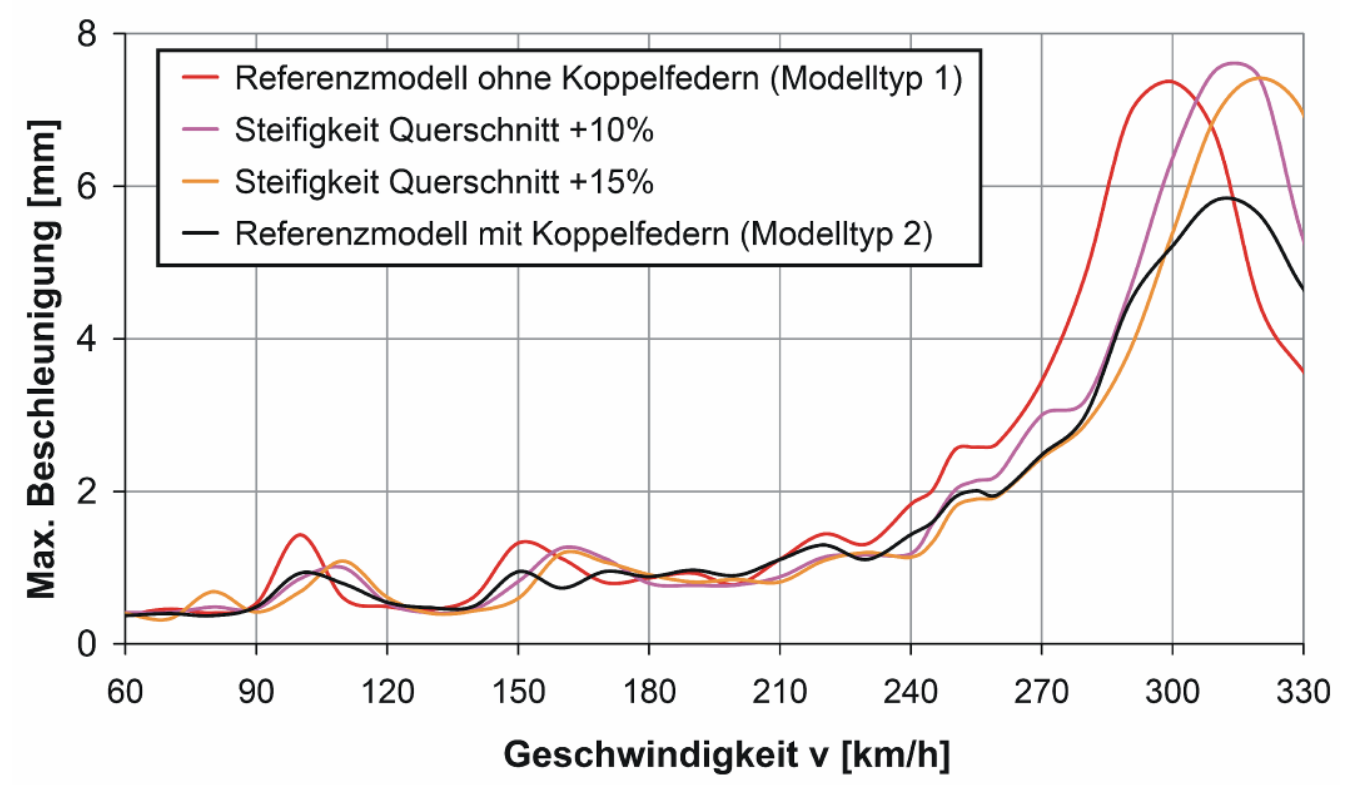

b)

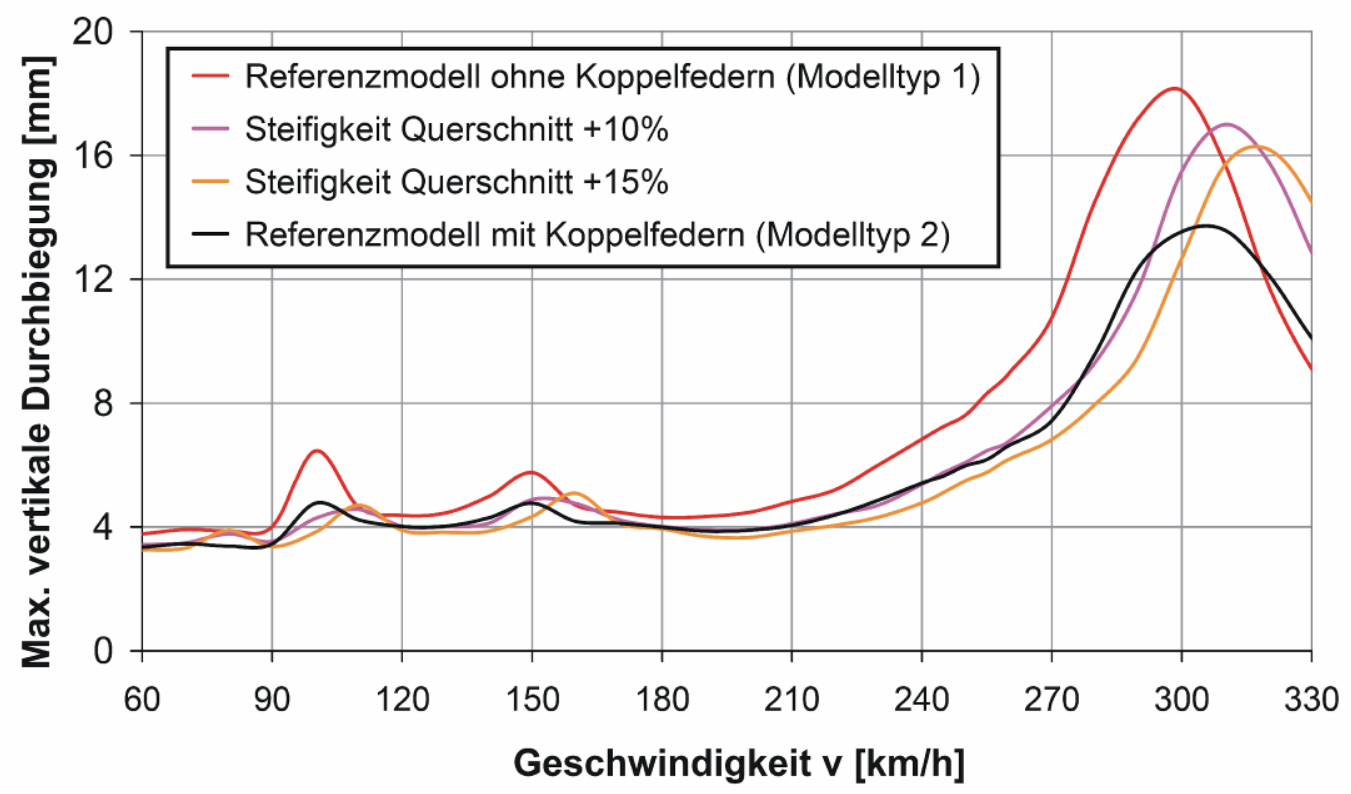

Bild 5.9: Ergebnisse Parameterstudie: Numerische Abbildung des Koppeleffekts durch eine ersatzweise Erhöhung der Querschnittsbiegesteifigkeit bei Verwendung von Modelltyp 1 a) Beschleunigungen b) Durchbiegungen 
Weiterhin wurde untersucht, wie sich das implementierte Federgesetz auf die Simulationsergebnisse auswirkt. Zu diesem Zweck wurde das bereits oben verwendete Referenzsystem mit Koppelfedern einem Modell gegenübergestellt, bei dem linear-elastische Federelemente zur Abbildung des Koppeleffekts eingesetzt wurden. Die Ergebnisse der vergleichenden Berechungen sind in Bild 5.10 dargestellt.

a)

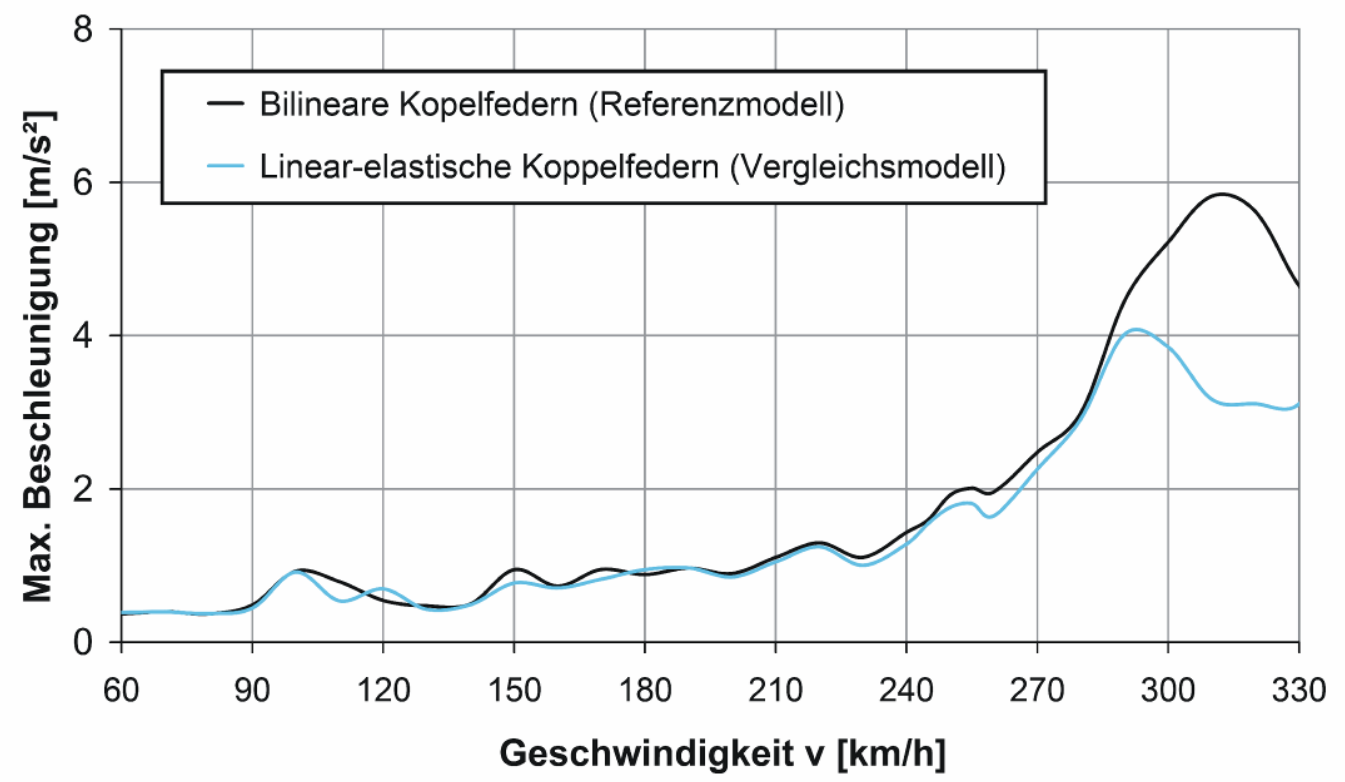

b)

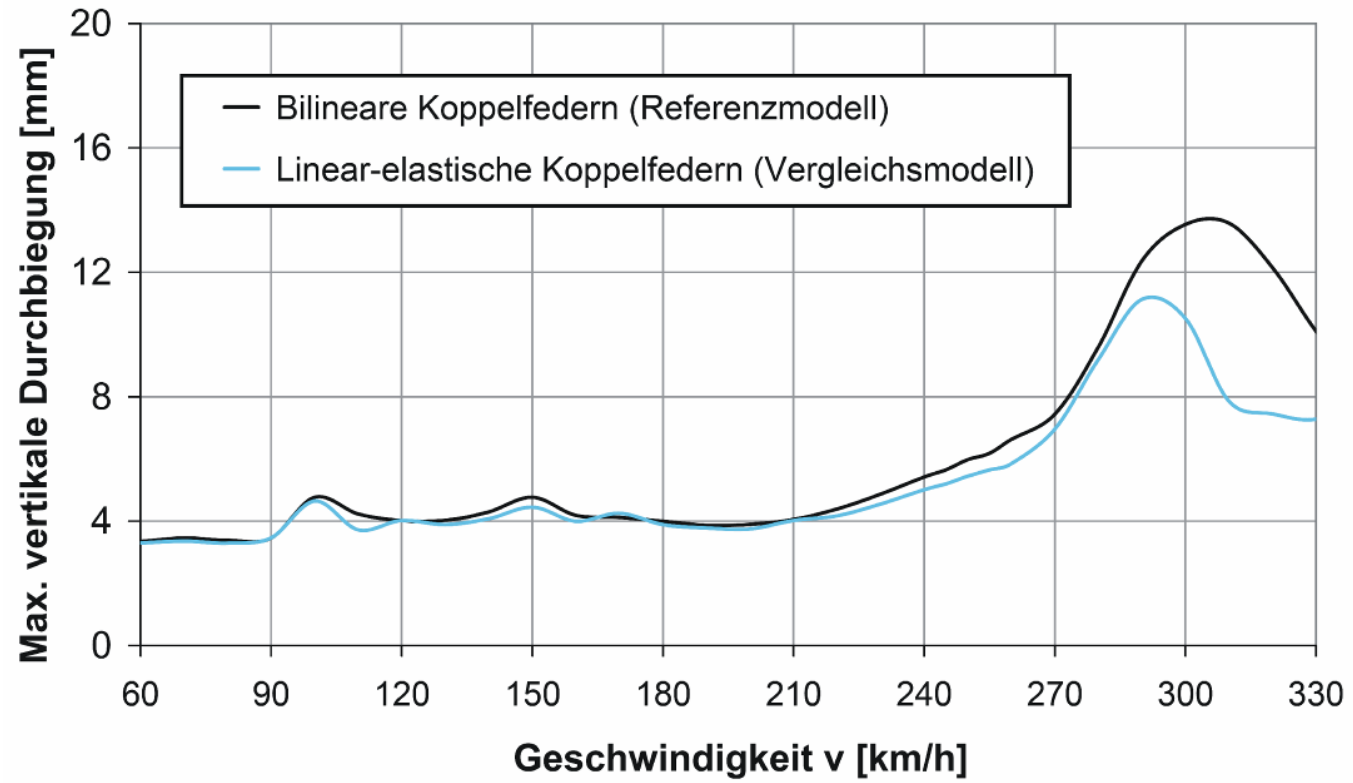

Bild 5.10: Ergebnisse Parameterstudie: Einfluss des Federgesetzes der Koppelfedern auf die Systemantwort bei Zugüberfahrten a) Beschleunigungen b) Durchbiegungen

Im nicht-resonanten Geschwindigkeitsbereich bis etwa $280 \mathrm{~km} / \mathrm{h}$ ist die Art des Federgesetzes weitestgehend unerheblich im Hinblick auf maximal auftretende Beschleunigungen (Bild 5.10a) und Durchbiegungen (Bild 5.10b). Erst bei grö- 
ßeren Geschwindigkeiten im Resonanzbereich ergeben sich voneinander abweichende Verläufe. Die unter Verwendung der bilinearen Federelemente erzeugten Beschleunigungen und Durchbiegungen sind deutlich ungünstiger als die Bauwerksreaktionen des linearen Modells. Die zusätzliche Systemsteifigkeit, die sich aus dem linearen Federgesetz bei Relativverformungen größer 2,0 mm gegenüber dem nichtlinearen Federgesetz ergibt, wirkt sich hier also deutlich aus. Sie führt zu einem verstärkten Energieeintrag in das unbelastete System, aus dem eine abgeminderte Beanspruchung des belasteten Systems resultiert.

Um zu untersuchen, in welchem Maße der bereits diskutierte Durchrutscheffekt bei Verwendung bilinearer Federelemente zum Tragen kommt, wurden in Bild 5.11 die sich maximal einstellenden Relativverformungen in der Koppelfuge in Abhängigkeit von der Überfahrtgeschwindigkeit aufgetragen.

Unter Verweis auf Tabelle 4.4 ist festzustellen, dass die Größenordnung der numerisch ermittelten Relativverformungen sehr gut mit den im Rahmen des Bauwerks-Monitoring gemessenen Werten korrespondiert.

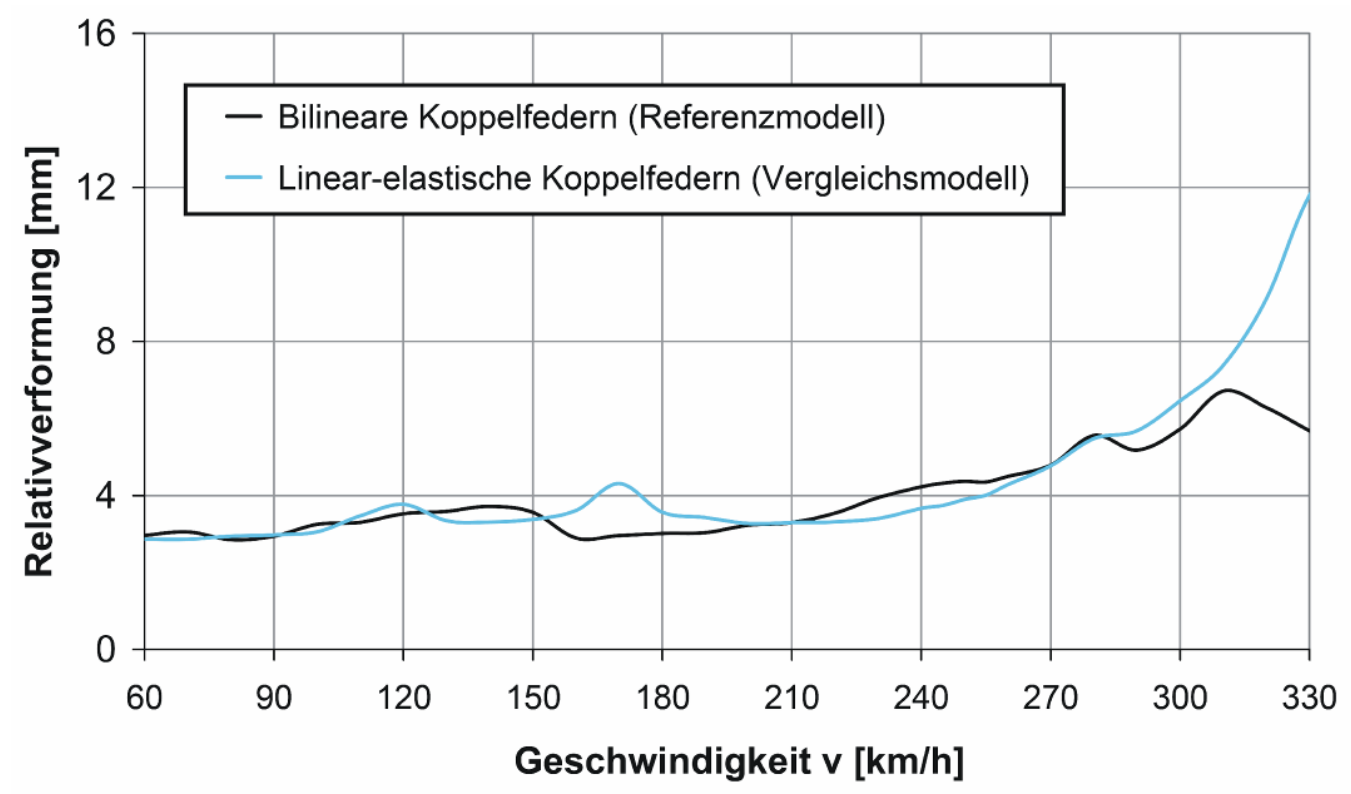

Bild 5.11: Ergebnisse Parameterstudie: Maximale Relativverformungen in der Koppelfuge bei Ansatz eines linearen bzw. bilinearen Federgesetzes der Koppelfedern

Bis zu einer Geschwindigkeit von etwa 290 km/h, die den Übergang zum resonanzanfälligen Bereich darstellt, zeigen sich kaum Unterschiede im Verlauf der beiden Kurven - ein wirksames Durchrutschen in der Koppelfuge findet somit nicht statt. Erst bei höheren Geschwindigkeiten größer $300 \mathrm{~km} / \mathrm{h}$ sind deutliche Unterschiede zu verzeichnen. Hier ist bemerkenswert, dass die Verformungswege der linearen Federelemente erheblich größer sind als die der bilinearen 
Elemente. Dieses Ergebnis deckt sich mit der obigen Feststellung, dass im resonanten Geschwindigkeitsbereich ein verstärkter Lasteintrag über die Koppelfedern in das unbelastete System stattfindet.

Die Ergebnisse der Parameterstudien zeigen, dass das in Abschnitt 4.5 hergeleitete Modell zur Abbildung des Koppeleffekts mittels bilinearer Federelemente zur Verwendung in numerischen Simulationsrechnungen grundsätzlich geeignet ist.

Für den Fall, dass die Anfangssteifigkeit der Koppelfedern mit $4350 \mathrm{kN} / \mathrm{m}^{3}$ nur ungenau abgeschätzt wurde, so sind die Auswirkungen auf die Simulationsergebnisse gering.

Die Frage, ob den Federelementen ein lineares oder ein bilinearen Federgesetz zugewiesen wird, ist nur im resonanzanfälligen Geschwindigkeitsbereich von Bedeutung. Hier ergeben sich bei Verwendung bilinearer Federn deutlich ungünstigere Ergebnisse. Es wird daher trotz des größeren Rechenaufwandes empfohlen, diesen Elementtyp zu verwenden, um die Gefahr zu günstiger Simulationsergebnisse zu vermeiden.

Ein Vergleich der numerisch ermittelten Relativverschiebungen in der Koppelfuge mit gemessenen Werten zeigt eine gute Übereinstimmung und belegt die wirklichkeitsnahe Abbildung des Koppeleffekts mit Hilfe des gewählten Modells.

Es konnte weiterhin gezeigt werden, dass der Koppeleffekt vereinfacht durch eine Erhöhung der Querschnittsbiegesteifigkeit abgebildet werden kann. Dabei werden im Resonanzbereich sowohl im Hinblick auf Beschleunigungen als auch im Hinblick auf Durchbiegungen auf der sicheren Seite liegende Ergebnisse erzeugt.

\subsubsection{Untersuchung der gemessenen Überbauten}

Je nach Kreuzungswinkel zwischen Brückenlängsachse und überquertem Fahrweg sind Brücken mit in Längsrichtung versetzten Überbauten von Brücken ohne Versatz zu unterscheiden. In Bild 5.12 sind beispielsweise numerische Modelle der Brücken Erfttalstraße und Hertefelderstraße dargestellt, bei der die Überbauten um vier bzw. zwei Meter versetzt sind.

Anhand von vier WIB-Brücken wurde untersucht, wie sich die Kopplung auf Eigenfrequenzen und dynamische Anregung auswirken. Dabei wurde, wie in den vorangegangenen Abschnitten ausgeführt, ein bilineares Federgesetz mit einer Anfangssteifigkeit von $4350 \mathrm{kN} / \mathrm{m}^{3}$ in Ansatz gebracht. Analog zu den Abschnitten 5.2 bis 5.4 wurden die numerischen Berechnungen zunächst am Ausgangsmodell unter Berücksichtigung der rechnerischen Steifigkeit des nackten 
Brückenquerschnitts durchgeführt. Anschließend wurde das jeweilige Ausgangsmodell durch Implementierung von Federn zur Abbildung des Koppeleffekts modifiziert, und die numerischen Berechnungen wurden erneut durchgeführt.

a)

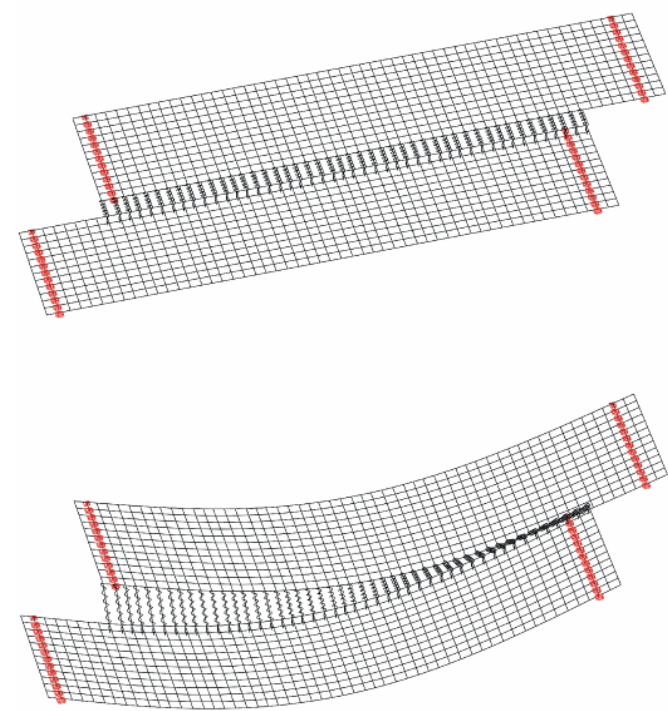

b)
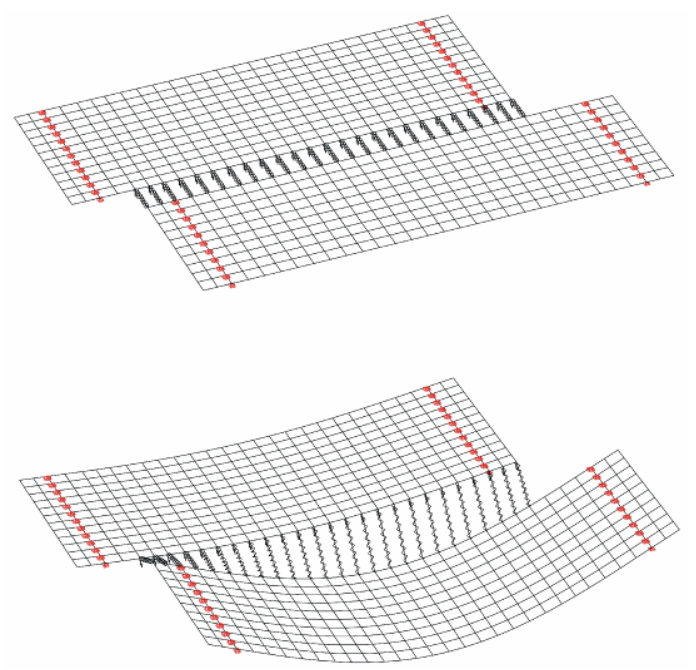

Bild 5.12: InfoCAD-Modelle mit erster Biegeeigenform - Kopplung der beiden versetzt angeordneten eingleisigen Überbauten durch Federn a) EÜ Erfttalstraße b) EÜ Hertefelderstraße

Eine zusammenfassende Gegenüberstellung der Ergebnisse ist Tabelle $5.4 \mathrm{zu}$ entnehmen, wobei erst jeweils die Eigenfrequenzen, maximale Beschleunigungen und Durchbiegungen für die Einzelüberbauten (Ü1 und Ü2), dann für das gekoppelte System („coup“) angegeben sind. Bei Überbauten ohne oder mit kleinem Versatz hat die Kopplung keinen Einfluss auf die Grundeigenfrequenz, vorausgesetzt die Steifigkeiten der beiden Überbauhälften sind annähernd gleich groß. In diesem Fall schwingen beide Überbauhälften in der ersten Eigenform gemeinsam, so dass keine nennenswerten Relativverschiebungen in der Koppelfuge auftreten. Diese Form der Systemantwort würde allerdings voraussetzen, dass beide Brücken gleichzeitig angeregt werden, was bei Zugüberfahrten nicht der Fall ist.

Bei Brücken mit Versatz und Überstand kann der Einfluss der Kopplung auf die erste Eigenfrequenz zunehmen, insbesondere wenn sich die Steifigkeiten der beiden Überbauhälften voneinander unterscheiden. Im Fall der Hertefelderstraße (unterschiedliche Steifigkeiten der Überbauhälften aufgrund unterschiedlicher Plattenbreiten) ergeben sich für die erste Biegeeigenform signifikante Relativverschiebungen in der Koppelfuge, so dass die Koppelfedern wirksam werden (vgl. Bild 5.12b). Im Fall der Erfttalstraße (annähernd identische Steifigkeiten 
der Überbauhälften) sind die Auswirkungen der Koppelfedern auf die Grundeigenfrequenz hingegen gering.

Tabelle 5.4: Auswirkungen der Kopplung auf Eigenfrequenzen und Maximalwerte von Beschleunigungen und Durchbiegungen in Feldmitte (ICE-1-Überfahrt mit v $=245 \mathrm{~km} / \mathrm{h}$ )

\begin{tabular}{|c|c|c|c|c|c|c|}
\hline Brücke & $\begin{array}{c}\mathrm{L}_{\mathrm{s}} \\
{[\mathrm{m}]}\end{array}$ & $\begin{array}{c}\text { Versatz } \\
{[\mathrm{m}]} \\
\end{array}$ & $\begin{array}{c}\text { Überstand } \\
{[\mathrm{m}]^{1)}}\end{array}$ & $\begin{array}{c}\mathrm{n}_{0, \mathrm{cal}, \mathrm{U} 1} \\
{[\mathrm{~Hz}]}\end{array}$ & $\begin{array}{c}\mathrm{n}_{0, \mathrm{cal}, \mathrm{U} 2} \\
{[\mathrm{~Hz}]}\end{array}$ & $\begin{array}{c}\mathrm{n}_{0, \text { cal }, \text { coup }} \\
{[\mathrm{Hz}]}\end{array}$ \\
\hline Glückaufstraße & 12,9 & 0 & 0,6 & 4,73 & 4,73 & 4,74 \\
\hline Klosterbuschweg & 21,2 & 1,0 & 1,0 & 3,42 & 3,42 & 3,42 \\
\hline Hertefelderstraße & 13,5 & 2,0 & 1,0 & 5,38 & 5,11 & 5,59 \\
\hline Erfttalstraße & 24,6 & 4,0 & 0,5 & 2,76 & 2,74 & 2,78 \\
\hline Brücke & $\begin{array}{c}\mathrm{a}_{\max , \mathrm{cal}, \mathrm{U} 1} \\
{\left[\mathrm{~m} / \mathrm{s}^{2}\right]^{2)}}\end{array}$ & $\begin{array}{c}\mathrm{a}_{\max , \mathrm{cal}, \mathrm{U} 2} \\
{\left[\mathrm{~m} / \mathrm{s}^{2}\right]}\end{array}$ & $\begin{array}{c}\mathrm{a}_{\text {max }, \text { cal, coup }} \\
{\left[\mathrm{m} / \mathrm{s}^{2}\right]}\end{array}$ & $\begin{array}{c}\mathrm{u}_{\max , \mathrm{cal}, \mathrm{U} 1} \\
{[\mathrm{~mm}]^{2)}}\end{array}$ & $\begin{array}{c}\mathrm{u}_{\max , \mathrm{cal}, \mathrm{U} 2} \\
{[\mathrm{~mm}]}\end{array}$ & $\begin{array}{c}\mathrm{u}_{\max , \text { cal }, \text { coup }} \\
{[\mathrm{mm}]}\end{array}$ \\
\hline Glückaufstraße & 3,3 & 3,3 & 3,9 & 6,9 & 6,9 & 6,3 \\
\hline Klosterbuschweg & 2,1 & 2,1 & 1,7 & 8,1 & 8,1 & 6,0 \\
\hline Hertefelderstraße & 4,0 & 3,0 & 3,5 & 5,2 & 5,5 & 4,7 \\
\hline Erfttalstraße & 4,9 & 4,7 & 2,9 & 21,9 & 21,0 & 14,0 \\
\hline
\end{tabular}

1) Auskragung der WIB-Platte über die Auflagerachse hinaus

${ }^{2)}$ Beschleunigungen und Verformungen infolge von ICE-1-Überfahrten mit v $=245 \mathrm{~km} / \mathrm{h}$

Erheblich größer ist der Einfluss der Kopplung auf die Ergebnisse von Überfahrtsimulationen. Durch den Energieeintrag in das unbelastete System verringert sich die Beanspruchung des belasteten Überbaus signifikant. Dieser Effekt verstärkt sich mit zunehmendem Versatz, da im Bereich des Versatzes eine Kopplung mit dem Untergrund stattfindet, die sich versteifender auf das System auswirkt als eine Kopplung mit dem verformbaren Nachbarüberbau. So ist Tabelle 5.4 zu entnehmen, dass sich im Fall der Erfttalstraße bei einem Versatz von 4,0 m die maximalen Beschleunigungen infolge einer ICE-1-Überfahrt mit $v=245 \mathrm{~km} / \mathrm{h}$ von etwa $4,8 \mathrm{~m} / \mathrm{s}^{2}$ auf $2,9 \mathrm{~m} / \mathrm{s}^{2}$ reduzieren. Der zulässige Grenzwert von $3,5 \mathrm{~m} / \mathrm{s}^{2}$ nach [8] könnte also bei Verwendung des modifizierten Modells eingehalten werden. Auch die Verformungen in Feldmitte reduzieren sich erheblich - von etwa 21,5 mm (ursprüngliches System) auf $14 \mathrm{~mm}$ (modifiziertes System). Im Vergleich dazu wurden bei ICE-Überfahrten auf der EÜ Erfttalstraße maximale Durchbiegungen von 6,0 bis $8,0 \mathrm{~mm}$ (vgl. Bild 5.14) und maximale Beschleunigungen von 1,8 bis $2,0 \mathrm{~m} / \mathrm{s}^{2}$ (Frequenzanteile bis $30 \mathrm{~Hz}$ berücksichtigt) in Feldmitte gemessen. Das Vorgehen zur Modellierung der Schotterkopplung ist in Abschnitt 7 detailliert beschrieben.

In Bild 5.13 ist zu erkennen, dass sich durch eine Modellierung der Kopplung die kritische Geschwindigkeit leicht erhöht, während sich das maximal zu erwar- 
tende Biegemoment deutlich reduziert. Ein Vergleich mit Bild 5.4 zeigt auch hier, dass sich die Auswirkungen der Kopplung vereinfacht durch eine Erhöhung der globalen Biegesteifigkeit berücksichtigen lassen. Die Reduzierung des Biegemoments würde in diesem Fall zwar nicht abgebildet, es ergäben sich aber auf der sicheren Seite liegende Ergebnisse.

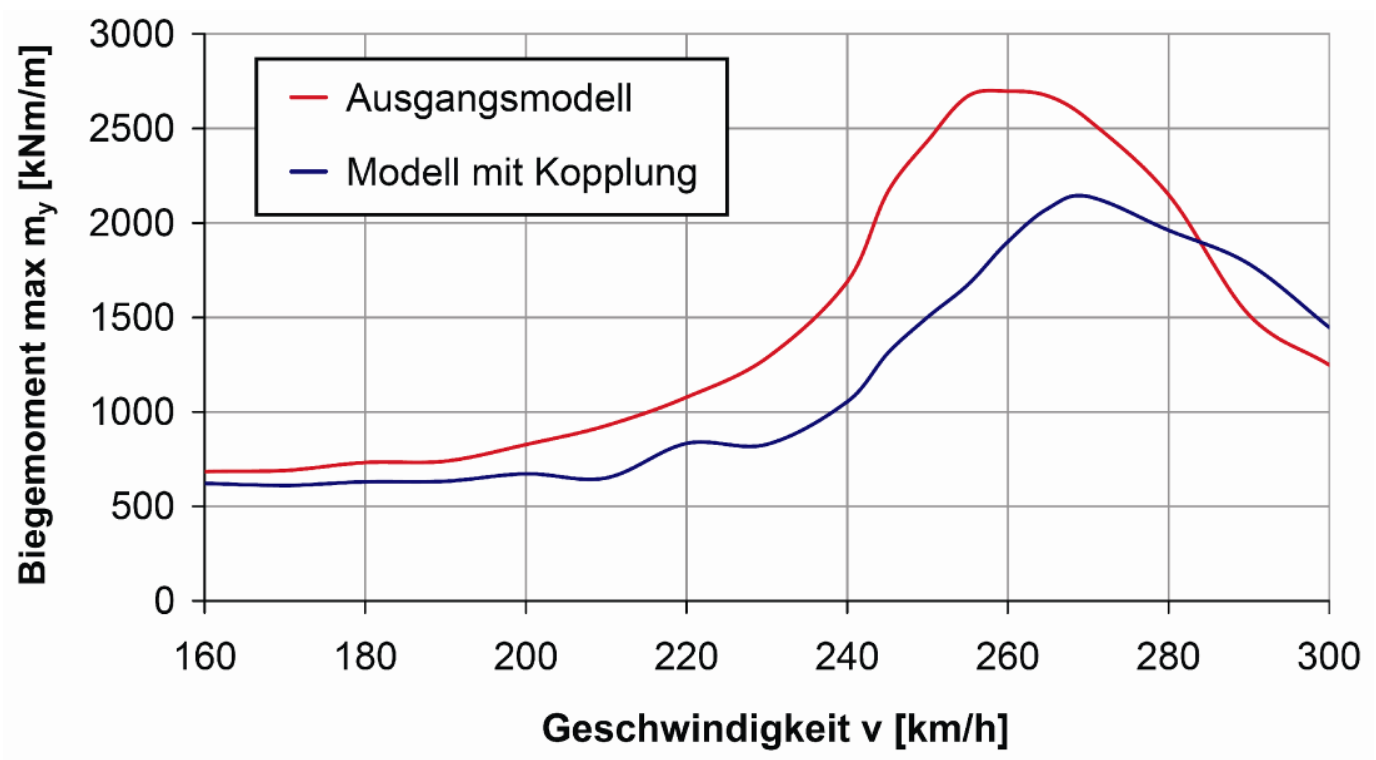

Bild 5.13: Maximale Feldmomente in Abhängigkeit der Zuggeschwindigkeit (ICE-1-Überfahrten) für unterschiedliche numerische Modelle der Brücke Erfttalstraße

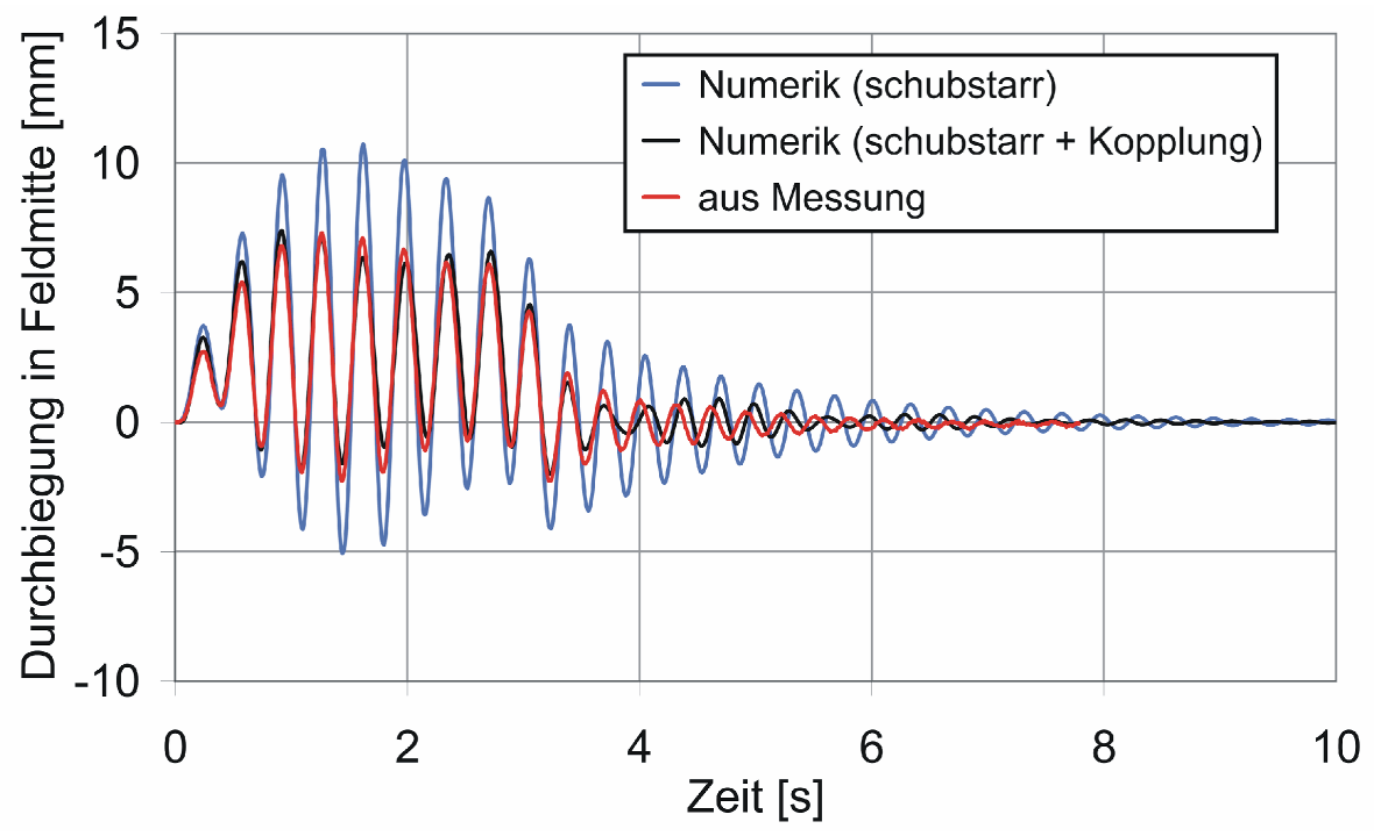

Bild 5.14: Gemessene und berechnete Durchbiegungsverläufe am gekoppelten System mit und ohne Berücksichtigung der Kopplung (ICE-3-Überfahrten mit v $=245 \mathrm{~km} / \mathrm{h}$ ) 
Abschließend wurde das unter Abschnitt 5.2 beschriebene Modell mit schubstarr angeschlossenen Ausbauelementen dahingehend erweitert, dass sowohl die Koppelwirkung durch den Ballast als auch der Einfluss aus Schotter und Gleisen entsprechend Abschnitt 5.3 berücksichtigt wurden. Die numerisch berechneten Durchbiegungen in Feldmitte zeigen nun eine deutlich verbesserte Annäherung an die messtechnisch ermittelten Werte (Bild 5.14) wobei anzumerken ist, dass die numerisch ermittelten Durchbiegungen teilweise etwas kleiner als die Messwerte sind. Dies lässt vermuten, dass die Steifigkeit in der numerischen Berechnung geringfügig überschätzt bzw. die Dämpfung während der Zugüberfahrt unterschätzt wurde. 


\section{Schlussfolgerungen und Vorschläge für die Bemessung}

In der Richtlinie 804 [8] wird die Diskrepanz zwischen theoretisch ermittelten und tatsächlich vorhandenen Eigenfrequenzen bei WIB-Brücken kleiner und mittlerer Stützweiten bereits angesprochen. Als Konsequenz wird in Abschnitt 2 von Modul 804.3301-A01 empfohlen, die folgenden Einflüsse bei der Berechnung der Eigenfrequenzen zu berücksichtigen:

- Einfluss des Gleises auf die Steifigkeit des Gesamtsystems (besonders bei kleinen Stützweiten)

- Berücksichtigung der realen Auflagerbedingungen, die sich von den idealgelenkigen Lagern unterscheiden können

Ergänzend werden in [8] Möglichkeiten zur Umsetzung dieser Empfehlungen bei der Systemmodellierung beschrieben.

Im Rahmen der numerischen Studien wurden die angegebenen Empfehlungen umgesetzt (vgl. Abschnitt 5.3 zum Einfluss des Gleises sowie Abschnitt 5.4 zum Einfluss der Lagerung). Allerdings wurde dabei festgestellt, dass sowohl der Einfluss des Gleises als auch der Einfluss der Lagerung auf die Eigenfrequenzen gering ist. Die Empfehlungen der Ril 804 führen also noch nicht zu der gewünschten Verbesserung der numerischen Ergebnisse und bedürfen nach Meinung des Verfassers der Ergänzung.

Bei den Untersuchungen hat sich herausgestellt, dass der Steifigkeitsbeitrag von Kappe und Schutzschicht von besonderer Relevanz für das dynamische Verhalten der WIB-Überbauten ist. Werden Kappe und Schutzschicht bei der Steifigkeitsberechnung unter Berücksichtigung einer schubstarren Verbindung mit dem Überbau rechnerisch in Ansatz gebracht, so ergeben sich Eigenfrequenzen und maximale Beanspruchungen bei Überfahrtsimulationen, die eine deutliche Annäherung an die realen Verhältnisse darstellen und dennoch auf der sicheren Seite liegen. Dieser Effekt der zusätzlichen Steifigkeit wird als maßgebend für die Fehleinschätzung der Biegeeigenfrequenzen angesehen. $\mathrm{Er}$ tritt verstärkt bei Brücken mit kleinen Stützweiten auf.

Im Rahmen der numerischen Simulation von Zugüberfahrten wurde weiterhin festgestellt, dass sich alle untersuchten Effekte prinzipiell ähnlich abbilden lassen, und zwar durch eine Erhöhung der globalen Biegesteifigkeit - in einem einfachen numerischen Modell beispielsweise durch die Erhöhung des VergleichsE-Moduls. 
Ein Vorschlag zur Berücksichtigung des Einflusses baulicher Randbedingungen wäre daher die Einführung einer effektiven Steifigkeit für die dynamische Bemessung. In Bild 6.1 sind die aus den Messwerten abgeleiteten effektiven Steifigkeiten $\mathrm{El}_{\text {eff }}$ (vgl. Abschnitt 3.1.2) in Abhängigkeit von den rechnerischen Steifigkeiten $\mathrm{El}_{\text {cal }}$ aufgetragen.

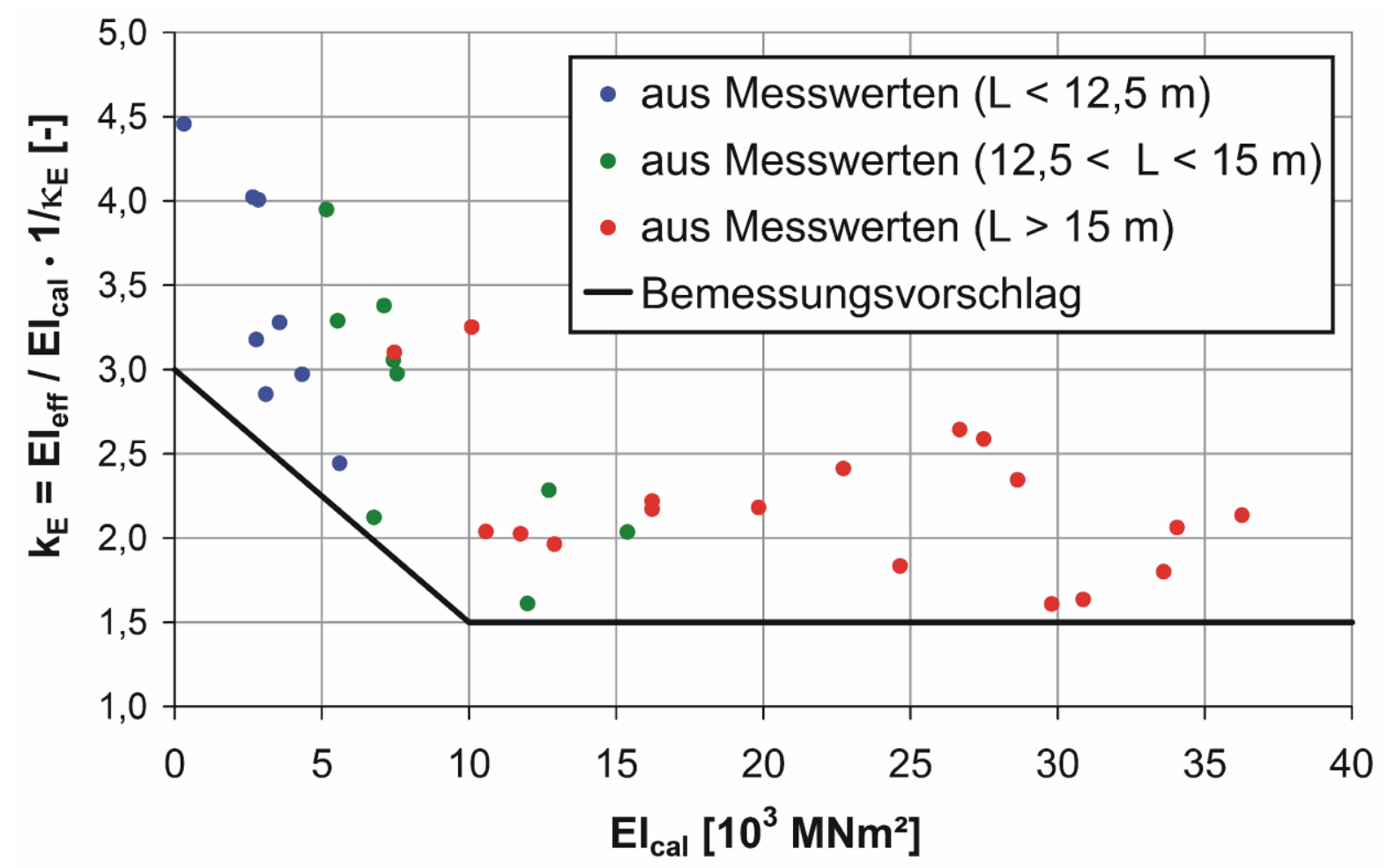

Bild 6.1: Vorschlag für einen Korrekturfaktor $k_{E}$ zur Ermittlung einer effektiven Steifigkeit für die dynamische Bemessung; Gegenüberstellung mit Messwerten

Trägt man in das Diagramm eine untere Grenze der effektiven Steifigkeiten unter Einhaltung eines Sicherheitsabstandes ein, so kann diese Grenze zur Definition eines Korrekturfaktors $k_{E}$ herangezogen werden, mit dem sich aus den rechnerischen Steifigkeiten durch Multiplikation effektive Steifigkeiten berechnen lassen, vgl. Gl. (10). Als Bemessungsvorschlag wurde in Bild 6.1 ein Verlauf für den Korrekturfaktor $k_{E}$ gewählt, so dass dieser bis zu einer rechnerischen Steifigkeit von $10000 \mathrm{kNm}^{2}$ linear von $\mathrm{k}_{\mathrm{E}}=3,0$ auf $\mathrm{k}_{\mathrm{E}}=1,5$ abnimmt und für größere Steifigkeiten konstant bei $k_{E}=1,5$ bleibt. Mit diesem Verlauf wird den Untersuchungsergebnissen Rechnung getragen, die gezeigt haben, dass die Fehleinschätzung von $\mathrm{n}_{0}$ umso größer ist, je kleiner Stützweite bzw. Querschnittssteifigkeit sind. Dieser Zusammenhang wird im Diagramm durch die unterschiedliche Farbgebung der eingetragenen Messergebnisse veranschaulicht. Der Knick des Korrekturfaktorverlaufs liegt in einem Steifigkeitsbereich, der charakteristisch für Brücken mit Stützweiten von etwa 12,5 bis $15 \mathrm{~m}$ ist. Stützweiten 
dieser Größenordnung wurden in Abschnitt 3.1.2 als Grenze identifiziert, ab der die Differenz zwischen gemessener und rechnerischer Eigenfrequenz deutlich abnimmt.

Weiterhin wird vorgeschlagen, einen Beiwert $\kappa_{E}$ bei der Bestimmung der effektiven Steifigkeit einzuführen, mit dem unterschieden wird, ob es sich bei den untersuchten Brücken um Bestands- oder Neubauten handelt. Für Neubauten ließe sich dadurch ein zusätzlicher Abstand von $\mathrm{k}_{\mathrm{E}} \mathrm{zu}$ den aus Messungen abgeleiteten Erfahrungswerten berücksichtigen.

$$
E I_{\text {eff }}=E I_{c a l} \cdot k_{E} \cdot \kappa_{E}
$$

mit

$$
\begin{gathered}
\kappa_{E}=\quad 0,9 \text { (Neubauten) } \\
1,0 \text { (Bestand })
\end{gathered}
$$

Der Korrekturfaktor führt zu einer verbesserten Einschätzung der Grundeigenfrequenz $\mathrm{n}_{0}$. Dadurch können kritische Geschwindigkeiten verschiedener Zugtypen zuverlässiger vorhergesagt und Resonanzeffekte realistischer erfasst werden. Hingegen können Effekte, die keinen nennenswerten Einfluss auf die Eigenfrequenz, jedoch signifikante Auswirkungen auf maximale Beanspruchungen bei Überfahrten haben, mit dem Korrekturfaktor nicht gänzlich abgebildet werden. So führen die Kopplung durch den Schotter und der Einfluss des Gleises zwar zu einer deutlichen Abminderung der Bauwerksbeanspruchungen, nicht aber zu einer nennenswerten Eigenfrequenzänderung. Die Auswirkungen dieser baulichen Randbedingungen spiegeln sich somit nicht in den "gemessenen" effektiven Steifigkeiten wider, auf denen der Ansatz der Korrekturfaktoren basiert. Um diese Effekte dennoch zu berücksichtigen, kann beispielsweise eine Modellierung gemäß den Abschnitten 5.3 und 5.5 vorgenommen werden. Allerdings muss hier betont werden, dass dieses Vorgehen im Rahmen einer dynamischen Bemessung nicht zugelassen werden darf, da eine Kopplung durch den Schotter nicht für die gesamte Nutzungszeit angenommen werden kann. Insbesondere während Wartungsarbeiten kann eine zeitweise einseitige Entfernung von Schotterschichten erfolgen, so dass dann die beschriebene Koppelwirkung entfällt. Bei zweigleisigen Überbauten erübrigt sich die Modellierung der Koppelwirkung. Bei Verwendung eines zweigleisigen Modells wird die aus der Lastquerverteilung resultierende Minderbeanspruchung der befahrenen Überbauhälfte direkt berücksichtigt.

Hinsichtlich der Dämpfung von WIB-Brücken haben die Messergebnisse gezeigt, dass die nach Norm anzusetzenden Dämpfungswerte deutlich geringer 
sind als tatsächlich vorhanden. Im Rahmen der dynamischen Bemessung führt die niedrige rechnerische Dämpfung allerdings nicht wie im Fall der Steifigkeitsabweichungen zu qualitativ unzuverlässigen Ergebnissen, sondern lediglich zu einer verringerten Abminderung der maximalen Schwingungsamplituden bei Überfahrtsimulationen. Die normativen Dämpfungsvorgaben gewährleisten somit eine zusätzliche Sicherheit. Zur Anpassung dieser Vorgaben besteht daher aus Sicherheitsüberlegungen keine Dringlichkeit, jedoch ist eine Anpassung aus wirtschaftlichen und baukulturellen Erwägungen zu überdenken.

Konkret wird folgende Vorgehensweise für eine verbesserte dynamische Bemessung von WIB-Brücken vorgeschlagen:

- Berechnung der Grundeigenfrequenz $\mathrm{n}_{0, \text { eff }}$ unter Ansatz einer effektiven Steifigkeit $\mathrm{El}_{\text {eff. }}$ Diese wird unter Verwendung des Korrekturfaktors $\mathrm{K}_{E}$ gemäß Bild 6.1 nach Gl. (10) ermittelt.

- Prüfung, ob eine explizite dynamische Berechnung erforderlich ist, unter Verwendung von $\mathrm{n}_{0, \text { eff }}$.

- Im Falle einer expliziten dynamischen Berechnung werden die Einwirkungen für die Nachweise im ULS (Schnittgrößen, Auflagerreaktionen, etc.) sowie die Bauwerksreaktionen für die Nachweise im SLS (Beschleunigungen, etc.) unter Berücksichtigung von $\mathrm{El}_{\text {eff }}$ berechnet.

- Auf der Widerstandseite wird nach wie vor nur der nackte Überbauquerschnitt berücksichtigt. Eine mögliche, aus baulichen Randbedingungen resultierende zusätzliche Tragfähigkeit wird nicht in Ansatz gebracht.

- Beibehaltung der bisherigen normativen Dämpfungsvorgaben.

Vorbedingung für dieses Verfahren ist eine bauliche Durchbildung, insbesondere eine Ausbildung von Randkappe und Schutzschicht, die der Regelausführung der Deutschen Bahn entspricht. Die dynamischen Analysen würden durch eine normativ geregelte Steifigkeitsmodifikation an Qualität und Aussagekraft gewinnen. Dem planenden Ingenieur würde eine effektive Bemessungshilfe bereitgestellt, um die Auswirkungen von Zugüberfahrten realistischer abzuschätzen. Durch den Sicherheitsabstand zwischen Korrekturfaktoren und Erfahrungswerten aus Messungen wird gewährleistet, dass die Ergebnisse auf der sicheren Seite liegen, d.h. dass die kritischen Geschwindigkeiten unterschätzt und die maximalen Beanspruchungen überschätzt werden. Darüber hinaus bieten die konservativen Dämpfungsvorgaben eine zusätzliche Sicherheit.

Die dargestellte Vorgehensweise wurde im Rahmen einer gemeinsamen zweiteiligen Publikation mit der Deutschen Bahn abgestimmt [46] [47]. 


\section{Berechnungsbeispiel}

\subsection{Vorbemerkungen}

Um zu zeigen, wie sich die im vorangegangenen Abschnitt angeführten Verbesserungsvorschläge zur dynamischen Bemessung von WIB-Eisenbahnbrücken rechnerisch umsetzen lassen, wird das Vorgehen an einem Beispiel demonstriert. Als Beispiel wurde die WIB-Eisenbahnbrücke EÜ Erfttalstraße ausgewählt (siehe Bild 7.1) da diese Brücke zum einen unter dynamischen Gesichtspunkten von der Deutschen Bahn als problematisch eingeschätzt wurde (vgl. Abschnitt 3.2.1) und ihre dynamische Untersuchung besonders ergiebig im Hinblick auf Konsequenzen aus unterschiedlichen Modellierungsansätzen ist. Zum anderen besteht die Möglichkeit des Vergleichs mit Messergebnissen aus dem Bauwerks-Monitoring, das in Abschnitt 3.2 eingehend beschrieben wurde.

Die Spannweite der Brücke beträgt 24,6 m. Es handelt sich um zwei eingleisige Überbauten mit durchgängiger Schotterschicht. Bei Verwendung von HEM 1000 Walzprofilen beträgt die Querschnittshöhe etwa 1,10 m. Einzelheiten zur baulichen Durchbildung sind Abschnitt 3.2 zu entnehmen.

Im Rahmen dieses Beispiels werden im Sinne einer dynamischen Bemessung für den Hochgeschwindigkeitsverkehr mit $v \geq 200 \mathrm{~km} / \mathrm{h}$ detaillierte Berechnungen durchgeführt, um eine Anregung durch Zugverkehr numerisch zu untersuchen. Die Berechnungen werden exemplarisch an Überbau 2 (vgl. hierzu Bild 7.1c) der Erfttalbrücke vorgeführt. Dabei wird den Berechnungen die Belastung durch einen Hochgeschwindigkeitszug vom Typ ICE-3 zugrunde gelegt, der planmäßig auf der Strecke Köln-Aachen verkehrt, so dass Messdaten zu Brückenüberfahrten von diesem Typ vorliegen und ein Vergleich mit den numerischen Ergebnissen vorgenommen werden kann.

Im Folgenden wird zunächst das Vorgehen bei der Tragwerksmodellierung beschrieben. Ausgangspunkt ist dabei ein numerisches Modell, das in dieser Form als Muster für die derzeit übliche Bemessungspraxis herhalten kann. Bei der Steifigkeitsermittlung wird ausschließlich der nackte Querschnitt des befahrenen Überbaus berücksichtigt, so dass die Steifigkeit konservativ, d. h. auf der sicheren Seite liegend abgebildet wird.

Den Bemessungsvorschlägen aus Abschnitt 6 folgend wird anschließend unter Ansatz einer effektiven Steifigkeit nach Bild 6.1 ein zweites Modell entwickelt, mit dem eine relativ genaue Annäherung an die tatsächliche Steifigkeit erzielt wird 
a)

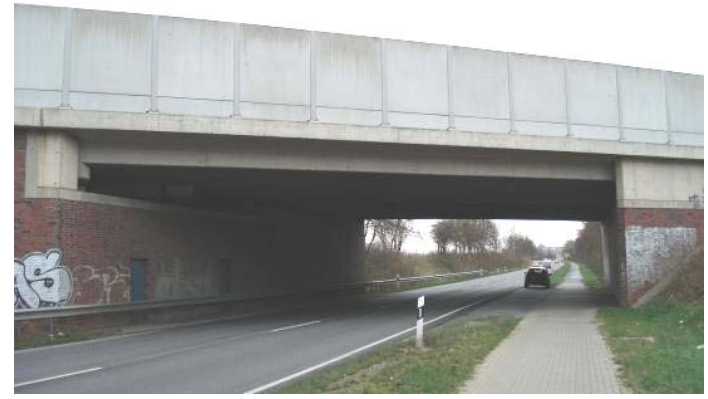

b)

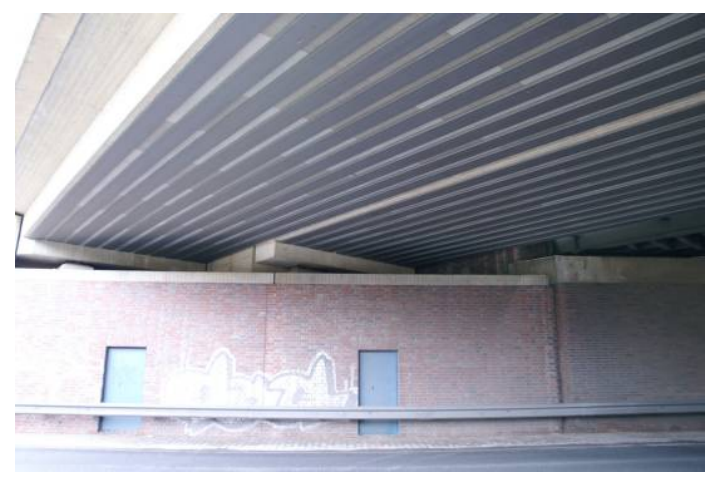

c)

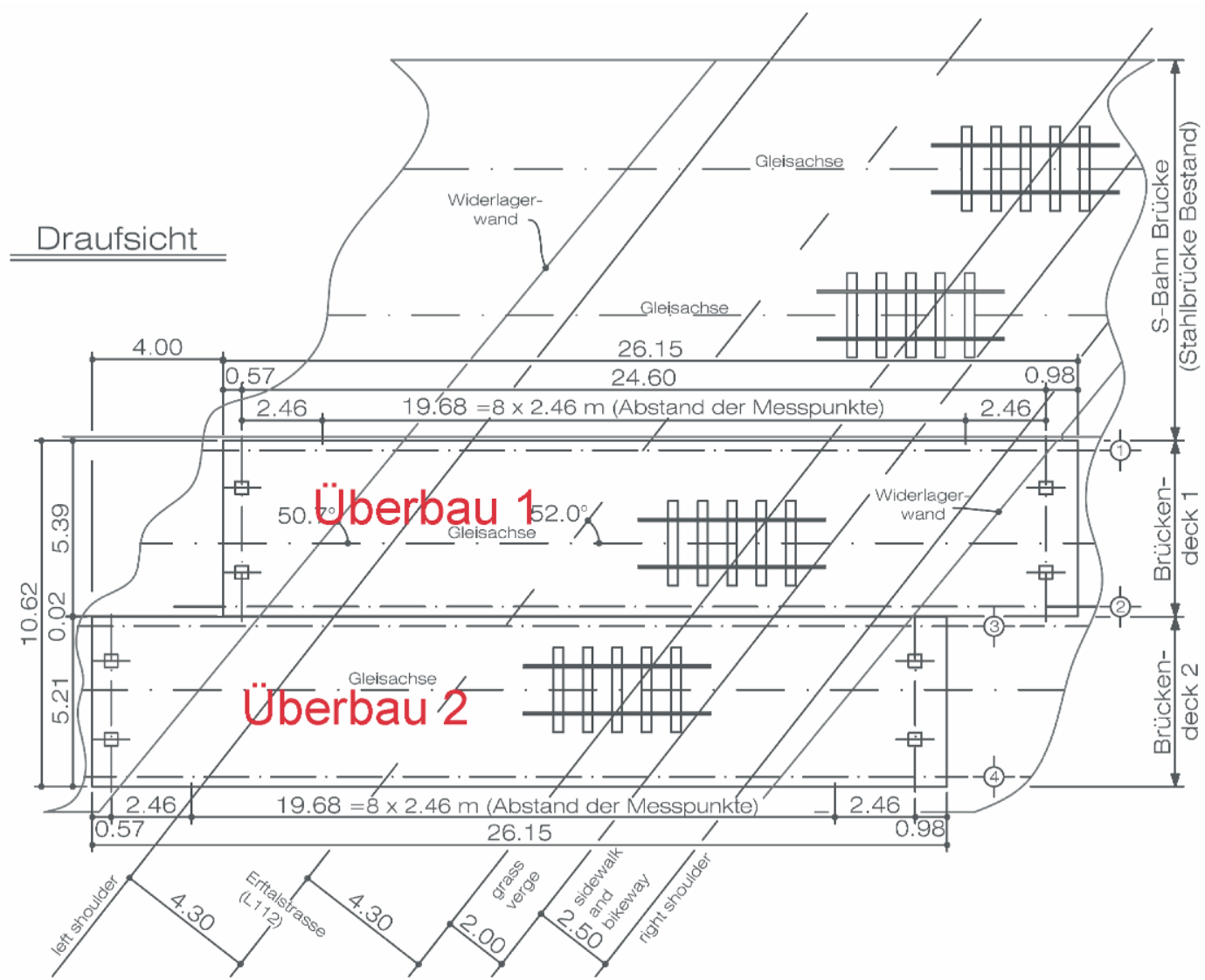

Bild 7.1: Beispielbrücke EÜ Erfttalstraße a) Ansicht Erfttalbrücke b) Untersicht Erfttalbrücke c) Übersichtsplan mit Nummerierung der beiden Überbauhälften

Mit dem dritten numerischen Modell schließlich wird zusätzlich noch der Einfluss der durchgehenden Schotterschicht berücksichtigt, indem die andere Überbauhälfte ebenfalls modelliert und die Koppelwirkung durch den Schotter, den Untersuchungsergebnissen aus den Abschnitten 4 und 5.5 folgend, abgebildet wird.

Die drei Modelle werden anschließend dazu verwendet, ICE-3-Überfahrten zu simulieren, wobei als Fahrzeugmodell analog zu den numerischen Berechnungen in Abschnitt 5 das Modell der „bewegten Einzellasten“ zur Anwendung 
kommt. Abschließend folgt eine Diskussion der Simulationsergebnisse, die den Messwerten aus der in Abschnitt 3.2 behandelten Monitoringkampagne gegenübergestellt werden.

\subsection{Tragwerksmodellierung}

\subsubsection{Allgemeines}

Zur Modellierung der WIB-Brücke Erfttalstraße wird das FE-Programm InfoCAD [27] verwendet, mit dem Überfahrtsimulationen nach dem NewmarkIntegrationsverfahren durchgeführt werden können. Zur Definition der Lasten aus Zugverkehr steht eine programminterne Bibliothek zur Verfügung, die unter anderem auch den ICE-3-Zugtyp enthält.

Die WIB-Platten werden mit 4-Knoten-Plattenelementen modelliert, wobei die orthotropen Eigenschaften berücksichtigt werden, d. h. die Platte besitzt unterschiedliche Steifigkeiten in Längs- und Querrichtung. Die Steifigkeit in Längsrichtung ist aufgrund der in dieser Richtung verlaufenden Stahlträger deutlich größer. Der Modellierung wird die in Bild 7.2 dargestellte Querschnittsgeometrie zugrunde gelegt. Auf eine Modellierung der Endquerträger wird aufgrund des zu vernachlässigenden Einflusses verzichtet. Die Lagerung der Platte erfolgt durch starre Linienlager.

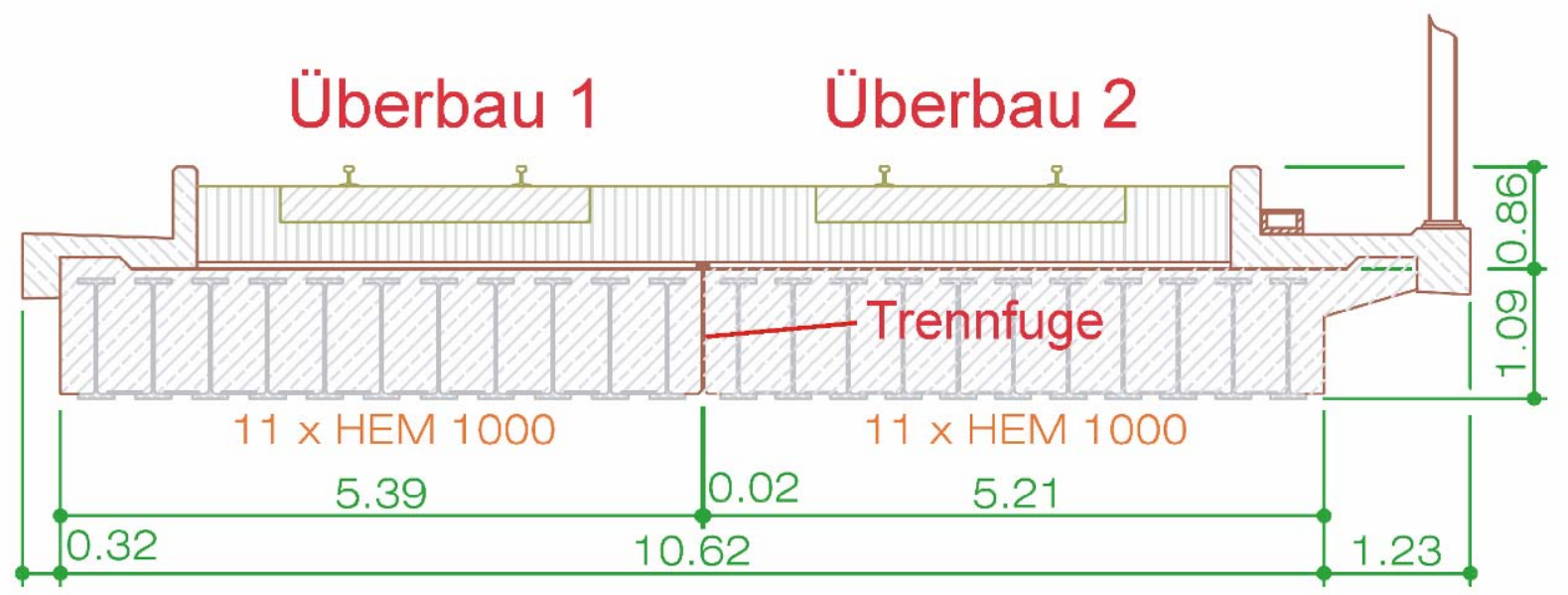

Bild 7.2: Querschnitt des Überbaus EÜ Erfttalstraße

\subsubsection{Modell 1: Nackter WIB-Querschnitt}

Mit dem ersten Modell wird ausschließlich der nackte WIB-Querschnitt von Überbau 2 abgebildet, d. h. ohne Berücksichtigung zusätzlicher baulicher Elemente, welche die Überbausteifigkeit beeinflussen. 
Zunächst wird die Steifigkeit der WIB-Platte in Haupttragrichtung ermittelt. Das ideelle Trägheitsmoment $I_{i}$ des Verbundquerschnitts lässt sich nach Ril 804 (804.4302 A 01) [8] gemäß nachfolgenden Gleichungen berechnen. Es wurden hier die in der Ril 804 verwendeten Bezeichnungen übernommen, vgl. Bild 7.3. Als Vergleichs-E-Modul bei der Berechnung der Verbundquerschnittswerte wird der Beton-E-Modul gewählt. Es wird angenommen, dass der Betonquerschnitt ungerissen ist. Zudem wird die obere Längsbewehrung vernachlässigt, da sie nur einen geringen Einfluss auf Querschnittssteifigkeit besitzt.

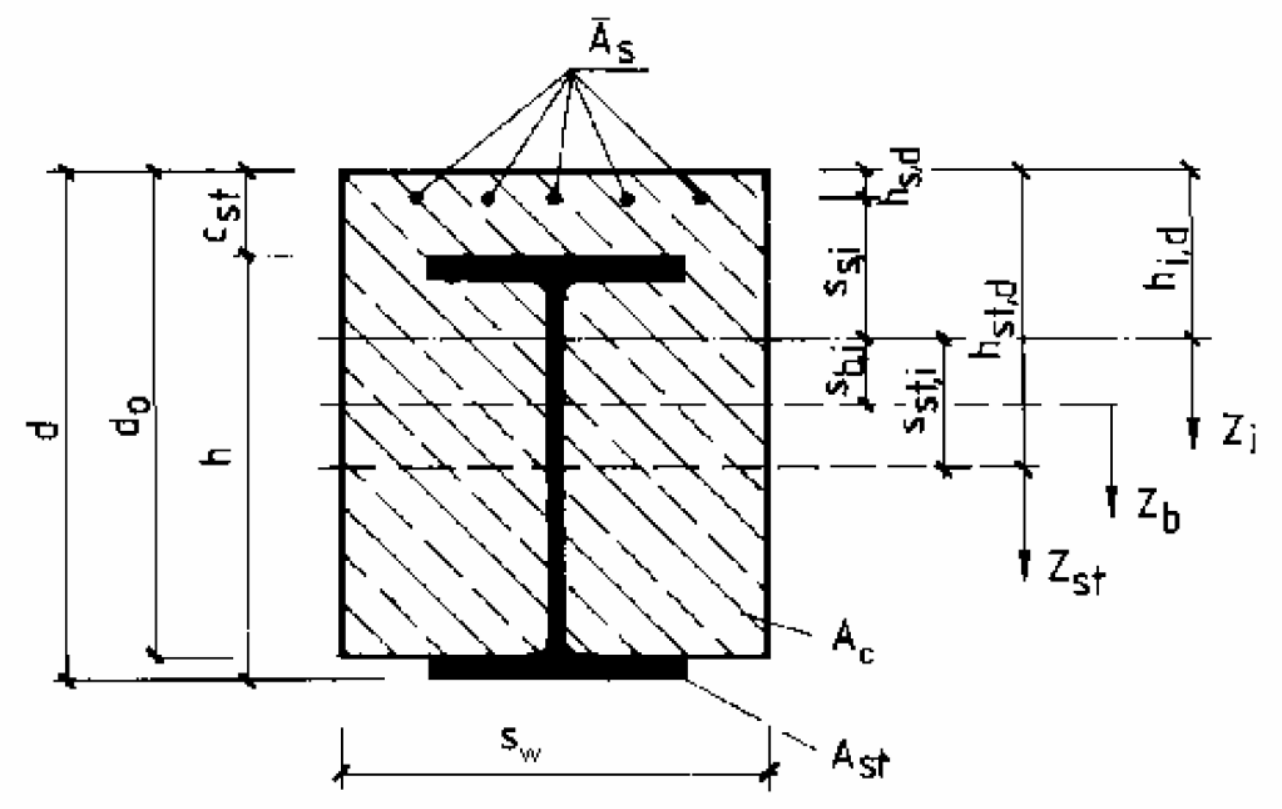

Bild 7.3: Bezeichnung der geometrischen Größen des gesamten Verbundquerschnitts aus [8]

$$
\begin{aligned}
& A_{c}=s_{w} \cdot d_{0} \\
& h_{s t, d}=\frac{h}{2}+c_{s t} \\
& h_{i, d}=\frac{(n-1) \cdot A_{s t} \cdot h_{s t, d}+A_{c} \cdot 0,5 \cdot d_{0}}{(n-1) \cdot A_{s t}+A_{c}} \\
& s_{s t, i}=h_{s t, d}-h_{i, d} \\
& s_{b, i}=0,5 \cdot d_{0}-h_{i, d} \\
& I_{i}=(n-1) \cdot\left(I_{s t}+A_{s t} \cdot s_{s t, i}^{2}\right)+A_{c} \cdot s_{b, i}^{2}+\frac{s_{w} \cdot d_{0}^{3}}{12}
\end{aligned}
$$


Bei der Berechnung des ideellen Trägheitsmomentes $I_{i}$ wird zunächst nur ein Abschnitt des Gesamtquerschnitts der Breite $\mathrm{s}_{\mathrm{w}}$ (Abstand der Stahlträger untereinander) betrachtet. Die in die Rechnung eingehenden geometrischen Größen für den Überbau Erfttalstraße sind in Bild 7.4 dargestellt.
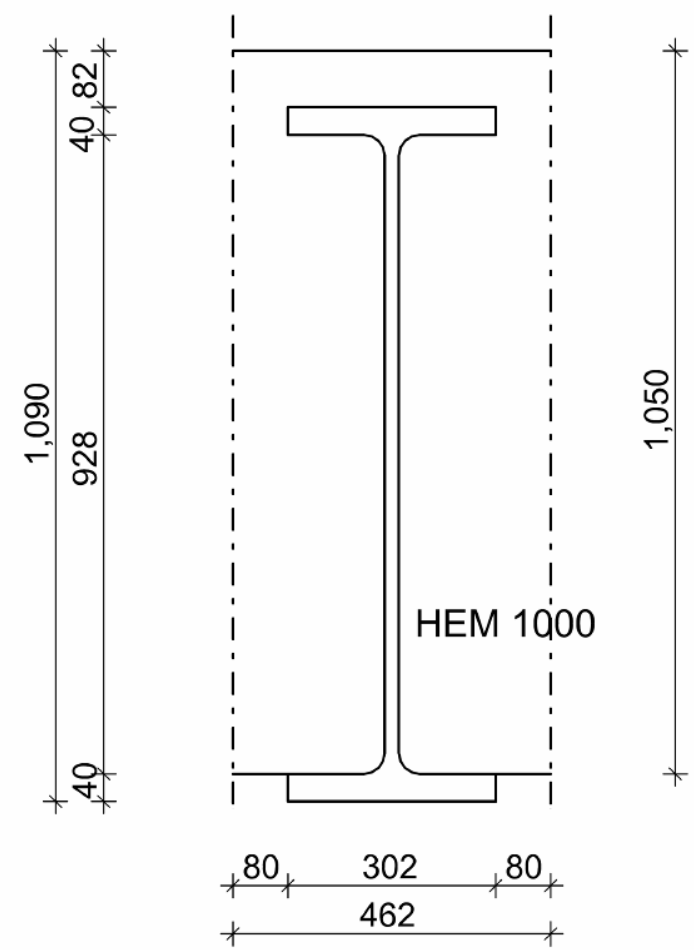

\section{Bild 7.4: Ausschnitt WIB-Platte EÜ Erfttalstraße mit relevanten Abmes- sungen}

Zur Bestimmung des ideellen Gesamtträgheitsmomentes ist das Trägheitsmoment $I_{i}$ des Einzelabschnittes noch mit der Anzahl der einbetonierten Walzträger $\mathrm{i}_{\text {Träger }}$ zu multiplizieren.

$$
I_{W I B, i}=I_{i} \cdot i_{\text {Träger }}
$$

Um dieses Trägheitsmoment dem Plattenmodell zuzuweisen, wird unter Berücksichtigung der Plattenbreite $b_{\text {WIB }}$, die der tatsächlichen Breite des WIBÜberbaus entspricht, eine virtuelle Plattenhöhe $h_{\text {virt }}$ gemäß nachfolgender Gleichung berechnet.

$$
h_{\text {virt }}=\sqrt[3]{\frac{12 \cdot I_{W I B, i}}{b_{W I B}}}
$$

Außerdem wird eine virtuelle Wichte unter Berücksichtigung aller ständigen Lasten bestimmt, damit die Bauwerksmasse durch InfoCAD berechnet werden kann. 


$$
\gamma_{\text {virt }}=\frac{G_{\text {ges }}}{b_{W I B} \cdot h_{\text {virt }}}
$$

In Tabelle 7.1 wird exemplarisch vorgeführt, wie sich die Querschnittssteifigkeit der WIB-Platte und die Kennwerte zur Modellierung der virtuellen Platte mit InfoCAD berechnen lassen.

\section{Tabelle 7.1: Berechnung der Querschnittssteifigkeit und der Kennwerte zur Modellierung einer virtuellen Platte mit InfoCAD}

Überbau 2 - Erfttalbrücke

(Querschnittsberechnung nach Ril 804.4302 A01)

Querschnittsgeometrie

\begin{tabular}{lcrl}
\hline Gesamthöhe & $\mathrm{d}$ & 109 & $\mathrm{~cm}$ \\
Betonhöhe & $\mathrm{d}_{0}$ & 105 & $\mathrm{~cm}$ \\
Betonbreite & $\mathrm{S}_{\mathrm{w}}$ & 46,2 & $\mathrm{~cm}$ \\
Obere Betondeckung & $\mathrm{C}_{\mathrm{st}}$ & 8,2 & $\mathrm{~cm}$ \\
Höhe Stahlprofil & $\mathrm{h}$ & 100,8 & $\mathrm{~cm}$ \\
Fläche Stahlprofil & $\mathrm{A}_{\mathrm{st}}$ & 444 & $\mathrm{~cm}^{2}$ \\
Trägheitsmoment Stahlprofil & $\mathrm{I}_{\mathrm{st}}$ & 722300 & $\mathrm{~cm}^{4}$ \\
Anzahl der Walzträger & $\mathrm{i}_{\text {Träger }}$ & 11 & - \\
& & & \\
Material & & 210000 & $\mathrm{~N} / \mathrm{mm}^{2}$ \\
\hline E-Modul Stahl & $\mathrm{E}_{\mathrm{a}}$ & 35000 & $\mathrm{~N} / \mathrm{mm}^{2}$ \\
E-Modul Beton & $\mathrm{E}_{\mathrm{cm}}$ & 6 & -
\end{tabular}

Massen und Gewichte

\begin{tabular}{lcrl}
\hline Eigengewicht WIB-Platte & $\mathrm{G}_{\mathrm{eg}}$ & 171,7 & $\mathrm{kN} / \mathrm{m}$ \\
Ausbaulast WIB-Platte & $\mathrm{G}_{\text {aus }}$ & 100,8 & $\mathrm{kN} / \mathrm{m}$ \\
Gesamtgewicht WIB-Platte & $\mathrm{G}_{\mathrm{ges}}$ & 272,5 & $\mathrm{kN} / \mathrm{m}$ \\
Gesamtmasse & $\mathrm{M}_{\mathrm{ges}}$ & 27,78 & $\mathrm{t} / \mathrm{m}$ \\
Berechnung Querschnittssteifigkeit & & & \\
\hline Betonfläche & $\mathrm{A}_{\mathrm{c}}$ & 4851 & $\mathrm{~cm}^{2}$ \\
Abstand & $\mathrm{h}_{\mathrm{st}, \mathrm{d}}$ & 58,60 & $\mathrm{~cm}$ \\
Lage Schwerpunkt Gesamtquerschnitt & $\mathrm{h}_{\mathrm{i}, \mathrm{d}}$ & 54,42 & $\mathrm{~cm}$ \\
Abstand & $\mathrm{S}_{\mathrm{st}, \mathrm{i}}$ & 4,18 & $\mathrm{~cm}$ \\
Abstand & $\mathrm{S}_{\mathrm{b}, \mathrm{i}}$ & $-1,92$ & $\mathrm{~cm}$ \\
Trägheitsmoment Gesamtquerschnitt & $\mathrm{I}_{\mathrm{i}}$ & 8125028 & $\mathrm{~cm}$ \\
Trägheitsmoment WIB-Platte & $\mathrm{I}_{\mathrm{WIB,i}}$ & 0,893753 & $\mathrm{~m}$ \\
Biegesteifigkeit WIB-Platte & $\mathrm{El}_{\mathrm{WIB}}$ & 31281 & $\mathrm{MNm}$ \\
& & & \\
Kenngrößen virtuelle Platte & & & \\
\hline Gesamtbreite der Brücke & $\mathrm{b}_{\text {WIB }}$ & 5,21 & $\mathrm{~m}$ \\
Höhe virtuelle Platte & $\mathrm{h}_{\text {virt }}$ & 1,272 & $\mathrm{~m}$ \\
Wichte & $\gamma_{\text {virt }}$ & 41,12 & $\mathrm{kN} / \mathrm{m}^{3}$
\end{tabular}


Zur Definition von orthotropen Platteneigenschaften bzw. zur Anpassung der Steifigkeit in Plattenquerrichtung ist in InfoCAD ein Orthotropie-Faktor vorgesehen. Dieser Faktor dient der Abminderung der Elementdicke für die Berechnung der Steifigkeit in lokaler y-Richtung eines Elementes. Es ist also darauf zu achten, wie die lokalen Koordinatensysteme der Plattenelemente ausgerichtet sind. Vor dem Hintergrund, dass bei eingleisigen Überbauten in der Regel keine Lasten quer durch den Plattenquerschnitt geleitet werden, da sich die Verkehrslast bei üblichen Querschnittsabmessungen und einem Lastausbreitungswinkel von $45^{\circ}$ bereits auf den gesamten Überbau verteilt und diesen gleichmäßig beansprucht, wird vereinfacht von einem ungerissenen Querschnitt in Plattenquerrichtung ausgegangen. Damit wird die Querverteilung der Lasten in der numerischen Berechnung sichergestellt. Der Orthotropie-Faktor kann somit sehr einfach nach Gleichung (7-10) unter Verwendung der Betonhöhe $d_{0}$ berechnet werden.

$$
f_{\text {ortho }}=d_{0} / h_{i, d}
$$

Es sind nun alle wesentlichen Parameter zur Generierung von Modell 1 bekannt. Das entsprechende Tragwerksmodell ist abschließend in Bild 7.5 dargestellt.

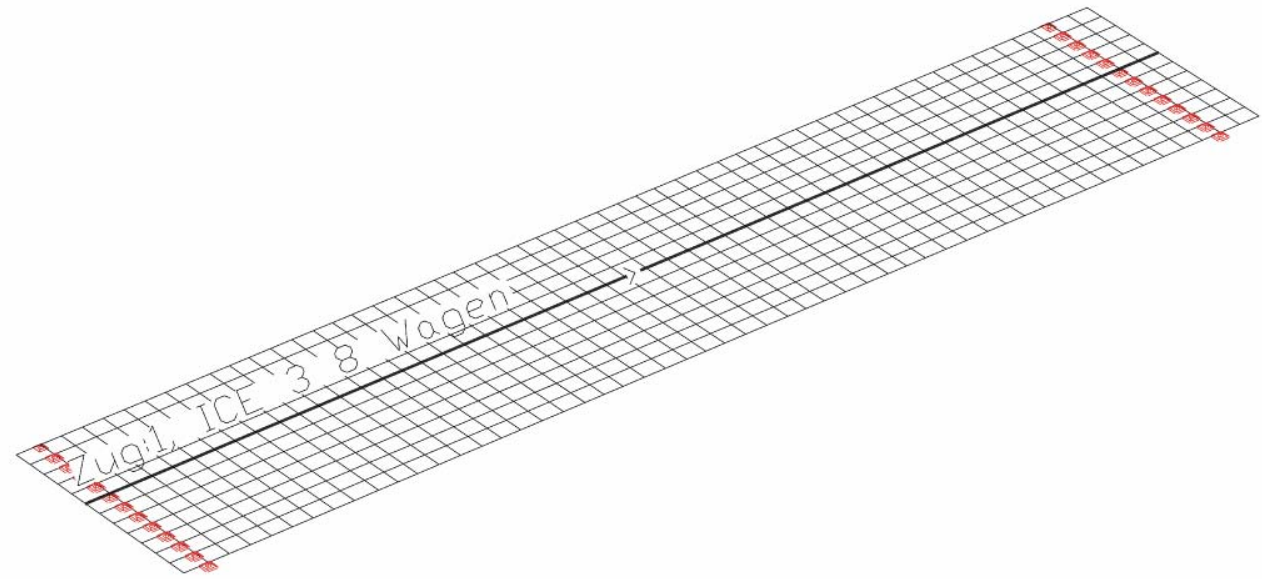

Bild 7.5: Modell 1 zur Tragwerksmodellierung der WIB-Brücke EÜ Erfttalstraße mit Kennzeichnung des Fahrweges

\subsubsection{Modell 2: Steifigkeitsmodifikation mit Korrekturfaktor}

Im nächsten Schritt werden die in Abschnitt 6 vorgetragenen Bemessungsvorschläge berücksichtigt.

Zur Berücksichtigung zusätzlicher Steifigkeitsbeiträge infolge baulicher Randbedingungen wird die rechnerische Steifigkeit von Modell 1 mit Hilfe des in Bild 6.1 angegebenen Korrekturfaktors modifiziert. Um die daraus resultierende Zu- 
satzsteifigkeit im numerischen Modell abzubilden, bieten sich die folgenden beiden Möglichkeiten an:

a) Multiplikation des Vergleichs-E-Moduls mit dem Korrekturfaktor $k_{E}=1,5$ (hier mit $\kappa_{E}=1,0$ )

b) Modifikation der virtuellen Plattendicke gemäß Gleichung (7-11)

$$
h_{\text {virt, neu }}=\sqrt[3]{k_{E} \cdot \kappa_{E}} \cdot h_{\text {virt }}
$$

Im Rahmen dieser Beispielrechnung wurde der Vergleichs-E-Modul unter Verwendung von Methode a von $35.000 \mathrm{MN} / \mathrm{m}^{2}$ auf $52.500 \mathrm{MN} / \mathrm{m}^{2}$ erhöht. Unter der Annahme, dass die zusätzliche Steifigkeit hauptsächlich in Längsrichtung wirksam ist, muss streng genommen noch der Orthotropie-Faktor gemäß nachstehender Gleichung modifiziert werden, um durch die Erhöhung des E-Moduls nicht gleichzeitig auch die Steifigkeit in Plattenquerrichtung zu vergrößern. Allerdings ist der Einfluss dieses Faktors auf die numerischen Ergebnisse vernachlässigbar gering. Er wird hier nur der Vollständigkeit halber angegeben:

$$
f_{\text {ortho, пеu }}=\frac{f_{\text {ortho }}}{\sqrt[3]{k_{E} \cdot \kappa_{E}}}
$$

Weitere Modifikationen von Modell 1 sind nicht notwendig. Die äußere Form von Modell 2 entspricht dem in Bild 7.5 dargestellten Modell 1.

\subsubsection{Modell 3: Zusätzliche Berücksichtigung von Koppelfedern}

Um zusätzlich auch noch die Koppelwirkung durch den Eisenbahnschotter zu berücksichtigen, wird nun ein deutlich komplexeres numerisches Modell der Erfttalbrücke erstellt.

Zunächst wird der zweite Überbau analog zum ersten Überbau auf Grundlage von Abschnitt 7.2.2 bzw. 7.2.3 modelliert. Dabei ist darauf zu achten, dass die Abstände der Randknoten beider Überbauten in der Koppelfuge jeweils übereinstimmen. Einander gegenüberliegende Randknoten bilden damit einzelne Knotenpaare. Eine Schotterkopplung kann dann durch eine Federverbindung dieser Knotenpaare in vertikaler Richtung realisiert werden, vgl. Bild 7.6.

Die Steifigkeit der Federelemente wird in Abhängigkeit von der Schotterhöhe und dem Abstand der Federn bestimmt. Dabei wird den Federelementen ein bilineares Federgesetz gemäß Bild 4.13 zugewiesen. Die Kontaktfläche, die durch ein einzelnes Federelement repräsentiert wird, ergibt sich aus dem Produkt von Schotterhöhe (Annahme hier $0,5 \mathrm{~m}$ ) und mittlerem Abstand der Federelemente (hier ebenfalls $0,5 \mathrm{~m}$ ): 


$$
A_{\text {Kontakt }}=0,5 \mathrm{~m} \cdot 0,5 \mathrm{~m}=0,25 \mathrm{~m}^{2}
$$

Damit berechnet sich die Grenzkraft, die sich bei 2,0 mm Relativverformung in der Koppelfuge einstellt und nicht weiter gesteigert werden kann wie folgt:

$$
F_{\text {grenz }}=4350 \mathrm{kN} / \mathrm{m}^{3} \cdot 0,002 \mathrm{~m} \cdot 0,25 \mathrm{~m}^{2}=2,175 \mathrm{kN}
$$

Diese Grenzkraft wird in InfoCAD zur Definition des nichtlinearen Federgesetzes benötigt. Zur Generierung von Modell 3 stehen nun alle Parameter zur Verfügung. Es ist abschließend in Bild 7.6 dargestellt.

a)

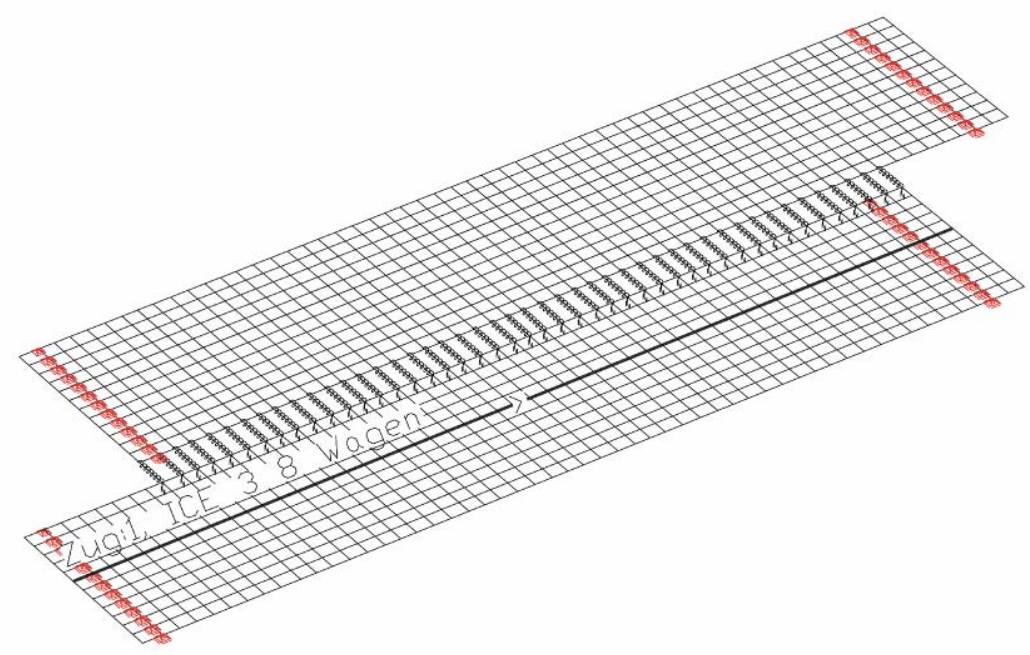

b)

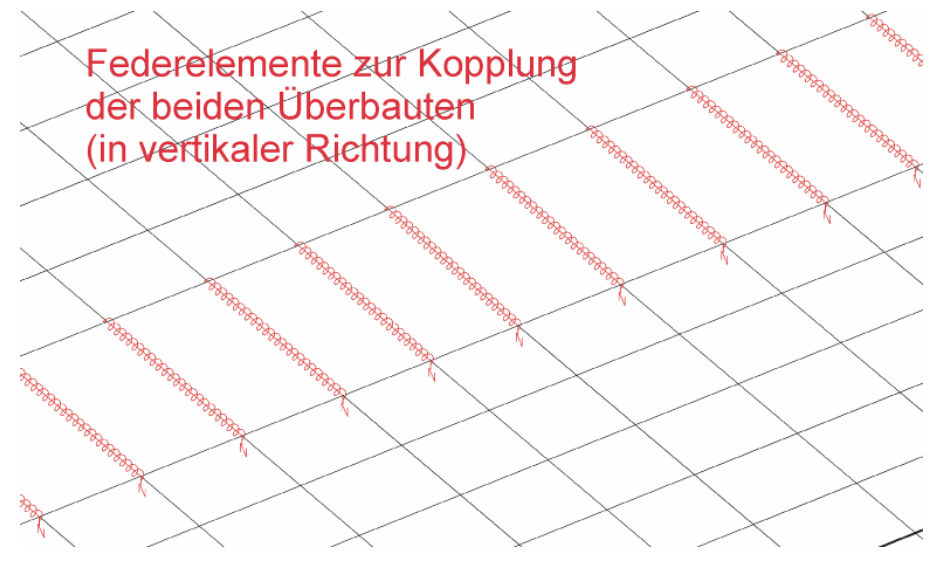

Bild 7.6: Modell 3 zur Tragwerksmodellierung der WIB-Brücke EÜ Erfttalstraße a) Gesamtübersicht mit Kennzeichnung des Fahrweges b) Ausschnitt Koppelfuge mit Federelementen

\section{3 Überfahrtsimulationen}

Unter Verwendung der drei entwickelten Brückenmodelle sollen dynamische Simulationsrechnung mit InfoCAD durchgeführt werden, wobei als Fahrzeugmodell das in InfoCAD implementierte Modell der „bewegten Einzellasten“ ein- 
gesetzt wird. Der auf der HGV-Strecke Köln-Aachen verkehrende ICE-3-Zug besteht aus acht Wagen (Endwagen +6 Mittelwagen + Endwagen), so dass die in InfoCAD voreingestellte ICE-3-Zugkonfiguration, bei der 16 Wagen $(8+8$ Wagen) berücksichtigt werden, entsprechend zu modifizieren ist. Die sich daraus ergebenden Achslasten sind in Tabelle 7.2 angegeben. Diese Achslasten stellen die bewegten unabhängigen Einzellasten dar, die sich mit einer definierten Geschwindigkeit auf dem Tragewerk entlang bewegen.

Tabelle 7.2: Konfiguration der Achslasten beim Hochgeschwindigkeitszug ICE-3 (InfoCAD-Ausgabe)

\begin{tabular}{r|r|r|}
\multicolumn{3}{|c}{ Zugdefinition Benutzerdefiniert } \\
\hline & Abstand [m] & Achslast [kN] \\
\hline 1 & 0,000 & 149,400 \\
2 & 2,500 & 149,400 \\
3 & 17,375 & 148,400 \\
\hline 4 & 19,875 & 148,400 \\
\hline 5 & 24,775 & 166,300 \\
6 & 27,275 & 166,300 \\
7 & 42,150 & 163,300 \\
\hline 8 & 44,650 & 163,300 \\
\hline 9 & 49,550 & 154,700 \\
10 & 52,050 & 154,700 \\
11 & 66,925 & 157,600 \\
12 & 69,425 & 157,600 \\
13 & 74,325 & 143,100 \\
14 & 76,825 & 143,100 \\
15 & 91,700 & 144,100 \\
16 & 94,200 & 144,100 \\
17 & 99,100 & 148,500 \\
18 & 101,600 & 148,500 \\
19 & 116,475 & 142,500 \\
20 & 118,975 & 142,500 \\
21 & 123,875 & 161,800 \\
22 & 126,375 & 161,800 \\
\hline 23 & 141,250 & 159,000 \\
\hline 24 & 143,750 & 159,000 \\
25 & 148,650 & 168,000 \\
26 & 151,150 & 168,000 \\
27 & 166,025 & 170,900 \\
28 & 168,525 & 170,900 \\
29 & 173,425 & 151,700 \\
30 & 175,925 & 151,700 \\
31 & 190,800 & 152,300 \\
32 & 193,300 & 152,300 \\
\hline & & \\
\hline 1 & &
\end{tabular} \mid

Der „Fahrweg“, auf dem sich die Lasten bewegen, kann knotenunabhängig vorgegeben werden und sollte sich möglichst genau in der Gleisachse befinden, vgl. Bild 7.5 bzw. Bild 7.6

Die Systemdämpfung wird gemäß Norm angesetzt, vgl. Bild 2.8. Demnach ergibt sich bei einer Spannweite von 24,6 m ein Wert von 1,6\%, der zur Definition 
der Rayleigh-Dämpfung verwendet wird. Dabei sind zwei Wertepaare vorzugeben, die aus jeweils einer Frequenz mit zugehörigem Dämpfungswert bestehen. Im Rahmen dieser Beispielrechnung wird den ersten beiden SystemEigenfrequenzen der o. a. normative Dämpfungswert zugewiesen. Die Systemmasse wird vom Programm automatisch unter Berücksichtigung der vorgegebenen Wichte ermittelt. Als Zeitschrittweite für das NewmarkIntegrationsschema werden 0,005 s gewählt, vgl. Abschnitt 2.4.3.

\subsection{Ergebnisse und Vergleich mit Messwerten}

Mit den drei Tragwerksmodellen wurde die Systemsteifigkeit schrittweise erhöht. Dies spiegelt sich in der Erhöhung der zugehörigen Grundeigenfrequenzen wider, vgl. Tabelle 7.3. Allerdings wirkt sich, wie bereits in Abschnitt 5.5 erläutert, die Berücksichtigung der Koppelwirkung in Modell 3 nur geringfügig auf die Grundeigenfrequenz aus. Aus den höheren Grundeigenfrequenzen ergeben sich entsprechend Gleichung (2-2) höhere kritische Geschwindigkeiten, bei denen die Brücke in Resonanz angeregt wird. Dieser Zusammenhang kann ebenfalls Tabelle 7.3 entnommen werden.

Tabelle 7.3: Grundeigenfrequenzen der drei Brückenmodelle mit den zugehörigen kritischen Geschwindigkeiten für den ICE-3

\begin{tabular}{lccc} 
Train type & ICE 3 & & \\
\cline { 1 - 2 } $\mathrm{L}_{\text {üP }}[\mathrm{m}]$ & 24,8 & & \\
\hline & Modell 1 & Modell 2 & Modell 3 \\
\cline { 2 - 4 } $\mathrm{f}_{0}[\mathrm{~Hz}]$ & 2,75 & 3,35 & 3,37 \\
\hline $\mathbf{V}_{\text {criti, } 1}[\mathrm{~km} / \mathrm{h}]$ & 246 & 299 & 301 \\
\hline
\end{tabular}

Bei Durchführung der Überfahrtsimulationen macht sich die Kopplung der beiden Überbauten deutlich stärker bemerkbar. Betrachtet man in Bild 7.7 die maximal auftretenden Beschleunigungen, die sich im Rahmen der Simulationsrechnung ergeben, in Abhängigkeit von der Überfahrtgeschwindigkeit, so ergeben sich qualitative Verläufe, die bereits aus Abschnitt 5 bekannt sind. Mit zunehmender Steifigkeit verschiebt sich das Kurvenmaximum nach rechts, d. h. der Maximalwert tritt bei höheren Geschwindigkeiten auf. Während bei den Modellen 1 und 2 keine nennenswerte Änderung des Maximalwerts zu verzeichnen ist, reagiert Modell 3 mit deutlich reduzierten Beschleunigungswerten auf die Zugüberfahrten. 
Um zu überprüfen, wie realistisch die einzelnen Modelle das tatsächliche Systemverhalten abbilden, wird auf Ergebnisse aus dem Bauwerks-Monitoring an der Erfttalbrücke zurückgegriffen. In Abschnitt 3.2 wurden Messergebnisse präsentiert, die aus ICE-3 Überfahrten resultieren. Dabei wurde zwischen Sommerund Winterdaten unterschieden. Im Folgenden werden diese Messergebnisse den numerischen Ergebnissen gegenübergestellt, wobei ausschließlich Überbau 2 betrachtet wird. Insgesamt wurden jeweils rund 100 ICE-Überfahrten ausgewertet, die bei sommer- bzw. winterlichen Witterungsverhältnissen aufgezeichnet wurden, vgl. Abschnitt 3.2.5 und Tabelle 3.4.

In Bild 7.7 sind zunächst die maximal auftretenden Beschleunigungen aufgetragen, die einerseits numerisch berechnet und andererseits an der Erfttalbrücke gemessen wurden. Bezüglich der Sommerdaten liefern alle drei numerischen Modelle auf der sicheren Seite liegende Ergebnisse, wobei das komplexe Modell mit expliziter Berücksichtigung des Koppeleffekts (Modell 3) insbesondere im maximalen Geschwindigkeitsbereich von etwa $250 \mathrm{~km} / \mathrm{h}$ das reale Systemverhalten am besten wiedergibt.

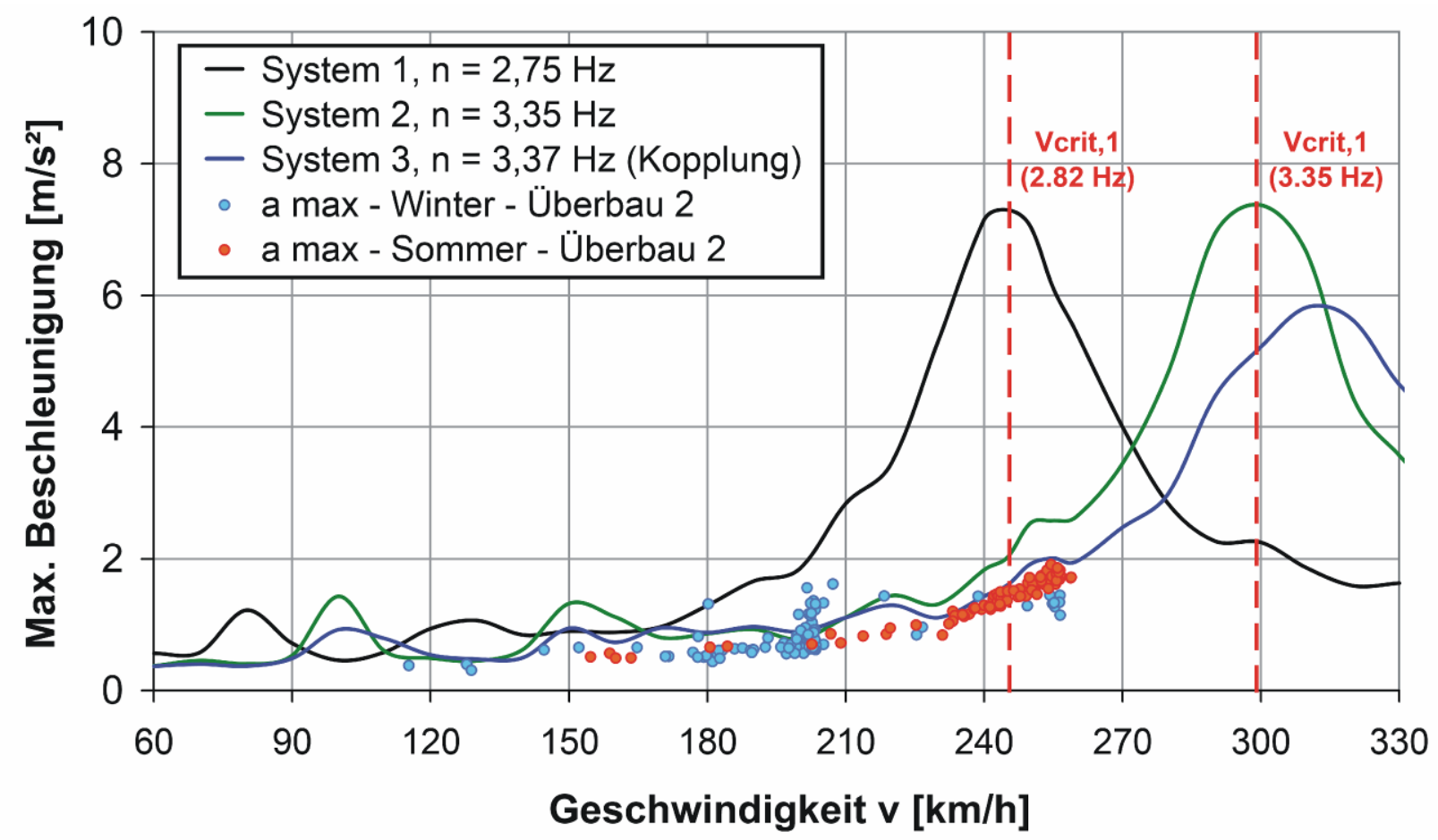

Bild 7.7: Gegenüberstellung von berechneten und gemessenen maximalen Beschleunigungen in Feldmitte (aufgetragen jeweils in Abhängigkeit von der Überfahrtgeschwindigkeit)

Modell 1 liefert eine sehr ungünstige Einschätzung maximal auftretender Beschleunigungen. Gerade im bemessungsrelevanten Geschwindigkeitsbereich um $250 \mathrm{~km} / \mathrm{h}$ sind die Ergebnisse vollkommen unrealistisch. Zulässige Be- 
schleunigungen von $3,5 \mathrm{~m} / \mathrm{s}^{2}$ werden bereits bei einer Geschwindigkeit von etwa $230 \mathrm{~km} / \mathrm{h}$ überschritten, obwohl auch bei deutlich höheren Geschwindigkeiten keine Beschleunigungen größer $2,0 \mathrm{~m} / \mathrm{s}^{2}$ gemessen wurden.

In Bezug auf die Winterdaten fallen die bei etwa $200 \mathrm{~km} / \mathrm{h}$ vereinzelt auftretenden Beschleunigungswerte auf, die deutlich größer als die mit den Modellen 2 und 3 ermittelten Rechenwerte sind. Diese Unregelmäßigkeiten des gemessenen Beschleunigungsspektrums wurden bereits in Abschnitt 3.2.6 diskutiert. Sie wird auf witterungsbedingte Effekte zurückgeführt, die mit den behandelten numerischen Modellen nicht abgebildet werden.

In Bild 7.8 sind als nächstes die Maximalwerte der Durchbiegungen dargestellt, die numerisch berechnet bzw. an der Erfttalbrücke gemessen wurden. Hier zeigt sich, dass Modell 2 eine recht genaue, größtenteils auf der sicheren Seite liegende Prognose der Brückenverformungen erlaubt. Demgegenüber liefert das Koppelmodell (Modell 3) zwar teilweise eine noch genauere Einschätzung der Durchbiegungen, jedoch markiert die zugehörige Kurve eher die untere Grenze des gemessenen Durchbiegungsspektrums. Mit Modell 1 wird das Schwingungsverhalten der Erfttalbrücke erneut viel zu ungünstig eingeschätzt. Die berechneten Verformungen übersteigen die tatsächlichen Werte teilweise um das vierfache.

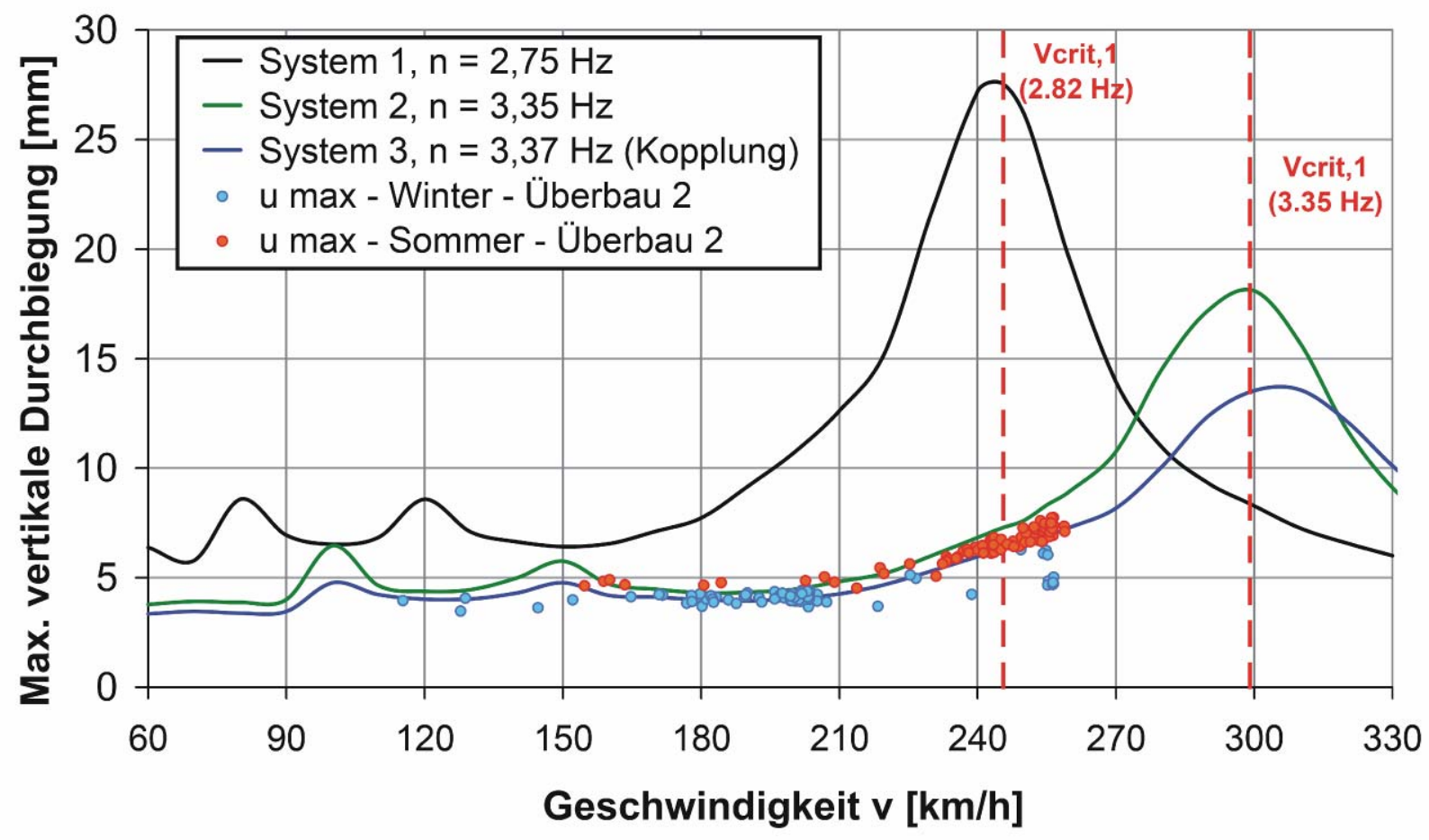

Bild 7.8: Gegenüberstellung von berechneten und gemessenen maximalen Verformungen in Feldmitte (aufgetragen jeweils in Abhängigkeit von der Überfahrtgeschwindigkeit) 
Der durchgeführte Vergleich der numerisch ermittelten mit den gemessenen Bauwerksreaktionen zeigt, dass die in Abschnitt 6 angeführten Bemessungsvorschläge im Falle der Erfttalbrücke zu einer wirklichkeitsnahen Einschätzung des dynamischen Verhaltens unter ICE-3-Zuglasten führen.

Das nach derzeitigem Stand der Technik erstellte numerische Modell 1 liefert sowohl in Bezug auf Beschleunigungen als auch in Bezug auf Durchbiegungen eine viel zu ungünstige Einschätzung des realen Systemverhaltens. Zwar liegen die Ergebnissen auf der sicheren Seite, jedoch sind eine wirklichkeitsnahe Einschätzung des dynamischen Verhaltens und damit auch eine bauwerksgerechte und wirtschaftliche dynamische Bemessung mit diesem numerischen Modell unmöglich.

Durch Modifikation der Querschnittssteifigkeit unter Verwendung des in Bild 6.1 gegebenen Korrekturbeiwertes wurde mit Modell 2 ein numerisches Modell erstellt, das die Grundlage für eine realistische dynamische Bemessung bietet. Unter Verwendung von Modell 3 lassen sich insbesondere in Bezug auf die Beschleunigungen noch genauere Ergebnisse ableiten. Jedoch muss hier angemerkt werden, dass mit diesem Modell nicht ausschließlich auf der sicheren Seite liegende Bauwerksreaktionen berechnet werden.

Abschließend wird die Momentenbeanspruchung des WIB-Überbaus untersucht. Die Bemessung der Erfttalbrücke wurde im Jahre 1999 durchgeführt [7]. Detaillierte dynamische Berechnungen zur Resonanzproblematik waren damals noch nicht Stand der Technik. Stattdessen wurden Verkehrslasten durch den Ansatz statischer Lastmodelle berücksichtigt, die mit Schwingfaktoren zu multiplizieren waren. Im konkreten Fall ergab sich unter Berücksichtigung eines dynamischen Beiwertes von $f=1,13$ für das Lastbild UIC 71 mit gleichmäßiger Verteilung der Einzellasten ein statisches Ersatzmoment von

$$
\Phi \cdot M_{\text {UIC }}=1,13 \cdot 8670=9800 \mathrm{kNm}
$$

für das die Brücke ausgelegt wurde.

Wie Bild 7.9 zeigt, wird bei Verwendung von Modell 1 dieses Moment bereits bei einer Geschwindigkeit von $225 \mathrm{~km} / \mathrm{h}$ überschritten. Geschwindigkeiten bis zu $v=250 \mathrm{~km} / \mathrm{h}$ wären auf Grundlage von Modell 1 also nicht zulässig. Dafür hätte der Überbauquerschnitt für eine charakteristische Einwirkung aus Verkehr von etwa $14000 \mathrm{kNm}$ ausgelegt werden müssen. Eine entsprechende Tragfähigkeit lässt sich mit Hilfe der WIB-Bauweise kaum erzielen.

Wird der dynamischen Bemessung hingegen das realistischere Modell 2 zugrunde gelegt, so ist das statische Ersatzmoment bis zu einer Geschwindig- 
keit von etwa $280 \mathrm{~km} / \mathrm{h}$ maßgebend. Auf Grundlage von Modell 2 sind somit Geschwindigkeiten bis zu v $=280 \mathrm{~km} / \mathrm{h}$ zulässig. Gleiches gilt für Modell 3 .

Anhand des Bemessungsbeispiels konnte gezeigt werden, wie sich die Verwendung des Korrekturfaktors zur Modifikation der Überbausteifigkeit im Rahmen der dynamischen Bemessung auswirkt. Es lassen sich damit bei der Untersuchung von Bestandsbauten deutlich höhere zulässige Geschwindigkeiten nachweisen. Damit wird die Modernisierung des Streckennetzes erleichtert und dessen Leistungsfähigkeit gestärkt.

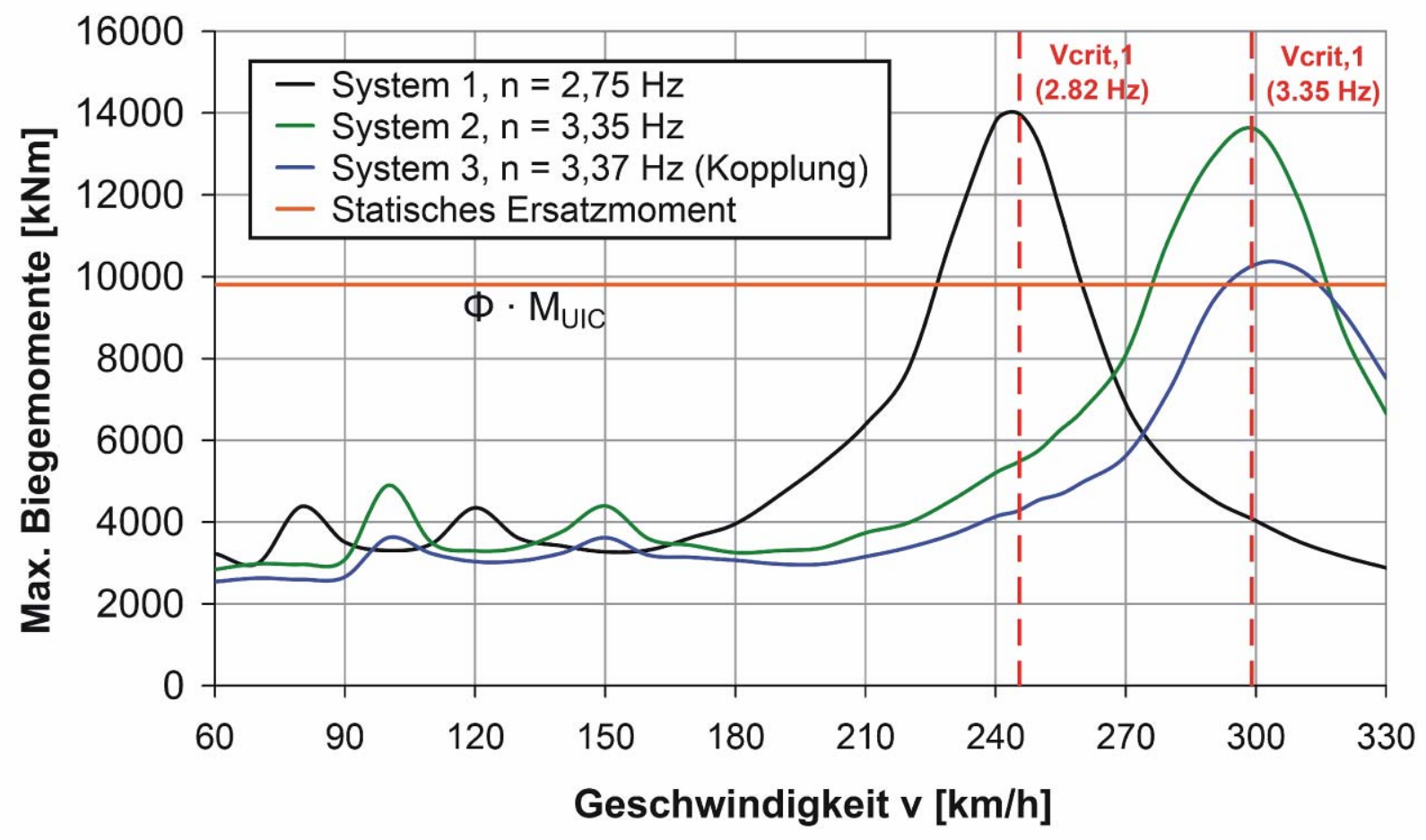

Bild 7.9: Gegenüberstellung von berechneten maximalen Biegemomenten (aufgetragen in Abhängigkeit von der Überfahrtgeschwindigkeit) und dem Bemessungsmoment aus statischen Ersatzlasten 


\section{Zusammenfassung und Ausblick}

Die dynamische Bemessung von Eisenbahnbrücken für den Hochgeschwindigkeitsverkehr erfordert die Untersuchung von Resonanzeffekten, die bei hohen Überfahrtgeschwindigkeiten von Zügen mit regelmäßigen Achsabständen bemessungsrelevant werden können. Voraussetzung für eine zutreffende Vorhersage des dynamischen Systemverhaltens ist dabei die Kenntnis der wesentlichen dynamischen Eigenschaften des untersuchten Brückenbauwerks.

Allerdings ist bei WIB-Eisenbahnbrücken gerade die rechnerische Vorhersage der resonanzbestimmenden Systemeigenfrequenzen schwierig. Im Rahmen der Arbeit ausgewertete Messungen an WIB-Bauwerken belegen eine auffällige Diskrepanz zwischen tatsächlichen Eigenfrequenzen und rechnerischen Werten, die nach den derzeit üblichen Methoden der Praxis ermittelt wurden. Sie zeigen weiterhin deutliche Unterschiede zwischen tatsächlich vorhandenen Dämpfungswerten und den normativen Vorgaben. Diese Fehleinschätzungen werden auf den Einfluss baulicher Randbedingungen zurückgeführt, der in der derzeitigen Bemessungspraxis bisher keine Berücksichtigung findet.

Das Ziel der Arbeit war eine verbesserte Vorhersage des dynamischen Systemverhaltens von WIB-Brücken kurzer bis mittlerer Spannweiten. Ausgehend von den Ergebnissen der Bauwerksmessungen sollte mit Hilfe numerischer Berechnungen untersucht werden, wie sich die konstruktive Ausbildung des tragenden Überbaus, der Lagerung sowie der in der Regel als nicht tragend angesehenen Oberbauelemente auf das dynamische Verhalten von WIB-Brücken auswirkt. Dabei sollten Modelle entwickelt werden, die eine wirklichkeitsnahe Abbildung konstruktiver Effekte zulassen und bei der Simulation von Zugüberfahrten im Hochgeschwindigkeitsbereich verwendet werden können. Schließlich sollte eine praxistaugliche Bemessungshilfe erarbeitet werden, die eine möglichst genaue Berechnung der Eigenfrequenzen von WIB-Brücken erlaubt und somit die Grundlage für eine zutreffende dynamische Bemessung schafft.

Im Rahmen der dynamischen FE-Berechnungen wurde festgestellt, dass sich insbesondere Steifigkeitsbeiträge von Randkappen und Schutzschicht erheblich auf die Brückendynamik auswirken. Dieser Effekt tritt verstärkt bei Brücken mit kleinen Stützweiten auf. Werden Kappen und Schutzschicht bei der Steifigkeitsermittlung rechnerisch berücksichtigt, ergeben sich Eigenfrequenzen und maximale Beanspruchungen bei Überfahrtsimulationen, die eine deutliche Annäherung an die realen Verhältnisse darstellen. 
Daneben hat bei mehrgleisigen Überführungsbauwerken, die aus eingleisigen WIB-Überbauten bestehen und über durchgängige Schotterschichten gekoppelt sind, die Schotterkopplung einen signifikanten Einfluss auf das dynamische Systemverhalten. Der Koppeleffekt führt dazu, dass während der Beanspruchung durch Zugverkehr neben den belasteten auch die unbelasteten Brückenträger dynamisch angeregt werden. Um diesen Effekt mittels eines geeigneten mechanischen Modells numerisch abbilden zu können, wurden experimentelle Untersuchungen an Eisenbahnschotter durchgeführt. Dabei wurden zwei nebeneinander liegende, mit Schotteraufbau versehene Biegeträger sowohl quasistatischen als auch dynamischen Lastsituationen ausgesetzt, um die Steifigkeits- und Dämpfungseigenschaften des Systems zu bestimmen. Die experimentellen Ergebnisse lieferten die Grundlage für die Implementierung von $\mathrm{Fe}-$ derelementen im numerischen Modell, mit denen die Lastübertragung durch Schotterschichten wirklichkeitsnah erfasst werden kann.

Als wesentliches Resultat der anschließenden numerischen Untersuchungen in Form von dynamischen FE-Berechungen wurde festgestellt, dass die steifigkeitsbeeinflussende Wirkung der unterschiedlichen baulichen Randbedingungen prinzipiell ähnlich abgebildet werden kann, indem die globale Biegesteifigkeit des Brückenquerschnitts ersatzweise vergrößert wird. Davon ausgehend wurde ein Bemessungsdiagramm erarbeitet, mit dessen Hilfe sich eine im Rahmen der dynamischen Bemessung anzusetzende effektive Biegesteifigkeit ermitteln lässt. Dabei wurde ein Konzept mit Korrekturbeiwerten verfolgt, das mit der derzeitigen Bemessungspraxis vereinbar ist und in einfacher Weise in die aktuelle Normung integriert werden kann.

Abschließend wurde die Praxistauglichkeit und Zuverlässigkeit des vorgeschlagenen Verfahrens anhand eines Berechnungsbeispiels vorgeführt. Zu diesem Zweck wurde eine rund $25 \mathrm{~m}$ spannende Eisenbahnbrücke, die aus zwei eingleisigen durch eine Schotterschicht gekoppelten WIB-Überbauten besteht, dynamisch untersucht. Das Beispiel zeigt, dass sich unter Verwendung der erarbeiteten Bemessungshilfe eine zuverlässigere Prognose des dynamischen Systemverhaltens von WIB-Überbauten ableiten lässt.

Nicht nur bei WIB-Brücken, sondern auch bei anderen Brückentypen wurden Diskrepanzen zwischen rechnerischen und gemessenen dynamischen Systemparametern festgestellt, siehe z.B. [33] [43] [57] [61]. Auch in diesen Fällen ließe sich das in dieser Arbeit vorgeschlagene Verfahren anwenden, um effektive Biegesteifigkeiten näherungsweise zu bestimmen. Allerdings müssten die anzusetzenden Korrekturfaktoren durch eine ausreichende Anzahl von Messuntersuchungen belegt werden. Eine Herleitung der Korrekturwerte auf rein theoreti- 
scher Basis ist nach Meinung des Verfassers noch nicht hinreichend abgesichert. Wesentliche Voraussetzung hierfür wären Messkampagnen an entsprechenden Brückenbauwerken während der Bauphase. Indem jeweils nach Fertigstellung der einzelnen Brückenteile die dynamischen Systemparameter messtechnisch ermittelt würden, ließen sich den einzelnen baulichen Elementen eindeutig Steifigkeitsanteile zuordnen. Die effektive Gesamtsteifigkeit des Querschnitts könnte dann als Summe der Einzelsteifigkeiten theoretisch abgeleitet werden. 


\section{Literaturverzeichnis}

[1] Baeßler, M.: Lageveränderungen des Schottergleises durch zyklische und dynamische Beanspruchungen. Dissertation an der Technischen Universität Berlin, 2008

[2] Bagayoko, L.; Koch, E.; Patz, R.: Dynamik von Eisenbahnbrücken, StahlbauKalender 2008, Ernst \& Sohn, Berlin, 2008

[3] Bathe, K. J. \& Wilson, E. L.: Numerical methods in finite element analysis. Prentice-Hall, Englewood Cliffs, New Jersey, 1976

[4] Bungard, V.; Mahowald, J.; Waldmann, D.; Maas, S.; Zürbes, A.: Dynamische Zustandsbewertung einer Verbundbrücke - Beobachtung äußerer und nichtlinearer Einflüsse auf die modalen Eigenschaften, Beton- und Stahlbetonbau (2010), H.8, S. $509-520$

[5] Clough, R. W.; Penzien, J.: Dynamics of Structures. McGraw-Hill Kogakusha, Tokyo 1975

[6] DB Netz AG, Unveröffentlichte Berichte zu Messkampagnen an diversen WIBEisenbahnbrücken auf deutschen HGV-Strecken, 2000-2003

[7] DB ProjektBau GmbH: Unveröffentlichter Standsicherheitsnachweis für das Bauvorhaben EÜ Erfttalstraße, ABS 4 Köln - Aachen, km 21,223, 1999

[8] DB-Richtlinie 804: Eisenbahnbrücken (und sonstige Ingenieurbauwerke) planen, bauen und instand halten, DB Netz AG, 2003

[9] DIN EN 1337-3: Lager im Bauwesen, Teil 3 Elastomerlager, Deutsche Fassung EN 1337-3:2005, Ausgabe 2005

[10] DIN EN 1991-2: Einwirkungen auf Tragwerke, Teil 2: Verkehrslasten auf Brücken, Deutsche Fassung EN 1991-2:2003, Ausgabe 2004

[11] DIN-Fachbericht 101: Einwirkungen auf Brücken, Ausgabe 2009

[12] DIN-Fachbericht 104: Verbundbrücken, Ausgabe 2009

[13] Dinkler, D.; Löhr, M.: Resonanzgefährdung von Eisenbahnbrücken bei Hochgeschwindigkeitsüberfahrten. In Möller, B. (Hrsg.): Berichte der Fachtagung Baustatik-Baupraxis 9, Institut für Statik und Dynamik der Tragwerke, TU Dresden, S. 347-356, 2005

[14] Eisenmann, J.; Kaess, G.: Das Verhalten des Schotters unter Belastung. Eisenbahntechnische Rundschau, 29 (1980), H. 3, S. 201-208

[15] Eisenmann, J.; Rump, R.: Ein Schotteroberbau für hohe Geschwindigkeiten. Eisenbahntechnische Rundschau, 46 (1997), H. 3, S. 99-108

[16] ERRI (European Railway Research Institute) D 214 (Eisenbahnbrücken für Geschwindigkeiten über $200 \mathrm{~km} / \mathrm{h}$ ), Report 4 (Wechselwirkung zwischen Zug und Brücke) und Report 9 (Abschlussbericht), 1999 
[17] Fink, J.; Mähr, T.: Vergleich und Beurteilung unterschiedlicher Lastmodelle für die Ermittlung der dynamischen Antwort von Zugüberfahrten über Brücken bei Hochgeschwindigkeitsverkehr, Stahlbau 78 (2009), H.10, S.733-741

[18] Flesch, R.; Handel, C.: Schwingungsmessungen an 3 Eisenbahnbrücken bei Hochgeschwindigkeitsfahrten, VDI-Berichte Nr. 1941, 2006

[19] FOX - Auswerteroutinen Monitoring Erfttalbrücke, Programmversion 1.40, Weil, F.; Rauert, T., Lehrstuhl für Stahlbau der RWTH Aachen, 2010

[20] Frýba, L.: Dynamics of Railway Bridges, 2nd. Ed. Thomas Telford, London, 1996

[21] Geier, R.; Österreicher, M.; Pircher, M.: Langzeitmessungen an einer Eisenbahnbrücke. Beton- und Stahlbetonbau 103 (2008), H. 6, S. 378-387

[22] Guldenfels, R.: Die Alterung von Bahnschotter aus bodenmechanischer Sicht. Veröffentlichungen des Instituts für Geotechnik (IGT) der ETH Zürich, 209. vdf Hochschulverlag, 1996

[23] Handel, C.: Richtlinie für dynamische Messungen an Eisenbahnbrücken, Tagungsbeitrag D-A-CH Tagung, Wien, 2007

[24] Haupt, W. (Hrsg.): Bodendynamik - Grundlagen und Anwendung, Friedr. Vieweg \& Sohn Verlagsgesellschaft mbH, Braunschweig 1998

[25] Hauser, A.; Adam, C.: Abschätzung der Schwingungsantwort von Brückentragwerken für Hochgeschwindigkeitszüge, Tagungsbeitrag D-A-CH Tagung, Wien, 2007

[26] Hughes, T. J. R.: The Finite Element Method - Linear Static and Dynamic Finite Element Analysis, Dover Publications, Mineola, New York, 2000

[27] InfoCAD Programmversion 10.30b, InfoGraph GmbH, www.infocad.de, 2010

[28] Knothe, K.: Gleisdynamik, Ernst \& Sohn Verlag, 2001

[29] Kruse, H.: Modellgestützte Untersuchung der Gleisdynamik und des Verhaltens von Eisenbahnschotter, Fortschritt-Berichte VDI Reihe 12 Nr. 508, VDI Verlag Düsseldorf, 2002

[30] Mähr, T.: Theoretische und experimentelle Untersuchungen zum dynamischen Verhalten von Eisenbahnbrücken mit Schotteroberbau unter Verkehrslast, Dissertation an der Technischen Universität Wien, 2009

[31] Mähr, T.; Fink, J.: Modal Testing and Analysis of steel railway bridges - Influence of the ballast superstructure on the dynamic properties of a full-scale bridge model, Proceedings of the Eurosteel Conference 2008

[32] Martinez-Rodrigo, M. D. ; Domenech, A.; Museros, P.: Maximum resonance and cancellation phenomena in elastically-supported beams and its application to railway bridges under high-speed traffic, Proceedings of ISMA Conference (International Conference on Noise and Vibration), Leuven, Belgium, 2010 
[33] Marx, S.; Geißler, K.: Erfahrungen zur Modellierung und Bewertung von Eisenbahnbrücken mit Resonanzrisiko, Stahlbau 79 (2010), H. 3, S. 188-198

[34] MATLAB Programmversion R2010b, MathWorks, 2010

[35] Meskouris, K.: Baudynamik - Modelle, Methoden, Praxisbeispiele, Ernst \& Sohn Verlag, Berlin, 1999

[36] Museros, P.; Alarcon, E.: Influence of the second bending mode on the response of high-speed bridges at resonance. Journal of Structural Engineering ASCE, March 2005, p. 405-415

[37] Museros, P.; Martinez-Rodrigo, M. D.: The cancellation phenomenon on simply supported beams and plates subjected to moving loads, Proceedings of the $12^{\text {th }}$ International Conference on civil structures and environmental engineering computing, Madeira, Portugal, 2010

[38] Natke, H. G.: Baudynamik - Einführung in die Dynamik mit Anwendungen aus dem Bauwesen. Stuttgart: B.G. Teubner, 1989

[39] ÖBB Infrastruktur: B 45 - Technische Richtlinie für Eisenbahnbrücken und sonstige konstruktive Ingenieurbauwerke, Februar 2011

[40] Petersen, C.: Dynamik der Baukonstruktionen, Friedr. Vieweg \& Sohn Verlagsgesellschaft mbH, Braunschweig / Wiesbaden, 2000

[41] Pfeifer, R.; Mölter, T.: Handbuch Eisenbahnbrücken - Grundsätze für Planung und Konstruktion sowie Hinweise auf Bauverfahren, DVV Media Group GmbH, 2008

[42] Pietruszka, W. D.: Matlab und Simulink in der Ingenieurpraxis - Modellbildung, Berechnung und Simulation. B. G. Teubner Verlag, Wiesbaden 2006

[43] Pircher, H.; Stadler, C.; Glatzl, J.; Seitz, P.: Dynamische Berechnung von Eisenbahnbrücken im Zuge von Hochgeschwindigkeitsstrecken - Ein alternatives Verfahren zur Simulation von Zugüberfahrten, Bautechnik 86 (2009), H.1, S.1-13

[44] Ramondenc, P.: Vom Einfluss hoher Geschwindigkeiten auf den Entwurf von Eisenbahnbrücken am Beispiel der Stahlbrücken auf der Hochgeschwindigkeitsstrecke des TGV Mediterrannee, Stahlbau 67 (1998), H. 8, S. 652-658

[45] Rauert, T.; Bigelow, H.; Hoffmeister, B.; Feldmann, M.: On the prediction of the interaction effect caused by continuous ballast on filler beam railway bridges by experimentally supported numerical studies, Engineering Structures 32 (December 2010), p. 3981-3988

[46] Rauert, T.; Bigelow, H.; Hoffmeister, B.; Feldmann, M.; Patz, R.; Lippert, P.: Zum Einfluss baulicher Randbedingungen auf das dynamische Verhalten von WIBEisenbahnbrücken -Teil 1: Einführung und Messuntersuchungen an WIB-Brücken, Bautechnik (2010), H.11, S. 665-672

[47] Rauert, T.; Bigelow, H.; Hoffmeister, B.; Feldmann, M.; Patz, R.; Lippert, P.: Zum Einfluss baulicher Randbedingungen auf das dynamische Verhalten von WIB- 
Eisenbahnbrücken -Teil 2: Numerische Untersuchungen und Bemessungsvorschläge, Bautechnik (2010), H.12, S. 751-760

[48] Rauert, T.; Feldmann, M.; He, L.; De Roeck, G.: Calculation of bridge deformations due to train passages by the use of strain and acceleration measurements from bridge monitoring, Proceedings of EURODYN'11 Conference (International Conference on Structural Dynamics), Leuven, Belgium, 2011

[49] Rauert, T.; Hoffmeister, B.: Long-term Monitoring of a Twin Composite Filler Beam Railway Bridge with Continuous Ballast, Proceedings of the ISEV'09 Conference (International Symposium on Environmental Vibrations), Beijing, China, 2009

[50] Rauert, T.; Hoffmeister, B.; Cantieni, R.; Brehm, M.; Zabel, V.: Experimental modal analysis of a twin composite filler beam railway bridge for high-speed trains with continuous ballast, Proceedings of the IABMAS`08 Conference (International Association for Bridge Maintenance and Safety), Seoul, Korea, 2008

[51] Rebelo, C.; Simoes da Silva, L. Rigueiro, C, Pircher, M.: Dynamic behaviour of twin single-span ballasted railway viaducts - Field measurements and modal identification, Engineering Structures 30 (2008) 2460-2469

[52] RFCS-project DETAILS: Design for optimal life cycle costs (LCC) of high-speed railway bridges by enhanced monitoring systems, RFSR-CT-2006-00032, Laufzeit 2006-2009, Abschlussbericht in Bearbeitung

[53] Rigueiro, C.; Rebelo, C.; Simoes da Silva, L.: Influence of ballast models in the dynamic response of railway viaducts, Journal of Sound and Vibration 329 (2010) 3030-3040

[54] Schmitt, V.; Seidl, G.: Eisenbahnbrücken in Stahlverbundbauweise, Stahlbau 79 (2010), H.3, S. 159-166

[55] Schmitt, V.; Seidl, G.; Vogel, C.; Schülke, B.; Koch, E: Untersuchungen zum verstärkten Einsatz von Stahlverbundkonstruktionen bei Brücken kleiner und mittlerer Stützweiten, Forschungsvorhaben P 629, Verlag und Vertriebsgesellschaft mbH, Düsseldorf, 2005

[56] Simoes, R.; Calcada, R.; Delgado, R.: Track-bridge interaction in railway lines: Numerical modelling and application, Workshop on track-bridge interaction on high-speed railways, FEUP Porto, 2007

[57] Spengler, M.; Duda, H.; Graubner, C. A.: Beanspruchung von Eisenbahnbrücken durch Hochgeschwindigkeitsverkehr - Einfluss der Oberbauart „Feste Fahrbahn“ auf die strukturdynamischen Eigenschaften und die dynamische Tragwerksreaktion, VDI-Berichte Nr. 1941, 2006

[58] Spengler, M.; Graubner, C. A.: Vorschlag für ein Antwortspektrum zur Erfassung der Dynamik von Eisenbahnbrücken, Bauingenieur Band 84, S. 1-9, Januar 2009

[59] Stadler, C.: „RL Dynamik“ Richtlinie für die dynamische Berechnung von Eisen- 
bahnbrücken, Tagungsbeitrag D-A-CH Tagung, Wien, 2007.

[60] Wenzel, H.: Health monitoring of bridges. John Wiley \& Sons, Ltd, 2009

[61] Winselmann D.; Fahlbusch D.: Überprüfung der Resonanzgefahr bei bestehenden und neuen Eisenbahnbrücken, VDI-Berichte Nr. 1941, 2006

[62] Wißbrock, H.: Untersuchungen zur Fugendämpfung zusammengesetzter Bauteile. Fortschr.-Ber. VDI Reihe 11 Nr. 68. Düsseldorf: VDI Verlag 1985

[63] WSTAB Programmversion 1.0, Heinemeyer, C., Lehrstuhl für Stahlbau der RWTH Aachen, 2010

[64] Xia, H.; Zhang, N.; Guo, W.W.: Analysis of resonance mechanism and conditions of train-bridge system. Journal of Sound and Vibration, 297 (2006), p. 810-822

[65] Yang, Y.-B.; Yau, J.-D. ; Hsu, L.-C. : Vibration of simple beams due to trains moving at high speeds, Engineering Structures Vol. 19, No. 11, p. 936-944, 1997

[66] Zabel, V., Brehm, M.: Das dynamische Verhalten von Eisenbahnbrücken mit kurzer Spannweite - numerische und experimentelle Untersuchungen, Bauingenieur Band 83 (D-A-CH-Mitteilungsblatt), S. 9-14, März 2008

[67] Zabel, V.; Brehm, M.; Bucher, C.: Seasonal changes of the dynamics of railway bridges with steel girders embedded in concrete, Proceedings of the EVACES'07 Conference (Experimental Vibration Analysis for Civil Engineering Structures), Porto, Portugal, 2007

[68] Zacher, M.: Dynamisches Verhalten von Eisenbahnschotter im Fugenbereich von Brücken. Dissertation an der Technischen Universität Berlin, 2005

[69] Zacher, M.: Dynamik von Eisenbahnbrücken und dessen Auswirkungen auf den Oberbau. Eisenbahningenieur, 52 (2001), H. 2, S. 41-45

[70] Zacher, M.; Baeßler, M.: Dynamic behaviour of ballast on railway bridges. Tagungsband: Advanced course - Dynamics of high-speed railway bridges, pages 125-142, Porto 2005 